\title{
Zerstörungsfreie Wurzelortung mit geophysikalischen Methoden im urbanen Raum
}

\author{
Dissertation \\ zur Erlangung des mathematisch-naturwissenschaftlichen Doktorgrades \\ „Doctor rerum naturalium“ \\ der Georg-August-Universität Göttingen \\ im Promotionsprogramm Geographie \\ der Georg-August University School of Science (GAUSS)
}

vorgelegt von

Mitja Johannes Vianden

aus Hamburg

Göttingen 2013 
Betreuungsausschuss:

Prof. Dr. Gerhard Gerold (Abteilung Landschaftsökologie, Geographisches Institut, Universität Göttingen)

Prof. Dr. Steffen Rust (Fakultät Ressourcenmanagement, HAWK Göttingen)

Mitglieder der Prüfungskommission:

Referent: Prof. Dr. Gerhard Gerold (Abteilung Landschaftsökologie, Geographisches Institut, Universität Göttingen)

Korreferent: Prof. Dr. Steffen Rust (Fakultät Ressourcenmanagement, HAWK Göttingen)

weitere Mitglieder der Prüfungskommission:

Prof. Dr. Andreas Junge (Abteilung Angewandte Geophysik, Institut für Geowissenschaften Facheinheit Geophysik, Universität Frankfurt)

Prof. Dr. Martin Kappas (Abteilung Kartographie, GIS und Fernerkundung, Geographisches Institut, Universität Göttingen)

Prof. Dr. Yakov Kuzyakov (Fakultät für Forstwissenschaften und Waldökologie, Abteilung Ökopedologie der gemäßigten Zonen, Universität Göttingen)

Prof. Dr. Jürgen Schmidt (Fachgebiet Boden- und Gewässerschutz, Institut für Bohrtechnik und Fluidbergbau, Universität Freiberg)

Tag der mündlichen Prüfung: 25.07.2013 


\section{Danksagung}

Mein besonderer Dank gilt Herrn Prof. Dr. Steffen Rust, der die Anregung zu dieser Arbeit gegeben hat. Er hat das Dissertationsprojekt betreut und mit zahlreichen Vorschlägen und Anmerkungen unterstützt. Für die Betreuung der Arbeit danke ich ebenso meinem Doktorvater, Herrn Prof. Dr. Gerhard Gerold, der mich unkompliziert in die Abteilung Landschaftsökologie des Geographischen Instituts aufgenommen hat.

Weiterer Dank gilt Herrn Prof. Dr. Andreas Junge, Herrn Prof. Dr. Martin Kappas, Herrn Prof. Dr. Yakov Kuzyakov und Herrn Prof. Dr. Jürgen Schmidt für die Mitarbeit in der Prüfungskommission.

Für die Möglichkeit, meine Arbeitsergebnisse zu diskutieren und die damit verbundenen Anregungen für die vorliegende Dissertation, bedanke ich mich in diesem Zusammenhang sowohl beim Team des Projekts „Städtischer Wurzelraum“ an der HAWK als auch beim Team der Abteilung Landschaftsökologie des Geographischen Instituts.

Die Durchführung der Arbeiten im Rahmen des angewandten Forschungsschwerpunkts „Städtischer Wurzelraum“ wurden durch Mittel aus dem „Niedersächsischen Vorab“ der Volkswagenstiftung ermöglicht. Für deren großzügige Bereitstellung bedanke ich mich bei der Arbeitsgemeinschaft für innovative Projekte (AGiP) in Hannover.

Für die freundliche Aufnahme und die unkomplizierte Zusammenarbeit bedanke ich mich bei allen Mitarbeitern der Fakultät Ressourcenmanagement der HAWK.

Besonderer Dank gilt Herrn Dr. Falko Kuhnke für die fortwährende, geduldige Erläuterung und Diskussion von geophysikalischen Fragestellungen und Zusammenhängen. Weiterhin danke ich ihm für die Entwicklung und Instandsetzung von Messgeräten sowie für die Anregungen und die Unterstützung bei der Planung und Durchführung von Feldarbeiten.

Bei Herrn Prof. Dr. Ulrich Weihs möchte ich mich insbesondere für die erfolgreiche Zusammenarbeit bei den Messungen am Berliner Landwehrkanal bedanken. In diesem Zusammenhang danke ich auch Frau Dr. Annette Ernst und Herrn Björn Röske vom WSA Berlin sowie Frau Ursula Kleimeier von der Bürgerinitiative "Bäume am Landwehrkanal".

Für die Bereitstellung von Messflächen bedanke ich mich bei Frau Busse und Herrn Strümper vom Grünflächenamt der Stadt Göttingen, Herrn Mattner vom Grünflächendienst der Universität Göttingen, Herrn Callauch vom Neuen Botanischen Garten sowie Herrn Meng vom Forstbotanischen Garten der Universität Göttingen. Besonderer Dank gilt Herrn Jan Willem de Groot für die Zusammenarbeit bei den Messungen und Wurzelfreilegungen in Nimwegen, sowie Herrn Olaf Florin bei der Zusammenarbeit in Fulda.

Für die tatkräftige Unterstützung bei zahlreichen Messungen bedanke ich mich bei Stefan Pfeiffer. Außerdem danke ich Anja Lücke, Marco Pollmeier, Karsten Weller, Marco Gronwald, David Endter, Michel Nünnerich und Martin Reuter für ihre Mithilfe bei Messungen.

Für die geduldige Einarbeitung in das Radarmesssystem, die Ausleihe der 900 MHz-Antenne, 
zahlreichen Ratschlägen für die Durchführung und Interpretation von GPR-Messungen sowie die kritische Durchsicht der Arbeit danke ich Frau Dr. Susanne Kathage. Bei Herrn Dr. Dean Goodman bedanke ich mich für die Bearbeitung der Schwarzkieferndaten mit gpr-slice.

Ganz besonderer Dank gilt meiner Familie, die mich immer unterstützt hat und immer davon überzeugt war, dass ich diese Arbeit erfolgreich abschließen werde. Meinem Bruder Kolja danke ich darüber hinaus für die gewissenhafte Durchsicht meines Manuskripts. Ebenfalls von ganzem Herzen danke ich Vanessa Behling, die mich insbesondere in der Endphase der Arbeit immer wieder davon überzeugt hat, dass ich auf dem richtigen Weg bin. 


\section{Inhaltsverzeichnis}

Danksagung

Abbildungsverzeichnis v v

Tabellenverzeichnis vii

\begin{tabular}{|ll}
\hline Abkürzungsverzeichnis & ix
\end{tabular}

\begin{tabular}{lll}
\hline 1 & Einleitung & 1
\end{tabular}

2 Stand des Wissens 3

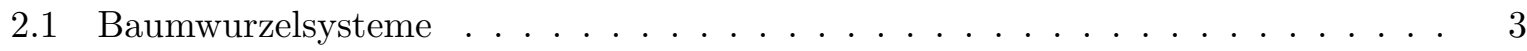

$2.1 .1 \quad$ Einteilung und Aufbau . . . . . . . . . . . . . . . . . . 3

$2.1 .2 \quad$ Wurzelsysteme in Abhängigkeit von Baumart und Standort . . . . . . . . 5

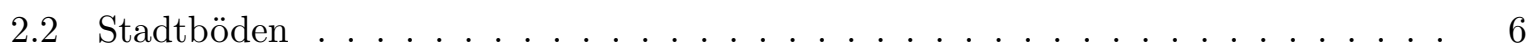

2.3 Geophysikalische Untersuchungsverfahren $\ldots \ldots \ldots \ldots$. . . . . . . . . . 7

2.3.1 Grundlagen elektromagnetischer Methoden . . . . . . . . . . . . . 7

2.3 .2 Georadar . . . . . . . . . . . . . . . . . . . . 8

$2.3 .3 \quad$ Geoelektrik . . . . . . . . . . . . . . . . . . . . . . . . . . . . . 19

2.4 Elektromagnetische Eigenschaften von Böden und Wurzeln . . . . . . . . . . . . 23

$2.4 .1 \quad$ Dielektrische Permittivität $\varepsilon_{\mathrm{r}} \ldots \ldots \ldots \ldots \ldots \ldots \ldots$

2.4 .2 Elektrische Leitfähigkeit $\sigma \ldots \ldots \ldots \ldots$. . . . . . . . . . . . 23

2.5 Vorarbeiten zur Wurzeldetektion mit geophysikalischen Methoden . . . . . . . . 24

2.5 .1 Wurzeldetektion mit Georadar . . . . . . . . . . . . . . 24

$2.5 .2 \quad$ Wurzeldetektion mit Geoelektrik . . . . . . . . . . . . . . . . . 25

3 Material und Methoden 29

$3.1 \quad$ Untersuchungsdesign . . . . . . . . . . . . . . . . . . . . . . . . . . . . . . . 29

$3.1 .1 \quad$ 2D-Georadarmessungen in den Versuchsbecken . . . . . . . . . . . . . . . 32

3.1 .2 3D-Messungen zu grundlegenden Fragestellungen . . . . . . . . . . . . . . 32

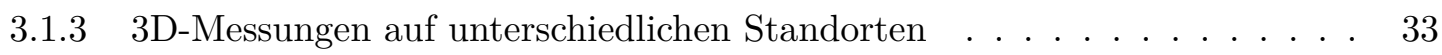

3.2 Untersuchungsstandorte $\ldots \ldots \ldots \ldots \ldots \ldots \ldots$

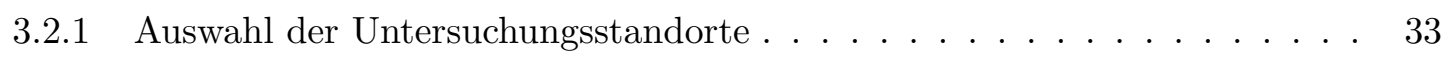

3.2 .2 Versuchsbecken . . . . . . . . . . . . . . . . . . . . 36

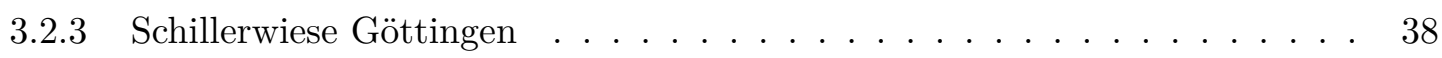

3.2 .4 Landwehrkanal Berlin . . . . . . . . . . . . . . . . . . . . . . 38

3.2 .5 Weitere Messflächen . . . . . . . . . . . . . . . . . . . 40 
3.3 Georadarmessungen $\ldots \ldots \ldots \ldots$. . . . . . . . . . . . . . . . . . 40

$3.3 .1 \quad$ Messsystem . . . . . . . . . . . . . . . . . . . . . . . . . . 40

3.3 .2 Durchführung der Messungen . . . . . . . . . . . . . . . . . . . . . . 41

$3.3 .3 \quad$ Datenbearbeitung, Ergebnisdarstellung und Auswertung . . . . . . . . . . 43

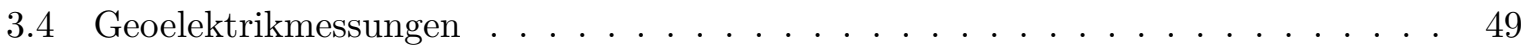

$3.4 .1 \quad$ Messsystem $\ldots \ldots \ldots \ldots$. . . . . . . . . . . . . . . . . . . . . . . . . . . . 49

3.4 .2 Durchführung der Messungen . . . . . . . . . . . . . . . . . . . . . . . . 49

$3.4 .3 \quad$ Datenbearbeitung, Ergebnisdarstellung und Auswertung . . . . . . . . . . 50

$3.5 \quad$ Wurzelfreilegungen und Beprobungen . . . . . . . . . . . . . . . . 51

3.6 Statistische Analysen . . . . . . . . . . . . . . . . . . . . . . . . . . . 53

$\begin{array}{lll}4 & \text { Ergebnisse } & 55\end{array}$

4.1 2D-Georadarmessungen in den Versuchsbecken . . . . . . . . . . . . . . 55

$4.1 .1 \quad$ 2D-Radargramme der einzelnen Versuchsbecken . . . . . . . . . . . . . 55

$4.1 .2 \quad$ Ausgewählte wavelets aus den Versuchsbecken. . . . . . . . . . . . . . . . 58

$4.1 .3 \quad$ Korrelation von Radarlaufzeiten und eingemessenen Distanzen . . . . . . 63

$4.2 \quad$ 3D-Georadarmessungen zu grundlegenden Fragestellungen . . . . . . . . . . . . . 65

4.2 .1 Antennenpolarisation . . . . . . . . . . . . . . . . . 65

4.2 .2 Rasterdichte . . . . . . . . . . . . . . . . . . . 71

4.2 .3 Reproduzierbarkeit . . . . . . . . . . . . . . . . . . . . . . 74

4.2 .4 Nachbearbeitung und Filter . . . . . . . . . . . . . . . . . 77

$4.2 .5 \quad$ Messfrequenz . . . . . . . . . . . . . . . . . . . . . . . . 79

4.2 .6 Verifikation der Ergebnisse $\ldots \ldots \ldots$. . . . . . . . . . . 80

4.3 3D-Georadar- und Geoelektrikmessungen auf ausgewählten Standorten . . . . . . 95

$4.3 .1 \quad$ Schillerwiese Göttingen $\ldots \ldots \ldots \ldots$. . . . . . . . . . . . . . 95

4.3 .2 Landwehrkanal Berlin . . . . . . . . . . . . . . . . . . . . . . . . . . 105

4.3 .3 Weitere Messflächen . . . . . . . . . . . . . . . . . . . . . . . 115

$\begin{array}{lll}5 & \text { Diskussion } & 123\end{array}$

$5.1 \quad$ 2D-Georadarmessungen in den Versuchsbecken $\ldots \ldots$. . . . . . . . . . . 123

5.2 3D-Georadarmessungen zu grundlegenden Fragestellungen . . . . . . . . . . . . 125

5.3 3D-Georadar- und Geoelektrikmessungen auf ausgewählten Standorten . . . . . . 127

5.4 Anwendbarkeit der Messverfahren in der Praxis . . . . . . . . . . . . . . . . 130

5.5 Ausblick und weiterer Forschungsbedarf $\ldots \ldots \ldots \ldots$. . . . . . . . 135

\begin{tabular}{lll}
\hline 6 & Zusammenfassung & 137
\end{tabular}

Literatur

A Anhang vii

A.1 Materialeigenschaften . . . . . . . . . . . . . . . . . vii

A.2 Aufbau der Versuchsbecken . . . . . . . . . . . . . . . . . ix

A.3 Lage und Fotos der Messflächen . . . . . . . . . . . . . . . . . . . . . xi 
A.4 Gesamtradargramme und -tomogramme der einzelnen Messflächen . . . . . . . . xx

Wissenschaftlicher Lebenslauf . . . . . . . . . . . . . . . . . . . xliv 



\section{Abbildungsverzeichnis}

$2.1 \quad$ Einteilung der Wurzelsysteme einheimischer Baumarten. . . . . . . . . . . . . . . 4

2.2 Einteilung von Wurzeldurchmessern in unterschiedliche Größenklassen . . . . . . 5

2.3 Messprinzip des Bodenradars. . . . . . . . . . . . . . . . . . . . . . . . . . .

$2.4 \quad$ A-, B- und C- Scans bei Bodenradarmessungen. . . . . . . . . . . . . . . . 10

2.5 Vertikale Auflösung bei Bodenradarmessungen. . . . . . . . . . . . . . . . . . . 11

$2.6 \quad$ Horizontale Auflösung bei Bodenradarmessungen. . . . . . . . . . . . . . . . . . 12

2.7 Umlaufende Welle und Zwei-Wege-Reflexion an Wurzeln. . . . . . . . . . . . . . 13

2.8 Visualisierungsmöglichkeiten für 3D-Georadardatensätze. $\ldots \ldots \ldots \ldots$. . . . . 16

$2.9 \quad$ Entstehung einer Diffraktionshyperbel bei Bodenradarmessungen. . . . . . . . . . 17

2.10 Geschwindigkeitsbestimmung mittels Hyperbelbreite. . . . . . . . . . . . . . . . 18

2.11 Verschiedene Geoelektrikanordnungen. . . . . . . . . . . . . . . . . . . . 21

2.12 Datenerfassung und Visualisierung bei Geoelektrikmessungen. . . . . . . . . . . . 22

3.1 Schematische Darstellung des Untersuchungsdesigns. . . . . . . . . . . . . . . . . 31

3.2 Anordnung der Wurzelattrappen in den Versuchsbecken. . . . . . . . . . . . . . 37

3.3 Eingesetztes Georadarsystem GSSI SIR-3000. . . . . . . . . . . . . . . . . . 41

$3.4 \quad$ Schematische Darstellung der Georadarmessungen in den Versuchsbecken. . . . . 42

$3.5 \quad$ Schematische Darstellung der 3D-Messungen an Bäumen. . . . . . . . . . . . . 43

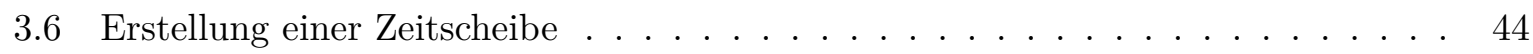

3.7 Darstellung verschiedener Laufzeitfenster als Zeitscheibe. . . . . . . . . . . . . . . . . 45

3.8 Vergleich von zwei Zeitscheiben der Messfläche Schwarzkiefer . . . . . . . . . . . 46

3.9 Auswahl der repräsentativen Zeitscheiben für die jeweilige Messfläche. . . . . . . 48

3.10 Messaufbau bei den durchgeführten Geoelektrikuntersuchungen. . . . . . . . . . . 49

3.11 Verifikationsarten . . . . . . . . . . . . . . . . . . 51

3.12 Vorgehen bei der Verifikation auf der Schillerwiese. . . . . . . . . . . . . . . . 52

$4.1 \quad$ 2D-Radargramm zu Becken 1. . . . . . . . . . . . . . . . . 56

4.2 2D-Radargramm zu Becken 2. . . . . . . . . . . . . . . . . . . . 57

4.3 2D-Radargramm zu Becken $3 . \ldots \ldots \ldots \ldots \ldots$

4.4 2D-Radargramm zu Becken $4 . \ldots \ldots \ldots \ldots \ldots \ldots$. . . . . . . . . . . . . . . . . . . . . . . . . .

4.5 Ausgewählte wavelets aus Becken $1 . \quad \ldots \ldots \ldots \ldots$. . . . . . . . 61

4.6 Ausgewählte wavelets aus Becken $2 . \quad \ldots \ldots \ldots \ldots$. . . . . . . . . 62

4.7 Korrelation von bekannten und berechneten Reflexionswegen. . . . . . . . . . . . 64

4.8 Korrelation von bekannter und berechneter Tiefe. . . . . . . . . . . . . . . . . 64

4.9 Antennenanordnung zur Messfläche (Messbeispiel 1). . . . . . . . . . . . . . 66

4.10 Antennenanordnung zur Messfläche (Messbeispiel 1 und 2). . . . . . . . . . . 67 
4.11 Antennenanordnung zur Messfläche (Messbeispiel 2). . . . . . . . . . . . . . 69

4.12 Antennenanordnung zur Messfläche (Messbeispiel 3). . . . . . . . . . . . . . . . . . 70

4.13 Antennenanordnung zur Messfläche (Messbeispiel 4). . . . . . . . . . . . . . . . . 70

4.14 Rasterabstände bei 3D-Georadarmessungen (Rohdaten). . . . . . . . . . . . . . . 72

4.15 Rasterabstände bei 3D-Georadarmessungen (bearbeitete Daten). . . . . . . . . . 73

4.16 Reproduzierbarkeitsmessung an der Schwarzkiefer. . . . . . . . . . . . . . 75

4.17 Untersuchung der Reproduzierbarkeit durch Bildkorrelation . . . . . . . . . . . 76

4.18 Bearbeitung von GPR-Datensätzen mit unterschiedlichen Filtern . . . . . . . . . 78

4.19 Einsatz verschiedener Messfrequenzen an der Schwarzkiefer. . . . . . . . . . . . . 79

4.20 Wurzelfreilegung auf der Messfläche Silberahorn Landwehrkanal. . . . . . . . . . 80

4.21 Wurzelfreilegung auf der Messfläche Hybridpappel Landwehrkanal. . . . . . . . . 81

4.22 Wurzelfreilegung auf der Messfläche Kastanie Kreuzkirche Fulda. . . . . . . . . . 83

4.23 Wurzelfreilegung auf der Messfläche Große Kastanie Landwehrkanal. . . . . . . . 85

4.24 Wurzelfreilegung auf der Messfläche Kleine Kastanie Landwehrkanal. . . . . . . . 86

4.25 Wurzelfreilegung auf der Messfläche Kastanie Archipelstraat Nimwegen. . . . . . 88

4.26 Kleinräumige Freilegungen auf der Göttinger Schillerwiese. . . . . . . . . . . . . 89

4.27 Statistische Auswertung der Beprobungen auf der Messfläche Schwarzkiefer. . . . 91

4.28 Statistische Auswertung der Beprobungen auf der Messfläche Buchengruppe. . . 92

4.29 Zusammenhang zwischen spezifischem Widerstand und Bodenwassergehalt. . . . 93

4.30 Georadarmessung an der Schwarzkiefer. . . . . . . . . . . . . . . . . 96

4.31 Geoelektrikmessung an der Schwarzkiefer. . . . . . . . . . . . . . . . . . . . 97

4.32 Vergleich von Georadar- und Geoelektrikmessung an der Fichtengruppe. . . . . . 98

4.33 Georadarmessung an der Fichte. . . . . . . . . . . . . . . . . . . . . . . . . . . . . . . 100

4.34 Geoelektrikmessung an der Fichte. . . . . . . . . . . . . . . . . . . . . . . . . . . . . . . 101

4.35 Georadarmessung an der Buchengruppe. . . . . . . . . . . . . . . . . . . 103

4.36 Vergleich von Georadar- und Geoelektrikmessung an der Buchengruppe. . . . . . 104

4.37 Vergleich von Georadar- und Geoelektrikmessung am Silberahorn. . . . . . . . 106

4.38 Vergleich von Georadar- und Geoelektrikmessung an der Hybridpappel. . . . . . 107

4.39 Georadarmessung an der Großen Kastanie. . . . . . . . . . . . . . . . . . . . . . . . . . . . 108

4.40 Geoelektrikmessung an der Großen Kastanie. . . . . . . . . . . . . . . . . . . 109

4.41 Georadarmessung an der Kleinen Kastanie. . . . . . . . . . . . . . . . . . 110

4.42 Geoelektrikmessung an der Kleinen Kastanie. . . . . . . . . . . . . . . . . . . 112

4.43 2D-Geoelektrikmessung am Corneliusufer mit langer Auslage . . . . . . . . . . 113

4.44 Foto der Messfläche Amberbaum. . . . . . . . . . . . . . . . . . . . . 115

4.45 Georadarmessung am Amberbaum. . . . . . . . . . . . . . . . . . . . . 116

4.46 Georadarmessung an der Kastanienreihe Goldschmidtstraße. . . . . . . . . . . . . 118

4.47 Georadarmessung an der Kastanie Archipelstraat Nimwegen. . . . . . . . . . . . 120

4.48 Georadarmessung an der Kastanie Kreuzkirche Fulda. . . . . . . . . . . . . . . 121

$5.1 \quad$ Standorteinfluss auf GPR-Messungen. . . . . . . . . . . . . . . . . . . 132

$5.2 \quad$ Beispielhafte Visualisierung einer Georadarmessung mit gpr-slice. . . . . . . . . . 136 
A.1 Versuchsbecken im Neuen Botanischen Garten. . . . . . . . . . . . . . . . . . . . xi

A.2 Lage der Messflächen am Standort Schillerwiese. . . . . . . . . . . . . . . . xii

A.3 Untersuchte Bäume auf der Schillerwiese. . . . . . . . . . . . . . . . . . . xiii

A.4 Untersuchte Bäume am Berliner Landwehrkanal. . . . . . . . . . . . . . . . . . xiv

A.5 Lage der Messflächen am Standort Landwehrkanal Einsteinufer. . . . . . . . . . . xv

A.6 Lage der Messflächen am Standort Landwehrkanal Corneliusufer. . . . . . . . . . xvi

A.7 Untersuchte Bäume auf den weiteren Messflächen. . . . . . . . . . . . . . . . xvii

A.8 Lage der Messfläche am Standort Kastanie Archipelstraat Nimwegen. . . . . . . xviii

A.9 Lage der Messfläche am Standort Kastanie Kreuzkirche Fulda. . . . . . . . . . . xviii

A.10 Lage der Messfläche am Standort Kastanienreihe Göttingen. . . . . . . . . . . . . xix

A.11 Lage der Messfläche Amberbaum Göttingen. . . . . . . . . . . . . . . . . . . . . . xix

A.12 Reproduzierbarkeitsmessung auf der Messfläche Buchengruppe. . . . . . . . . . . xx

A.13 Ergebnis der Georadaruntersuchung auf der Messfläche Schwarzkiefer. . . . . . . xxi

A.14 Ergebnis der Georadaruntersuchung auf der Messfläche Fichtengruppe. . . . . . . . xxii

A.15 Ergebnis der Georadaruntersuchung auf der Messfläche Fichte. . . . . . . . . . . xxiii

A.16 Ergebnis der Georadaruntersuchung auf der Messfläche Buchengruppe. . . . . . . . xxiv

A.17 Ergebnis der Georadaruntersuchung auf der Messfläche Platane . . . . . . . . . . xxv

A.18 Ergebnis der Georadaruntersuchung auf der Messfläche Silberahorn. . . . . . . . xxvi

A.19 Ergebnis der Georadaruntersuchung auf der Messfläche Hybridpappel. . . . . . . xxvii

A.20 Ergebnis der Georadaruntersuchung auf der Messfläche Große Kastanie. . . . . . xxviii

A.21 Ergebnis der Georadaruntersuchung auf der Messfläche Kleine Kastanie. . . . . . xxix

A.22 Ergebnis der Georadaruntersuchung auf der Messfläche Amberbaum. . . . . . . . xxx

A.23 Ergebnis der Georadaruntersuchung auf der Messfläche Kastanien Goldschmidt-

straße $($ Baum 1$) . \ldots \ldots \ldots \ldots \ldots \ldots \ldots \ldots \ldots$. . . . . . . . . . . . . . . . . . .

A.24 Ergebnis der Georadaruntersuchung auf der Messfläche Kastanien Goldschmidtstraße $($ Baum 2). . . . . . . . . . . . . . . . . . . . . xxxii

A.25 Ergebnis der Georadaruntersuchung auf der Messfläche Kastanie Nimwegen. . . . xxxiii

A.26 Ergebnis der Georadaruntersuchung auf der Messfläche Kastanie Fulda. . . . . . xxxiv

A.27 Für die Tiefenumrechnung erfasste Geschwindigkeiten (Standorte 1 und 2). . . . xxxv

A.28 Für die Tiefenumrechnung erfasste Geschwindigkeiten (Standorte 3 und 4). . . . xxxvi

A.29 Ergebnis der Geoelektrikuntersuchung auf der Messfläche Schwarzkiefer. . . . . . xxxvii

A.30 Ergebnis der Geoelektrikuntersuchung auf der Messfläche Fichtengruppe. . . . . xxxviii

A.31 Ergebnis der Geoelektrikuntersuchung auf der Messfläche Buchengruppe. . . . . . xxxix

A.32 Ergebnis der Geoelektrikuntersuchung auf der Messfläche Silberahorn. . . . . . . . xl

A.33 Ergebnis der Geoelektrikuntersuchung auf der Messfläche Hybridpappel. .... xli

A.34 Ergebnis der Geoelektrikuntersuchung auf der Messfläche Große Kastanie. . . . . . xlii

A.35 Ergebnis der Geoelektrikuntersuchung auf der Messfläche Kleine Kastanie. . . . . xliii 



\section{Tabellenverzeichnis}

$2.1 \quad$ Vorarbeiten anderer Autoren zur Wurzeldetektion. . . . . . . . . . . . . 26

3.1 Übersicht über die Messflächen . . . . . . . . . . . . . . . . . . . . . . . . . . 34

3.2 Maße der Messflächen . . . . . . . . . . . . . . . . . . . . . . . . 36

5.1 Anwendbarkeit geophysikalischer Verfahren zur Wurzeldetektion . . . . . . . . . 131

5.2 Merkmale verschiedener Baumstandorte und ihr Einfluss auf GPR-Messungen. . 132

A.1 Dielektrizitätszahlen verschiedener Materialien. . . . . . . . . . . . . . . . . . . vii

A.2 $\quad$ Spezifische Widerstände verschiedener Materialien. Verändert nach Weidelt (2005)]viii

A.3 Anordnung der Wurzelattrappen und Probekörper in den Versuchsbecken 1 bis 4 ix 



\section{Abkürzungsverzeichnis}

A Halbwertsbreite (Breite bei halber Amplitude; entspricht W)

AK Stadtböden Arbeitskreis Stadtböden

B

Bandbreite

BAW

Bundesanstalt für Wasserbau

C

Lichtgeschwindigkeit

CMP Common-Midpoint

CO

Constant-Offset

d

Tiefe

$\varepsilon_{\mathrm{r}}$

Dielektrische Permittivität/ Dielektrizitätskonstante

ERT

Electrical Resistivity Tomography

f

Frequenz

FGSV

Forschungsgesellschaft für Straßen- und Verkehrswesen e.V.

G

Konfigurationsfaktor

GPR

Ground Penetrating Radar

GPS

Global Positioning System

GSSI

Geophysical Survey Systems, Inc.

$\mathrm{Hz}$

Hertz

I

elektrischer Strom

$\lambda$

Wellenlänge

Ld3

mittlere Lagerungsdichte

Lu

schluffiger Lehm

R

elektrischer Widerstand

RAS-LP 4

Richtlinien für die Anlage von Straßen, Teil Landschaftspflege Abschnitt 4

$\mathbf{R}_{G P R}$

Reflexionskoeffizient

$\Delta R_{\text {hor }}$

horizontale Auflösung GPR

$\Delta R_{\text {vert }}$

vertikale Auflösung GPR

$\rho$

spezifischer elektrischer Widerstand

$\rho_{a}$

scheinbarer spezifischer elektrischer Widerstand

$\mathbf{S}$

Weg/ Distanz

elektrische Leitfähigkeit

SI3 lehmiger Sand

Slu lehmig-schluffiger Sand

t Zeit

$\tau \quad$ Pulsbreite

T Periodendauer

$\Theta_{v} \quad$ volumetrischer Wassergehalt 
Abkürzungsverzeichnis

TU Berlin Technische Universität Berlin

U elektrische Spannung

Ut3 toniger Schluff

Ut4 schluffiger Lehm

v Geschwindigkeit

W Pulsbreite bei halber Amplitude; entspricht A

ZTV-Baumpflege Zusätzliche technische Vertragsbedingungen und Richtlinien für Baumpflege 


\section{Einleitung}

Baumwurzeln sind ein wichtiger ökosystemarer Faktor, dessen Bedeutung sich beispielsweise in dem in Wurzelsystemen gespeicherten Kohlenstoff und der Einbindung in weitere ökologische Kreisläufe über die Wasser- und Nährstoffaufnahme zeigt.

Informationen über die Verteilung von Baumwurzeln im Boden sind aber nicht nur aus wissenschaftlichen, sondern auch aus praktischen Gründen beim Management von städtischen Baumbeständen von Interesse.

Auf der praktischen Seite steht dabei, besonders im urbanen Raum, die Wechselwirkung zwischen Mensch und Baum im Vordergrund und kann damit auch als ökosystemarer Zusammenhang aufgefasst werden. Im besiedelten Raum können Informationen über die Verankerung eines Baumes im Boden durch seine Wurzeln helfen, die Bäume zu identifizieren, die eine potentielle Gefahr für die urbane Bevölkerung darstellen.

Außerdem können Kenntnisse über die Lage von Wurzeln auch dazu beitragen, mögliche Gefahren für urbane Bäume zu minimieren, wenn bauliche Eingriffe im städtischen Untergrund geplant sind. Beispielsweise führen Aufgrabungen, bei denen Wurzelsysteme aufgrund von Unkenntnis beschädigt werden, zu verminderter Wasser- und Nährstoffaufnahme und damit zu Vitalitätsdefiziten von geschädigten Gehölzen. Gleichzeitig entstehen an den gekappten Wurzeln Eintrittsstellen für Pathogene, die die Vitalität von Bäumen zusätzlich herabsetzen. Im Fall von Baumpilzen wie dem Riesenporling können solche Pathogene auch wiederum die Verkehrssicherheit eines befallenen Baumes in Frage stellen. Durch den Schutz von Wurzelsystemen städtischer Gehölze werden also gleichzeitig auch keine neuen Gefahrenbäume geschaffen. Weiterhin wird die Vitalität urbaner Gehölze erhalten.

Beispiele für klassische wissenschaftliche Arbeiten über Baumwurzeln sind die Monographie über die Wurzeln der Waldbäume von Köstler et al. (1968) und der Wurzelatlas mitteleuropäischer Waldbäume und Sträucher von Kutschera und Lichtenegger (2002). In diesen Arbeiten wird deutlich, dass die Verteilung von Baumwurzeln inhomogen ist und damit nicht ohne weitergehende Untersuchungen beurteilt werden kann.

In der Wissenschaft werden Wurzelsysteme aber nicht nur als eigenständige Untersuchungsobjekte aufgefasst. Stattdessen wird auch die Rolle von Wurzeln in anderen Disziplinen, z.B. der Bodenkunde, untersucht. Schließlich sind Wurzelsysteme, wie einleitend geschildert, wichtige Speicher von Kohlenstoff im Boden, die bei der Untersuchung von $\mathrm{CO}_{2}$-Flüssen berücksichtigt werden müssen. Auch die angewandte Forschung beschäftigt sich mit Wurzeln. Beispielsweise wird untersucht, wie sich die Durchwurzelung des Bodens bei der Stabilisierung von Hängen und Deichen auswirkt.

Die Durchwurzelung des Bodens wird bislang in der Regel destruktiv durch Beprobungen mit Stechzylindern oder Wurzelbohrern untersucht. Diese Untersuchungsformen haben den Nachteil, dass sie gehölzschädigend sind, in der Regel nur stichprobenartig erfolgen können und durch die 
Materialentnahme die Messbedingungen verändern. Dadurch sind Wiederholungsmessungen und Messreihen unter gleichen Bedingungen unmöglich. Bei praktischen Fragestellungen werden in der Regel aufwendige Aufgrabungen durchgeführt, die kostspielig sind und ebenfalls mit dem Risiko von Baumschädigungen einhergehen.

Der Einsatz zerstörungsfreier geophysikalischer Messverfahren, der in Studien verschiedener Autoren erfolgversprechende Ergebnisse geliefert hat, scheint hier Abhilfe schaffen zu können. Teilweise gehen diese Untersuchungen soweit, mittels bildgebender Verfahren den Kohlenstoffspeicher im Boden zu quantifizieren.

Bisherige Arbeiten haben sich aber vornehmlich auf die Untersuchung einzelner Transekte (2D-Messungen) konzentriert. In der vorliegenden Arbeit soll untersucht werden, ob durch die flächenhafte Aufnahme von Baumstandorten (3D-Messungen) eine bessere Identifikation von Baumwurzeln möglich ist, da gerade im städtischen Raum Schwierigkeiten bei der Unterscheidung von Wurzeln und anderen Objekten bestehen. Dabei liegt der Schwerpunkt der Untersuchungen auf Messungen an realen Baumstandorten (in situ-Messungen). Zur weiteren Etablierung der Verfahren wird insbesondere untersucht, wie sich verschiedene Messkonfigurationen auf die Wurzeldetektion auswirken. Die dadurch geschaffenen Messgrundlagen werden dann auf verschiedenen städtischen Standorten mit zunehmend komplexeren Standortbedingungen angewendet. Eine weitere Arbeitshypothese bei diesen Untersuchungen ist, dass sich die Wurzelsysteme mit zunehmender Komplexität nicht mehr rekonstruieren lassen. 


\section{Stand des Wissens}

\subsection{Baumwurzelsysteme}

\subsubsection{Einteilung und Aufbau}

Funktion Baumwurzeln lassen sich anhand ihrer Funktion in die zwei Klassen: Skelett- und Feinwurzeln einteilen. Feinwurzeln sind dabei der Teil der Wurzeln, dessen Aufgabe in der Wasserund Nährstoffaufnahme besteht. Die Skelettwurzeln fungieren neben dem Weitertransport des aufgenommenen Wassers mit den darin gelösten Nährstoffen, dem Assimilatstrom, auch als Verankerung des Baumes im Boden (Polomski und Kuhn 2001).

Wuchsrichtung Neben dieser funktionellen Einteilung ist im Zusammenhang mit der vorliegenden Arbeit besonders die Einteilung von Wurzelsystemen anhand von dominanten Wuchsrichtungen von Bedeutung. Nach Köstler et al. (1968), die entsprechende Untersuchungen an Waldbäumen durchgeführt haben, unterscheidet man Wurzeln vertikaler und horizontaler Wuchsrichtung, wobei diagonal verlaufende Wurzeln fallspezifisch in eine der beiden genannten Gruppen eingeordnet werden. Die Gesamtheit der Wurzeln wird dabei als Wurzelwerk bezeichnet, wobei synonym auch die Begriffe Wurzelsystem und Wurzeltracht sowie Wurzelhabitus (Polomski und Kuhn 2001, S. 306) verwendet werden können. Ausgangspunkt des Wurzelwerks ist der Wurzelstock, der die unterirdische Verlängerung des Stammes bezeichnet. Nach Köstler et al. (1968) werden die Vertikalwurzeln weitergehend in Herz-, Senker- und Pfahlwurzeln gegliedert. Polomski und Kuhn (2001) verweisen darauf, dass Kutschera und Lichtenegger (2002) für die Pfahlwurzel den Begriff Polwurzel verwenden, da diese direkt aus der Keimwurzel hervorgeht. Pfahlwurzeln bezeichnen die in direkter Verlängerung des Wurzelstocks senkrecht nach unten verlaufenden Wurzeln. Dagegen werden parallel dazu laufende, am Stock ansetzende Wurzeln als Nebenpfahlwurzeln bezeichnet. Schräg verlaufende, am Stock ansetzende Wurzeln fallen unter den Begriff Herzwurzel und die feineren Wurzeln, die von den Horizontalwurzeln senkrecht oder schräg nach unten verlaufen, bilden die Klasse der Senkerwurzeln (Köstler et al. 1968).

Wurzelsystem Abhängig von der vorherrschenden Wuchsrichtung werden auch die gesamten Wurzelsysteme einheimischer Baumarten stark vereinfachend in die drei Haupttypen Herzwurzel-, Senkerwurzel- und Pfahlwurzelsystem (Abbildung 2.1) eingeteilt (Köstler et al. 1968, Kutschera und Lichtenegger 2002, Polomski und Kuhn 1998; Polomski und Kuhn 2001). Aus Kutschera und Lichtenegger (2002) geht allerdings auch hervor, dass diese idealtypisch ausgebildeten Wurzelsysteme unter natürlichen Bedingungen in der Regel nicht vorgefunden werden.

Altersphasen von Wurzelsystemen Das Wurzelsystem eines Baumes ist das Resultat eines langen Wachstumsprozesses, demzufolge seine Form Änderungen unterliegt . Polomski und Kuhn 


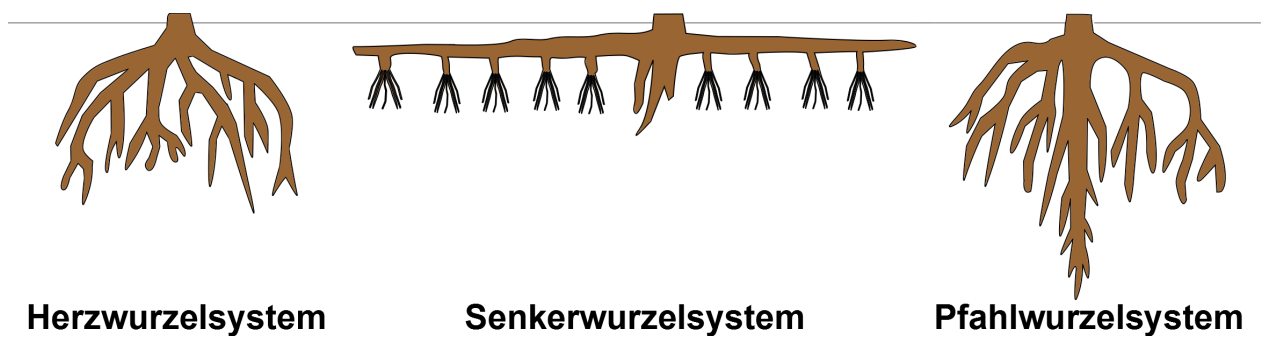

Abbildung 2.1: Idealisierte, stark vereinfachte Einteilung der Wurzelsysteme einheimischer Baumarten. Verändert nach Köstler et al. (1968), Kutschera und Lichtenegger (2002), Polomski und Kuhn (1998) sowie Polomski und Kuhn (2001). Aus den Ausführungen von Kutschera und Lichtenegger (2002) geht allerdings hervor, dass diese idealen Wurzeltypen unter natürlichen Bedingungen in der Regel nicht vorgefunden werden.

(2001, S. 304 f.) fassen diesbezüglich zusammen, dass sich in der Entwicklung eines Wurzelsystems vier Phasen unterscheiden lassen. Die erste Phase wird als zwei- bis dreijährige „PfahlwurzelPhase" (Polomski und Kuhn 2001, S. 304) bezeichnet, an die sich eine etwa zehnjährige Phase anschließt, in der sich vertikale, horizontale und schrägwachsende Wurzeln zunehmend herausbilden und insbesondere die Horizontalwurzeln ein Längen- und Dickenwachstum aufweisen. In der dritten Phase werden entsprechend der genetischen Veranlagung Senkerwurzeln ausgebildet. Während dieser Phase verlangsamt sich das Tiefenwachstum und die Vertikalerschließung ist bei einem Baumalter von etwa 40 Jahren abgeschlossen. In der sich daran anschließenden vierten Phase werden „neue Senker, Wurzelanläufe oder Brettholz [...] nach individuellem Bedarf und Standortsangebot gebildet" (Polomski und Kuhn 2001, S. 305). Des Weiteren wird von Polomski und Kuhn (2001) insbesondere auf die mögliche Adventivwurzelbildung am Stamm in dieser Entwicklungsphase hingewiesen. Ein weiteres Kennzeichen älterer Wurzelsysteme ist die „herabgesetzte Resistenz gegenüber Krankheitserregern“ (Polomski und Kuhn 2001, S. 305). Diese kann sich aus der langjährigen Aufnahme von Schadstoffen und Pathogenen ergeben.

Größeneinteilung von Wurzeln Neben der Einteilung nach der Wuchsrichtung einzelner Wurzeln, des gesamten Wurzelsystems und ihrer Funktion werden die einzelnen Wurzeln eines Baumes verschiedenen Größenklassen zugerechnet. Entsprechende Einteilungen verschiedener Autoren werden beispielsweise bei Kutschera und Lichtenegger (2002) zusammengefasst. Abbildung 2.2 zeigt die Einteilungen nach Köstler et al. (1968) und der ZTV-Baumpflege (FLL 2006). Köstler et al. (1968) ziehen die Grenze zwischen Fein- und Grobwurzeln bei einem Durchmesser von $5 \mathrm{~mm}$, wobei diese beiden Klassen jeweils noch genauer differenziert werden. Dabei wird jeweils auch eine Größenklasse als „Fein- und Grobwurzeln im engeren Sinne“ (Köstler et al. 1968, S. 12) ausgewiesen. In der baumpflegerischen Praxis wird bei Eingriffen im Wurzelraum in der Regel die Einteilung nach der ZTV-Baumpflege (FLL 2006) herangezogen. Darin sind auch entsprechende Vorgaben für die Durchführung von Arbeiten im Wurzelbereich formuliert. Weitere Richtlinien zum Wurzelschutz bei derartigen Baumaßnahmen finden sich in der RAS-LP 4 (FGSV 1999). 

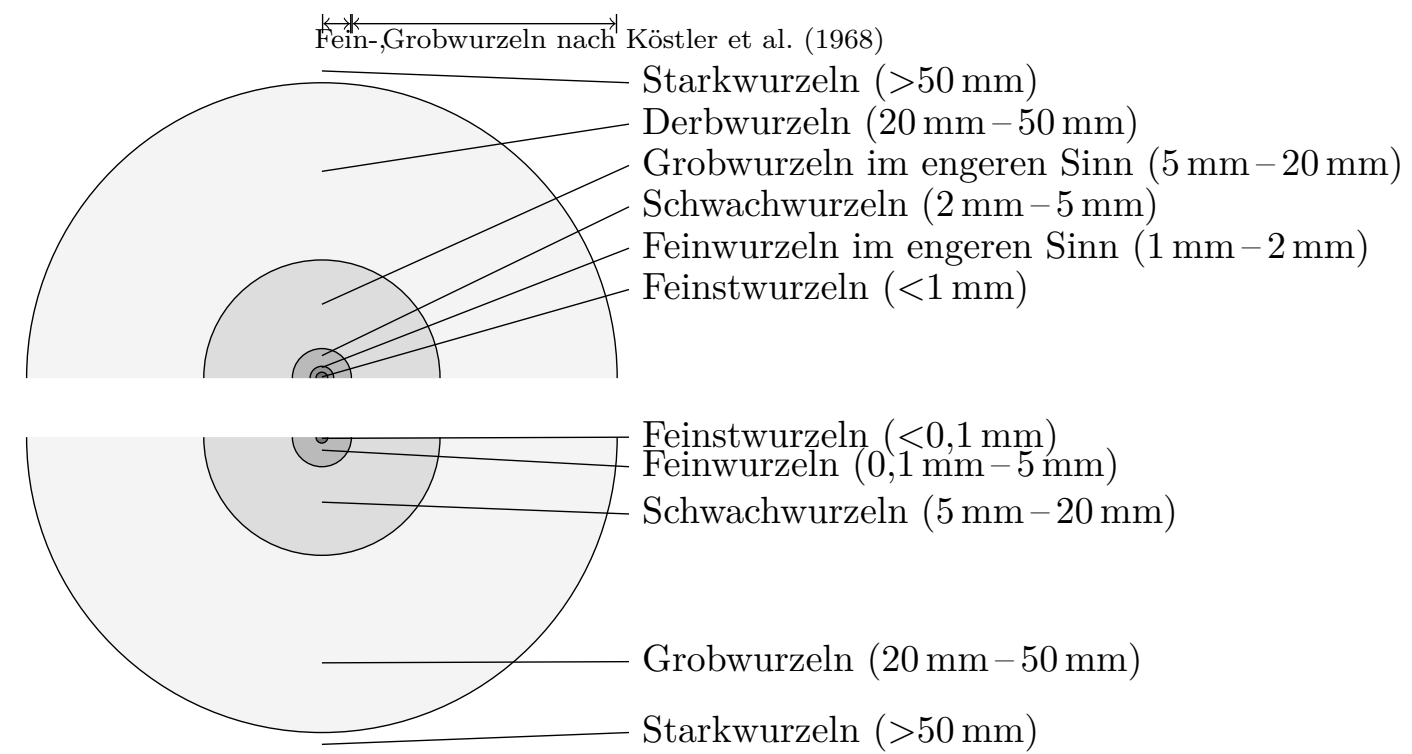

Abbildung 2.2: Einteilung von Wurzeln in unterschiedliche Größenklassen nach Köstler et al. (1968) (oben) und der ZTV-Baumpflege (FLL 2006) (unten).

\subsubsection{Wurzelsysteme in Abhängigkeit von Baumart und Standort}

Bei der Ausbildung eines Wurzelsystems wirken Kutschera und Lichtenegger (2002) zufolge zeitgleich verschiedene Faktoren. Dabei handelt es sich um „genetische, klimatische oder edaphische Einflüsse" (Kutschera und Lichtenegger 2002, S. 48). Dadurch, dass all diese Faktoren stets gleichzeitig auftreten, ist es nicht möglich, die Ausbildung eines Wurzelsystems einer einzelnen dieser Größen zuzuordnen. Es kann lediglich festgestellt werden, welcher Einfluss überwiegt. Dementsprechend werden bei Kutschera und Lichtenegger (2002, S. 49) „vorwiegend durch Erbanlagen bedingte Wurzelformen" und „vorwiegend durch die Umwelt bedingte Wurzelformen“ unterschieden. Bezüglich Letzterer heißt es bei Kutschera und Lichtenegger (2002, S. 49) weiter: „Innerhalb der artspezifischen Wandelbarkeit der Bewurzelung wirken sich die Umwelteinflüsse entscheidend auf die Bildung der Wurzelformen aus. Zum Ausdruck kommt dies besonders in der standortbedingten Verteilung der Wurzelmasse im Boden [...]. In welcher Weise diese erfolgt, hängt in erster Linie vom Wärme- und Wasserhaushalt und schließlich auch von den Bodeneigenschaften ab.“

Im urbanen Raum treten zu den natürlichen Einflussfaktoren auf die Wurzelentwicklung weitere hinzu. Balder (1998, S. 25) merkt diesbezüglich an, dass „die Wurzeln urbaner Gehölze [...] in Stadtböden zahlreichen Beeinträchtigungen ausgesetzt [sind]“. Dazu gehören Schadstoffeintrag, eine veränderte Nährstoffversorgung, Bodenversiegelungen und -verdichtungen, eine eingeengte Baumgrube sowie Eingriffe in den baumnahen Untergrund. Diese führen dazu, dass in städtischen Böden „häufig [...] keine gleichmäßige und artgerechte Wurzelentwicklung mehr [erfolgt]“ (Balder 1998, S. 25). Weiterhin stelle „die Ausbildung [...] des Wurzelwerks jeweils die Summenreaktion aller beeinflussender Faktoren dar" (Balder 1998, S. 25).

Dazu merken Polomski und Kuhn (2001, S. 307) an, dass der ,ideale Wurzelphaenotyp [...] als Bezugsgröße für vergleichende wissenschaftliche Untersuchungen von Bedeutung [wäre]. Unter 
den Bedingungen der Praxis kann jedoch das Wurzelsystem nur bei vorherrschenden Bäumen in sandigen oder sonst lockeren Böden [...] eindeutig den idealen Typen zugeordnet werden.“ (Polomski und Kuhn 2001, S. 307).

Daher kann in der Praxis ohne weitergehende Untersuchungen weder aufgrund der Baumart noch aufgrund des Standorts eine gesicherte Aussage zur Morphologie des Wurzelsystems getätigt werden. Bei Gehölzen, deren Wurzelsystem beispielsweise in Baumschulen für eine spätere Pflanzung gezielt präpariert worden ist, ist der Wurzelballen durch diese Verschulung zusätzlich verändert. Dies führt dazu, dass mit einer gewissen Sicherheit eine hohe Dichte von Wurzeln in Baumnähe erwartet werden kann. Wie sich der Baum mit seinen Wurzeln an die Bedingungen am neuen Standort anpasst, bleibt allerdings weitgehend unvorhersehbar.

Bezüglich der vorliegenden Arbeit ist einerseits die genannte Einteilung in verschiedene Größenklassen von besonderer Bedeutung (beispielsweise bei der Ermittlung der Auflösungsmöglichkeiten der eingesetzten Verfahren - vgl. Kapitel 2.3.2.2). Andererseits müssen morphologische Kriterien herangezogen werden, um die untersuchten Wurzelsysteme zu beschreiben und zu beurteilen (vgl. Kapitel 4.2 und 4.3. Außerdem ergibt sich aus der unvorhersehbaren Wurzelverteilung im Boden ein Grund für den Einsatz geophysikalischer Methoden zur Wurzeldetektion.

\subsection{Stadtböden}

Der einfachsten Definition folgend sind städtische Böden die Böden, die sich innerhalb städtischer Grenzen befinden. Meuser und Blume (2011) unterscheiden dabei aus anthropogen abgelagerten Ausgangsmaterialien und aus natürlichen Substraten hervorgegangene Böden. Bei den natürlichen Substraten sind dabei wesentliche Eigenschaften durch die anthropogene Nutzung verändert worden. Nach den Empfehlungen des AK Stadtböden (1997) werden „urban, gewerblich, industriell oder montan überformte Böden [...] auch als Stadtböden bezeichnet" (Meuser und Blume 2011, S. 465). Böden, deren Morphe über $40 \mathrm{~cm}$ hinaus durch anthropogene Eingriffe verändert worden ist, werden klassifikatorisch als Anthropogene Böden bezeichnet (Meuser und Blume 2011).

Beim Ausgangsmaterial von Böden in Ballungsräumen lassen sich natürliche mineralische und organische Substrate, die umgelagert worden sind und technogene Substrate unterscheiden. Technogene Substrate sind dabei künstlich hergestellte Materialien oder Stoffe, deren Urzustand entscheidend modifiziert wurde (Meuser und Blume 2011). Beispiele sind Ziegel, Beton, Mörtel, Schlacke, Müll, Klärschlamm und Asche. Anhand der Städte Stuttgart (Holland 1996) und Essen (Meuser 1996) lässt sich exemplarisch der hohe Anteil technogener Substrate in Stadtgebieten erkennen. In beiden Fällen weist über die Hälfte der untersuchten Böden rein technogene Substrate oder Mischformen aus natürlichen und technogenen Substraten auf. Da anthropogene Böden durch häufige Eingriffe und Umlagerungen in der Regel Böden in einem nicht sehr weit fortgeschrittenen Entwicklungsstadium sind, dominieren die technogenen Ausgangssubstrate in der Regel die Eigenschaften des gesamten Bodens. Ein häufig für Aufschüttungen verwendetes Substrat ist beispielsweise Bauschutt, der in der Regel einen erhöhten Schwermetallgehalt aufweist (Hiller und Meuser 1998).

Dies ist im Rahmen der vorliegenden Arbeit von Interesse, da solche Substrate oft höhere 
elektrische Leitfähigkeiten aufweisen, welche die durchgeführten Messungen beeinflussen können. Ähnliche Beeinflussungen können auch durch Salzeinträge im Rahmen von Streumaßnahmen (Winterdienst) hervorgerufen werden (Hiller und Meuser 1998). Entsprechend erhöhte Leitfähigkeiten im Oberboden können beispielsweise die Sondierungstiefen der eingesetzten Messverfahren herabsetzen.

Weiterhin kann die Grobkörnigkeit des Substrats bei solchen künstlich aufgeschütteten Bauschuttgemengen einen wesentlichen Einfluss auf die Messungen haben. Das ist der Fall, wenn einzelne Komponenten eine Größe im Bereich der eigentlichen Zielobjekte der Messungen (den Baumwurzeln) aufweisen. Dies kann sich in der Weise auswirken, dass anstelle der zu ortenden Wurzeln andere Strukturen die Visualisierungsergebnisse dominieren.

Neben den speziellen Eigenschaften von Stadtböden ist die kleinräumige Heterogenität ein Einflussfaktor auf die durchgeführten Untersuchungen. So ist es in der Stadt schwer möglich, durch die Ermittlung von Bodeneigenschaften an einzelnen Probepunkten Aussagen über den Untergrundaufbau einer größeren Fläche zu treffen. Veränderungen durch kleinräumige anthropogene Eingriffe (z.B. im Rahmen von Leitungs- und anderen Infrastrukturarbeiten) können bei stichprobenartigen Untersuchungen nicht unbedingt festgestellt werden.

\subsection{Geophysikalische Untersuchungsverfahren}

\subsubsection{Grundlagen elektromagnetischer Methoden}

Weidelt (2005) fasst unter dem Begriff Geoelektrik verschiedene elektrische und elektromagnetische Verfahren zusammen, die eingesetzt werden, um Änderungen folgender Untergrundeigenschaften zu bestimmen:

- der elektrischen Leitfähigkeit (Kehrwert des elektrischen Widerstands)

- der Polarisationseigenschaften und des Aufladevermögens

- der dielektrischen Eigenschaften

- der natürlichen elektrischen Felder

Nach dieser Definition wird auch das Georadar, das Änderungen der dielektrischen Eigenschaften untersucht, als eines der elektromagnetischen Verfahren zu den geoelektrischen Untersuchungsverfahren gezählt. Dagegen wird in der vorliegenden Arbeit eine vereinfachende Begriffstrennung genutzt und nur die Gleichstromgeoelektrik als Geoelektrik bezeichnet.

Die Abgrenzung zwischen elektrischen und elektromagnetischen Verfahren kann Weidelt (2005) zufolge anhand des eingesetzten Signals und dessen Erzeugung vorgenommen werden (elektrische Verfahren nutzen Gleich- und Wechselströme mit Frequenzen bis $50 \mathrm{~Hz}$, während elektromagnetische Verfahren mit zeitlich variierenden, auch impulsartigen Anregungen arbeiten und je nach Methode elektrische oder auch magnetische Felder bestimmen). Ein anderes Unterscheidungskriterium ist die Art der Energiezufuhr in Form von galvanischen (Erdung über Elektroden bei 
elektrischen Verfahren) und induktiven Ankopplungen (Antennen und Spulen bei elektromagnetischen Verfahren). Weidelt (2005) merkt hierzu an, dass aber auch bei den elektrischen Methoden induktive Komponenten über die Magnetfelder der Stromkabel vorhanden sind.

Die genaue Entstehung und Ausbreitung von elektromagnetischen Wellen wird durch die Maxwell'schen Gleichungen beschrieben (siehe dazu z. B. Weidelt (2005, S. 74 ff.). Auf eine umfassende theoretische Darstellung der entsprechenden physikalischen Grundlagen wird an dieser Stelle verzichtet, da die vorliegende Arbeit als anwenderorientierte Nutzung von Verfahren zu verstehen ist, die für andere Fragestellungen bereits etabliert sind. Dementsprechend bleibt dieses Kapitel auf die anschauliche Erläuterung der für das weitere Verständnis der Arbeit notwendigen Grundlagen beschränkt. Wichtig ist dabei ein grundlegendes Verständnis dafür, dass mit den beiden eingesetzten Verfahren bestimmte Möglichkeiten bestehen, die gewünschten Informationen zu erlangen, dies aber nur innerhalb der bestehenden physikalischen Grenzen der Methoden möglich ist.

Cassidy (2009, S. 41) betont für den Fall des Bodenradars „dass ein GPR-Schnitt nicht ein Abbild oder Bild des Untergrunds per se ist, sondern stattdessen die zeitabhängige, aufgezeichnete Antwort der Untergrundmaterialien auf die Ausbreitung elektromagnetischer Energie [...]“ (Cassidy 2009, S. 41)(Übs. M.V.).1

Ähnliches gilt entsprechend angepasst auch für das Verfahren der elektrischen Widerstandstomographie. Weidelt (2005, S. 71) schreibt hierzu:,ZZiel der Messungen ist es, aus den ermittelten Parameterverteilungen die Strukturen des Untergrunds und ihre stofflichen Ursachen zu rekonstruieren. Die Aussagesicherheit wird erhöht durch die kombinierte Messung mehrerer geoelektrischer Parameter und durch die Einbeziehung von Randbedingungen aus der Geologie, aus Bohrungen und anderen geophysikalischen Verfahren."

\subsubsection{Georadar}

\subsubsection{Messmethodik}

Das Geo- oder auch Bodenradar (engl. Ground Penetrating Radar; kurz GPR) ist ein geophysikalisches Prospektionsverfahren, das auf dem Einstrahlen aktiv erzeugter, hochfrequenter elektromagnetischer Wellen in den Untergrund basiert (Frequenzbereich von $10 \mathrm{MHz}-1000 \mathrm{MHz}$ nach Annan (2009); Goodman et al. (2009) nennen aber beispielsweise auch 4 GHz-Antennen). Nach der einleitend angeführten Einteilung von Weidelt (2005) gehört das Georadar zu den elektromagnetischen Sondierungsverfahren.

Alteköster (2004) zufolge handelt es sich bei dem Georadar um die geophysikalische Untersuchungsmethode mit der höchsten räumlichen Aufösung. In der Praxis wird es für verschiedene Anwendungen eingesetzt. Neben der wissenschaftlichen Nutzung im Bereich von Bodenkunde, Hydrologie, Glaziologie, Geologie, etc. findet es im kommerziellen Einsatz bei der Ortung von Objekten (Leitungen, Hohlräumen, etc.) Verwendung.

\footnotetext{
${ }^{1}$ Im Original:,,[...] that a GPR-section is not a picture or an image of the subsurface per se but is, instead, the time-dependent, recorded response of the subsurface materials to the propagation of electromagnetic (EM) energy [...]"(Cassidy 2009, S. 41)
} 


\section{Oberflächenreflexion}

Nachschwinger

Reflexion an 1. Grenzschicht (z.B. Wurzel)

Reflexion an 2. Grenzschicht (z.B. Bodenhorizont)

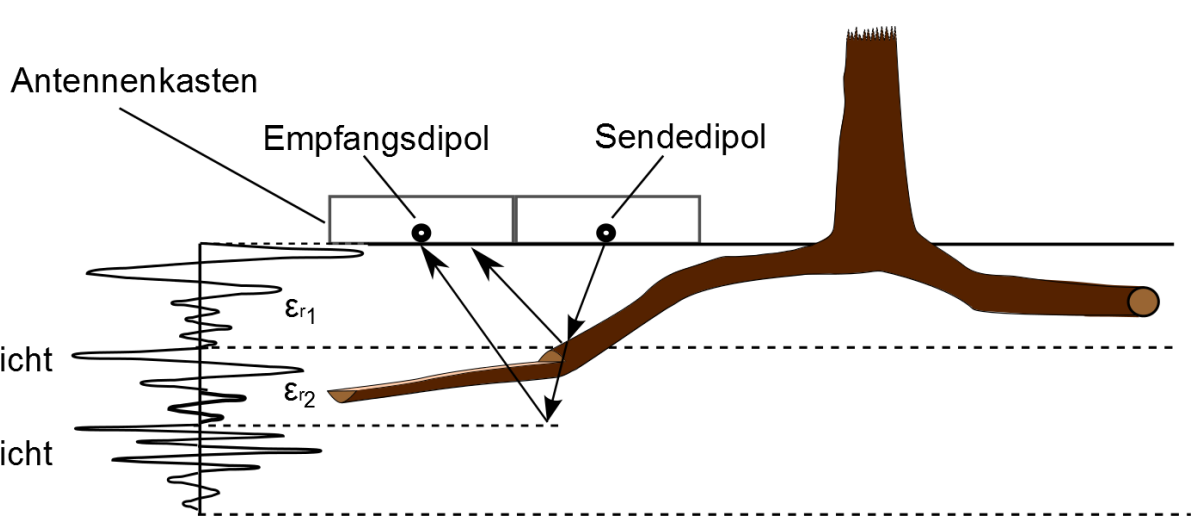

Abbildung 2.3: Messprinzip des Bodenradars bei der Wurzelortung- verändert nach Alteköster (2004).

Beim Georadar werden in einer Steuereinheit hochfrequente elektromagnetische Impulse erzeugt. Diese werden an eine Sendeantenne geleitet und von dieser als gerichtete Impulse in den Untergrund abgestrahlt, wo sie sich als freie Wellen fortsetzen. Je nach Bodenbeschaffenheit kommt es zur Absorption, Transmission, Refraktion und Reflexion der Wellen. Der dabei reflektierte Anteil der Radarwellen wird mit einer Empfangsantenne wieder aufgezeichnet (Daniels 2000: Daniels 2004 Annan 2009).

In Abbildung 2.3 ist diese Funktionsweise exemplarisch für die Anwendung bei der Ortung von Baumwurzeln skizziert.

Generell ist das Auftreten einer Reflexion an die Änderung der dielektrischen Permittivität (auch Dielektrizitätskonstante) $\varepsilon_{\mathrm{r}}$ im Untergrund gebunden. Solche Bereiche werden als dielektrische Grenzschichten bezeichnet. Die Intensität einer Bodenradarreflexion beschreibt Gleichung 2.1.

$$
R_{G P R}=\frac{\sqrt{\varepsilon_{r 1}}-\sqrt{\varepsilon_{r 2}}}{\sqrt{\varepsilon_{r 1}}+\sqrt{\varepsilon_{r 2}}}
$$

$\mathrm{R}_{G P R}$ bezeichnet dabei den Reflexionskoeffizienten; $\varepsilon_{r 1}$ und $\varepsilon_{r 2}$ geben die Dielektrizitätskonstanten der beiden Materialien an der dielektrischen Grenzschicht an (vgl. Abbildung 2.3). Dabei ist das Vorzeichen des Reflexionskoeffizienten davon abhängig, ob ein Materialwechsel zu einer größeren oder zu einer kleineren Dielektrizitätskonstante hin stattfindet. Am Betrag des Reflexionskoeffizienten wird deutlich, wie stark die Reflexion im Verhältnis zum Ausgangssignal ist.

Abhängig von der Messkonfiguration werden beim Georadar entweder eine (monostatische Messungen) oder zwei getrennte Antennen (bistatische Messungen) genutzt. Beim Einsatz von zwei getrennten Antennen lassen sich wiederum verschiedenen Messverfahren realisieren. Typisch sind dabei Messungen mit einem festen (Constant Offset Messungen; CO) oder zunehmenden (Common Midpoint Messungen; CMP) Antennenabstand.

Je nach Dimension der Radaruntersuchung werden A-, B- und C-Scans unterschieden. Bei einem A-Scan handelt es sich um einen einzelnen Tiefenscan, der an einem Punkt aufgenommen wird. Mit dem Begriff B-Scan (auch Transekt, Linescan oder 2D-Radargramm) wird die Aufnahme eines einzelnen Transekts bezeichnet, bei der entlang einer bestimmten Strecke eine vorgegebene Anzahl von A-Scans aufgezeichnet wird. Ein C-Scan beinhaltet die Erfassung einer Fläche und setzt sich 
dementsprechend wiederum aus mehreren B-Scans zusammen. Ein C-Scan kann theoretisch aber auch aus mehreren A-Scans erstellt werden ((vgl. Daniels 2004, S. 249) und Abbildung 2.4).

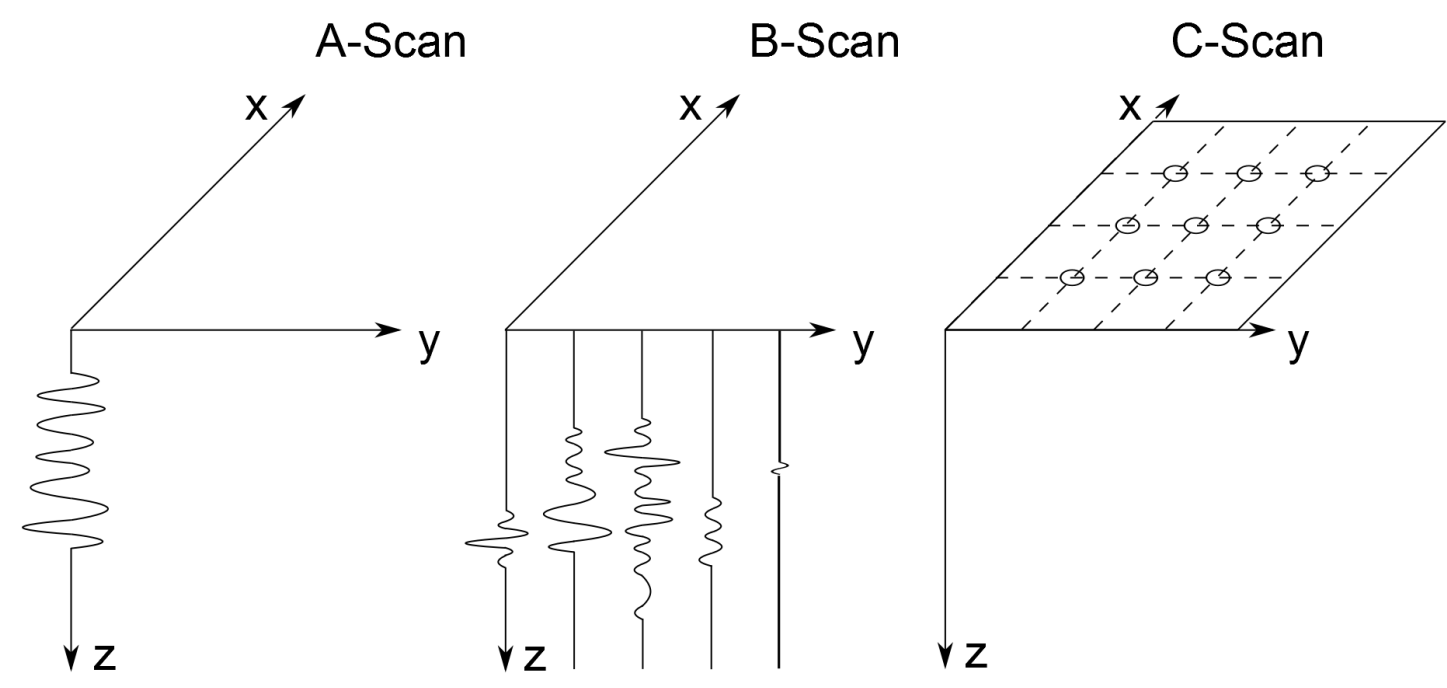

Abbildung 2.4: A-, B- und C- Scans bei Bodenradarmessungen. Verändert nach Daniels (2004, S. 249) Abbildung 7.1.

Wie eingangs erläutert, werden bei einer Georadarmessung im Untergrund reflektierte elektromagnetische Wellen mit einer Empfangsantenne aufgezeichnet (vgl. Abbildung 2.3). Gemessen wird dabei, die Zwei-Wege-Laufzeit von der Abstrahlung an der Sendeantenne bis zur Aufzeichnung an der Empfangsantenne sowie die Amplitude (Stärke) des empfangenen Signals zur jeweiligen Zwei-Wege-Laufzeit. Von Interesse ist zumeist aber nicht die Laufzeit $t$ sondern die Tiefe $s$, in der sich ein bestimmter Reflektor befindet. Die Zwei-Wege-Laufzeiten bis zu einer bestimmten Bodentiefe hängen von der Geschwindigkeit $v$ der Radarwellen im Untergrund ab. Die Umrechnung der Zwei-Wege-Laufzeiten in eine Tiefenangabe erfolgt entsprechend Gleichung 2.2 .

$$
s=\frac{v t}{2}
$$

Dafür muss folglich die Geschwindigkeit $v$ bekannt sein, mit der sich die elektromagnetischen Wellen im Untergrund fortsetzen. Für die Geschwindigkeit von elektromagnetischen Wellen in einem Medium gilt Formel 2.3 .

$$
v=\frac{c}{\sqrt{\varepsilon_{\mathrm{r}}}}
$$

$\varepsilon_{\mathrm{r}}$ bezeichnet dabei die dimensionslose Dielektrizitätskonstante (auch dielektrische Permittivität); c die Lichtgeschwindigkeit. $\varepsilon_{\mathrm{r}}$ kann als vom Ausbreitungsmedium abhängiger Dämpfungsfaktor für die Geschwindigkeit von elektromagnetischen Wellen, die sich im Vakuum mit Lichtgeschwindigkeit $c$ fortsetzen, angesehen werden. Da die $\varepsilon_{\mathrm{r}}$-Werte aller anderen Materialien größer als die von Luft (bzw. dem Vakuum) sind, ist die Geschwindigkeit in diesen immer kleiner als $c$, bzw. die Laufzeit pro m ist höher (Daniels 2004). Variationen der Dielektrizitätskonstante innerhalb eines während einer einzelnen Messung erfassten Areals führen zu Verzerrungen bei der Transformation der Laufzeiten in die entsprechenden Tiefenstufen, da in der Regel nur ein Durchschnittswert für 
den gesamten Laufzeitbereich angegeben werden kann. Da die Dielektrizitätskonstante meist unbekannt ist, lassen sich solche Verzerrungen aber nicht vermeiden.

\subsubsection{Auflösung}

Vertikale Auflösung Die Kenntnis der Auflösung ist zum einen für die weitere Interpretation von Ergebnissen im Anschluss an eine Messung essentiell. Wichtiger ist sie aber in der Regel für eine Einschätzung der Auflösungsgrenzen im Vorfeld einer Messung, um realistische Zielvorgaben zu erhalten.

Ein Kriterium für die vertikale Auflösung, das auch bei Alteköster (2004) angesprochen wird, ist das aus der Optik entlehnte Rayleigh-Kriterium (siehe hierzu z.B. Hering et al. 2004). Dieses besagt, dass zwei Objekte mit gleicher Amplitude anhand der von ihnen reflektierten Signale noch unterschieden werden können, wenn das Maximum des einen Signals mit dem Minimum des anderen zusammentrifft und somit die Laufzeitdifferenz zwischen beiden Signalen der halben Periodendauer T/2 entspricht. Ein anderes, von Sperner (1999) angesprochenes,

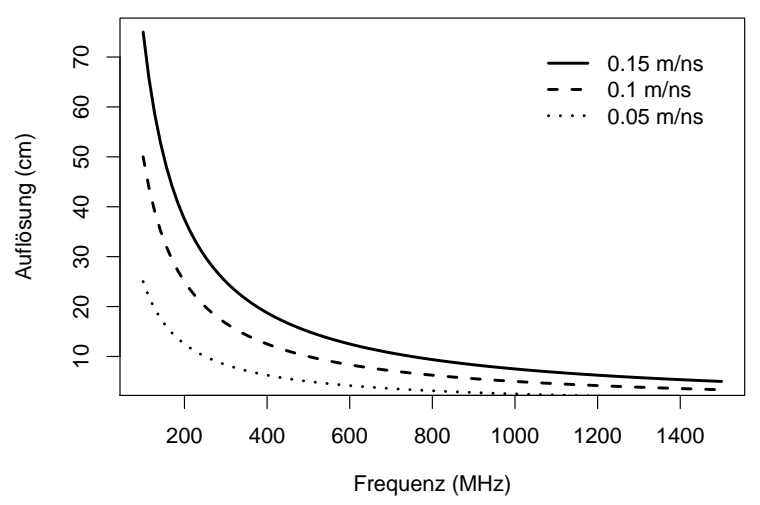

Abbildung 2.5: Vertikale Auflösung bei Bodenradarmessungen; mit Gleichung 2.4 für unterschiedliche Geschwindigkeiten und Antennenfrequenzen berechnet.

Unterscheidungsmerkmal ist das Ricker-Kriterium, demzufolge sich zwei Objekte auch noch differenzieren lassen, wenn sich die von ihnen reflektierten Signale bis zur Halbwertsbreite A angenähert haben. Laut Sperner (1999) lassen sich aber „allgemeingültige Aussagen [...] mit solchen Modellen, die abhängig von der Signalform sind, leider nicht treffen" (Sperner 1999, S. 11). Sperner (1999) zufolge sind sie im Vorfeld von Messungen allerdings sinnvoll, um die Auflösungsgrenzen einzuschätzen. Das Kriterium, mit dem sich die sicherste Unterscheidung treffen lässt, ist die Pulsbreite $\tau$ (Kehrwert der Bandbreite B), des vom Radarsystem abgestrahlten Signals. Da vom Bodenradar Zwei-Wege-Laufzeiten registriert werden, lautet die resultierende Gleichung für die Ermittlung der vertikalen Auflösung $\Delta R_{v e r t}$ nach Alteköster (2004):

$$
\Delta R_{\text {vert }}=\frac{v \tau}{2}=\frac{v}{2 B}
$$


oder nach Annan (2009):

$$
\Delta R_{v e r t} \geq \frac{W v}{4}
$$

Hieraus geht hervor, dass mit steigender Geschwindigkeit $v$ die vertikale Auflösung sinkt, während eine größere Bandbreite $B$ die Auflösung steigert (vgl. Alteköster 2004 und Abbildung 2.5).

Horizontale Auflösung Für die horizontale Auflösung $\Delta R_{\text {hor }}$ (Abbildung 2.6 gilt bei Bodenradarmessungen nach Alteköster (2004):

$$
\Delta R_{h o r}=\sqrt{2 d \lambda+\frac{\lambda^{2}}{4}}
$$

oder nach Annan (2009):

$$
\Delta R_{h o r}=\sqrt{\frac{d \lambda}{2}}
$$

$\Delta R_{\text {hor }}$ entspricht dabei dem Durchmesser der Fresnelschen Zone (vgl. Abbildung 2.6a). Der Mindestabstand, den zwei Objekte zueinander aufweisen müssen, damit sie unterschieden werden können, hängt also von der Wellenlänge und der Tiefe ab, in der sich das zu erfassende Objekt befindet. Die Wellenlänge $\lambda$ ergibt sich aus dem Quotienten von Geschwindigkeit $v$ und Messfrequenz $f$ :

$$
\lambda=\frac{v}{f}
$$

Während die Frequenz im Wesentlichen durch das eingesetzte Messequipment vorgegeben wird, hängt die Geschwindigkeit stark von der dielektrischen Permittivität $\varepsilon_{\mathrm{r}}$ bei den jeweiligen Bodenverhältnissen ab (Gleichung 2.3. .
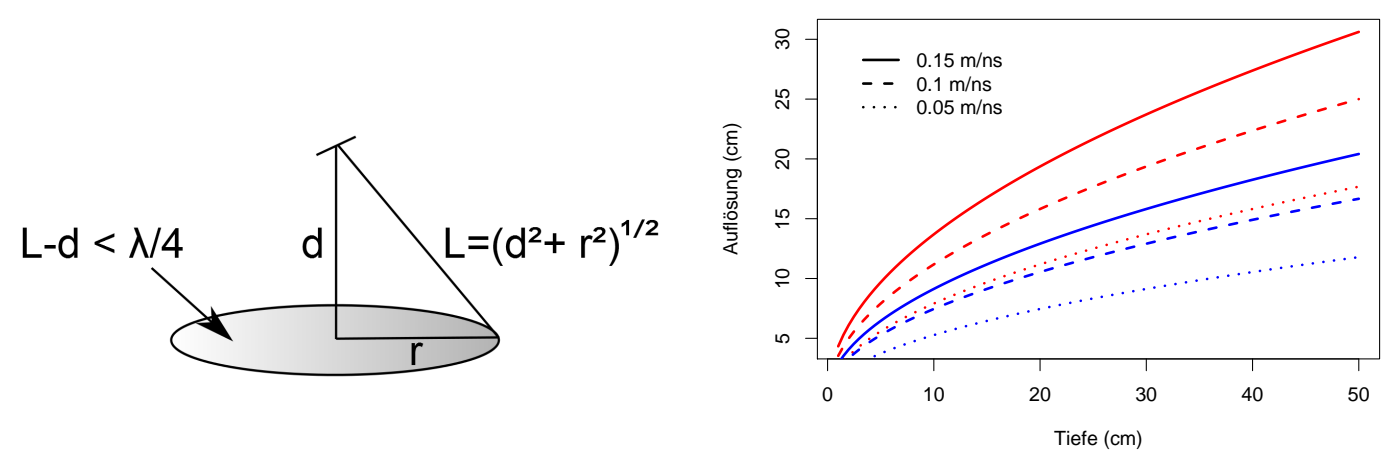

a) Die Fresnelsche Zone bestimmt die horizonta- b) Horizontale Auflösung beim Bodenradar. le Auflösung beim Georadarverfahren. Verändert $400 \mathrm{MHz}$ (rot); $900 \mathrm{MHz}$ (blau) nach Alteköster (2004, S. 40), Abbildung 4.11.

Abbildung 2.6: Horizontale Auflösung bei Bodenradarmessungen; mit Gleichung 2.7 für verschiedene Antennenfrequenzen, Geschwindigkeiten und Bodentiefen berechnet.

Für die jeweilige Untersuchungstiefe $d$ folgt aus Formel 2.7 dass die horizontale Auflösung mit zunehmender Tiefe generell abnimmt (vgl. Abbildung 2.6b). 
Bei konstanter Ausbreitungsgeschwindigkeit $v$, die an einem Messpunkt bei gleicher Bodenfeuchte zu erwarten ist, gilt, dass mit einer höheren Frequenz eine bessere Auflösung erreicht wird. Dies resultiert daraus, dass eine Frequenzerhöhung mit einer Reduzierung der Wellenlänge einhergeht, welche gleichzeitig allerdings auch eine Reduzierung der Eindringtiefe bewirkt. Für die praktische Durchführung von Bodenradarmessungen folgt daraus wiederum, dass je nach Untersuchungsziel unterschiedliche Antennen gewählt werden müssen. Dies liegt daran, dass eine Bodenradarantenne üblicherweise Wellen einer bestimmten (Mitten-)frequenz abstrahlt. Die Wahl der jeweiligen Antenne ermöglicht aber nicht beliebig gute Auflösungen in jeder Untersuchungstiefe. Es können entweder bodennahe hochauflösende oder tiefer gehende Messungen mit geringerer Auflösung durchgeführt werden.

Umlaufende Wellen Ein Spezialfall ist das Auftreten von sogenannten umlaufenden Wellen. Die Entstehung dieser Wellen wird bei Raschik (2005) (dort „Geführte Wellen“ Raschik 2005 , S. 28) erläutert. Demnach laufen diese Wellen nicht von der Sendeantenne zur Objektoberkante und dann weiter zur Empfangsantenne (Abbildung 2.7 direkte Reflexion), sondern umlaufen das Objekt im Boden und treffen erst danach auf die Empfangsantenne (Abbildung 2.7; Umlaufende Welle). Deswegen ist die Laufzeit im Vergleich zu direkt reflektierten Wellen länger.

Das Auftreten von umlaufenden Wellen ist grundsätzlich auch bei Bodenradarmessungen zur Wurzelortung denkbar. Dabei spielt die vertikale Auflösung eine wichtige Rolle, da diese so groß sein muss, dass die umlaufende Welle aufgelöst werden kann und nicht vom Signal der direkten Reflexion überlagert wird.

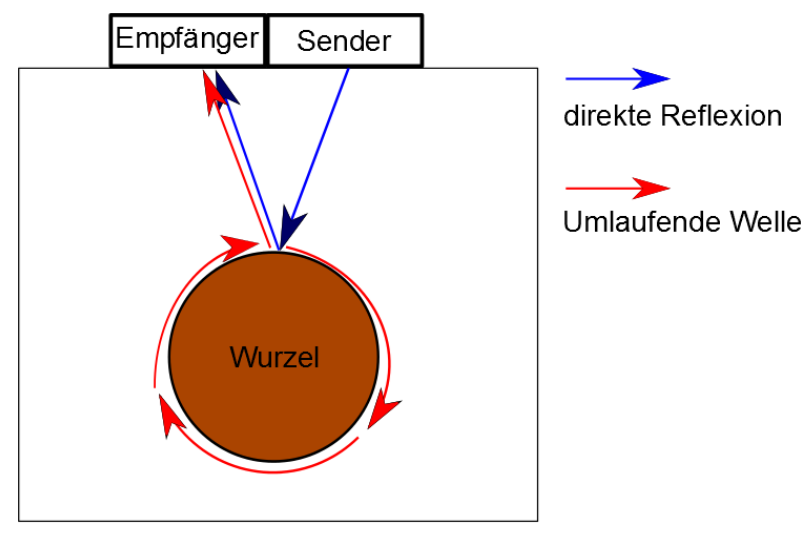

Abbildung 2.7: Umlaufende Welle und Zwei-Wege-Reflexion an Wurzeln. Verändert nach Obermeier und Sodeikat (2012, S. 4), Bild 3.

Beispielsweise nutzen Naser und Junge (2012) umlaufende Wellen, um die Ergebnisse von Bodenradarmessungen bei der Leitungsortung zu erklären. Ebenfalls im Anwendungsfeld von Untersuchungen an Rohrleitungen verwenden Obermeier und Sodeikat (2012) diesen Erklärungsansatz, um Reflexionswege bei Ultraschallmessungen an Rohrleitungen zu rekonstruieren. 


\subsubsection{Bearbeitung von Georadardaten}

Neben den im Idealfall in den Daten enthaltenen Informationen über die Zielobjekte werden auch unerwünschte Signale (sogenannter noise) aufgezeichnet. Dazu zählen beispielsweise elektromagnetische Wellen von Mobiltelefonen, Antennenrauschen oder Störungen im Radargramm, die auf eine schlechte Ankopplung der Antenne an den Untergrund zurückzuführen sind (Olhoeft 2000; Conyers 2004).

Ziel einer Georadaruntersuchung ist es daher, dass in den Daten die Informationen über die Zielobjekte (Wunschsignal) gegenüber anderen aufgezeichneten Signalen überwiegen. Nach Möglichkeit werden dabei die Messeinstellungen so gewählt, dass dieses Verhältnis schon in den Rohdaten günstig ausfällt. Allerdings lassen sich gerade im urbanen Raum nie alle störenden Einflüsse vollkommen eliminieren. Daher werden die Datensätze üblicherweise mit verschiedenen Filtern bearbeitet, um die gewünschten Informationen im Anschluss an die Messungen (optimal) herauszuarbeiten. Zwar lassen sich sowohl die Bearbeitungs- als auch die Visualisierungsschritte von Georadardaten nicht generalisieren, als übliche Abfolge für die Nachbearbeitung kann aber folgende genannt werden:

1. Merge Regions (bei 3D-Datensätzen) Dadurch werden die mit verschiedenen Antennenanordnungen aufgenommenen Spuren miteinander verschnitten.

2. Zero Positioning. Laufzeitkorrektur, durch die die Radargramme jeweils mit der Reflexion der Bodenoberfläche beginnen.

3. Background Removal. Horizontaler Filter zur Betonung von Einzelobjekten.

4. (Kirchhoff-) Migration. Aufgezeichnete Objekte, die in den Radargrammen als Hyperbeln erscheinen, werden durch diesen Bearbeitungsschritt annäherend auf ihre tatsächliche Größe reduziert. Gleichzeitig ist eine Geschwindigkeitsbestimmung über die Breite der Hyperbeln möglich.

5. Hilbert Transformation kann sinnvoll sein, um die aufgezeichneten Amplituden als Beträge darzustellen.

Genauere Informationen zur Funktionsweise der beschriebenen Filtertechniken finden sich beispielsweise bei Olhoeft (2000).

\subsubsection{Visualisierung und Interpretation von Georadaten}

Neben den verschiedenen beschriebenen Bearbeitungsschritten zur Präparation der Zielinformation aus den aufgenommen Daten ist die Art der Darstellung entscheidend für eine gelungene Visualisierung des Untersuchungsgegenstands (in diesem Fall von Baumwurzeln) durch GPR-Messungen (Daniels 2000). Dazu gehört die Wahl eines geeigneten Amplituden- und Farbspektrums und insbesondere bei umfangreicheren Datensätzen die Auswahl des geeigneten Datenausschnitts. Bei dreidimensionalen Datensätzen muss zusätzlich auf die Wahl des geeigneten Laufzeitbereichs und des richtigen Blickwinkels geachtet werden (Daniels 2000, S. 11). 
Grundsätzlich ist zwischen der ein, zwei- und der dreidimensionalen Darstellung der Messergebnisse, welche auch von der Art der Datenerfassung abhängig ist, zu differenzieren (Abbildung 2.8. . Eindimensional meint die Präsentation einzelner Radarspuren (A-Scans; vgl. Abbildung $2.8 \mathrm{a}$ rechts), eine solche Spur wird auch als wavelet oder Wellenzug bezeichnet. Bei der entsprechenden Visualisierung wird die Signalamplitude zu den jeweiligen Laufzeiten dargestellt. Bei der zweidimensionalen Darstellungsform werden einzelne B-Scans analysiert. Eine übliche Darstellungsform ist ein Graustufen- oder Farbradargramm (vgl. Abbildung 2.8a links), bei dem die Amplituden der erfassten elektromagnetischen Wellen mit einem entsprechenden Farb- oder Grauwert dargestellt werden.

Im Untergrund liegende Objekte zeichnen sich typischerweise bei einer Datenaufzeichnung orthogonal zum Objektverlauf in zweidimensionalen Radargrammen (B-Scan) als Diffraktionshyperbeln ab (vgl. Abbildung 2.9). Diese Form kommt dadurch zustande, dass die in den Boden eingekoppelten elektromagnetischen Wellen sich nicht linear in den Untergrund ausbreiten, sondern annähernd kegelförmig abgestrahlt werden . Bei der Annäherung der Radarantenne an ein Objekt wird dieses deshalb schon erfasst, bevor die Antenne direkt über diesem positioniert ist (Abbildung 2.9 Position 1). Der Weg der ausgestrahlten Radarwellen von der Antenne zum Objekt kann in dem in Abbildung 2.9 dargestellten Schnitt als Hypotenuse in einem rechtwinkligen Dreieck angesehen werden, dessen drei Eckpunkte die Radarantenne an Position 1 und 2 sowie die Position des Objekts im Boden sind. Wenn die Radarantenne von Position 1 zu Position 2 bewegt wird, verkürzt sich die Hypotenuse (Laufzeit $\Delta t_{1}$ ) bis an Position 2 (Laufzeit $\Delta t_{2}$ ) kein Dreieck mehr besteht und die kürzeste Laufzeit zwischen Objekt und Radarantenne aufgezeichnet wird.

Im 2D-Radargramm wird die jeweilige Amplitude zur entsprechenden Laufzeit aufgezeichnet. In Abbildung 2.9 wird das Objekt, das sich an Position 2 befindet, daher schon an Position 1 mit der Laufzeit $\Delta t_{1}$ abgebildet. Durch die kontinuierliche Messung zwischen Position 1 und 2 bei kontinuierlich abnehmender Laufzeit zwischen Antenne und Objekt, ergibt sich im Radargramm eine Hyperbelform (2.9 schwarze Linie). Da die Laufzeiten zwischen Position 2 und Position 3 wieder zunehmen, ergibt sich der zweite Ast der Hyperbel.

Für die dreidimensionale Darstellung von Bodenradardaten gibt es verschiedene Varianten, die in Abbildung 2.8 dargestellt sind. Der aufgezeichnete Datensatz kann beispielsweise als Abfolge der aufgezeichneten Einzeltransekte in Form eines 2D-Radargramms dargestellt werden (Abbildung 2.8a). Dabei ähnelt die Datenanalyse der Auswertung von B-Scans, dient allerdings auch der Vorbereitung einer weiterführenden 3D-Darstellungsform, da sich hieraus so genannte Zeitscheiben/ time-slices beziehungsweise Horizontalschnitte erstellen lassen (Abbildung 2.8b). Bei diesen Zeitscheiben werden die Amplituden, die in einem angegebenen Zeitfenster liegen, in einen Horizontalschnitt projiziert. Für die Wahl des Zeitfensters werden ein Basiswert (z-Wert) und ein Laufzeitbereich (width) angegeben (dargestellt wird der Bereich $z \pm$ width/2).

Eine 3D-Darstellung ermöglicht es, den räumlichen Verlauf von aufgezeichneten Anomalien (Bereiche, in denen starke Amplituden vorliegen) zu verfolgen. Eine übliche Vorgehensweise bei der Analyse von 3D-Georadardatensätzen besteht darin, die Ergebnisse auf das Vorliegen von Anomalien zu untersuchen und deren räumlichen Verlauf mit dem wahrscheinlichen Verlauf und 


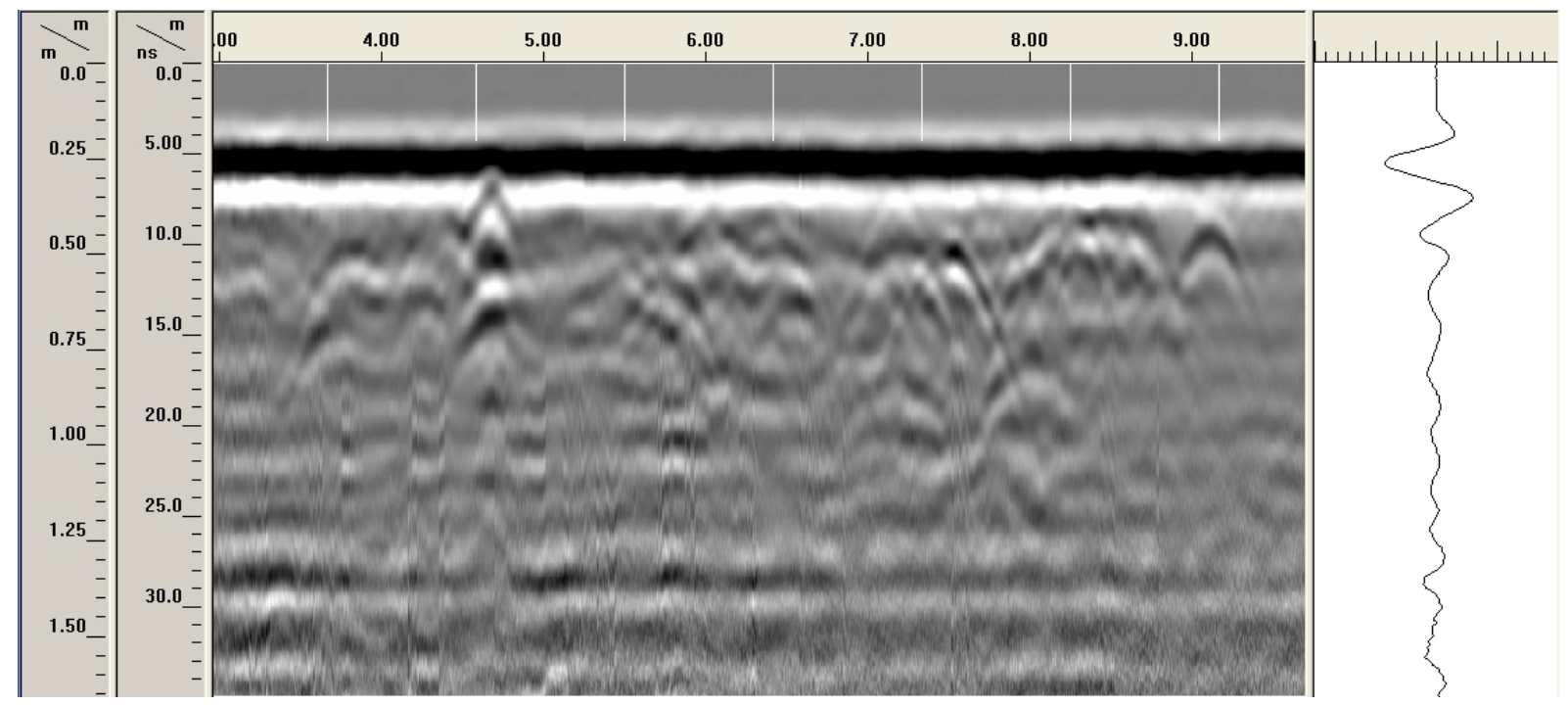

a) Der linke Bildteil zeigt einen B-Scan, der rechte den dazu gehörenden A-Scan an der Position $4.6 \mathrm{~m}$; links sind die Laufzeit und die anhand eines $\varepsilon_{\mathrm{r}}$-Wertes von 10 bestimmte Tiefe abgebildet; da es sich um unbearbeitete Rohdaten handelt, stimmt die Bodenoberfläche dabei noch nicht mit der 0 ns-Marke überein.
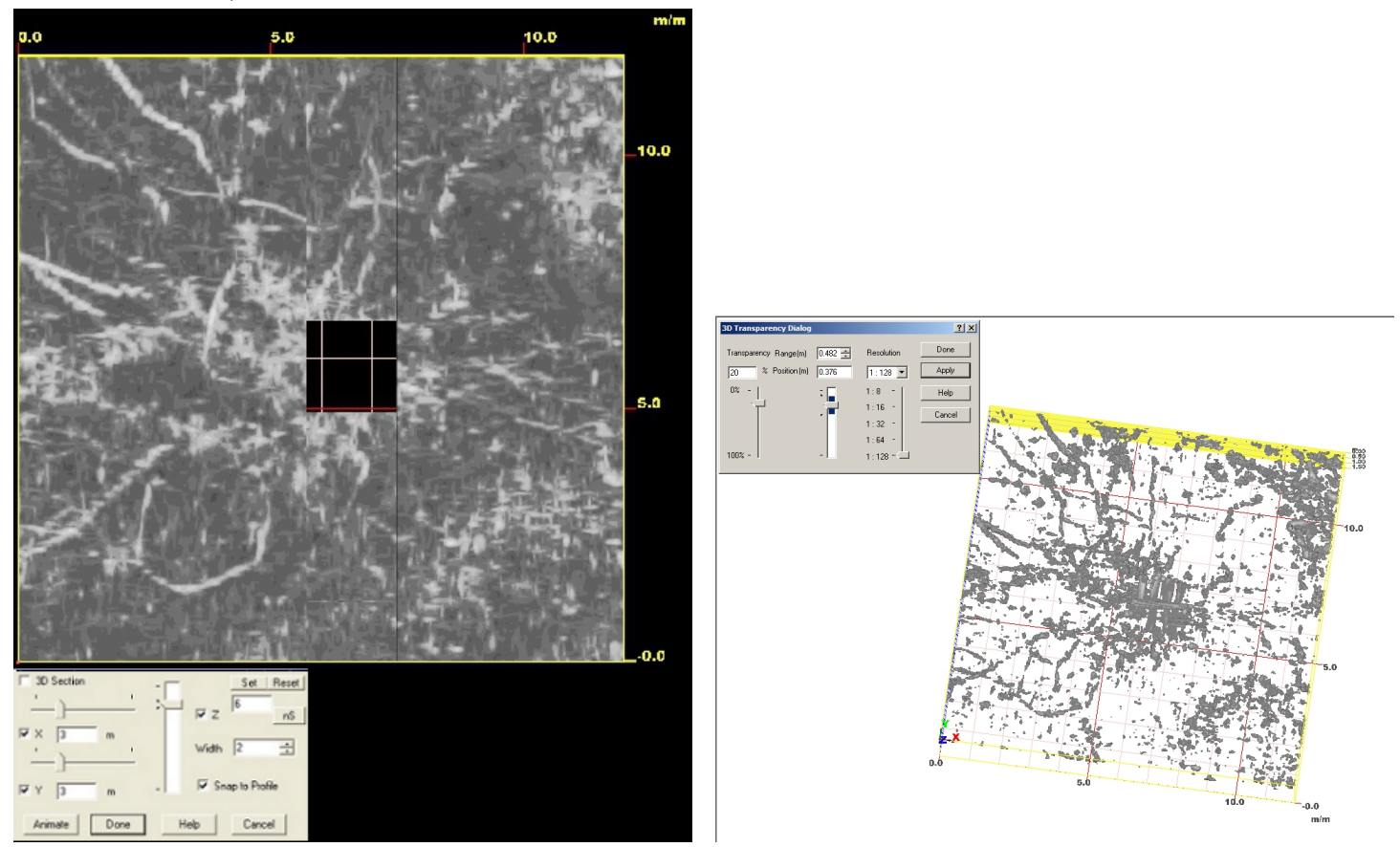

b) Visualisierung eines flächig aufgezeichneten Radar- c) 3D-Visualisierung des in 2.8b gezeigten Datensatdatensatzes (C-Scan) als Horizontalschnitt (Zeitscheibe). Bei dieser Darstellungsform kann nur ein Teil der zur Verfügung stehenden Daten visualisiert werden. zes als solid cube. Im Idealfall kann damit eine Freistellung des Zielobjekts im 3D-Modell erreicht werden.

Abbildung 2.8: Visualisierung eines Radardatensatzes als A- und B-Scan 2.8a und als C-Scan $2.8 \mathrm{~b}$ und $2.8 \mathrm{c}$. 
der wahrscheinlichen Lage von (Ziel-)Objekten im Boden abzugleichen. Im Fall von Leitungen kann dann beispielsweise der Lagebezug der Anomalie zu bekannten (oberirdisch erkennbaren) Objekten wie Gullies oder Straßenlaternen bei der Identifikation im Radargramm helfen. Im Fall von Baumwurzeln kann die Position des Stammes als Anhaltspunkt dienen.

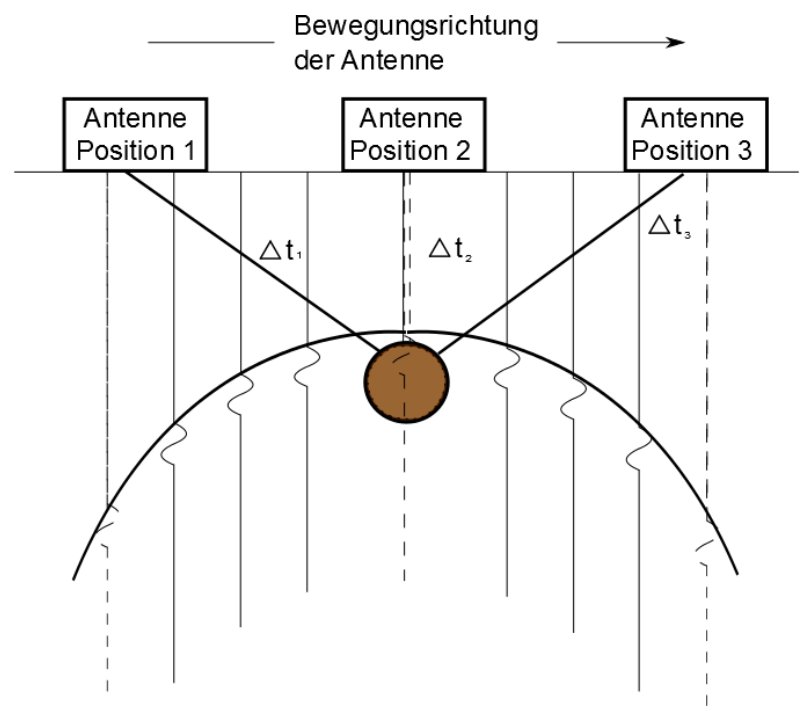

Abbildung 2.9: Entstehung einer Diffraktionshyperbel bei Bodenradarmessungen. Verändert nach Conyers (2004).

Eine weitere Darstellungsform, die sich aus räumlichen Datenaufzeichnungen erzeugen lässt, wird im Auswertungsprogramm RADAN 6.6 als solid cube bezeichnet. Bei dieser Visualisierungsform wird ebenfalls das Zeitfenster bzw. der Tiefenbereich angegeben, der betrachtet werden soll. Dazu kann ein Schwellenwert für die in diesem Bereich erfassten Amplituden angegeben werden. Es werden alle Datenpunkte abgebildet, deren Amplituden sich oberhalb dieses Wertes befinden $(100 \%=$ nur stärkste Amplitude; $0 \%=$ gesamtes aufgezeichnetes Amplitudenspektrum, vgl. Abbildung 2.8c). Hierdurch lässt es sich erreichen, dass Objekte bestimmter Amplitude im Raum annähernd freigestellt werden. Dabei ist zu beachten, dass auch unterschiedliche Objekte die gleiche Amplitude und gleiche Objekte unterschiedliche Amplituden aufweisen können. Deshalb gelingt die Präparation eines bestimmten Zielobjekts oder gleichartiger Zielobjekte durch diese Darstellung nicht zwangsläufig.

In einem 2D-Radargramm wird bei einem konstanten $\varepsilon_{r}$-Wert die relative Tiefenlage von zwei Objekten zueinander anhand der Laufzeiten ersichtlich, da das Objekt tiefer liegt, das zu einer späteren Laufzeit erfasst wird (Gleichung 2.2). Für die Bestimmung der absoluten Tiefenlage muss die Geschwindigkeit $v$ bzw. die Dielektrizitätskonstante $\varepsilon_{r}$ bekannt sein. Eine Bestimmung der Geschwindigkeit in einem Radargramm kann näherungsweise anhand der Steigung der Hyperbeläste vorgenommen werden (siehe hierzu z.B. Olhoeft 2000; Goodman et al. 2009). Steilere Hyperbeläste deuten dabei auf eine niedrige Ausbreitungsgeschwindigkeit hin, während bei einer höheren Ausbreitungsgeschwindigkeit flachere Hyperbeläste vorliegen (vgl. Abbildung 2.10 . 


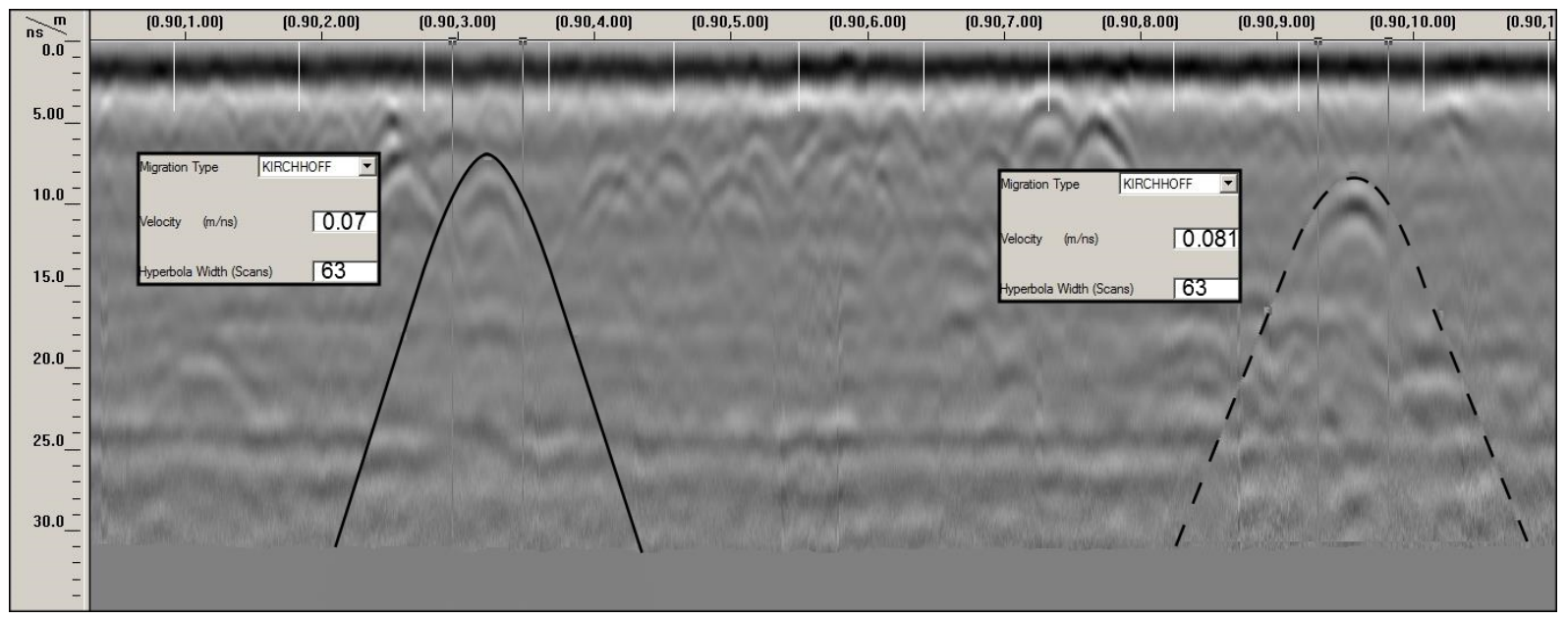

a) Ermittlung der Ausbreitungsgeschwindigkeit zweier Hyperbeln. Im Bereich der schmaleren Hyperbel (-) liegt eine Geschwindigkeit von $0,07 \mathrm{~m} \mathrm{~ns}^{-1}$ vor. Bei der breiteren Hyperbel (---) eine Geschwindigkeit von 0,081 $\mathrm{m} \mathrm{ns}^{-1}$.

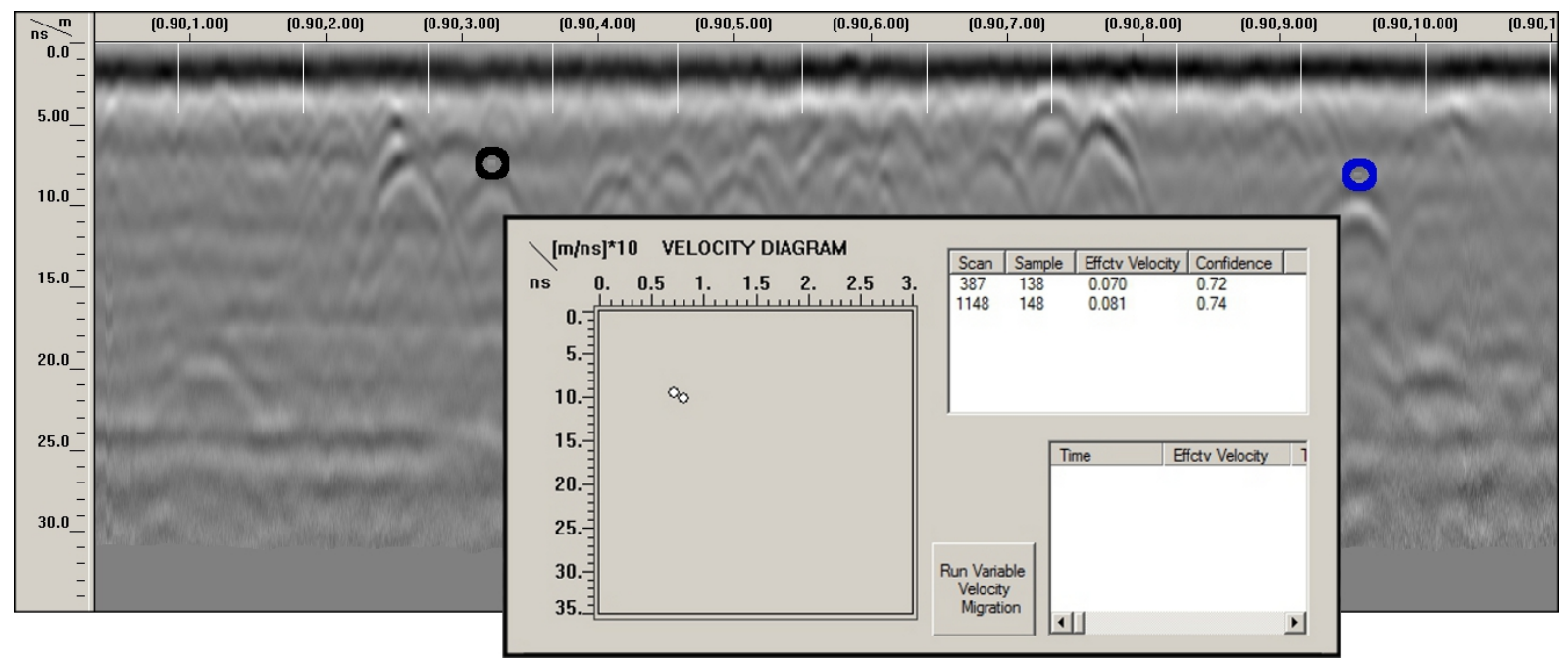

b) Im grauen Kasten werden die zu den einzelnen Laufzeiten erfassten Geschwindigkeiten abgebildet. Durch die differenzierte Erfassung der Geschwindigkeiten ist es möglich, bei heterogener Bodenzusammensetzung unterschiedliche Tiefenumrechnungen für verschiedene Laufzeitbereiche durchzuführen.

Abbildung 2.10: Geschwindigkeitsbestimmung über die Hyperbelbreite mit dem Programm RADAN 6.6. 


\subsubsection{Geoelektrik}

\section{Grundlegende Funktionsweise}

Der Teil der Geophysik, der sich mit der Sondierung und Kartierung von elektrischen Widerständen/ Leitfähigkeiten beschäftigt, ist die Geoelektrik oder auch elektrische Widerstandstomographie. Das Verfahren dient der Ermittlung des spezifischen elektrischen Widerstandes des Bodens (im folgenden wird der elektrische Widerstand vereinfachend teilweise nur als Widerstand bezeichnet), einer im Gegensatz zum elektrischen Widerstand von der Geometrie des Messobjekts unabhängigen Materialeigenschaft. Die Grundlage geoelektrischer Messungen ist das Ohmsche Gesetz (siehe hierzu z.B. Weidelt 2005 Zonge et al. 2005). Dieses beschreibt den Zusammenhang von Spannung (U in Volt) als Produkt des elektrischen Widerstands ( $\mathrm{R}$ in $\mathrm{Ohm}$ ) und der Stromstärke (I in Ampere):

$$
U=R I
$$

Der Kehrwert des Widerstands 1/R wird als elektrische Leitfähigkeit $\sigma$ bezeichnet und in der Einheit Siemens angegeben.

Bei elektrischen Widerstandsmessungen wird je nach Messfrequenz zwischen Gleichstrom- und Wechselstrommessungen unterschieden. Der elektrische Widerstand wird auch als Gleichstromwiderstand bezeichnet, da dieser von der Frequenz des zur Messung genutzten Stroms weitgehend unabhängig ist. Bei Wechselströmen muss neben dem Gleichstromwiderstand auch der frequenzabhängige Teil des Widerstands berücksichtigt werden, der mit dem Gleichstromwiderstand als so genannte Impedanz zusammengefasst wird.

Die praktische Umsetzung von Geoelektrikmessungen (Abbildung 2.11) erfolgt, indem ein niederfrequenter Wechselstrom in den Boden eingespeist wird. Dessen Frequenz wird dabei so niedrig gewählt, dass der frequenzabhängige Anteil bei der eigentlich erfassten Impedanz (dem frequenzabhängigen Widerstand) so gering ausfällt, dass dieser vernachlässigt werden und der gemessene Wert mit dem frequenzunabhängigen Gleichstromwiderstand (auch: Ohmscher Widerstand) gleichgesetzt werden kann. Trotz des zu vernachlässigenden Effekts auf die Messgröße wirkt sich der niederfrequente Wechsel in der Stromflussrichtung bei den Messungen entscheidend aus, da hierdurch keine störenden Polarisierungseffekte an den eingesetzten Elektroden auftreten.

Zur Ermittlung des elektrischen Widerstands wird in der Regel eine Vier-Punkt-Messung durchgeführt, bei der an zwei Punkten Strom eingespeist wird und an zwei anderen Messpunkten eine Spannungsdifferenz ermittelt wird. Dies geschieht, um den Einfluss der unbekannten Übergangswiderstände auf den zu messenden Bodenwiderstand auszuschalten. Übergangswiderstände liegen bei allen Übergängen von elektrischem Strom von einem in einen anderen Körper vor.

Um bei einer Geoelektrikmessung nicht nur den geometrieabhängigen elektrischen Widerstand zu ermitteln, sondern den spezifischen Widerstand als die gesuchte Materialeigenschaft, muss zusätzlich ein Geometrie- oder auch Konfigurationsfaktor G (Gleichung 2.10) berücksichtigt werden, der bei verschiedenen geoelektrischen Messanordnungen unterschiedlich ist. Im Fall eines homogenen Untergrundaufbaus kann dann unter Berücksichtigung des Konfigurationsfaktors der spezifische elektrische Widerstand bestimmt werden. Da die genauen Bodenverhältnisse in der Regel inhomogen sind, wird mit einer geoelektrischen Messung unter Berücksichtigung des 
Konfigurationsfaktors der scheinbare spezifische Widerstand $\rho_{a}$ bestimmt.

Für den scheinbaren spezifischen Widerstand $\rho_{a}$ gilt entsprechend:

$$
\rho_{a}=G \cdot \frac{\Delta U}{I}
$$

Typische geoelektrische Messanordnungen sind die Dipol-Dipol-, Schlumberger- und WennerAnordnung (vgl. Abb. 2.11).

Die jeweiligen Messanordnungen unterscheiden sich hinsichtlich ihrer Auflösungseigenschaften. Während beispielsweise mit Dipol-Dipol-Auslagen eine hohe horizontale Auflösung erreicht wird, eignen sich Schlumberger-Messungen zur vertikalen Sondierung über einem fixen Messpunkt. Zu diesem Zweck eignen sich Wenner-Anordnungen nicht, da hierbei alle Elektroden versetzt werden, wodurch die Untersuchungstiefe nicht zunimmt. Aufgrund des gleichbleibenden Elektrodenabstands bleibt die erreichte Sondierungstiefe gering (Oldenburg und Li 2005).

Der Abstand zwischen den Elektroden (spacing) gibt bei den Messungen die horizontale Auflösung vor. Im Regelfall werden bei der Dipol-Dipol-Anordnung benachbarte Elektrodenpaare für die einzelnen Dipole gewählt. Die Gesamtauslagenlänge ist entscheidend für die maximale Eindringtiefe des eingespeisten Stroms. Je nach gewählter Messanordnung können Tiefenbereiche zwischen 15 bis $20 \%$ der Auslagenlänge (Wenner) bis hin zu $50 \%$ (Schlumberger) erreicht werden.

Wenn der Geometriefaktor bekannt ist (was in der Regel der Fall ist), kann der scheinbare spezifische elektrische Widerstand, als Materialeigenschaft nach Gleichung 2.10, durch eine geoelektrische Messung ermittelt werden. Nach Just et al. (1997) ist die „Voraussetzung dabei [...], dass sich die Messwerte als Projektion der Parameterverteilung darstellen lassen." (Just et al. 1997, S. 3). Um dann schließlich von dieser Projektion auf den eigentlich gesuchten spezifischen Widerstand $\rho$ schließen zu können, wird eine Inversionsrechnung („dh. die Umkehrung der Projektion" Just et al. 1997, S. 3) durchgeführt. Dadurch können aus den am Rand (der Bodenoberfläche) gemessenen Spannungsdifferenzen die Leitfähigkeitsverteilungen im Untergrund hochaufgelöst und differenziert rekonstruiert und betrachtet werden. Dabei ist zu beachten, dass die Ergebnisse von Inversionsrechnungen nicht eindeutig sind. Stattdessen gibt es immer eine Vielzahl möglicher Kombinationen von Schichtmächtigkeiten und spezifischen Widerständen, die die gemessenen Werte plausibel erklären.

Grundlegende Informationen zum inversen Problem in der Geophysik finden sich bei Oldenburg und $\operatorname{Li}(2005)$.

\subsubsection{Visualisierungsmöglichkeiten von Geoelektrikdaten}

Für die Darstellung von Geoelektrikdaten gilt Ähnliches wie für die in Kapitel 2.3.2.4 beschriebene Darstellung der Geordardaten. Es kann eine eindimensionale Darstellung erfolgen, bei der der spezifische Widerstand an einem Punkt in Abhängigkeit der Bodentiefe dargestellt wird oder es können 2D- und 3D-Tomogramme erstellt werden, bei denen die Widerstandsverteilung entlang eines Transekts oder auf einer Fläche mit einer Farbabstufung dargestellt sind (Abbildung 2.12). 


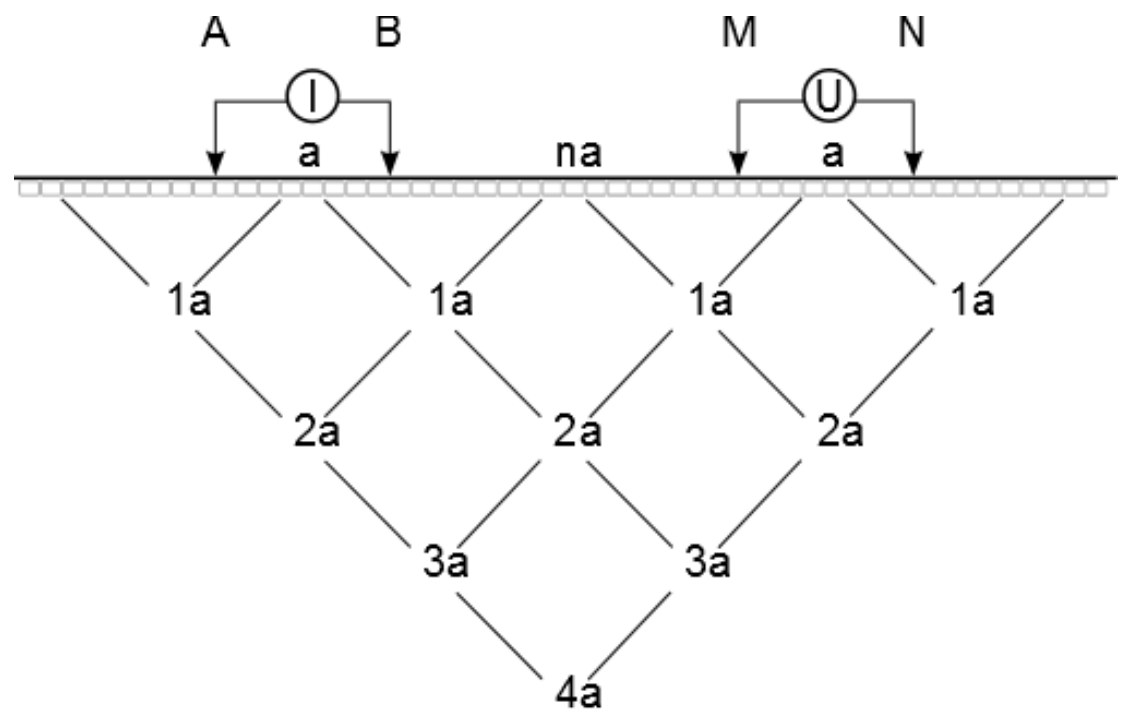

a) Dipol-Dipol-Anordnung: Entlang eines Messtransekts werden sowohl die Einspeise- $(\mathrm{AB})$ als auch die Messelektroden (MN) verschoben. Der Abstand der beiden Elektroden, die so genannte Dipollänge $a$, bleibt dabei gleich, während die Abstände der beiden Elektrodenpaare jeweils um ein Vielfaches der Dipollänge $(n a)$ variieren. Durch die Vergrößerung dieses Abstands werden größere Tiefenstufen (level) erfasst.

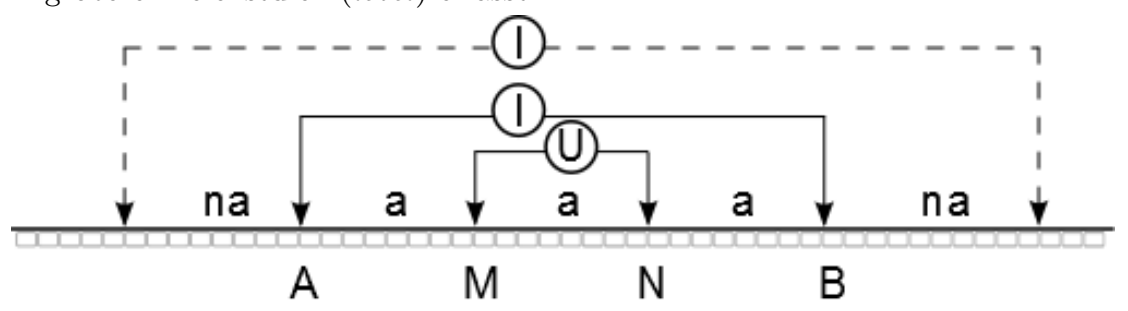

b) Schlumberger-Anordnung: Während die innen angeordneten Messelektroden $(\mathrm{MN})$ mit festem Abstand ( $a$ ) an einer Stelle verbleiben, wird der Abstand der außen liegenden Messelektroden um ein Vielfaches von a vergrößert. Dadurch wird eine zunehmende Tiefensondierung an einem Punkt erreicht.

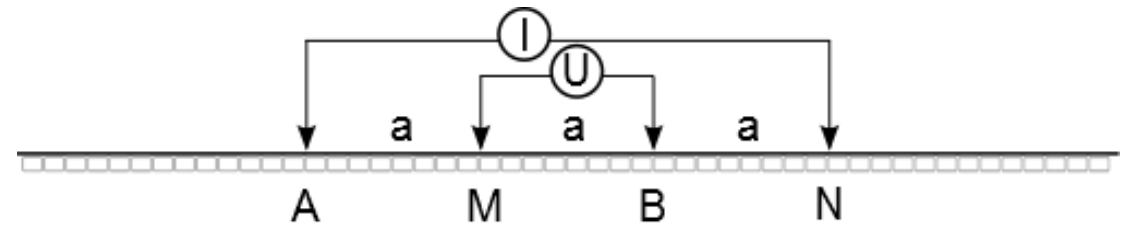

c) Wenner-Anordnung: Spezialfall der Schlumberger-Anordnung, bei dem der Abstand aller Elektroden konstant ist.

Abbildung 2.11: Verschiedene Geoelektrikanordnungen- verändert nach Zonge et al. (2005, S. 271,274 f.). 


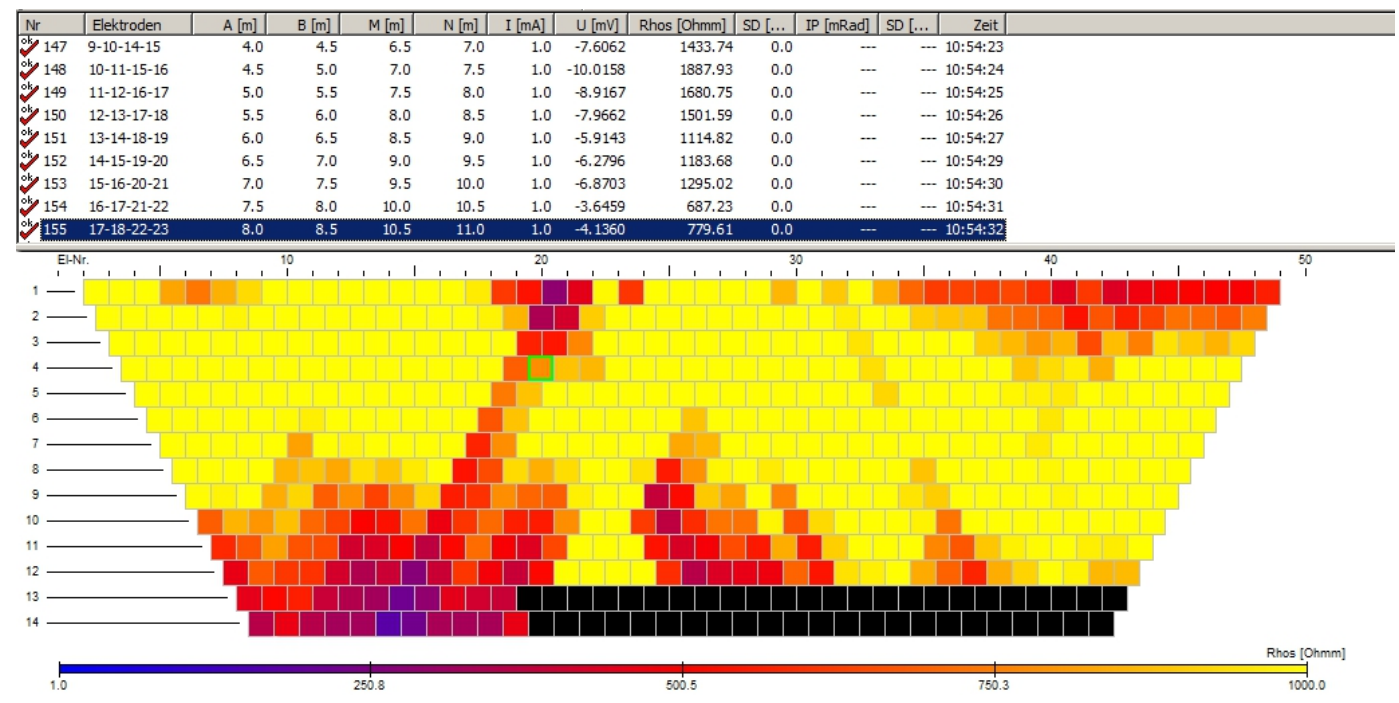

a) Datenerfassung bei der Messung. Durch die zunehmende Vergrößerung des Abstands zwischen den beiden Dipolen werden größere Sondierungstiefen erreicht. Blau unterlegt ist die Erfassung von Messwert 155 ( $\square$ im unteren Bildteil).

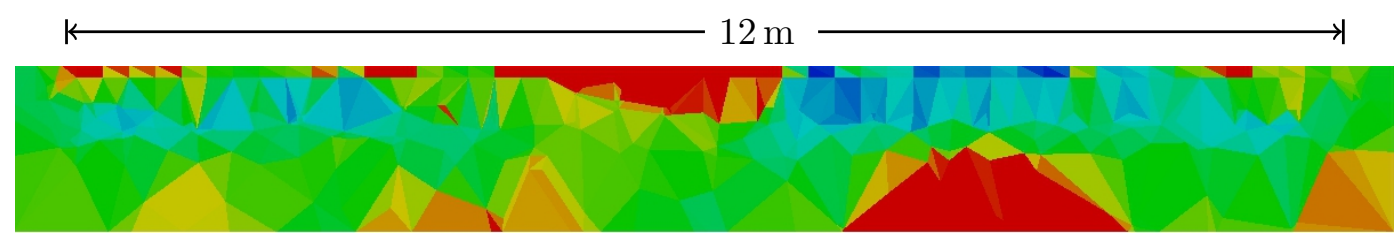

b) Durch Inversion eines einzelnen Transekts erstelltes 2DWiderstandstomogramm.
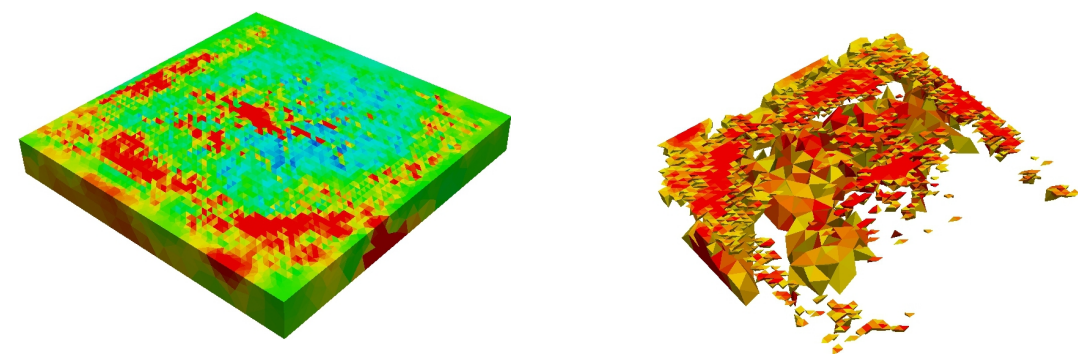

c) Durch Inversion der durch mehrere Transekte erfassten Widerstandsdaten erstelltes 3D-Widerstandstomogramm. Im linken Bild wird das gesamte erfasste Widerstandsspektrum dargestellt; im rechten Bild werden nur die Bereiche, in denen der spezifische Widerstand über einem bestimmten Schwellenwert liegt, dargestellt.

Abbildung 2.12: Datenerfassung und Visualisierung bei der elektrischen Widerstandstomographie. Im ersten Schritt werden die Daten aufgezeichnet. Anschließend erfolgt je nach Umfang der Messung eine zwei oder dreidimenionale Inversion. 


\subsection{Elektromagnetische Eigenschaften von Böden und Wurzeln}

\subsubsection{Dielektrische Permittivität $\varepsilon_{\mathrm{r}}$}

Die im Zusammenhang mit den Bodenradarmessungen bereits eingeführte dielektrische Permittivität, Dielektrizitätszahl oder auch Dielektrizitätskonstante $\varepsilon_{\mathrm{r}}$ eines Mediums (vgl. Kapitel 2.3.2 gibt an, wie gut das Medium einem angelegten elektromagnetischen Wechselfeld durch Ladungstrennung entgegenwirken kann. Für GPR-Messungen ist dabei besonders wichtig, dass $\varepsilon_{\mathrm{r}}$ wesentlich vom Wassergehalt des Bodens beeinflusst wird. Insgesamt reicht das Spektrum von natürlichen Dielektrizitätswerten von 1 für Luft (genau genommen Vakuum) bis 81 für reines Wasser. Böden setzen sich dabei aus verschiedenen Komponenten zusammen, die unterschiedliche Dielektrizitätskonstanten aufweisen. Die daraus resultierende, effektive Dielektrizitätskonstante ergibt sich nach Alteköster (2004, S. 17) dabei aus:

1. den Volumenanteilen und Dielektrizitätszahlen der einzelnen Komponenten

2. der geometrischen Form und Verteilung der Komponenten

3. der physikalischen und chemischen Wechselwirkungen zwischen den Komponenten

Die einzelnen Bestandteile im Boden sind dabei freies und gebundenes Wasser, Luft und die Bodenmatrix mit ihren verschiedenen Bestandteilen (Weidelt 2005).

Der empirisch ermittelte Zusammenhang zwischen dem volumetrischen Bodenwassergehalt $\Theta_{v}$ und der Dielektrizitätszahl ist nach Topp et al. (1980):

$$
\varepsilon_{r, b}=3,03+9,3 \Theta_{v}+146,0 \Theta_{v}^{2}-76,7 \Theta_{v}^{3}
$$

Tabelle A.1 im Anhang gibt einen Überblick zu den Dielektrizitätszahlen verschiedener Materialien.

\subsubsection{Elektrische Leitfähigkeit $\sigma$}

Nach Weidelt (2005) ist bei niederfrequenten elektromagnetischen Untersuchungsverfahren wie der elektrischen Widerstandstomographie die elektrische Leitfähigkeit die wichtigste Eigenschaft eines Mediums, die die Ausbreitung der erzeugten elektromagnetischen Felder bestimmt.

Die elektrische Leitfähigkeit wird in die elektronische Matrixleitfähigkeit, die elektrolytische Leitfähigkeit und die Grenzflächenleitfähigkeit unterteilt. Erstere bezeichnet dabei die Leitfähigkeit der Gesteine und Substrate selbst und wird durch die in diesen vorhandenen freien Elektronen verursacht. Die elektrolytische Leitfähigkeit ist dagegen die Stromleitung durch wässrige Lösungen im Porenraum, die eine starke Erhöhung der Leitfähigkeit bewirkt. Die Grenzoder Überschussleitfähigkeit ist der verbleibende Teil der Gesamtleitfähigkeit, der nicht durch die beiden anderen Leitfähigkeitsmechanismen erklärt werden kann. Diese wird im Wesentlichen durch das Kationenaustauschvermögen von Tonen verursacht (Weidelt 2005).

Tabelle A.2 im Anhang zeigt eine Übersicht zu den spezifischen Widerständen verschiedener Materialien. 


\subsection{Vorarbeiten zur Wurzeldetektion mit geophysikalischen Methoden}

\subsubsection{Wurzeldetektion mit Georadar}

Die Arbeiten verschiedener Autoren zeigen auf, dass es grundsätzlich möglich ist, durch den Einsatz des Georadars Baumwurzeln zu detektieren.

Erste Untersuchungen zu dieser Thematik haben Hruska et al. (1999) durchgeführt. Die Autoren kartierten flächenhaft mit einer $450 \mathrm{MHz}$-Bodenradarantenne die Wurzeln von Waldbäumen bis zu einem Minmaldurchmesser von $3 \mathrm{~cm}-4 \mathrm{~cm}$. Weitere Arbeiten, in denen flächenhafte Wurzelkartierungen mittels GPR vorgestellt werden, sind die von Van Vleck (1999), Cermak et al. (2000), Lorra und Kathage (2004), Hagrey und Meissner (2004), Hagrey (2007), Zenone et al. (2008) und Leucci (2011). Dabei werden Antennen mit Mittenfrequenzen zwischen $1500 \mathrm{MHz}$ (Van Vleck 1999) und $400 \mathrm{MHz}$ (Lorra und Kathage 2004), Rasterabstände zwischen $2 \mathrm{~cm}$ (Van Vleck 1999) und $50 \mathrm{~cm}$ (Lorra und Kathage 2004) sowie verschiedene Antennenkonfigurationen und -anordnungen eingesetzt. Bis auf Hagrey und Meissner (2004) (CMP-Messungen) und Butnor et al. (2006) (Bohrlochgeoradar) kommen dabei Common-Offset-Messungen zum Einsatz. Bei den Antennenorientierungen werden teilweise orthogonal zueinander liegende Raster gewählt, teilweise aber auch nur in eine Richtung verlaufende Parallelprofile aufgenommen.

Bei allen erwähnten Untersuchungen zeichnen sich die Wurzeln als baumnahe Anomalien ab. Als Grenzen für den Einsatz der Messmethoden werden von Cermak et al. (2000) tonige und schuttbelastete Böden genannt, Lorra und Kathage (2004) betonen, dass vertikal verlaufende Wurzeln mittels Georadar nicht zu erfassen sind.

Neben diesen dreidimensionalen Untersuchungen stellen weitere Arbeiten zweidimensionale Messungen vor, die folglich die Untergrundverhältnisse entlang einzelner Profile zeigen. Ziel dieser Arbeiten ist die Ermittlung grundlegender Auflösungseigenschaften von Baumwurzeln mittels Georadar. In einer der ersten Arbeiten dazu untersuchten Butnor et al. (2001) die Unterschiede der Wurzelortung mit dem Bodenradar auf verschiedenen Standorten, wobei auch Antennen verschiedener Mittenfrequenzen eingesetzt wurden. Dabei erweisen sich hohe Ton- und Wassergehalte als störend, während die besten Ergebnisse auf trockenen Sandböden erzielt werden. Ein wesentlicher Aspekt der Arbeit von Butnor et al. (2001) ist die Ermittlung des Wurzeldurchmessers aus den aufgezeichneten Radardaten durch statistische Analysen. Die Autoren ermitteln eine hohe Korrelation zwischen dem Wurzeldurchmesser und der Amplitude der aufgezeichneten Reflexion.

Ein ähnliches Untersuchungsziel hat die Arbeit von Butnor et al. (2003b), bei der es um die Ermittlung der in den Wurzeln enthaltenen Biomasse aus den Radardaten geht.

Die Ermittlung des Wurzeldurchmessers aus den Messdaten ist auch Gegenstand der Arbeiten von Dannoura et al. (2008) und Hirano et al. (2008).

Der Autor der vorliegenden Arbeit hat Zwischenergebnisse der Forschungsarbeit, auf der diese Dissertation basiert in verschiedenen Tagungsbänden publiziert (vgl. Vianden et al. 2009, Vianden 2009, Weihs et al. 2010b; Weihs et al. 2010a; Vianden et al. 2010; Vianden et al. 2011; Vianden et al. 2012). 


\subsubsection{Wurzeldetektion mit Geoelektrik}

Die ersten Studien zur Wurzeldetektion mit der elektrischen Widerstandstomographie erfolgen in Verbindung mit Messungen durch andere geophysikalische Untersuchungsmethoden. Als erste Arbeit ist die von Hagrey und Meissner (2004) zu nennen. Die Autoren sind in der Lage, aufgrund unterschiedlicher Widerstandswerte zwischen Fein- und Grobwurzeln zu unterscheiden. Eine weitere Veröffentlichung (Hagrey 2007) zeigt die Möglichkeit auf, mittels geoelektrischer Langzeitmessungen die Wasseraufnahme von Bäumen anhand der Widerstandsänderungen im Boden nachvollziehen zu können.

In der Untersuchung von Amato et al. (2008) wird aus der hohen Korrelation zwischen den ermittelten Widerstandswerten und der Wurzelmasse, die Möglichkeit abgeleitet, die Wurzelmassenverteilung an einem Baumstandort durch geoelektrische Untersuchungen quantifizieren zu können. Während die Untersuchungen von Amato et al. (2008) dabei auf ein einzelnes Transekt beschränkt bleiben, untersuchen Rossi et al. (2010) flächenhaft die Widerstandsverteilung im Bereich einer Plantage und ermitteln ebenfalls eine hohe Korrelation zwischen den Widerstandswerten und der Wurzelverteilung im Untergrund.

Leucci (2011) setzt neben den eingangs erwähnten Radarmessungen im Rahmen seiner Untersuchungen auch die Geoelektrik ein. Er kommt zu dem Ergebnis, dass sich die Wurzelbereiche im untersuchten Baumbestand als hochohmige Anomalien von der Umgebung abheben. Zusätzlich setzt dieser Autor im Rahmen seiner Untersuchung auch seismische Messungen ein. Neben diesen Untersuchungen ist die Dissertation von Lazzari (2008) zu erwähnen, die in ihrer Arbeit die Auswirkung unterschiedlicher Elektrodenkonfigurationen auf die Wurzeldetektion mittels elektrischer Widerstandstomographie untersucht hat.

Tabelle 2.1 zeigt eine Übersicht zu den Arbeiten anderer Arbeitsgruppen. 


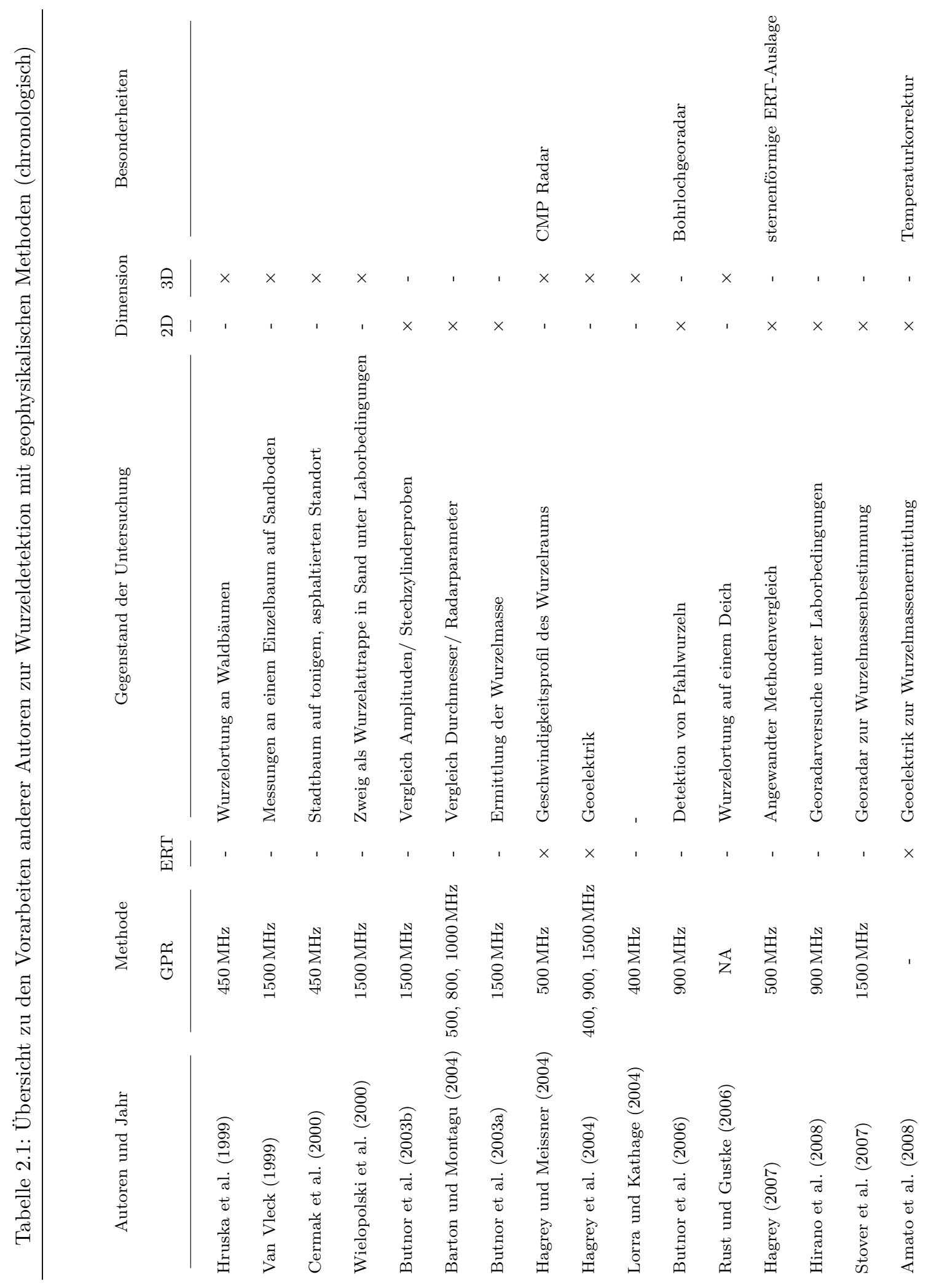




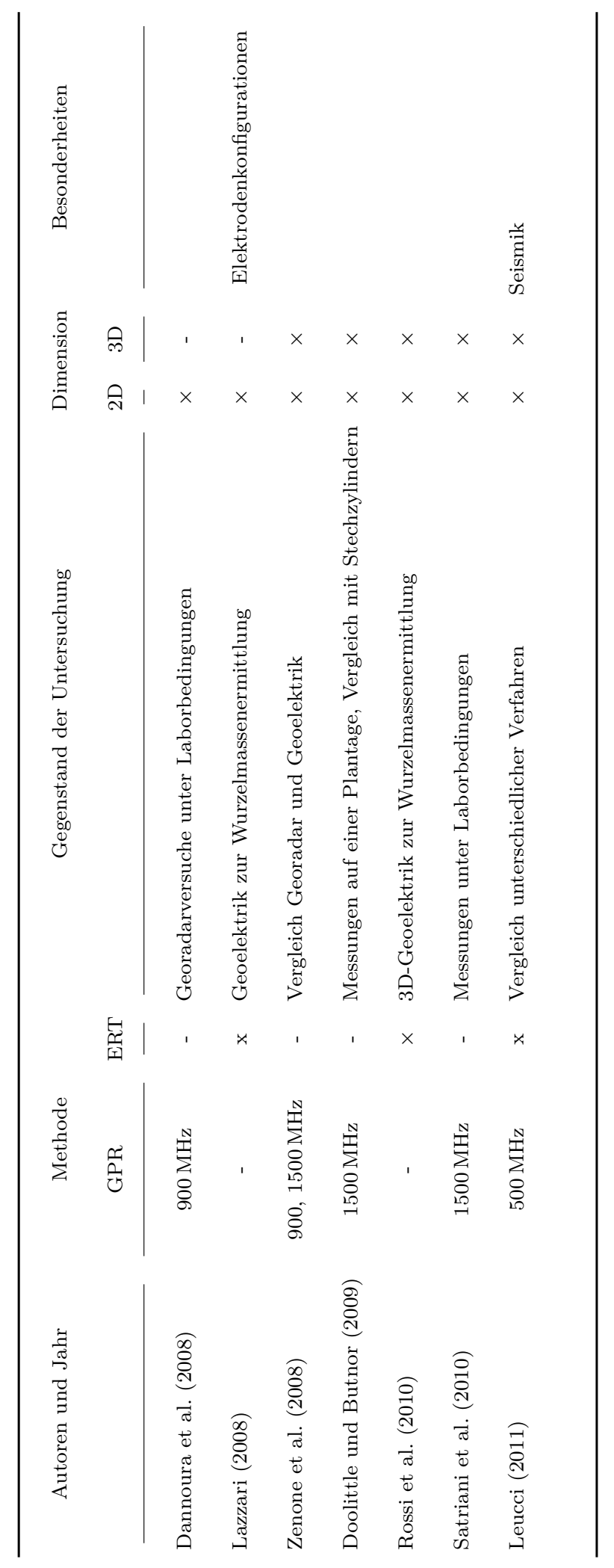





\section{Material und Methoden}

\subsection{Untersuchungsdesign}

Wie aus den Ausführungen in Kapitel 2 (Tabelle 2.1) ersichtlich wird, wurden die meisten bisher erfolgten Untersuchungen zur Wurzeldetektion mit geophysikalischen Methoden unter kontrollierten Bedingungen (Butnor et al. 2001; Butnor et al. 2003b Barton und Montagu 2004) durchgeführt. Zudem sind bei diesen Studien in der Regel nur einzelne Transekte untersucht worden (Hirano et al. 2008, Dannoura et al. 2008).

Dagegen zeigen bisher nur wenige dreidimensionale Untersuchungen an unveränderten Baumstandorten auf, welche Ergebnisse mit den Verfahren unter realistischen Verhältnissen erzielt werden können. Bis auf zwei Ausnahmen (Cermak et al. 2000 Leucci 2011) fanden diese Messungen zudem nicht auf städtischen Standorten statt, sondern konzentrieren sich auf Forst- und Waldgebiete. Daher liegen bislang nur geringe Kenntnisse über das Anwendungspotential der Methoden zur Wurzelortung auf urbanen Standorten vor.

Diese Lücke soll durch die vorliegende Arbeit geschlossen werden. Ein Untersuchungsziel ist es, zu zeigen, welche Möglichkeiten bei der Visualisierung bestehen und darüber hinaus eine Verbesserung der Auflösung im Vergleich zu den genannten, bisherigen Untersuchungen zu erreichen.

Ein weiterer Leitgedanke bei den Untersuchungen ist es, dass gerade unter schwierigen Bedingungen eine Interpretationshilfe durch die flächenhafte Kartierung eines Baumumfeldes gegeben sein sollte. Denn dadurch lassen sich die an einem Untersuchungsstandort erfassten Objekte anhand ihrer Topologie unterscheiden, während bei der Ansprache in zweidimensionalen Radargrammen Interpretationsschwierigkeiten bestehen können. Dies gilt insbesondere für städtische Standorte.

Gleichzeitig ist bekannt, dass abhängig von den Beschaffenheiten eines Standorts Grenzen bei der Visualisierung und Identifikation von Wurzeln durch geophysikalische Methoden bestehen. Die Morphologie des Wurzelsystems und die vorherrschenden Bodeneigenschaften sind die wesentlichen Einflussfaktoren bei der Wurzeldetektion mit geophysikalischen Methoden. In der bestehenden Literatur wird diese Limitierung aber nur als Randbemerkung genannt. Anhand von ausgewählten Untersuchungsbeispielen sollen die Grenzen der Wurzelortung mit den eingesetzten geophysikalischen Methoden als Teil der vorliegenden Arbeit verdeutlicht werden.

Das Untersuchungsdesign, also der Ablauf der durchgeführten Untersuchungen, ist in Abbildung 3.1 dargestellt.

Die Messungen folgen dem Schema, von kontrollierten, eigens eingerichteten Versuchen über annähernd ungestörte städtische Standorte an urbane Extremstandorte (z.B. Straßenränder) heranzutreten, um somit ein möglichst breites Spektrum urbaner Standorte zu erfassen. Die drei Untersuchungsschwerpunkte, die sich daraus ergeben, bauen dabei aufeinander auf. So werden im 
Rahmen der Untersuchungen unter kontrollierten Bedingungen die grundlegenden Auflösungs- und Abbildungsmöglichkeiten untersucht (Schwerpunkt 1 Abbildung 3.1). In Schwerpunkt 2 werden die wesentlichen Parameter für die dreidimensionalen Messungen ermittelt. Die Aufnahmen auf diesen Flächen werden dabei auch mit zum Ergebnis für Schwerpunkt 3, den Messungen auf unterschiedlichen urbanen Standorten, hinzugezogen.

Das Georadar bildet den Kern der Untersuchungen, weil es die Methode mit der höheren Auflösung ist. Die Geoelektrik wird benutzt, um ergänzende Daten zur Interpretation der Georadarmessungen zu erhalten. Daher wurden zu diesem Verfahren keine Vorversuche durchgeführt und keine grundlegenden Fragestellungen bearbeitet. Die Messungen dienen stattdessen als Interpretationshilfe für die Georadarmessungen und werden besonders in Situationen benötigt, in denen keine Informationen aus Aufgrabungen oder Beprobungen vorliegen. 


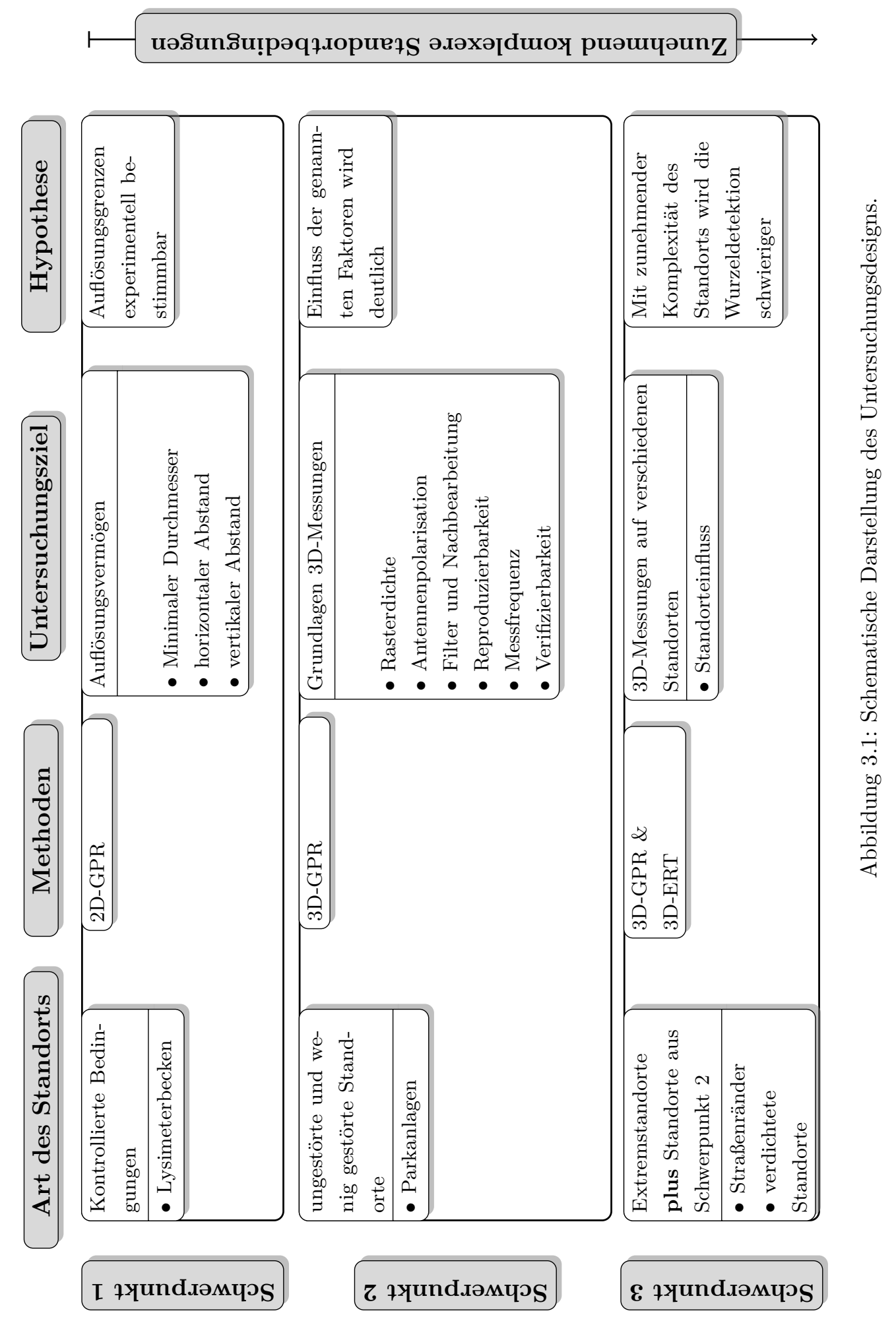




\subsubsection{D-Georadarmessungen in den Versuchsbecken}

Hintergrund der Messungen in den Versuchsbecken ist die Ermittlung des vertikalen und horizontalen Auflösungsvermögens des zur Verfügung stehenden Georadarsystems. Die Untersuchungen umfassen die Bestimmung des Durchmessers, bis zu dem eine Ortung von Wurzeln möglich ist, sowie die vertikalen und horizontalen Abstände, die zwischen Wurzeln liegen müssen, um diese voneinander unterscheiden zu können.

Daneben wird in einer weiteren Versuchsanordnung eine Vergleichsmessung mit Wurzeln und anderen Objekten, die in urbanen Böden zu erwarten sind (Kabel, Rohre, Hohlräume, etc.) durchgeführt.

\subsubsection{D-Messungen zu grundlegenden Fragestellungen}

Ein wesentlicher Einflussfaktor auf die mittels Georadar erzielbaren Ergebnisse ist die Antennenpolarisation. So wirkt sich neben der Antennenbauart (z.B. Horn- oder Dipolantenne) die Anordnung der Antenne zur Messspur und zum Untersuchungsobjekt entscheidend auf das Messergebnis aus (vgl. Annan (2005, S. 414 f.)).

Ein weiterer Untersuchungspunkt ist die Ermittlung des Messrasters, mit dem die Visualisierung der Wurzeln in einem praktikablen Zeitrahmen bestmöglich gelingt. Hier wird untersucht, inwiefern durch die Verdichtung des Messrasters an einem Untersuchungsstandort die Aussagekraft und die Auflösung des Messergebnisses gesteigert werden kann. Dieser Aspekt ist sowohl für die praktische Anwendung als auch für umfangreiche wissenschaftliche Messungen relevant. Die Relevanz ergibt sich daraus, dass in beiden Fällen ein Kompromiss zwischen Zeitbedarf und Datenqualität getroffen werden muss.

Für die praktische Nutzung des Georadars zur Wurzeldetektion ist es wichtig, die Reproduzierbarkeit der Ergebnisse beurteilen zu können. Nur dadurch ergibt sich beispielsweise die Möglichkeit, Bodenradarmessungen zum Wurzelschutz auf Baustellen einzusetzen und den Zustand des Wurzelsystems im Anschluss an die Baumaßnahme mit der im Vorfeld aufgenommenen Situation vergleichen zu können. Auch der Einsatz des Georadars für ein mögliches Langzeitmonitoring der Entwicklung von Wurzelsystemen ist an eine erfolgreiche Reproduzierbarkeit gebunden.

Im Kapitel 2.3.2.3 wurde erläutert, dass zur Visualisierung der Messergebnisse verschiedene Filter und Nachbearbeitungsschritte eingesetzt werden. In diesem Abschnitt wird untersucht, wie sich die einzelnen Bearbeitungsschritte auf die Ergebnisdarstellung auswirken.

Die Auflösung des Georadarverfahrens hängt maßgeblich von der Arbeitsfrequenz (genauer: der Mittenfrequenz der eingesetzten Antenne) ab (vgl. Kapitel 2). Da für die in dieser Arbeit durchgeführten und präsentierten Messungen im wesentlichen nur eine Antenne zur Verfügung stand, kann der Einfluss der Messfrequenz nur anhand von einer einzelnen Vergleichsmessung mit einer höherfrequenten Antenne auf der Messfläche Schwarzkiefer (Tabelle 3.1 Baum 2a) untersucht werden.

Da die Messungen, besonders in sensiblen Bereichen wie städtischen Parkanlagen nicht in jedem Fall durch den Vergleich mit Freilegungen oder Stechzylinderbeprobungen verifiziert werden 
können, muss eingangs untersucht werden, inwiefern die Ansprache der in den Messergebnissen dargestellten Anomalien den realen Bodenverhältnissen entspricht. Die Verifikation erfolgt mit verschiedenen Methoden (vgl. Kapitel 3.5).

\subsubsection{D-Messungen auf unterschiedlichen Standorten}

Bei den 3D-Messungen an ausgewählten Standorten geht es darum, die Möglichkeiten und Grenzen der Verfahren an städtischen Baumstandorten zu zeigen, was aufgrund der Vielfalt möglicher Kombinationen von Wurzelsystemen und Untergrundaufbauten nur exemplarisch durchgeführt werden kann. Die Auswahl der hier gezeigten Messbeispiele entspricht dem in Kapitel 3.1 erläuterten Schema. Die Durchführung der Messungen und die Ergebnisdarstellung orientiert sich an den im Rahmen der grundlegenden 2D- und 3D-Messungen erarbeiteten Vorgaben.

\subsection{Untersuchungsstandorte}

\subsubsection{Auswahl der Untersuchungsstandorte}

Für die Ermittlung der grundlegenden Auflösungseigenschaften des Georadars (Abbildung 3.1 Schwerpunkt 1) wurde die Lysimeterbeckenanlage im Neuen Botanischen Garten in Göttingen gewählt, in der Versuchsanordnungen gezielt umgesetzt worden sind.

Wie in Kapitel 2 erläutert, kann weder anhand der Baumart noch anhand des Standorts beurteilt werden, wie ein Wurzelsystem ausgebildet ist. An städtischen Standorten, dem Untersuchungsschwerpunkt der vorliegenden Arbeit, tritt zudem hinzu, dass auf kleinstem Raum sehr heterogene Standortbedingungen vorliegen können. Dadurch und durch die anthropogene Bodengestaltung vergrößert sich die Vielfalt potentieller Untersuchungsstandorte für die Insitu-Messungen. Dementsprechend werden die Untersuchungsstandorte so gewählt, dass sie Standorte von ungestörten Flächen bis hin zu Extremstandorten umfassen. Es kann aber vor der erfolgten Sichtung der Messergebnisse nicht beurteilt werden, welche Morphologie die erfassten Wurzelsysteme aufweisen (vgl. Kapitel 2.1.1). Die Messungen entsprechend Abbildung 3.1 Schwerpunkt 2 wurden hauptsächlich in einer Göttinger Parkanlage, der Schillerwiese, durchgeführt. Untersuchungsbeispiele zur Verifikation stammen zusätzlich vom Landwehrkanal in Berlin, sowie aus Nimwegen und Fulda. Weitere Messungen im Rahmen von Schwerpunkt 3 sind im Forstbotanischen Garten der Universität Göttingen und Goldschmidtstraße in Göttingen durchgeführt worden. Die Gegebenheiten auf den einzelnen Messflächen können den Tabellen 3.1 und 3.2 entnommen werden, Fotos und Luftbilder mit der genauen Lage der einzelnen Messflächen befinden sich im Anhang (Abbildungen A.2 bis A.11). 
3 Material und Methoden

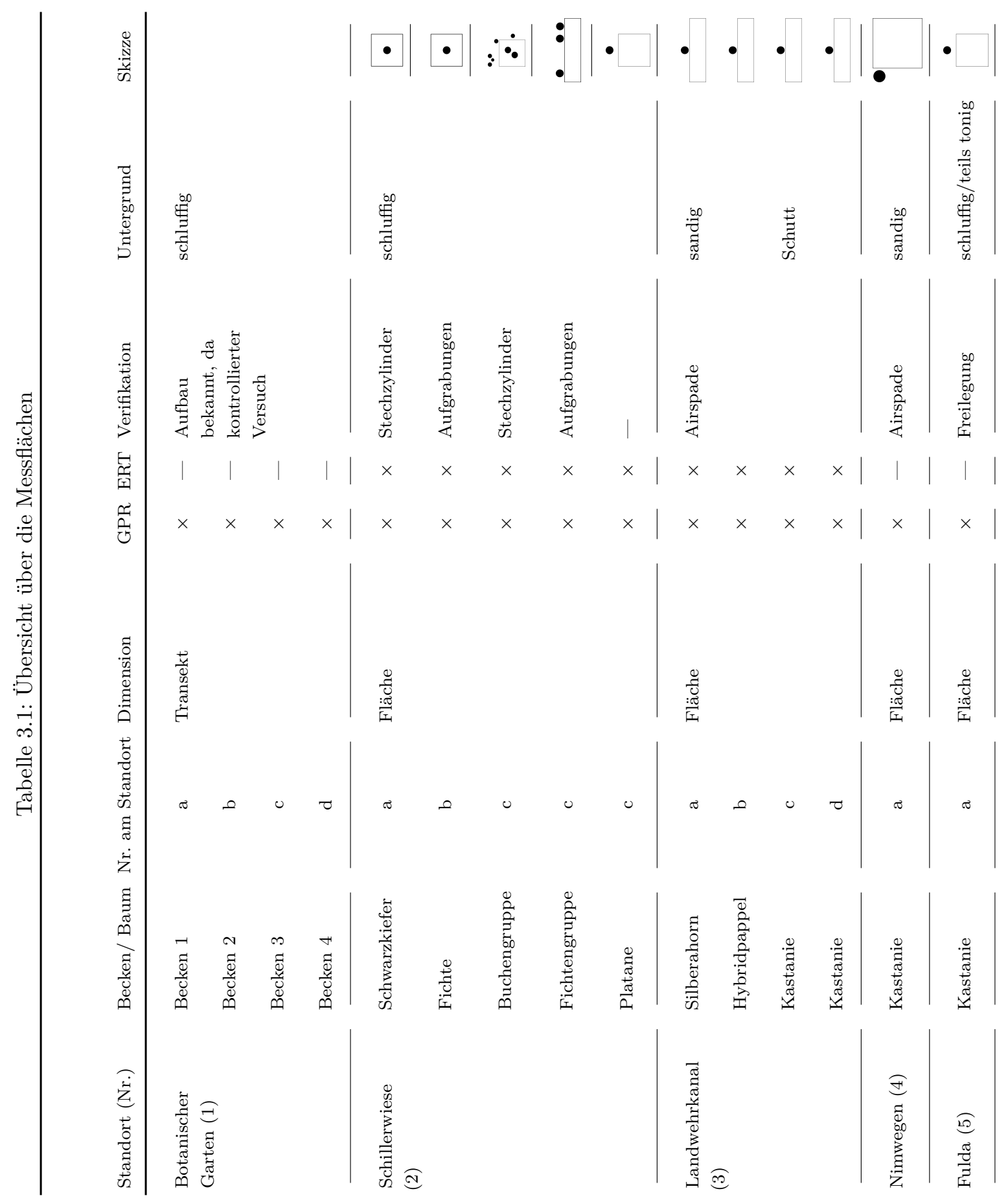




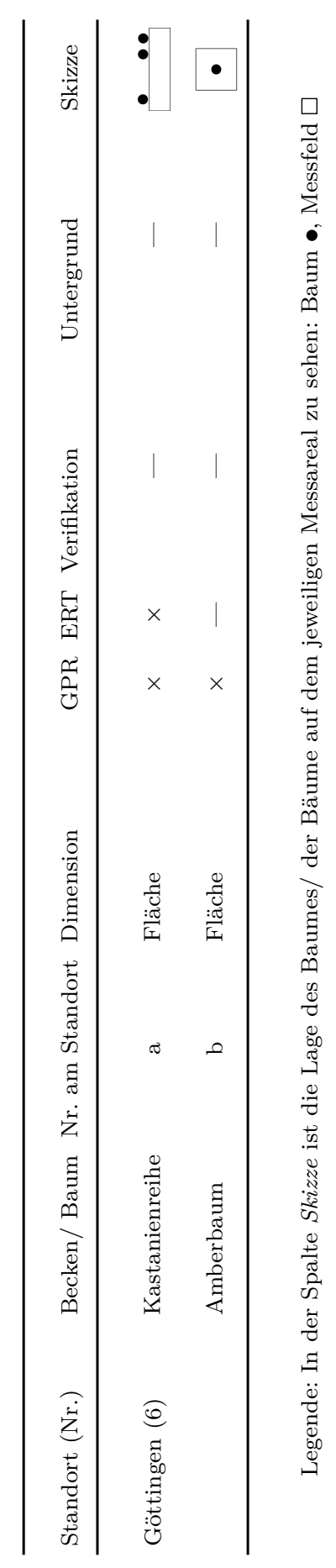


Tabelle 3.2: Maße der Messflächen

\begin{tabular}{|c|c|c|c|c|c|}
\hline Standort & Messfläche & Maße (GPR) & Rasterabstand (GPR) & Maße (ERT) & Elektrodenabstand (ERT) \\
\hline \multirow[t]{5}{*}{ Schillerwiese } & Schwarzkiefer & $12 \mathrm{~m} \times 12 \mathrm{~m}$ & $15 \mathrm{~cm}$ & $12 \mathrm{~m} \times 12 \mathrm{~m}$ & $25 \mathrm{~cm}$ \\
\hline & Fichte & $12 \mathrm{~m} \mathrm{x} 12 \mathrm{~m}$ & & $12 \mathrm{~m} \mathrm{x} 12 \mathrm{~m}$ & \\
\hline & Buchengruppe & $11,5 \mathrm{~m} \times 19,5 \mathrm{~m}$ & & $12 \mathrm{~m} \times 12 \mathrm{~m}$ & \\
\hline & Fichtengruppe & $6 \mathrm{~m} \times 24 \mathrm{~m}$ & & $6 \mathrm{~m} \times 24 \mathrm{~m}$ & \\
\hline & Platane & $6 \mathrm{~m} \times 12 \mathrm{~m}$ & & - & - \\
\hline \multirow[t]{4}{*}{ Landwehrkanal } & Silberahorn & $5,7 \mathrm{~m} \mathrm{x} 27,5 \mathrm{~m}$ & $15 \mathrm{~cm}$ & $5,6 \mathrm{~m} \times 34,3 \mathrm{~m}$ & 0,7 m(längs); 0,25 m(quer) \\
\hline & Hybridpappel & $3,6 \mathrm{~m} \mathrm{x} 18 \mathrm{~m}$ & & $3,8 \mathrm{~m} \times 17,15 \mathrm{~m}$ & $0,35 \mathrm{~m}$ (längs); $0,2 \mathrm{~m}$ (quer) \\
\hline & Große Kastanie & $3 \mathrm{~m} \times 15 \mathrm{~m}$ & & $3 \mathrm{~m} \times 15 \mathrm{~m}$ & $0,25 \mathrm{~m}$ \\
\hline & Kleine Kastanie & $3,6 \mathrm{~m} \times 6 \mathrm{~m}$ & & $3,6 \mathrm{~m} \times 6 \mathrm{~m}$ & $0,25 \mathrm{~m}$ \\
\hline Nimwegen & Kastanie & $2,7 \mathrm{~m} \times 3,6 \mathrm{~m}$ & $15 \mathrm{~cm}$ & - & - \\
\hline Fulda & Fulda & $6 \mathrm{~m} \times 11,4 \mathrm{~m}$ & & - & - \\
\hline \multirow[t]{2}{*}{ Göttingen } & Kastanienreihe & $3,6 \mathrm{~m} \times 39 \mathrm{~m}$ & & - & - \\
\hline & Amberbaum & $12 \mathrm{~m} \mathrm{x} 12 \mathrm{~m}$ & & - & - \\
\hline
\end{tabular}

\subsubsection{Versuchsbecken}

Die Anlage besteht aus mit Beton eingefassten Becken mit Innenkantenlängen von 1,2 $\mathrm{m} \times$ 5,0 m. Es wurden drei Becken mit einer Bodentiefe von 2,2 $\mathrm{m}$ und ein Becken mit einer Tiefe von 1,2 m genutzt. Der Boden der Becken ist mit einer $30 \mathrm{~cm}$ dicken Drainageschicht aus grobem Kies bedeckt, die durch eine von einer feinkörnigen Sandschicht bedeckten Stoffmatte vor Sedimenteintrag geschützt wird (Pollmeier 2009). In den für die Versuche genutzten Becken ist das natürliche Substrat des umgebenden Geländes eingebracht, bei dem aufgrund der künstlichen Aufschüttung keine gewachsene Horizontierung vorliegt. Deshalb kann für die Becken lediglich eine Bodenart und kein Bodentyp ausgewiesen werden. Nach Pollmeier (2009) handelt es sich dabei um mittleren schluffigen Ton (Tu3). Die Möglichkeit, in den Lysimeterbecken unterschiedliche Bodenwassergehalte herzustellen, wurde nicht genutzt. Alle Becken wurden mit einem mobilen Plexiglasdach vor Niederschlagseinfluss geschützt, wodurch die Bedingungen während der Messungen annäherend konstant gehalten werden konnten. Der genaue Aufbau in allen Becken kann Abbildung 3.2 entnommen werden. Detailliertere Informationen sind Tabelle A.3 und den Fotos im Anhang zu entnehmen. Das Probenmaterial besteht aus frisch geschnittenen Ästen unterschiedlicher Baumarten, bei denen der Wasserverlust durch Verwendung des Wundverschlussmittels LacBalsam vermieden wird. Es handelt sich aufgrund der Ähnlichkeit zu 
Becken 13

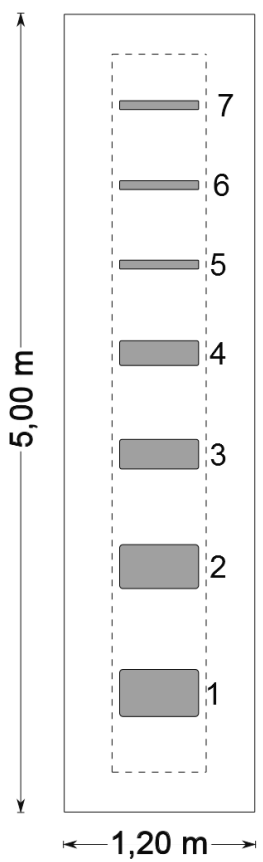

Becken 14

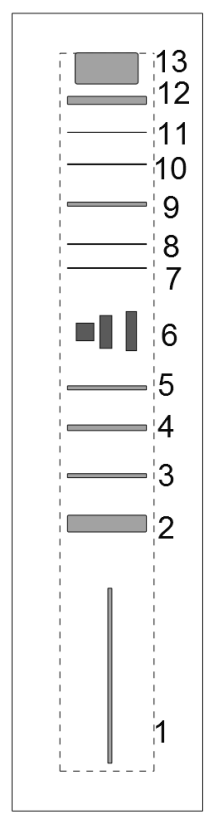

Becken 15

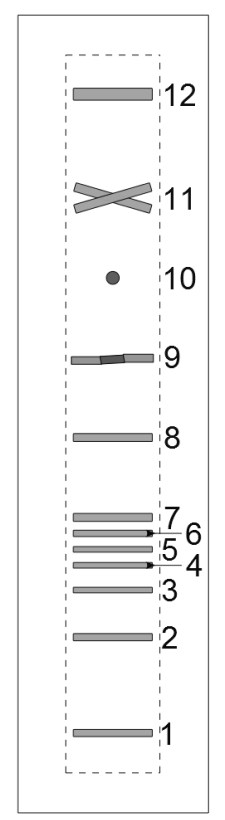

\section{Becken 16}

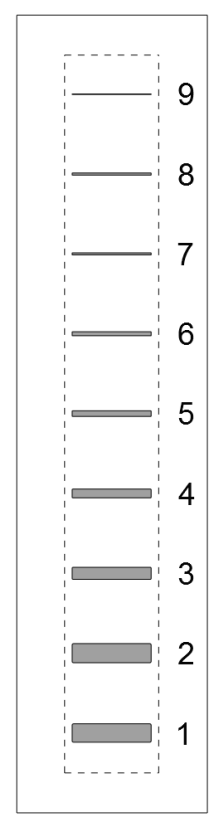

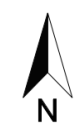

$\square$ Wurzelattrappe Aushubsgrenze Lysimeterbecken

Abbildung 3.2: Anordnung der Wurzelattrappen in den Versuchsbecken- die genauen Positionen und Dimensionen der einzelnen Objekte können Tabelle A.3 entnommen werden.

Wurzelholz um Äste zerstreutporiger Baumarten.

Becken 1 Der Hintergrund der Versuchsanordnung in Becken 1 ist die Ermittlung des Minimaldurchmessers, bis zu dem Wurzeln detektiert werden können. Becken 1 beinhaltet 9 Äste mit abnehmenden Durchmessern (12,5 cm-0,8 cm). Der horizontale Abstand zwischen den einzelnen Wurzelattrappen beträgt konstant $50 \mathrm{~cm}$. Die Unterseiten der Äste liegen in einer konstanten Tiefe von $40 \mathrm{~cm}$.

Becken 2 Der Hintergrund der Versuchsanordnung in Becken 2 ist die Ermittlung des horizontalen Minimalabstands, den zwei Wurzeln zueinander aufweisen müssen, damit sie als eigenständige Objekte im Radargramm abgebildet werden. Des Weiteren wird untersucht, ob sich übereinanderliegende Wurzeln im Radargramm unterscheiden lassen. Für den südlichen Teil von Becken 2 wurden 7 Äste mit Stärken von $4,0 \mathrm{~cm}-5,0 \mathrm{~cm}$ gewählt, deren horizontale Abstände von anfangs $60 \mathrm{~cm}$ sukzessiv bis auf $10 \mathrm{~cm}$ verringert wurden, wobei hier die Unterkanten in einer konstanten Tiefe von $40 \mathrm{~cm}$ liegen. Die Anordnung im nördlich angrenzenden Beckenteil umfasst drei Äste mit Durchmessern von $5,0 \mathrm{~cm}-6,0 \mathrm{~cm}$, deren vertikaler Abstand zueinander jeweils $7,0 \mathrm{~cm}$ beträgt. Am nördlichen Ende von Becken 2 befinden sich zwei weitere Äste mit einem Durchmesser von $4,0 \mathrm{~cm}$ in einem Vertikalabstand von $26 \mathrm{~cm}$.

Becken 3 Die Anordnung in Becken 3 besteht neben Wurzelattrappen aus verschiedenen technogenen Materialien, wie sie in städtischen Böden vorkommen. Dadurch ergibt sich die Möglichkeit, zu untersuchen, wie diese im Vergleich zu Baumwurzeln abgebildet werden (siehe 
Abbildung 3.2).

Becken 4 Für eine weiterführende Untersuchung der horizontalen und vertikalen Auflösung (vgl. Becken 1 und 2) wurde ein weiterer Versuch in Becken 4 angelegt. Im Vergleich zu den bisherigen Anordnungen sind in Becken 4 Wurzelttrappen größerer Stärken $(30,0 \mathrm{~cm}-6,0 \mathrm{~cm})$ angeordnet. Dabei variieren der horizontale Abstand und die Tiefenlage der Objekte.

\subsubsection{Schillerwiese Göttingen}

Auf der Schillerwiese, einer Göttinger Parkanlage, wurden Messungen an einer Schwarzkiefer, einer Fichte, einer Platane, einer Buchengruppe und einer Fichtengruppe durchgeführt (vgl. Abbildung A.2 im Anhang). Außer der Buchengruppe, bei der eine Mulchauflage den Untergrund bedeckt, sind die Oberfächen aller Versuchsflächen auf der Schillerwiese grasbewachsen. Die Messfläche Buchengruppe unterscheidet sich darüber hinaus von den übrigen untersuchten Standorten. Hier stehen im näheren Randbereich außerhalb der Messfläche weitere Bäume, deren Wurzelsysteme mit hoher Wahrscheinlichkeit in die Messfläche reichen. Die übrigen Messflächen liegen entweder im Bereich von Solitärbäumen oder wurden als Baumgruppe mit einem größerem Abstand zu den nächsten Bäumen erfasst, wodurch es unwahrscheinlich ist, die Wurzeln anderer Bäume bei den entsprechenden Messungen zu erfassen.

Von den Messflächen auf der Schillerwiese wurden die Schwarzkiefer und die Buchengruppe von Gronwald (2010) im Rahmen einer Bachelorarbeit bodenkundlich untersucht, wobei die an diesen Standorten ermittelten Bodenkennwerte aufgrund der räumlichen Nähe auch für die benachbarten Messflächen auf der Schillerwiese herangezogen werden. Die Bodenart im Umfeld der Schwarzkiefer wird dabei zwischen Ut4 (toniger Schluff) und Lu (schluffiger Lehm) eingeordnet. Die Lagerungsdichten des Substrat an diesem Standort wurden als sehr gering bis mittel (Ld3) eingestuft. Beim Oberboden $(0 \mathrm{~cm}-2 \mathrm{~cm})$ im Umfeld der Buchengruppe handelt es sich Gronwald (2010) zufolge um lehmigen Sand (S13). In tieferen Lagen wird dieser von lehmig-schluffigen Sand (Slu) bzw. tonigen Schluff (Ut3) abgelöst. Die Lagerungsdichte wird als mittel (Ld3) eingestuft. Gronwald (2010) nimmt an, dass der höhere Sandanteil im Vergleich zum Umfeld der Schwarzkiefer durch anthropogenen Eintrag aus dem Bereich des Spielplatzes verursacht worden ist.

\subsubsection{Landwehrkanal Berlin}

Der Landwehrkanal ist ein Berlin von Ost nach West durchlaufender Seitenkanal der Spree, der auf einer Strecke von 10,73 km die Spree am Osthafen mit der Spree am Spreekreuz Charlottenburg verbindet. Entlang dieser Wasserstraße wurden mit dem Einsteinufer und dem Corneliusufer zwei Uferabschnitte mit unterschiedlicher Topographie, verschiedenem Untergrundaufbau und Baumbestand untersucht (Abbildung A.5 und A.6). Am Einsteinufer wurden zwei Solitärbäume, ein Silberahorn und eine Hybridpappel, am Corneliusufer die Standorte von zwei Rosskastanien untersucht.

Für die Darstellung des Untergrundaufbaus auf den Messflächen am Einsteinufer wird auf die 
bodenkundlichen Untersuchungen von Barsig et al. (2008) zurückgegriffen. Die Bodenverhältnisse auf den Messflächen am Corneliusufer können anhand von geotechnischen Schnittbildern (Abbildung A.5 und A.6) aus einem Baugrundgutachten der Bundesanstalt für Wasserbau (BAW) vom 24.06.2008 (Liebetruth und Eißfeldt 2008) dargestellt werden, welche ergänzend auch für die Messflächen am Einsteinufer herangezogen werden.

Einsteinufer Der untersuchte Abschnitt des Einsteinufers mit dem Silberahorn und der Hybridpappel befindet sich zwischen Marchbrücke und Charlottenburger Tor auf der südlichen Kanalseite in Höhe des Gebäudes der Akustikprüfstelle der TU Berlin. Barsig et al. (2008) haben das Umfeld einer Pappel im selben Uferabschnitt des Einsteinufers untersucht, sowie drei Baumumfelder in einem weiteren, nahegelegenen Uferabschnitt. Alle Böden entlang des Uferabschnitts werden von den Autoren als anthropogen aufgetragene, mittelsandige Böden mit Umlagerungsspuren ausgewiesen, woraus sich für das Einsteinufer eine gewisse Homogenität des Untergrundaufbaus ableiten lässt. Da die in der vorliegenden Arbeit untersuchten Baumstandorte zwischen den von Barsig et al. (2008) untersuchten Probepunkten liegen, erscheint es wahrscheinlich, dass die Bodenverhältnisse auch in diesen Bereichen ähnlich sind. Daher wird für die Einstufung der eigenen Messungen auf die Daten von Barsig et al. (2008) zurückgegriffen. Im Folgenden werden die Ergebnisse der bodenkundlichen Untersuchung von von Barsig et al. (2008) an dem Probepunkt wiedergegeben, der den eigenen Untersuchungsflächen am nächsten liegt:

Der Oberboden $(0 \mathrm{~cm}-30 \mathrm{~cm})$ setzt sich aus einem sehr humos, kalkfreien, anthropogen umgelagerten Substrat zusammen, das stellenweise mit kleinen Ziegelresten durchsetzt ist; die Bodenart ist feiner Mittelsand. Unterhalb $(30 \mathrm{~cm}-70 \mathrm{~cm})$ schließt sich ebenfalls feiner Mittelsand als Bodenart an, der Humusanteil geht zurück. Grundwassereinfluss und hydromorphe Merkmale treten hinzu. Der unterliegende Horizont $(70 \mathrm{~cm}-100 \mathrm{~cm})$ wird ebenfalls als feiner Mittelsand ausgewiesen, der im Jahresverlauf fast durchgehend reduzierende Verhältnisse aufweist. In $75 \mathrm{~cm}$ Tiefe liegt hier eine Ziegelsteinschicht vor; der Kapillarsaum befindet sich in einer Tiefe von ca. $80 \mathrm{~cm}$. Dieser Aufbau wird von (Liebetruth und Eißfeldt 2008) bestätigt. So sind im entsprechenden geotechnischen Längsschnitt (Abbildung A.5 die oberen $80 \mathrm{~cm}$ als anthropogener Bodenhorizont (A) mit sandigen, schluffigen und humosen Komponenten ausgewiesen. Der geotechnische Längsschnitt verdeutlicht zudem die niedrige Vertikaldistanz zwischen Bodenoberfläche und Kanalpegel in diesem Uferabschnitt.

Corneliusufer Das Corneliusufer befindet sich etwa 2,7 km vom westlichen Ende des Landwehrkanals (Kreuzungsbereich) entfernt. Am Nordufer dieses Standorts wurden die Baumumfelder von zwei unterschiedlich dimensionierten Kastanien untersucht. Wie im geotechnischen Längsschnitt ersichtlich wird (Abbildung A.6), weicht dieser Uferbereich in seiner Beschaffenheit deutlich vom Einsteinufer ab. Zum einen besteht hier eine größere Vertikaldistanz zwischen Bodenoberfläche und Kanalpegel. Zum anderen liegt hier mit einer etwa 3,0 m-4,0 m mächtigen anthropogenen Auffüllungsschicht geringer bis mittlerer Lagerungsdichte ein anderes Substrat vor. 


\subsubsection{Weitere Messflächen}

Kastanie Nimwegen Nach Sturmschäden an einer Kastanienallee in der Archipelstraat in Nimwegen wurden vom Baumgutachter Jan Willem de Groot Untersuchungen an verschiedenen Bäumen durchgeführt. In diesem Rahmen konnte eine Bodenradaruntersuchung an einem der Bäume durchgeführt werden. Es wurde untersucht, ob sich die Grobwurzelverläufe in dem gepflasterten Bereich mittels Georadar rekonstruieren lassen (vgl. Abbildung A.8.

Kastanie Fulda Ein weiterer Baum, in dessen Umfeld Messungen durchgeführt worden sind, ist eine Rosskastanie in Fulda. Die Untersuchung an diesem Baum fand im Zusammenhang mit einer geplanten Bautätigkeit im Umfeld der Kreuzkirche Fulda statt. Dabei sollten die Wurzelverläufe der Kastanie im Vorfeld der Bautätigkeit geortet werden, um die Anlage der für den Bau notwendigen Punktfundamente baumschonend zu gestalten (vgl. Abbildung A.9).

Kastanienreihe Göttingen In der Goldschmidtstraße in Göttingen wurde eine straßenbegleitende Kastanienreihe vor dem Gebäude der Physikalischen Fakultät untersucht. Hintergrund der Messung war es, Vergleichsdaten zu den anderen untersuchten Kastanienstandorten zu erhalten. Der Untergrund des Untersuchungsstandorts ist grasbewachsen (vgl. Abbildung A.10).

Amberbaum Forstbotanischer Garten Göttingen Der untersuchte Amberbaum ist Teil des Forstbotanischen Gartens der Universität Göttingen (vgl. Abbildung A.11). Er befindet sich nicht im Kernstück der Anlage, sondern steht als Teil der Sammlung auf dem Nordcampus der Universität Göttingen auf der Grünfläche vor dem Gebäude Büsgeninstitut der Forstwissenschaftlichen Fakultät. Der Untergrund der rasenbewachsenen Fläche wurde nach Auskunft des technischen Leiters des Forstbotanischen Gartens, Herrn Meng, bei der Anlage des Nordcampus künstlich aufgeschüttet und stark verdichtet. Die Bodenart im unmittelbaren Umfeld des Baumes wurde von Pollmeier (2009) bis in eine Tiefe von $40 \mathrm{~cm}$ als schluffiger Lehm $(\mathrm{Lu})$ und zwischen $40 \mathrm{~cm}-$ $50 \mathrm{~cm}$ als schwach schluffiger Sand mit Skelettanteil bestimmt. Ab einer Tiefe von $50 \mathrm{~cm}-55 \mathrm{~cm}$ steht Kalkstein an.

\subsection{Georadarmessungen}

\subsubsection{Messsystem}

Für die GPR-Aufnahmen wurde das Radarsystem SIR-3000 von GSSI mit einer bodengekoppelten $400 \mathrm{MHz}$-Antenne verwendet (vgl. Abbildung 3.3). Bei der Antenne handelt es sich um eine bistatische Antenne. Da Sende- und Empfangsantenne mit festem Abstand in einem Antennenkasten montiert sind, können ohne zweites Exemplar lediglich Messungen im Common-Offpoint-Modus realisiert werden. Die Antenne und Steuereinheit des verwendeten Bodenradarsystems sind an einem Messwagen befestigt und über ein optisches Glasfaserkabel miteinander verbunden (Abbildung 3.3). Die Wegmessung erfolgt über ein Impulse gebendes Messrad an einem der Hinterräder. Für die Bearbeitung, Aufbereitung und Auswertung der gewonnenen Daten wurde das Programmpaket RADAN 6.6 von GSSI (GSSI 2007) eingesetzt. 


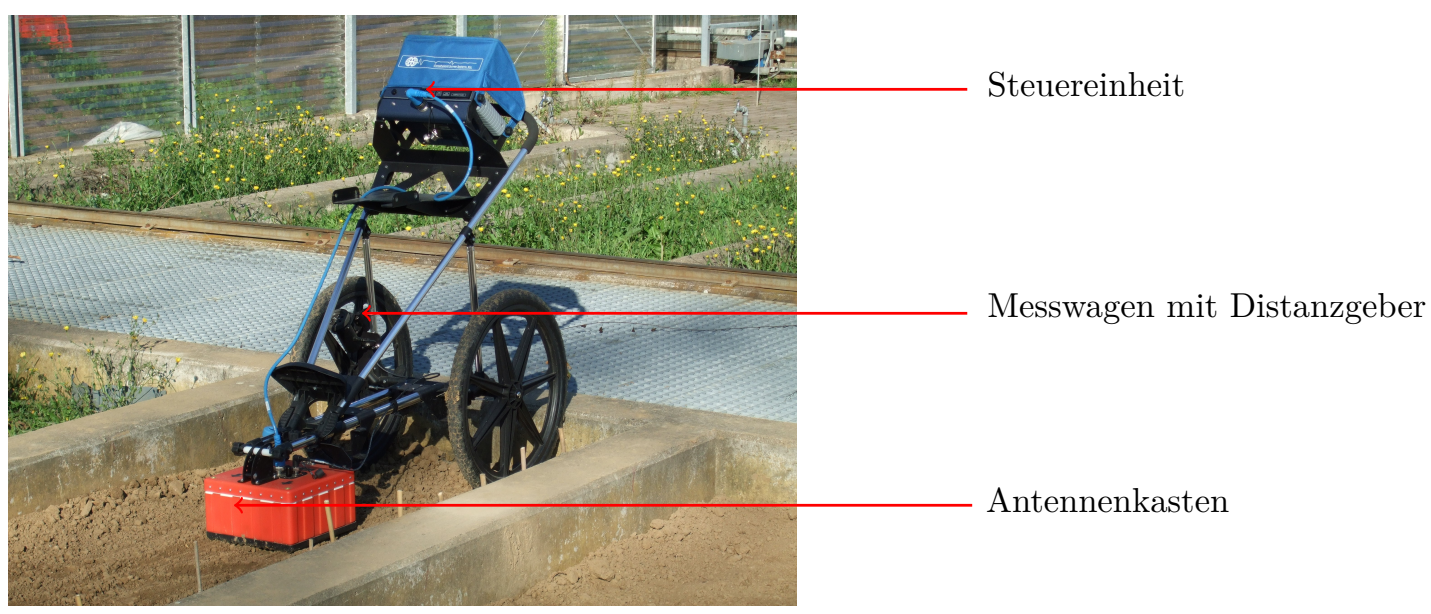

Abbildung 3.3: Eingesetztes Georadarsystem GSSI SIR-3000 mit Messwagen und $400 \mathrm{MHz}-$ Antenne.

\subsubsection{Durchführung der Messungen}

\subsubsection{Allgemein}

Vor Beginn der eigentlichen Messung wurde eine Testbefahrung der zu erfassenden Fläche durchgeführt, um die Signalverstärkung (gain), so einstellen zu können, dass die Amplituden vollständig aufgezeichnet werden und kein Übersteuern ('clipping) stattfindet. Weiterhin wurden die gain-Einstellungen so gewählt, dass bei Testmessungen direkt vor der eigentlichen Datenaufzeichnung deutliche Hyperbeln aufgezeichnet werden konnten. Das Laufzeitfenster wurde bei den einzelnen Messungen so an die vorherrschenden Bedingungen angepasst, dass am Ende des erfassten Zeitfensters noch ein ausreichendes Signal:Noise-Verhältnis vorliegt. Für die Anzahl von Scans pro Streckenintervall wurden 200 Scans/m gewählt, da mit diesem Wert bei Testmessungen aussagekräftige Ergebnisse erzielt werden konnten.

\subsubsection{2D-Georadarmessungen in den Versuchsbecken}

Aufgrund der Abmessungen des mit der Antenne verbundenen Messwagens (vgl. Abbildung 3.3 war es nicht möglich, das gesamte Becken mit einer Messung in Längsrichtung zu erfassen. Stattdessen erfolgten pro Becken zwei Befahrungen, ausgehend vom nördlichen und südlichen Rand (vgl. Abbildung 3.4) um sicherzustellen, dass alle eingebrachten Versuchsobjekte erfasst werden. Die aufgenommenen Transekte fallen dementsprechend jeweils etwa 1,16 m (Abstand von der Antennenaußenkante zum Kontaktpunkt des Hinterrads an der Betoneinfassung plus $6 \mathrm{~cm}$ - Plastikrohr bzw. Siebeinsatz vor den Einfassungen der Lysimeterbecken) kürzer aus als die Innenlänge der Becken.

Die orthogonale Lage der Messrichtung zu den Zielobjekten führt dabei zu den stärksten Amplituden im empfangenen Signal und dazu, dass beim Überfahren von Objekten die charakteristischen Diffraktionshyperbeln entstehen (vgl. Kapitel 2.3.2). 


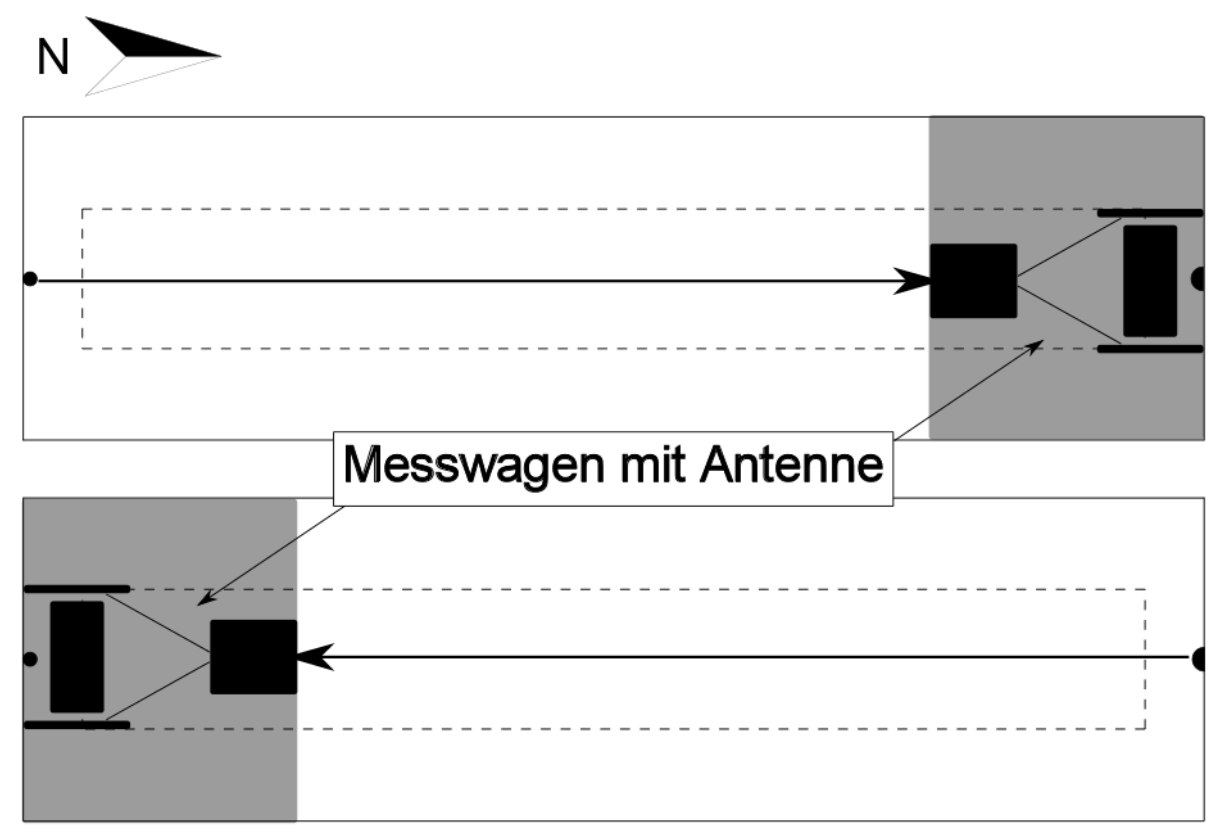

Abbildung 3.4: Schematische Darstellung der Georadarmessungen in den Versuchsbecken. Die aufgrund der Abmessungen des Wagens nicht erfassbare Fläche ist jeweils grau unterlegt (Aufsicht).

\subsubsection{3D-Messungen zu grundlegenden Fragestellungen und 3D-Messungen auf ausgewählten Standorten}

Die weiteren Untersuchungsstandorte sind dreidimensional in Form von Rastern erfasst worden. Die Ermittlung eines optimalen Aufnahme- und Auswertungsschemas ist Teil der vorliegenden Arbeit und wird im Ergebnisteil in den Kapiteln 4.1 und 4.2 erläutert.

Die Raster setzen sich aus mehreren parallel und orthogonal zueinander aufgenommenen Transekten zusammen. Die Aufzeichnung der einzelnen Transekte eines Rasters erfolgte wie die zweidimensionalen Messungen in den Versuchsbecken. Die Messflächen konnten dabei in den meisten Fällen nicht mit einem einzelnen Raster abgedeckt werden, sondern mussten in mehrere Einzelraster (vgl. Abbildung 3.5a) unterteilt werden, um Messhindernisse zu umgehen. Diese waren auf jeder Messfläche mindestens in Gestalt des zu untersuchenden Baumes vorhanden. Teilweise wurde aber auch ein großes Messraster gewählt, bei dem die Transekte auf Höhe des Baumes abgebrochen worden sind. Nach Aufnahme des Rasters wurden die fehlenden Teile in Form von einzelnen ergänzenden Transekten erfasst (vgl. Abbildung 3.5b). Wenn Messungen aufgrund der Oberflächenbeschaffenheit nicht durchgeführt werden konnten, wurden stellenweise auch kleinere Flächen ausgespart. Da es bei den Untersuchungen darum geht, unbekannte Strukturen zu visualisieren, war es nicht möglich, die Ausdehnung der jeweiligen Messflächen im Vorfeld so festzulegen, dass die Wurzelsysteme in jedem Fall komplett abgebildet werden. Stattdessen ist davon auszugehen, dass bei einigen Messbeispielen die Wurzelsysteme deutlich über die erfassten Flächen hinausragen. Als Orientierung bei der Festlegung der Messflächen diente in der Regel die Ausdehnung der Baumkrone.

Die genauen Details zur Durchführung der einzelnen Messungen zu den Schwerpunkten 2 und 3 in Abbildung 3.1 werden für eine bessere Lesbarkeit direkt vor den entsprechenden 


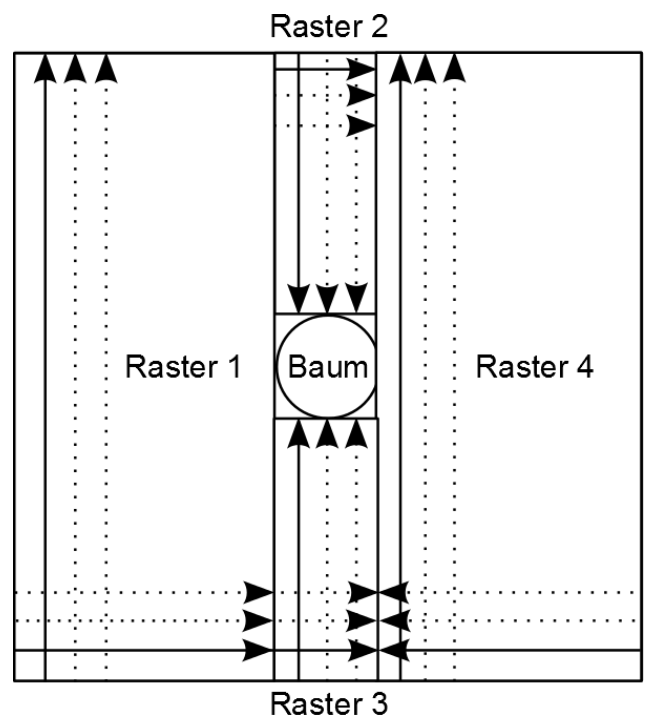

a) Aufnahme mit mehreren Einzelrastern

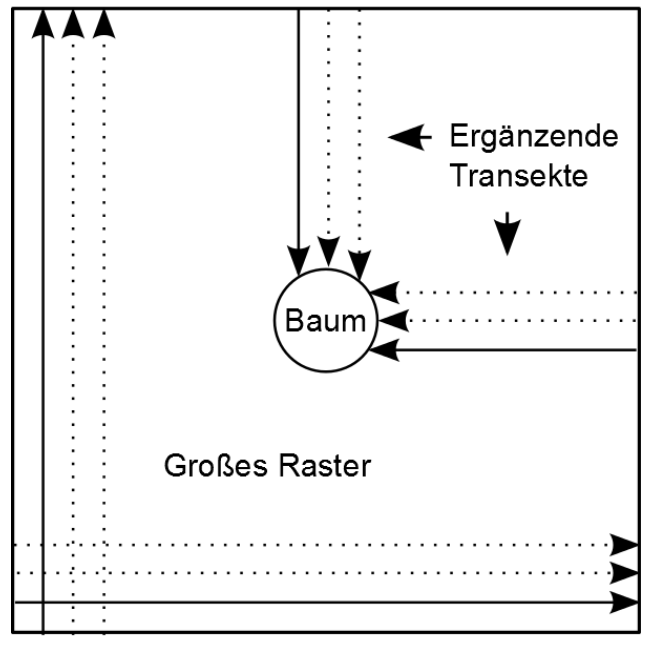

b) Aufnahme mit einem großen Rastern und ergänzenden Transekten auf Höhe des Baumes

Abbildung 3.5: Verschiedene Möglichkeiten zur Durchführung einer dreidimensionalen Bodenradarmessung im Umfeld eines Baumes.

Ergebnisdarstellungen erläutert.

\subsubsection{Datenbearbeitung, Ergebnisdarstellung und Auswertung}

Sofern die präsentierten Ergebnisse nicht als unbearbeitete Rohdaten gekennzeichnet worden sind, liegen der Bearbeitung und Visualisierung der Georadardaten die in Kapitel 2.3.2.3 und 2.3.2.4 beschriebenen Verfahren zugrunde.

2D-Georadarmessungen in den Versuchsbecken Für die Darstellungen in Kapitel 4.1.1 wurden die beiden Einzelmessungen (vgl. Abbildung 3.4 für jedes Becken mit dem Bildbearbeitungsprogramm GIMP zu einem Gesamtradargramm zusammengefügt. Die resultierende Längsausdehnung der zusammengefügten Transekte liegt zwischen 4,50 m und 4,56 m. Die Auswertung der Messergebnisse erfolgt, indem die Radargramme (B-Scans) und ausgewählte wavelets (A-Scans) rein visuell auf ihren Informationsgehalt in Bezug auf die bekannten Positionen der Versuchsobjekte überprüft werden. Aus didaktischen Gründen werden dabei zuerst die B-Scans und daran anschließend die A-Scans dargestellt. Dadurch kann die Aufnahmeposition der A-Scans besser in den B-Scans verortet werden.

Für die Korrelation von Zwei-Wege-Laufzeiten und Bodentiefen wurden zwei verschiedene Berechnungsverfahren eingesetzt. Bei Reflexionswegen, die direkt zwischen Sendeantenne, dem Objekt im Boden und der Empfangsantenne verliefen, wurde die doppelte Tiefe der Objektoberkante für $s$ gewählt, da sich Gleichung 2.2 in $v=\frac{2 s}{t}$ umstellen lässt. In den Fällen, in denen angenommen wurde, dass eine umlaufende Welle (vgl. Abbildung 2.7) vorliegt, wurde für $s$ zur doppelten Distanz bis zur Objektoberkante zusätzlich der Weg um die jeweilige Wurzel (Wurzelumfang) addiert. Der Wurzelumfang wurde dabei mit $u=d \pi$ aus dem Durchmesser $d$ 
berechnet.

3D-Messungen zu grundlegenden Fragestellungen und 3D-Messungen auf ausgewählten

Standorten Für die Analyse der dreidimensionalen Georadarmessungen werden überwiegend nach dem in Abbildung 3.6 dargestellten Schema erstellte Zeitscheiben (time-slices) verwendet.

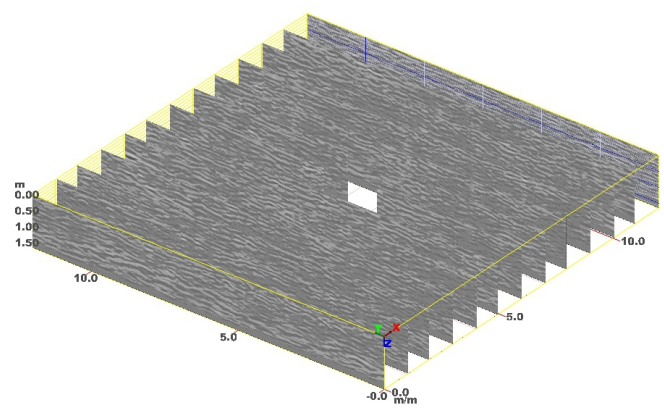

a) x-Richtung

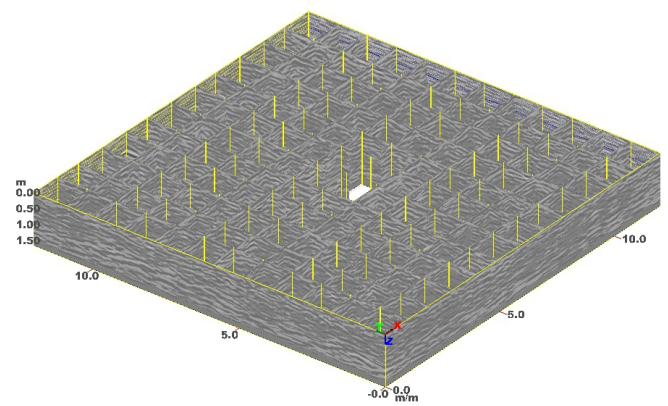

c) Verschnitt von $x$ - und y-Richtung

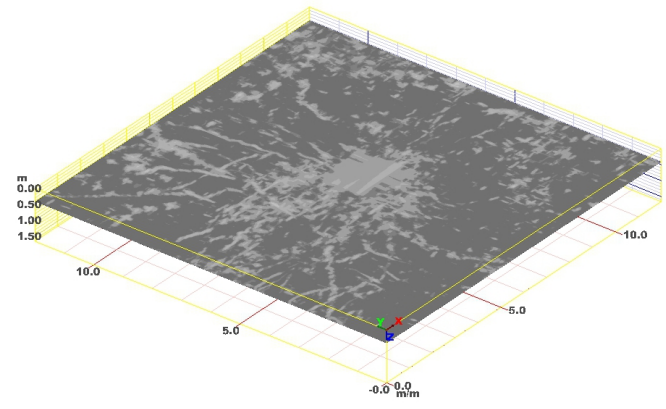

e) Freistellung time-slice

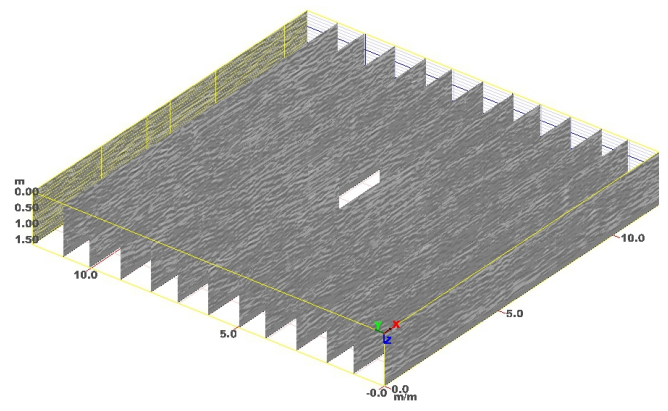

b) y-Richtung

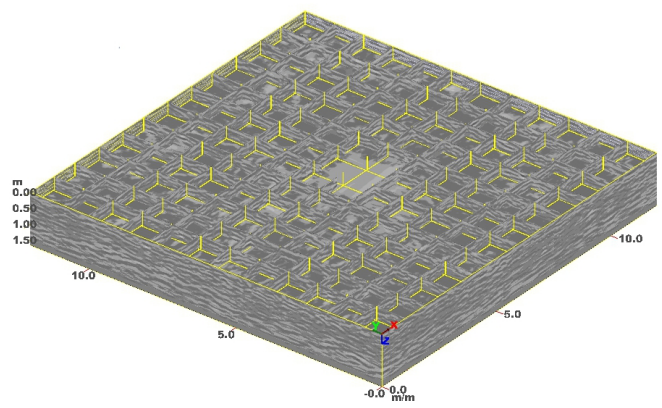

d) Auswahl Horizontalschnitt

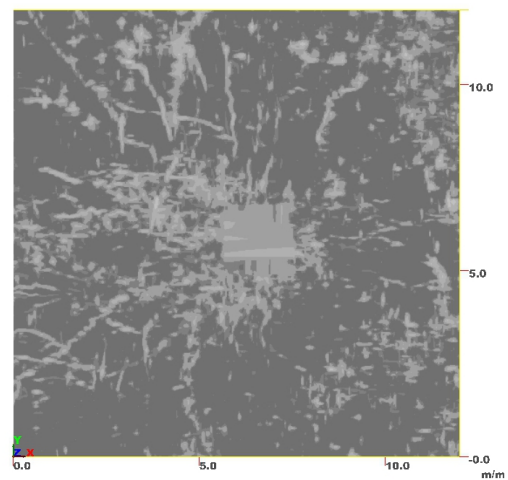

f) Drehen und Draufsicht

Abbildung 3.6: Erstellung einer Zeitscheibe als Ergebnisdarstellung von Bodenradarmessungen zur Wurzelortung.

Die Herausforderung bei einer solchen Darstellung liegt darin, dass sich hier zusammenhängende Objekte innerhalb eines bestimmten Zeitabschnitts identifizieren lassen. Aufgrund des Zusammenhangs $s=\frac{v t}{2}$ (vgl. Kapitel 2.3.2 entspricht dieser auch einer bestimmten Tiefenstufe. Baumwurzeln müssen aber nicht unbedingt innerhalb einer solchen Zeitscheibe verlaufen. Statt- 
dessen können diese, wie es beispielsweise bei dem Idealtypus eines Herzwurzelsystems der Fall ist, auch ein deutliches Tiefenwachstum aufweisen (vgl. Kapitel 2.1.1).

Abbildung 3.7 zeigt einen solchen Fall exemplarisch. Dabei wird deutlich, dass insbesondere zur

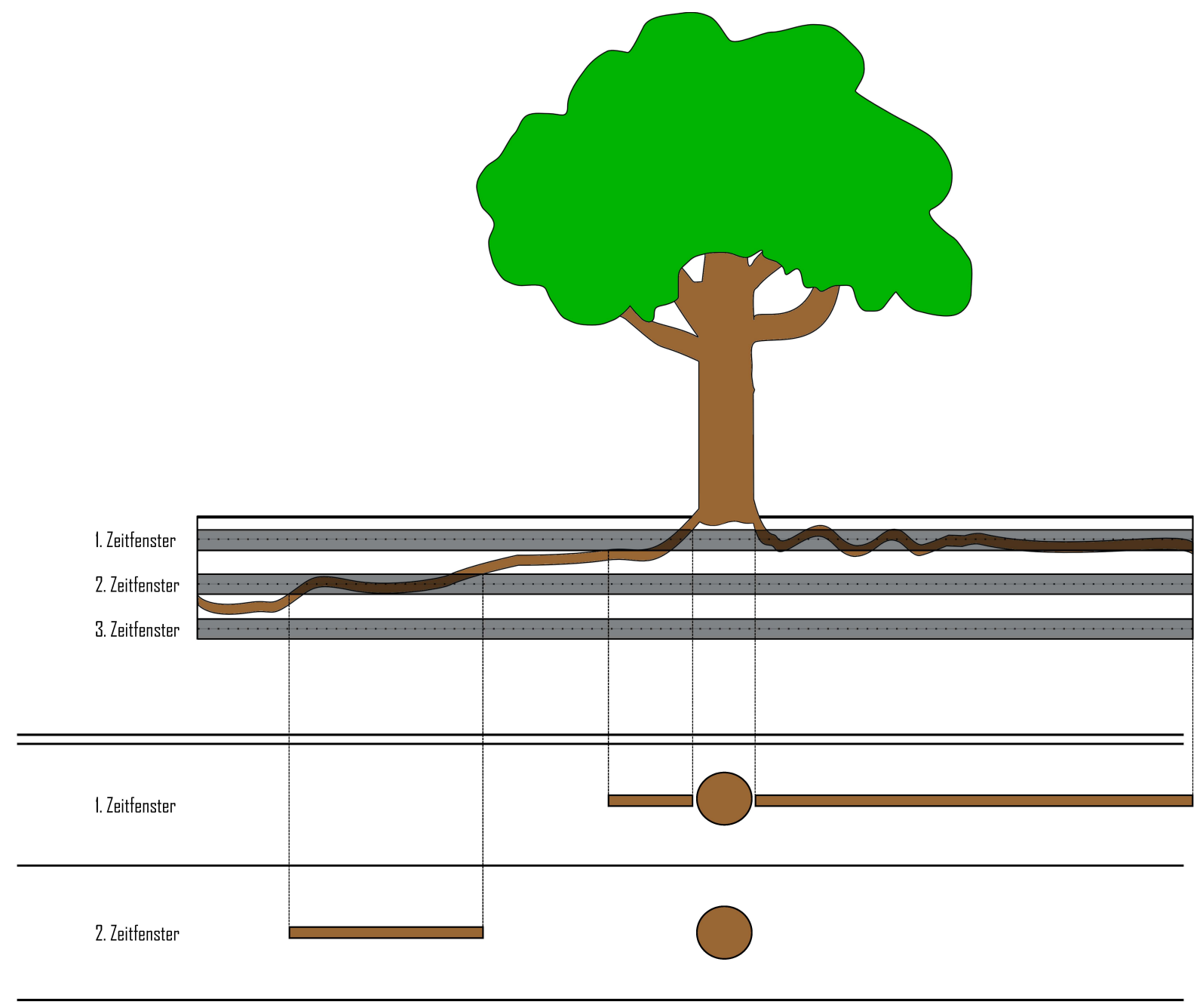

3. Zeitfenster

Abbildung 3.7: Projektion von verschiedenen Laufzeitfenstern in die xy- Ebene (Zeitscheibendarstellung) bei Georadarmessungen zur Wurzelortung. Nur beim 1. Zeitfenster gelingt eine geeignete Visualisierung.

Visualisierung von girlandenförmig in verschiedenen Tiefenstufen verlaufenden Wurzeln und steil abtauchenden Wurzeln sehr große Zeitscheiben gewählt werden müssen, um eine geschlossene Darstellung zu erreichen, bei der die Wurzeln vollständig in einer Zeitscheibe dargestellt werden können. In Abbildung 3.7 gelingt dies nur beim ersten Zeitfenster. Beim zweiten und dritten Zeitfenster kann in der Projektion in eine Zeitscheibe (unterer Bildteil) nur noch ein Teil einer Wurzel oder gar keine Wurzel mehr erkannt werden. Im Fall des zweiten Zeitfensters ist es auch unklar, ob aufgrund der fehlenden Verbindung der Wurzel zum Baum bei einem entsprechenden Messergebnis eine eindeutige Identifikation einer Wurzel gelingen würde.

Eine alternative Darstellungsform wäre die Visualisierung des gesamten erfassten Zeitfensters 
in einer Zeitscheibe. Diese Darstellungsform geht aber mit einem Informationsverlust einher, da nicht mehr bekannt ist, in welchen relativen Tiefenstufen Wurzeln und andere Objekte zueinander liegen. Als Vorgriff auf den Ergebnisteil verdeutlicht dies Abbildung 3.8 am Beispiel der Messfläche Schwarzkiefer (Tabelle 3.1 Baum 2a) anhand eines Vergleichs zwischen der Projektion eines Teilausschnitts (Abbildung 3.8a) und des gesamten aufgenommenen Zeitfensters in einen Horizontalschnitt (Abbildung 3.8b). Für den kleineren Ausschnitt wurden die Parameter

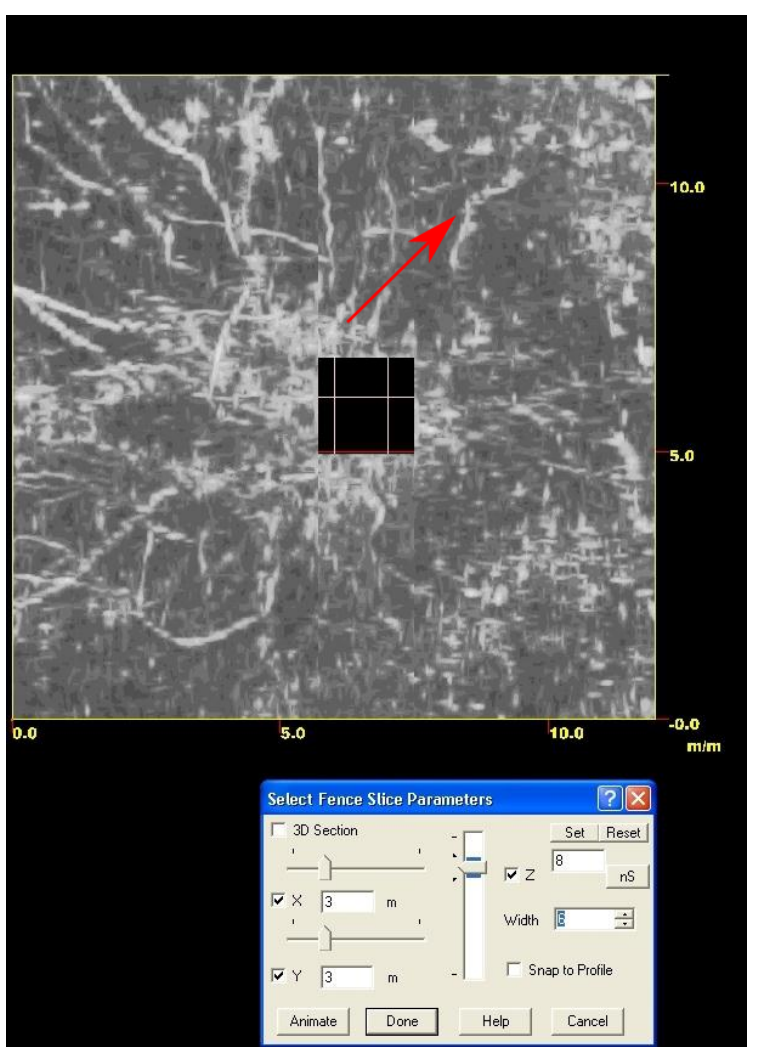

a) Teilausschnitt Daten

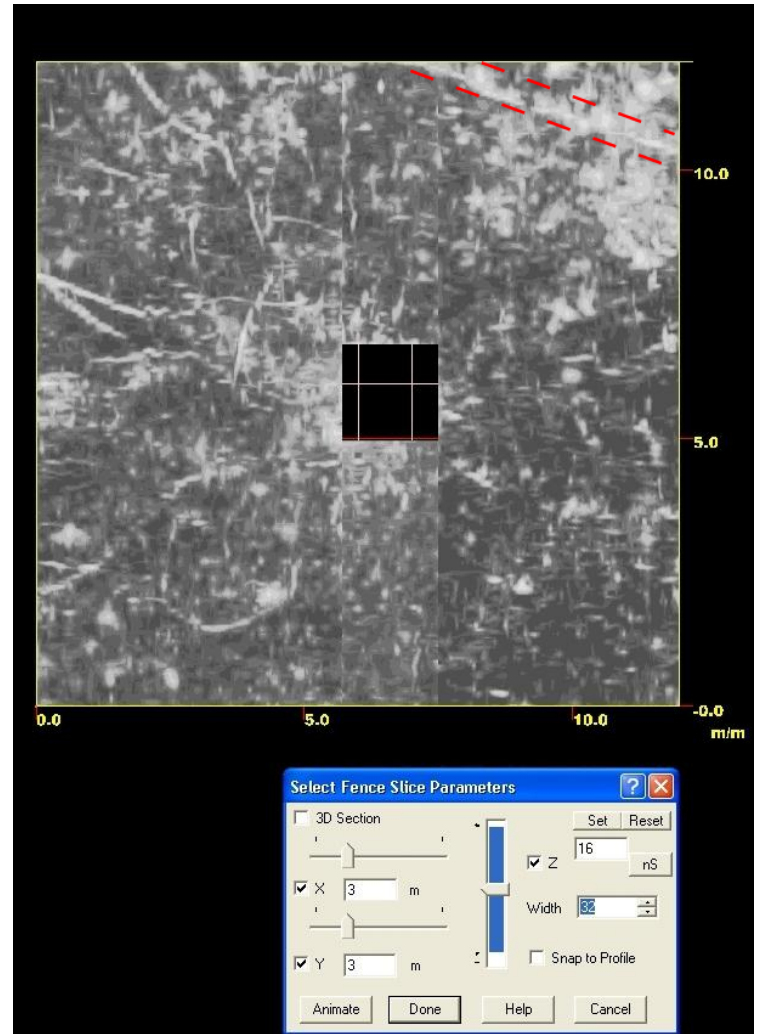

b) Komplettdarstellung

Abbildung 3.8: Zwei Ausschnitte aus dem für die Messfläche Schwarzkiefer erstellten Blockbild mit unterschiedlichen Werten für $\mathrm{z}$ und width. Der linke Horizontalschnitt zeigt einen zur Visualisierung des Wurzelsystems optimierten Ausschnitt, während auf der rechten Seite das erfasste Zeitfenster komplett dargestellt wird. Die Tiefenumrechnung ergibt mit zWert=8; width $=6 ; v=0,07 \mathrm{~m} \mathrm{~ns}^{-1} ; \varepsilon_{r}=18,26$ einen Tiefenbereich von $15,0 \mathrm{~cm}-25,0 \mathrm{~cm}$ für den Teilausschnitt und mit z-Wert $=16$; width $=32 ; v=0,095 \mathrm{~m} \mathrm{~ns}^{-1} ; \varepsilon_{r}=9,97$ ergeben einen Tiefenbereich von $0,0 \mathrm{~cm}-152,0 \mathrm{~cm}$ für den gesamten Laufzeitbereich. In den unteren Fenstern ist jeweils die Auswahl der Parameter 'z' und 'width' für die Erstellung der Zeitscheiben mit RADAN 6.6 zu sehen.

'z' und 'width' so gewählt, dass das Wurzelsystem der Schwarzkiefer möglichst deutlich hervortritt. Bei diesem Vergleich fällt neben der angesprochenen fehlenden relativen Tiefenzuordnung (z.B. ist nicht ersichtlich, ob die Rohrleitung in der oberen rechten Ecke (Abbildung $3.8 \mathrm{~b}$ zwischen ..--) oberhalb oder unterhalb der Wurzeln in der rechten Bildhälfte liegt) ein weiteres Problem auf. So werden Wurzeln, die im linken Horizontalschnitt zu erkennen sind (z.B. Abbildung 3.8a $\longrightarrow$ ), bei der Gesamtdarstellung von anderen flächigen Reflexionen überlagert. Auffällig ist weiterhin, dass an manchen Stellen, an denen in der partiellen Betrachtung Wurzeln gefunden 
werden können, keine Wurzeln in der Komplettvisualisierung vorliegen. In diesen Bereichen gibt es aber auch keine offensichtlich von anderen Objekten hervorgerufenen Reflexionen.

Diese Limitierungen bei der Visualisierung in Form von Zeitscheiben legen den Schluss nahe, bei der Analyse einer Georadarmessung hinsichtlich ihres Informationsgehalts zur Wurzelortung nicht nur eine Zeitscheibe sondern Ausschnitte verschiedener Tiefenstufen zu analysieren.

Um dies bei der Ergebnisdarstellung in der vorliegenden Arbeit zu erreichen, wird für jede Untersuchungsfläche eine Matrix erstellt, in deren Zeilen zu verschiedenen Laufzeiten gehörende Zeitscheiben (xy-Ebenen oder auch Horizontalschnitte) angeordnet sind. Die darin jeweils enthaltenen time-slices werden mit der Auswertesoftware RADAN 6.6 entsprechend Abbildung 3.6 erstellt. Dabei werden ein Basiswert für die Laufzeit (z-Wert in ns) und ein Zeitabschnitt (width in ns) um diesen herum gewählt. In die jeweiligen time-slices werden dann die über einem bestimmten Schwellenwert liegenden Amplituden innerhalb dieses Zeitabschnitts (width/2 oberhalb und unterhalb des z-Werts) projiziert. Für jeden Basiswert werden dann verschiedene width-Werte gewählt, um bei der Gesamtdarstellung fließende Übergänge zu erzielen. Damit wird die Gefahr minimiert, dass Wurzeln, die in verschiedenen Ebenen verlaufen, als Fragmente in einzelnen Zeitscheiben dargestellt werden. Stattdessen steigt die Wahrscheinlichkeit, die Wurzeln als zusammenhängende Objekte zu erfassen. Da bei einer solchen Darstellung von vielen entsprechend kleinen Horizontalschnitten die Details schlechter zur Geltung kommen, wird anhand des schon erwähnten Beispiels Schwarzkiefer, die Analyse einer kompletten Ergebnismatrix vorgestellt (Abbildung 3.9). Bei den übrigen Messflächen werden dagegen prägnante Ausschnitte gewählt und die vollständigen Ergebnismatrizen im Anhang (Kapitel A.4 dargestellt. 1

\footnotetext{
${ }^{1}$ In der digitalen Version der Arbeit können die Abbildungen im Anhang soweit vergrößert werden, dass auch hier Details zur Geltung kommen.
} 


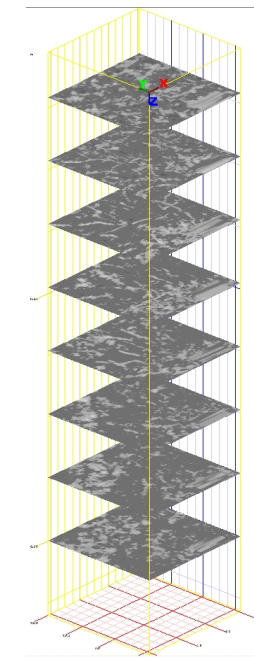

a) Übereinanderliegende Zeitscheiben

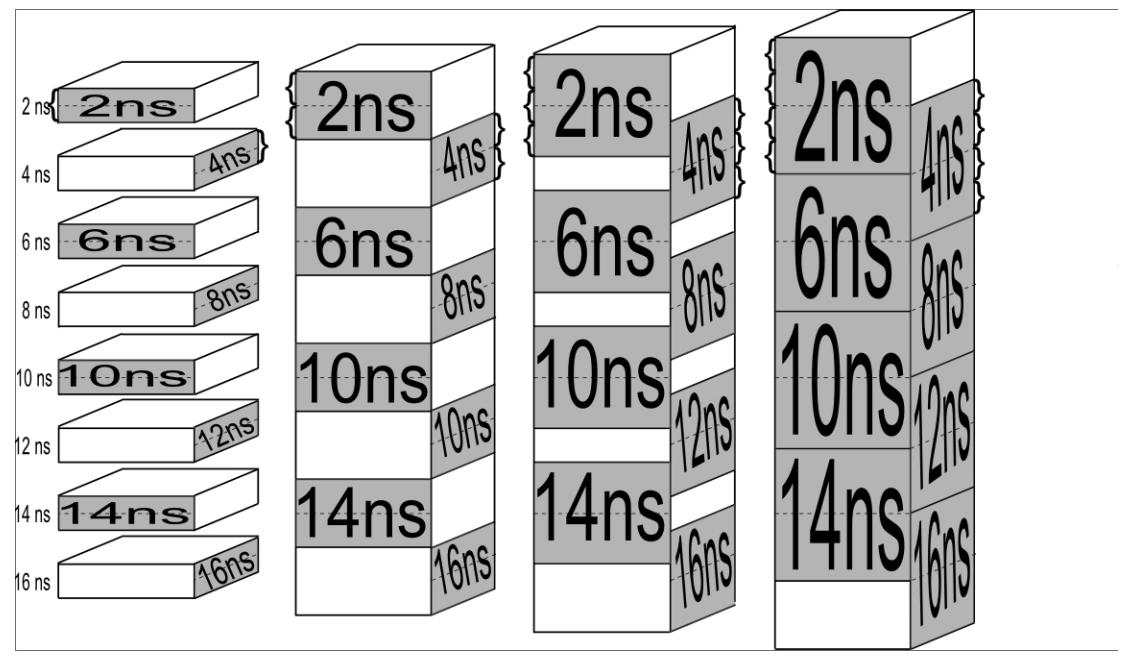

b) Schematische Darstellung der für jede Messfläche erstellten Abfolge von Horizontalschnitten. Die graue Fläche repräsentiert das Laufzeitfenster der jeweiligen Zeitscheibe, ansteigend von $1 \mathrm{~ns}$ auf $4 \mathrm{~ns}$. Angegeben ist jeweils der Basiswert für jede Zeitscheibe, ansteigend von 2 ns bis 16 ns. Es ergeben sich für jede Messfläche 4 Spalten. Die Anzahl der Zeilen ist abhängig von dem bei der Messung erfassten Laufzeitfenster.

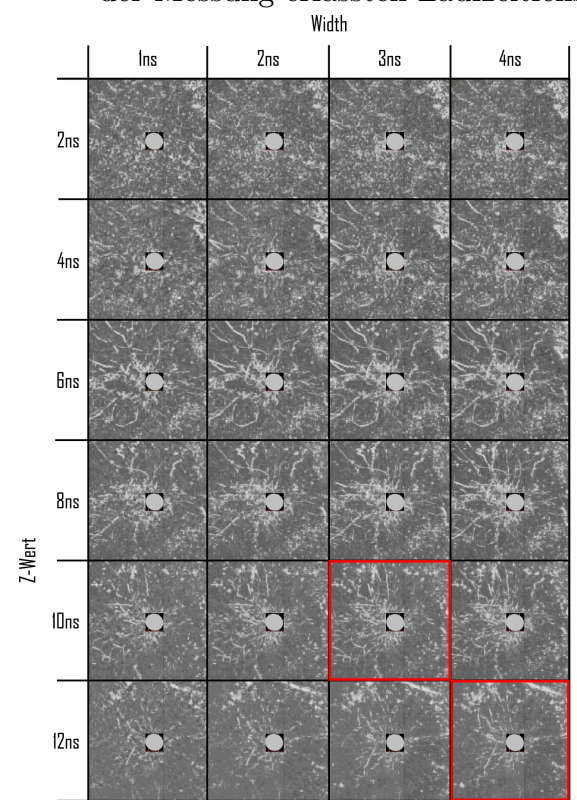

c) Resultierende Gesamtdarstellung der erstellten Zeitscheiben; da ab 12 ns keine Wurzelstrukturen erkennbar sind, wurde auf die Darstellung von $14 \mathrm{~ns}$
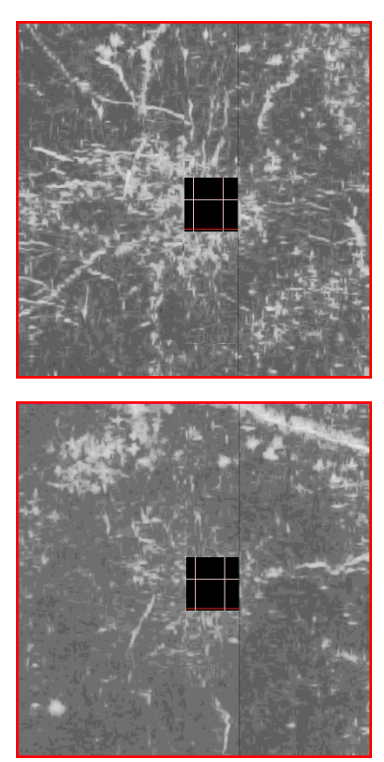

d) Repräsentative Zeitscheiben für die Messfläche

Abbildung 3.9: Auswahl der repräsentativen Zeitscheiben für die jeweilige Messfläche. Aus dem vorliegenden Datensatz werden Zeitscheiben für verschiedene Tiefenstufen mit unterschiedlicher Laufzeit erstellt 3.9a. Die Zeitscheiben werden nach dem in 3.9b und 3.9c dargestellten Schema angeordnet und auf ihren Informationsgehalt bezüglich der Wurzelverläufe auf den Messflächen untersucht. Für den Ergebnisteil werden repräsentative Beispiele (3.9d) ausgewählt; die Gesamtdarstellung (3.9c wird im Anhang gezeigt. 


\subsection{Geoelektrikmessungen}

\subsubsection{Messsystem}

Für die Durchführung der Geoelektrikmessungen wurden die Messapparaturen Geotom (einkanalig) und GeoTom MK RES/IP/SP (mehrkanalig) der Firma Geolog 2000 eingesetzt. Der Vorteil mehrkanaliger Geräte besteht darin, dass zeitgleich mehr als eine Elektrodenkonfiguration gemessen werden kann. Dementsprechend wird die gleiche Anzahl Messwerte innerhalb einer kürzeren Zeitspanne ermittelt. Beide Geräte verfügen über Anschlüsse für zwei Cannon-ITT-Stecker zur

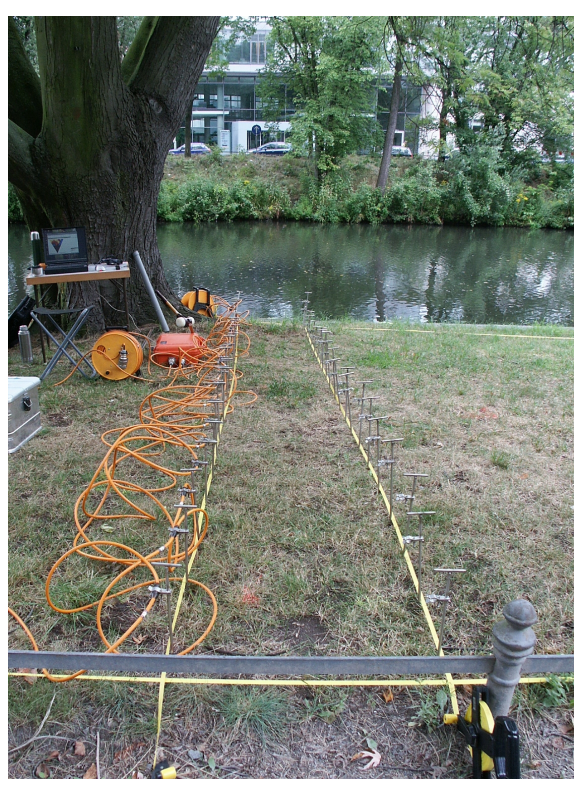

a) Messaufbau Geoelektrik

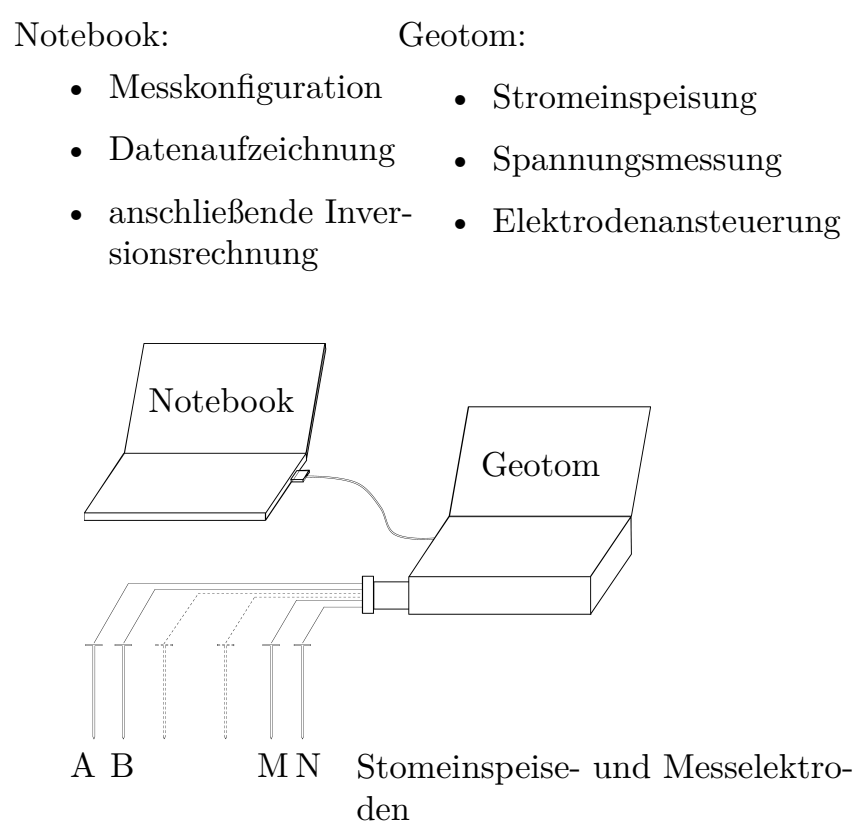

b) Schema Geoelektrik

Abbildung 3.10: Messaufbau bei den durchgeführten Geoelektrikuntersuchungen.

Verbindung des Messgeräts mit mehradrigen Kabelsätzen. Die einkanalige Messapparatur verfügt zusätzlich über Anschlussmöglichkeiten für zwei D-Sub25- Stecker. Die angeschlossenen Kabelsätze werden mit speziellen Edelstahlelektroden verbunden, über die die Einspeisung des Stroms in den Boden und die Messung der entstehenden Spannungsdifferenzen erfolgt. Die Steuerung des Geräts erfolgt mit der Messsoftware GeoTom 7.2 über ein angeschlossenes Notebook. Dadurch werden verschiedene Messkonfigurationen (Stromeinspeise- und Spannungsabgriffspunkte) realisiert. Der Messaufbau ist in den Abbildungen 3.10a und 3.10b dargestellt.

\subsubsection{Durchführung der Messungen}

Allen in diesem Kapitel vorgestellten Kapiteln liegt als Messkonfiguration eine Dipol-DipolAnordnung (vgl. Kapitel 2.3.3 zugrunde. Die Dipol-Dipol-Konfiguration wurde aufgrund der hohen horizontalen Auflösung gewählt. Außerdem haben Rossi et al. (2010) gute Ergebnisse 
mit dieser Konfiguration erzielt. Bis auf einige über die eigentlichen Messflächen hinausragende Auslagen, die auf eine größere Sondierungstiefe abzielten, wurden alle Messungen flächig durchgeführt. Dabei wurden allerdings ähnlich wie beim Einsatz des Bodenradars einzelne Transekte erfasst, aus denen dann die dreidimensionalen Daten interpoliert worden sind. Zur Erfassung eines einzelnen Transekts wurden die 50 Mess- und Stromeinspeiseelektroden, die bei einer Geotommessung zur Verfügung stehen, mit konstantem Elektrodenabstand entlang der zu erfassenden Linie angeordnet. In der Regel wurde das Messtransekt nach Abschluss der Messung um den Elektrodenabstand parallel zum vorher gemessenen Transekt weiterversetzt, bis die gesamte Messfläche erfasst worden ist. Bei den Messungen am Einsteinufer in Berlin sind zusätzlich die orthogonal dazu liegenden Transekte mitgemessen worden.

\subsubsection{Datenbearbeitung, Ergebnisdarstellung und Auswertung}

Die Inversion der Rohdaten erfolgte mit den Programmen DC2DInvRes, DC3DInvRes und BERT (Günther und Rücker 2011). Aus den damit erstellten Modellen der Verteilung des spezifischen Widerstands im Bereich der Messflächen wurden mit dem Visualisierungsprogramm Para View einzelne Horizontalschnitte erstellt, um die Veränderungen der Widerstandsverteilung mit zunehmender Bodentiefe nachvollziehen zu können. Die entsprechenden Schnittbilder werden mit den Ergebnissen der Georadarmessungen verglichen. Da die Ergebnisse einer geoelektrischen Inversion nicht eindeutig sind (vgl. Kapitel 2.3.3), wurden für die Gegenüberstellung von Georadar- und Geoelektrikergebnissen die Tomogramme ausgewählt, bei denen partiell eine Kongruenz zwischen hochohmigen Anomalien im Tomogramm und Reflexionen im Radargramm besteht. Es können daher Abweichungen bei der Tiefenangabe für das Radargramm und das Tomogramm vorkommen. Die Tiefenangabe des Radargramms ist dabei eindeutig, da es sich um ein Laufzeitverfahren handelt.

Für die Messflächen Schwarzkiefer und Buchengruppe auf der Schillerwiese stehen zusätzlich Informationen über die Wurzelverteilung im Untergrund aus den Beprobungen und die Bodenfeuchte zum Zeitpunkt der Messungen zur Verfügung (vgl. Kapitel 3.5). Anhand dieser Daten wird ein möglicher statistischer Zusammenhang zwischen Mess- und Beprobungsdaten untersucht (vgl. Kapitel 3.6.). 


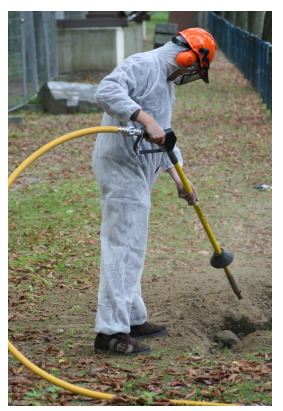

a) Freilegung mit Airspade

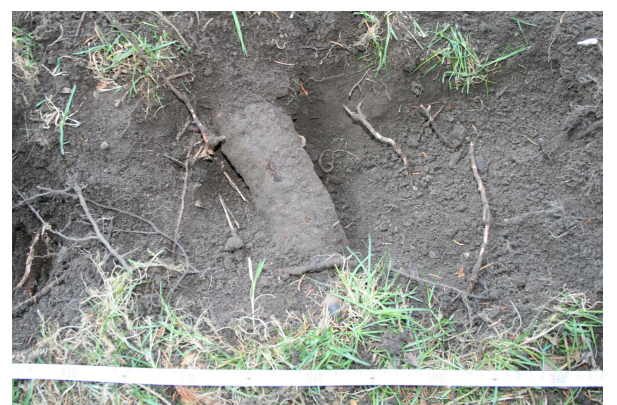

b) Kleinräumige Aufgrabung

Abbildung 3.11: Verifikationsarten

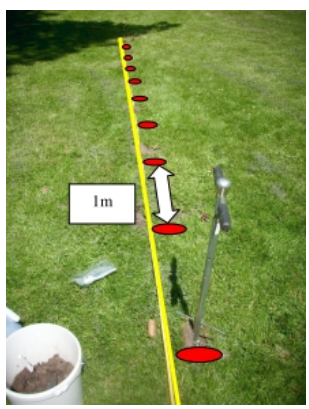

c) Beprobung mit Wurzelbohrer (Foto: Gronwald 2010)

\subsection{Verifikation der Ergebnisse durch Wurzelfreilegungen und Beprobungen}

Großflächige Wurzelfreilegungen fanden auf den Messflächen am Landwehrkanal, in Nimwegen und in Fulda statt (Abbildung 3.11a). Stichprobenhafte Aufgrabungen wurden auf den Messflächen Fichte und Fichtengruppe auf der Schillerwiese durchgeführt (Abbildung 3.11b). Stechzylinderproben wurden auf den Messflächen Schwarzkiefer und Buchengruppe auf der Schillerwiese genommen (Abbildung 3.11c).

Die großflächigen Wurzelfreilegungen wurden mit der Hochdrucklanze Airspade 2000 durchgeführt, durch die die Bodenmatrix um die Wurzeln mit einem Hochgeschwindigkeitsluftstrahl zerstörungsfrei entfernt werden kann. Am Corneliusufer erfolgten Freilegungen und Profilgrabungen, um die Wurzelverhältnisse und die oberflächennahen Untergrundverhältnisse dieser Areale $\mathrm{zu}$ untersuchen.

Für die kleinräumigen Freilegungen wurden Bereiche mit charakteristischen Wurzelverläufen identifiziert und freigelegt, um einen visuellen Vergleich mit der jeweiligen Zeitscheibe aus den Ergebnissen der Radaruntersuchung zu ermöglichen . Die stichprobenartigen Aufgrabungen auf der Schillerwiese erfolgten im Anschluss an die Sichtung der Messergebnisse. Auf der Messfläche Fichte wurden an 38 Stellen im Radargramm identifizierte Wurzeln freigelegt, auf der Messfläche Fichtengruppe an 44 Positionen (Abbildung 3.12a und 3.12b).

Die Wurzelproben auf den Messflächen Schwarzkiefer und Buchengruppe wurden im Zusammenhang mit den Feldarbeiten für die Bachelorarbeit von Gronwald (2010) entnommen. Die entsprechenden Untersuchungen wurden mit einem Wurzelbohrer der Firma Eijkelkamp durchgeführt. Auf beiden Messflächen wurden 48 Proben, verteilt auf jeweils 4 Transekte, genommen (Abbildung 3.12c und 3.12d). Die Ermittlung der Trockenmasse der in den Bodenproben enthaltenen Wurzeln erfolgte durch Auswaschen und Trocknung bei $103^{\circ} \mathrm{C}$ bis zum Erreichen einer Gewichtskonstanz (Gronwald 2010). Zusätzlich wurde auf diesen beiden Messflächen die Bodenfeuchte mit einem Moisture Meter type HH2 der Firma Delta T-Devices bestimmt. 

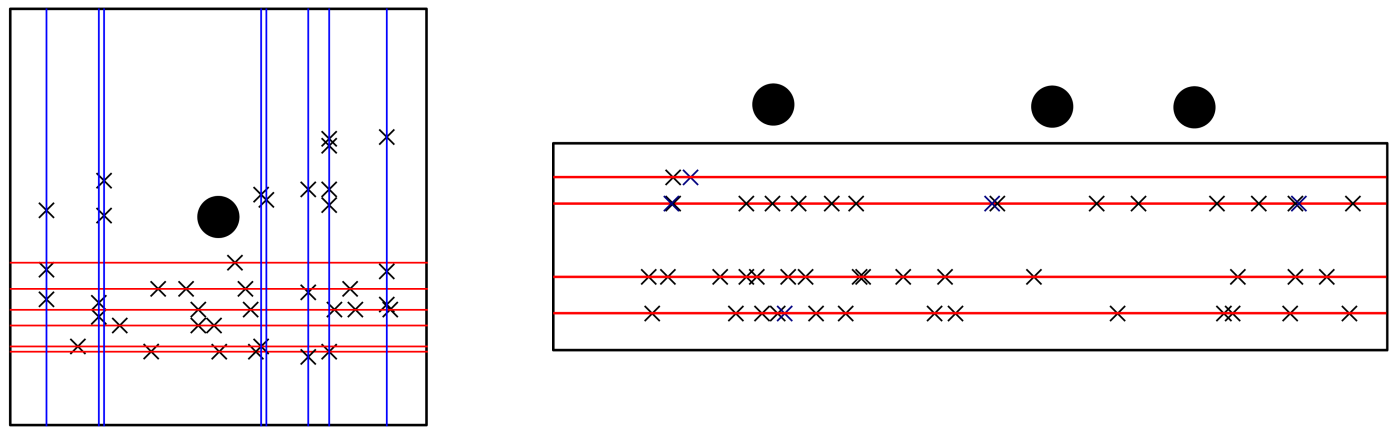

a) Beprobung Fichte

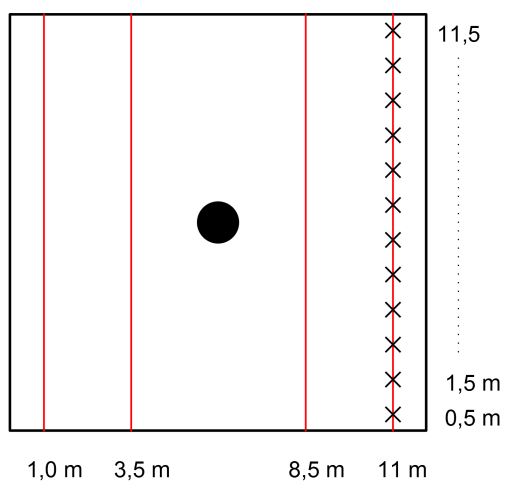

c) Beprobung Schwarzkiefer b) Beprobung Fichtengruppe

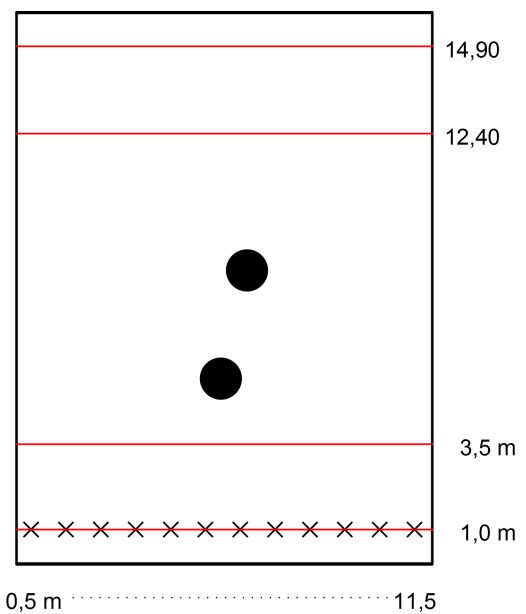

d) Beprobung Buchengruppe

Abbildung 3.12: Lage der Verifikationspunkte auf den Messflächen auf der Schillerwiese: Bei 3.12a und $3.12 \mathrm{~b}$ wurden die Probepunkte so gelegt, dass sie in Bereichen liegen, in denen die Ergebnisse der Bodenradaruntersuchung Wurzeln ausweisen. Bei 3.12c und 3.12d wurde jeweils ein gleichmäßiges Proberaster mit Transekten in 1,0 $\mathrm{m}$ und $3,5 \mathrm{~m}$ Entfernung von den Messflächenrändern und Probepunkten in einem Abstand von je 0,5 m auf den einzelnen Transekten angewendet. Die schwarzen Punkte markieren das jeweilige Baumzentrum. 


\subsection{Statistische Analysen}

Die statistischen Analysen wurden mit der Statistiksoftware R (Team 2012) durchgeführt. Die Zusammenhänge zwischen den ermittelten Widerstandswerten und der Wurzelmasse sowie der Bodenfeuchte wurden mit verallgemeinerten linearen Modellen mit einer logarithmischen Linkfunktion untersucht. Da die räumliche Verteilung der Residuen dieser Modelle systematisch erschien, wurden sie weiter mit verallgemeinerten gemischten Modellen mit Anpassungen für räumliche Korrelationen und Heteroskadisizität analysiert (Zuur et al. 2009).

Der andere Teil der Analysen umfasst den Zusammenhang zwischen gemessenen Laufzeiten und den bekannten Bodentiefen in den Versuchsbecken. 



\section{Ergebnisse}

\subsection{D-Georadarmessungen in den Versuchsbecken}

\subsubsection{D-Radargramme der einzelnen Versuchsbecken}

\subsubsection{Becken 1}

Ausgehend vom südlichen Rand zeichnen sich die ersten sechs Wurzelattrappen in Becken 1 bis zu einem Durchmesser von 3,0 cm als deutliche Diffraktionshyperbeln im Radargramm ab (Abbildung 4.1---). Die Scheitelpunkte dieser Hyperbeln befinden sich dabei jeweils an der Position, an der das Testobjekt liegt, das die jeweilige Hyperbel verursacht. An den Positionen der Attrappen 7, 8 und 9 können keine weiteren Hyperbeln ausgemacht werden. Stattdessen liegt hier eine horizontal verlaufende Reflexion (Abbildung 4.1_ _ ) vor, die wahrscheinlich der Unterkante der Störung im Bodenaufbau entspricht, die durch die Anlage des Versuchs verursacht worden ist. Diese Linie knickt im Bereich des nördlichen Beckenrandes nach oben ab (Abbildung 4.1_ _- ) Dies entspricht wahrscheinlich der Reflexion an der näher kommenden Beckenwand und dem an dieser angebrachten Metallsieb.

Auch die nach Norden zunehmende Tiefe der Oberkanten der Wurzelattrappen lässt sich anhand der später einsetzenden Laufzeiten nachvollziehen (Abbildung 4.1-). Diese steigen von etwa 4 ns -7 ns an.

Auffällig ist ein Unterschied zwischen den Reflexionen an den ersten beiden und den übrigen Positionen. So sind bei Position 1 und 2 jeweils zwei deutlich voneinander abgrenzbare, übereinander liegende Hyperbeln abgebildet (Abbildung 4.1-). Zwischen Position 3 und 6 werden die Wurzelattrappen dagegen mit nur jeweils einer Hyperbel abgebildet.

\subsubsection{Becken 2}

Auch im Radargramm für Becken 2 können an den Positionen der ersten beiden Wurzelattrappen, bei $0,29 \mathrm{~m}$ (1) und $0,88 \mathrm{~m}$ (2), deutliche Diffraktionshyperbeln gefunden werden (Abbildung 4.2 ---). Anstelle weiterer Diffraktionshyperbeln befindet sich zwischen Position 3 und 7 (1,11 m und $1,8 \mathrm{~m}$ ) eine zusammenhängende, horizontal verlaufende Anomalie, deren südliches und nördliches Ende jeweils nach unten abknickt.

An der Position der achten Wurzelattrappe bei etwa 2,1 m im Radargramm befindet sich eine isolierte Hyperbel (Abbildung 4.2-) mit deutlichem Kontrast zum Hintergrund, die früher als die bisher beschriebenen Hyperbeln einsetzt. Das Laufzeitfenster, das diese Hyperbel aufweist, ist größer als bei den anderen Hyperbeln. Weitere früh einsetzende Hyperbeln mit etwas schwächerer Amplitude befinden sich bei den Wurzelattrappen an Position 9 (Abbildung 4.2 ---) und 12 (Abbildung $4.2-$ ). Eine Hyperbel mit schwacher Amplitude, die zu einer späteren Laufzeit 


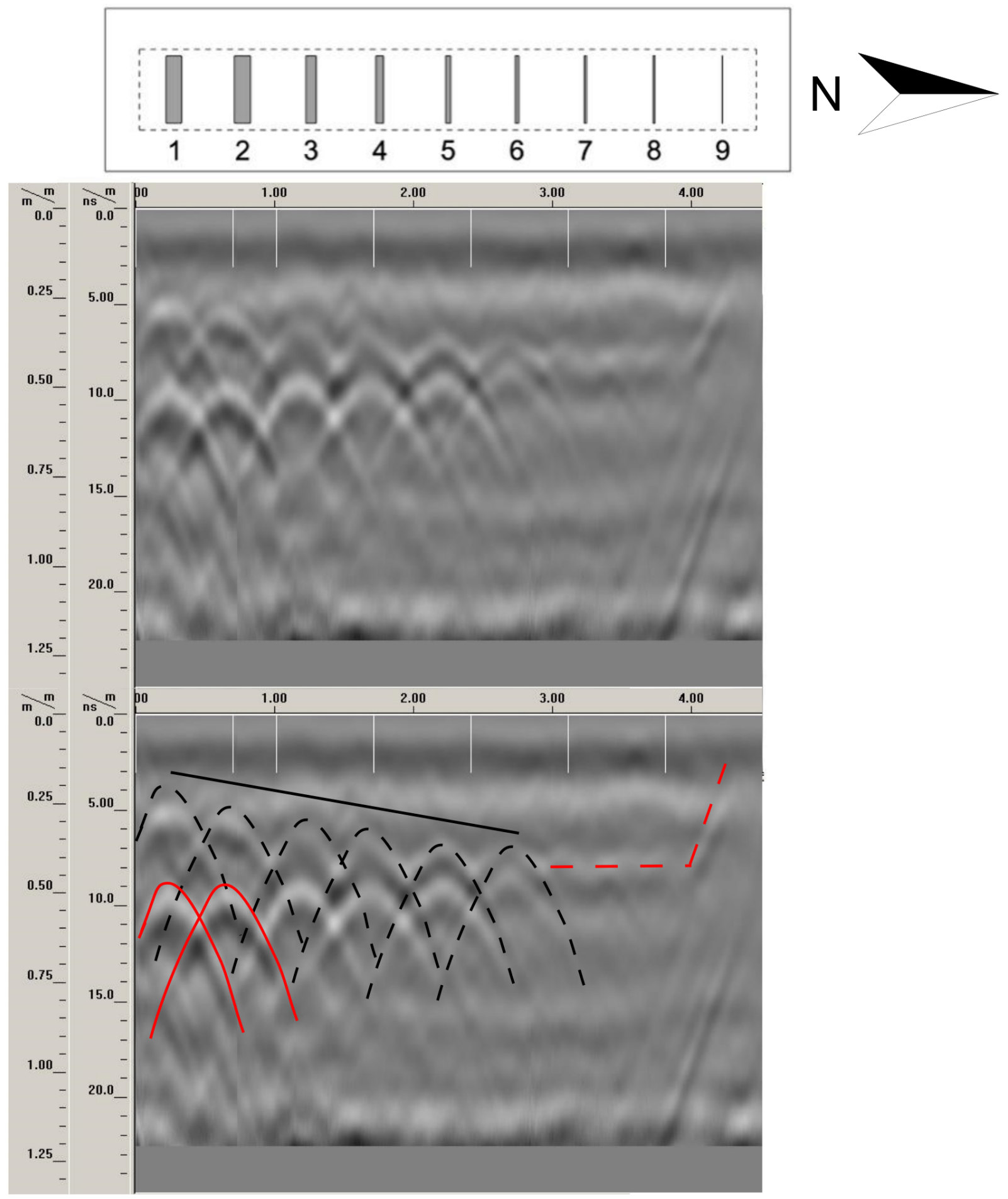

Abbildung 4.1: 2D-Radargramm zu Becken 1 (Tiefenumrechnung mit $v=0,1 \mathrm{~m} \mathrm{~ns}^{-1} ; \varepsilon_{r}=9$ ). 


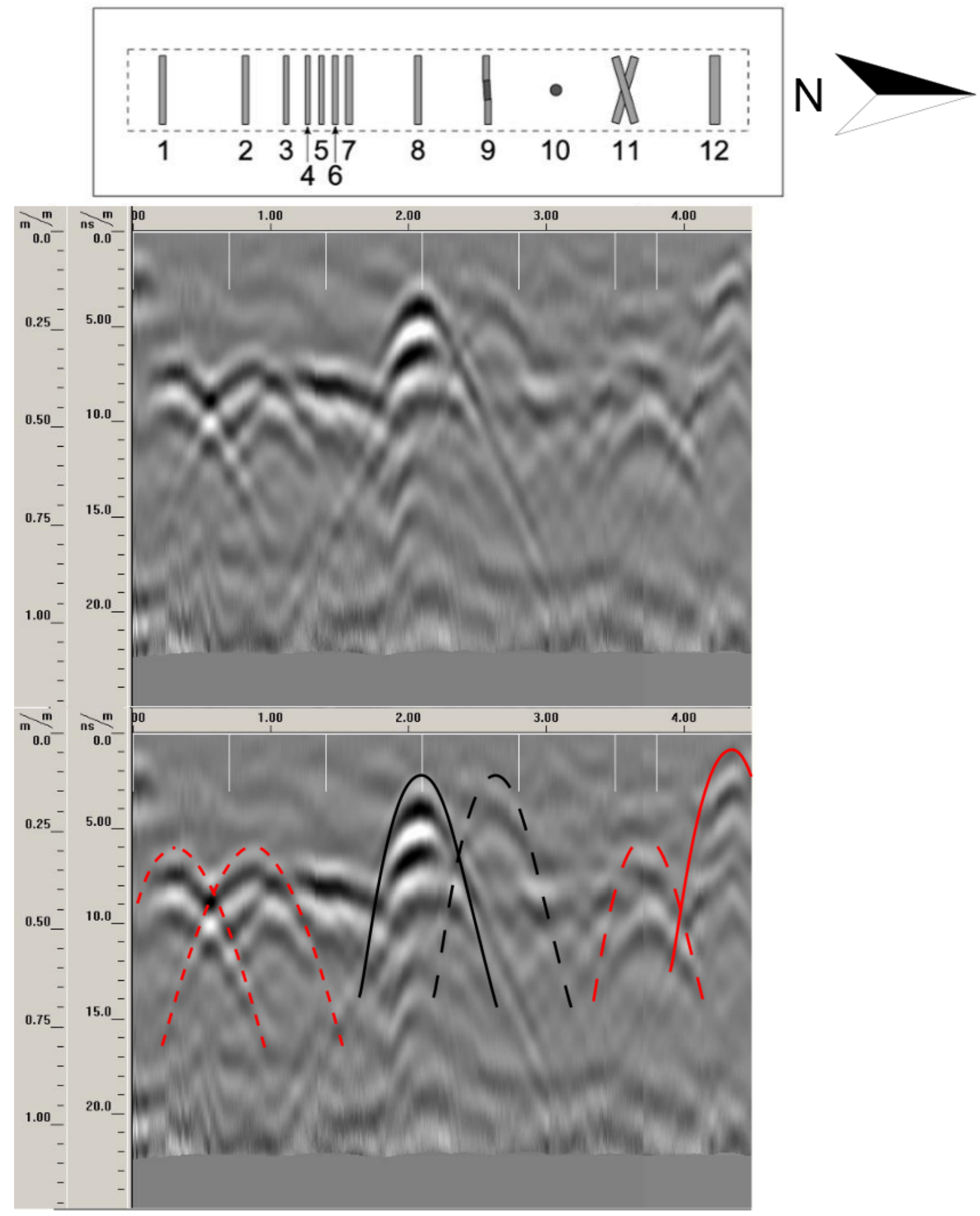

Abbildung 4.2: 2D-Radargramm zu Becken 2 (Tiefenumrechnung mit $v=0,1002 \mathrm{~m} \mathrm{~ns}^{-1} ; \varepsilon_{r}=8,96$ ). 
einsetzt, befindet sich bei Attrappe 11 (Abbildung 4.2 ---). Dagegen liegt bei Wurzelattrappe 10 eine horizontale Anomalie mit relativ schwacher Amplitude vor.

\subsubsection{Becken 3}

Das zu Becken 3 gehörende Radargramm weist im südlichen Teil (im Bereich von Wurzelattrappe 1) eine horizontal verlaufende Anomalie auf. Daran schließt sich bei Position 2 eine deutliche Hyperbel an, deren Ersteinsatz bei etwa 4 ns liegt. Zwischen Position 3 und 5 liegt eine deutliche Reflexion vor (Abbildung 4.3 ---), bei der keine einzelnen Hyperbeln den eingebrachten Prüfkörpern zugeordnet werden können. An den einzelnen Hyperbelästen innerhalb der Reflexion ist allerdings erkennbar, dass die Reflexion verschiedenen Objekten zuzuordnen ist. Diese liegen aber in einer so dichten horizontalen Abfolge vor, dass keine weitere Differenzierung möglich ist.

Bei Position 6, an der sich die einzelnen Steine befinden, liegt eine horizontale Anomalie vor, deren Ränder nicht nach unten abknicken. Im nördlich daran anschließenden Teil des Radargramms befinden sich zwei weitere Hyperbelkomplexe. Der Ersteinsatz dieser Reflexionen liegt im Bereich von etwa 6 ns. In diesem Zeitbereich lassen sich keine einzelnen Hyperbeln finden, allerdings lassen auch hier einzelne Hyperbeläste zu späterer Laufzeit darauf schließen, dass sich die vorliegende Reflexion aus mehreren Einzelreflexionen mit geringem horizontalen Abstand zusammensetzt.

\subsubsection{Becken 4}

Der in Teilen im Radargramm von Becken 1 (Abbildung 4.1) beobachtete Effekt von zwei übereinander liegenden Hyperbeln tritt auch in Becken 4 (Abbildung 4.4 auf. Hier sind bei den ersten 4 (Abbildung 4.4 ---) der insgesamt 7 (Abbildung 4.4--) deutlich zu erkennenden Diffraktionshyperbeln zwei übereinanderliegende Hyperbeln zu beobachten. Die Laufzeit, bei der der Ersteinsatz der jeweiligen Anomalie liegt, nimmt dabei von Position 1 zu Position 4 zu. Zwischen Position 5 und 7 befinden sich drei einzelne Hyperbeln, deren Ersteinsatz von Süd nach Nord früher auftritt. Auffällig ist, dass ein Wechsel beim Vorzeichen der erfassten Amplituden (vgl. Kapitel 2.3.2.1, Gleichung 2.1) zwischen den Attrappen 1 bis 4 und 5 bis 7 auftritt. Die Phasenumkehr, die sich darin zeigt, dass bei den ersten 4 Hyperbeln die Abfolge der Maxima negativ-positiv-negativ (schwarz-weiß-schwarz) ist und die Reihenfolge bei den letzten 3 Hyperbeln positiv-negativ-positiv (weiß-schwarz-weiß), deutet daraufhin, dass die Dielektrizitätskonstanten der verwendeten Wurzelattrappen unterschiedlich sind.

\subsubsection{Ausgewählte wavelets aus den Versuchsbecken}

Die Abbildungen 4.5a bis 4.5c umfassen im rechten Bildteil jeweils ein wavelet aus Becken 1; für die Zuordnung des wavelets zur jeweiligen Aufnahmeposition ist jeweils das Radargramm aus Becken 1 nebenstehend dargestellt.

Abbildung 4.5a zeigt das wavelet, das zwischen Position 7 und 8 bei der Befahrung von Süd nach Nord aufgenommen worden ist. An dieser Stelle befindet sich keine Wurzelattrappe und im zugehörigen Radargramm ist keine Hyperbel abgebildet. Abbildung 4.5b zeigt das wavelet 


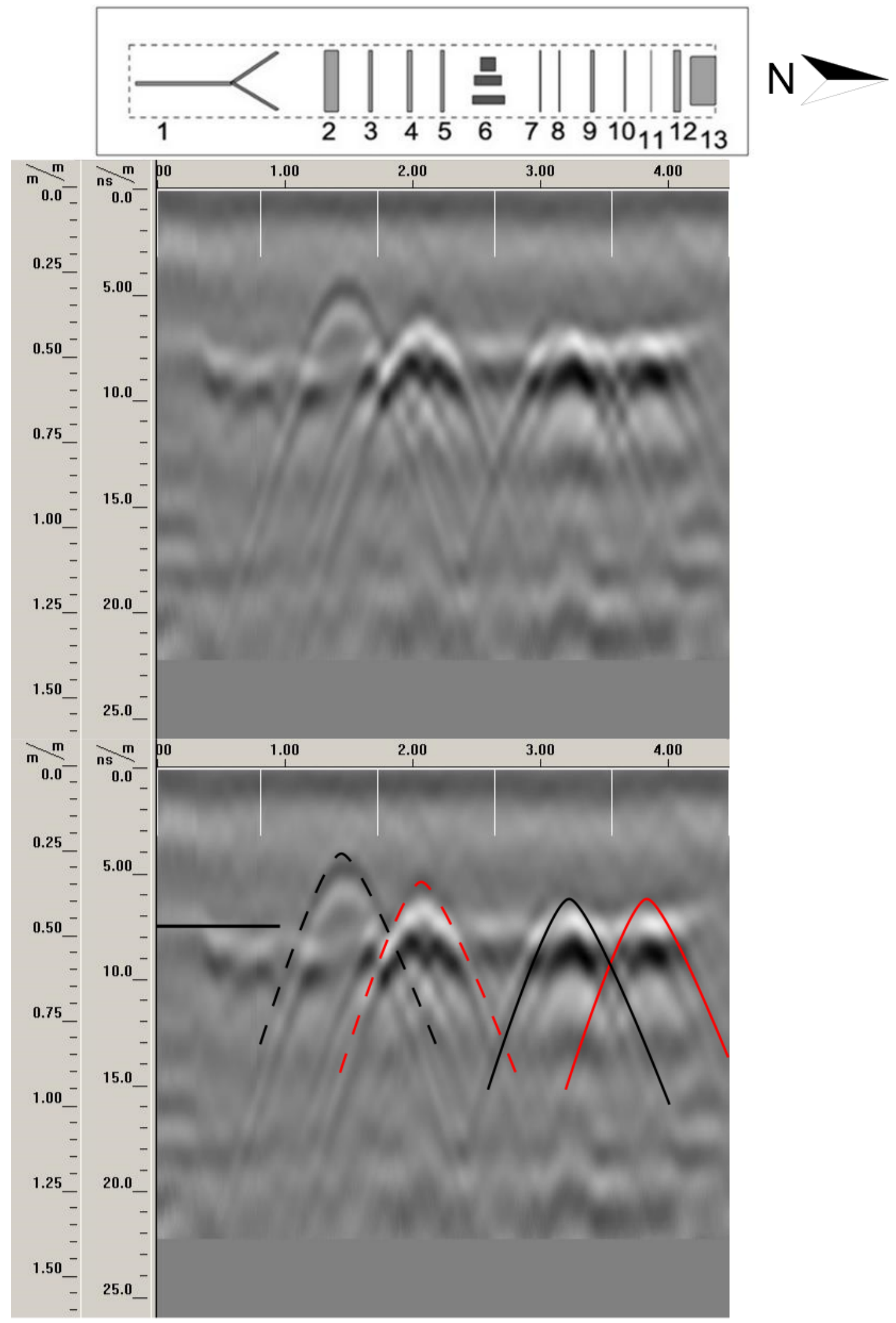

Abbildung 4.3: 2D-Radargramm zu Becken 3 (Tiefenumrechnung mit $v=0,1221 \mathrm{~m} \mathrm{~ns}^{-1} ; \varepsilon_{r}=6,36$ ). 


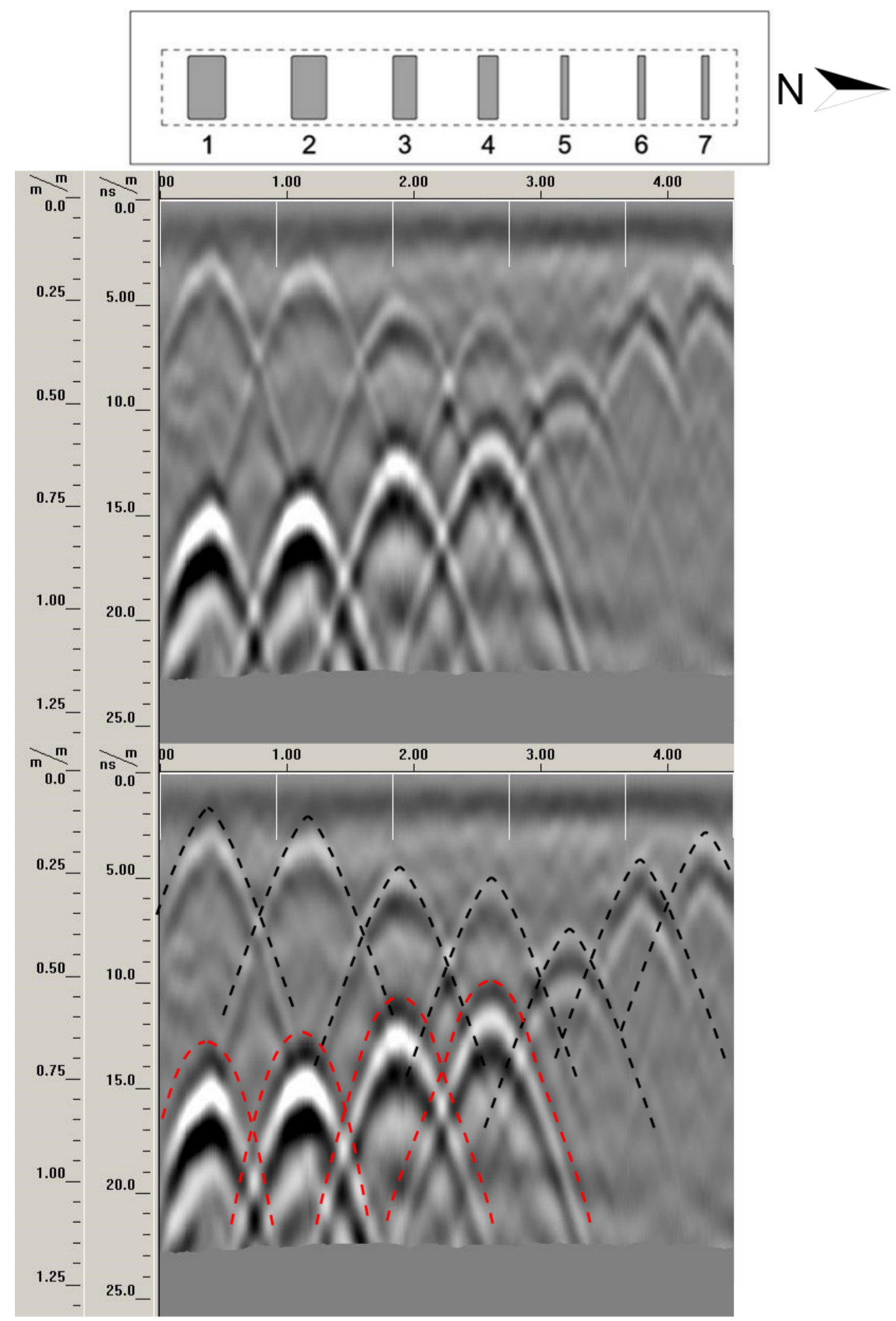

Abbildung 4.4: 2D-Radargramm zu Becken 4 (Tiefenumrechnung mit $v=0,1024 \mathrm{mns}^{-1} ; \varepsilon_{r}=8,59$ ). 


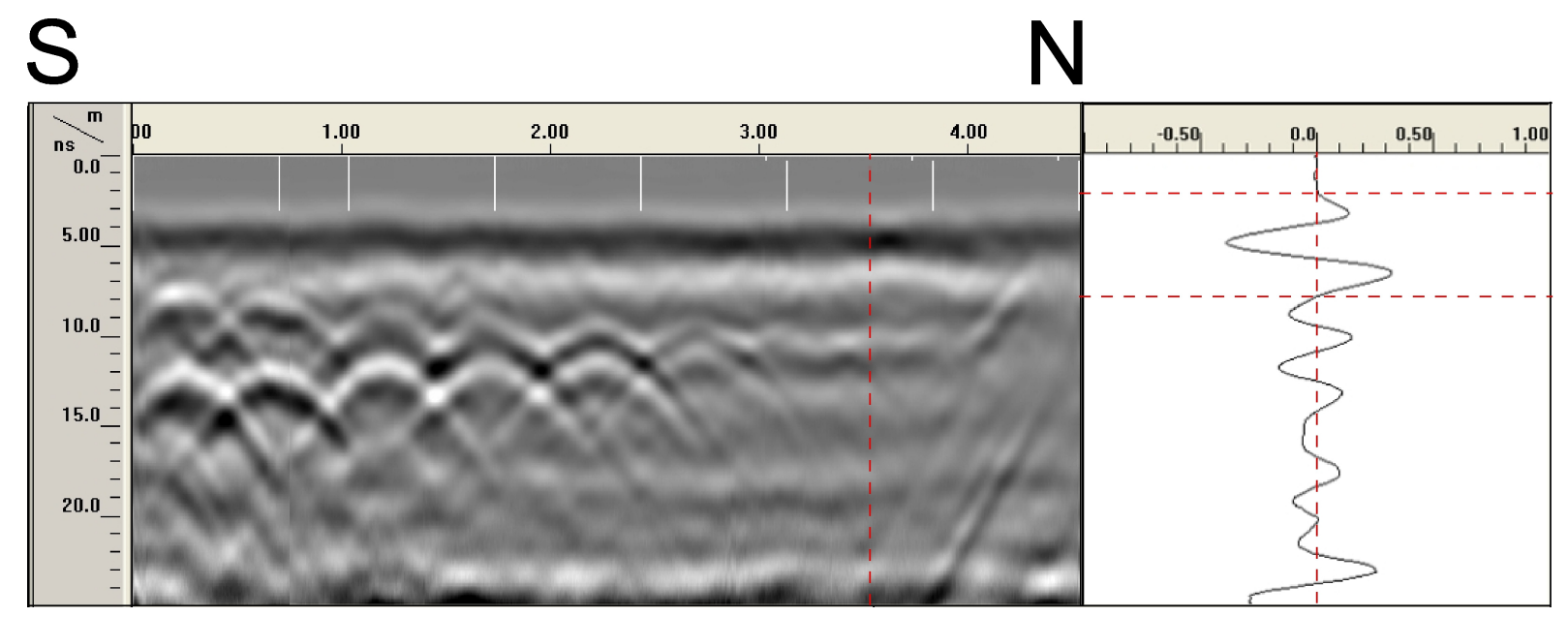

a) wavelet in Becken 1 an Position 3,5 m (ohne Wurzelattrappe und ohne Hyperbel im Radargramm).

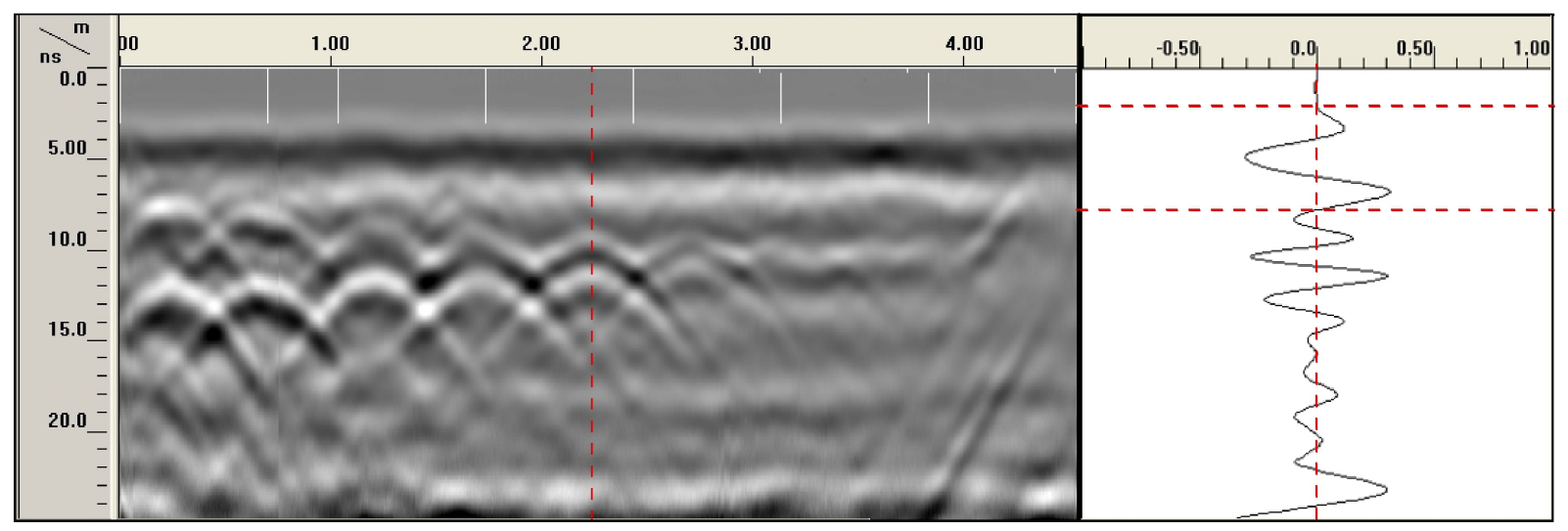

b) wavelet in Becken 1 an Position 2,3 m (mit Wurzelattrappe und einfacher Hyperbel im Radargramm).

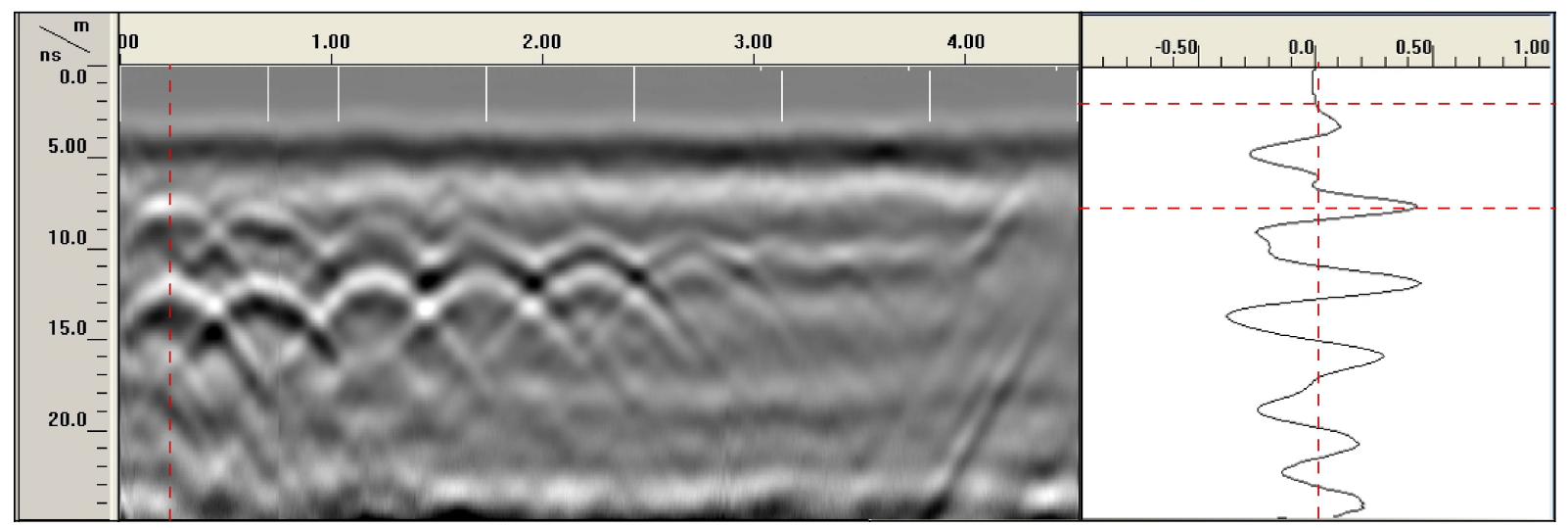

c) wavelet in Becken 1 an Position 0,22 m (mit Wurzelattrappe und übereinanderliegenden Hyperbeln im Radargramm).

Abbildung 4.5: Ausgewählte wavelets aus Becken 1. Zur besseren Identifikation der Bodenreflexion (rechts zwischen _ _ _) wurde keine Laufzeitkorrektur durchgeführt; stattdessen sind unbearbeitete Rohdaten abgebildet. 


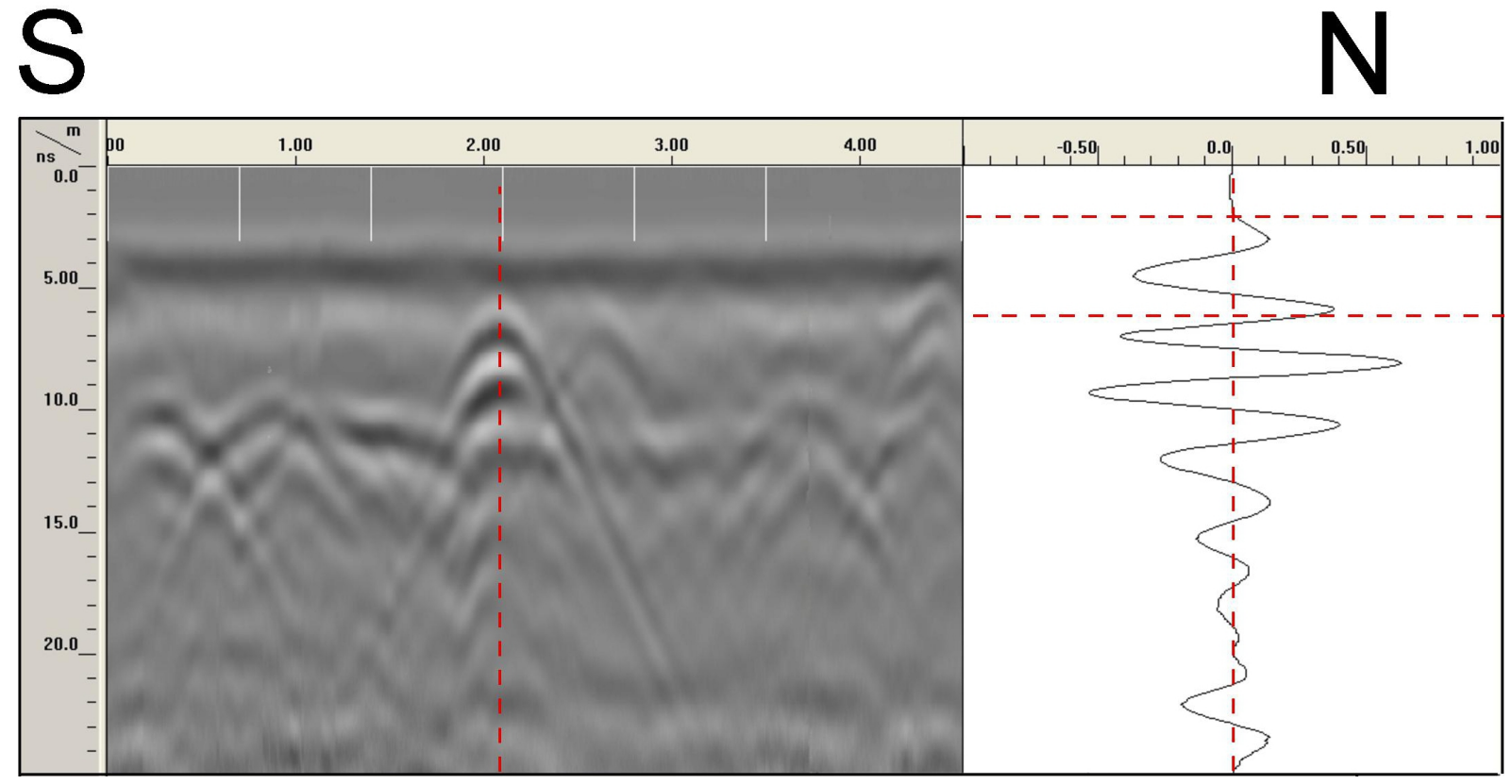

a) wavelet in Lysimeterbecken 2 an Position 2,1 m (mit übereinanderliegenden Wurzelattrappen und Hyperbel mit größerer Vertikalerstreckung im Radargramm).

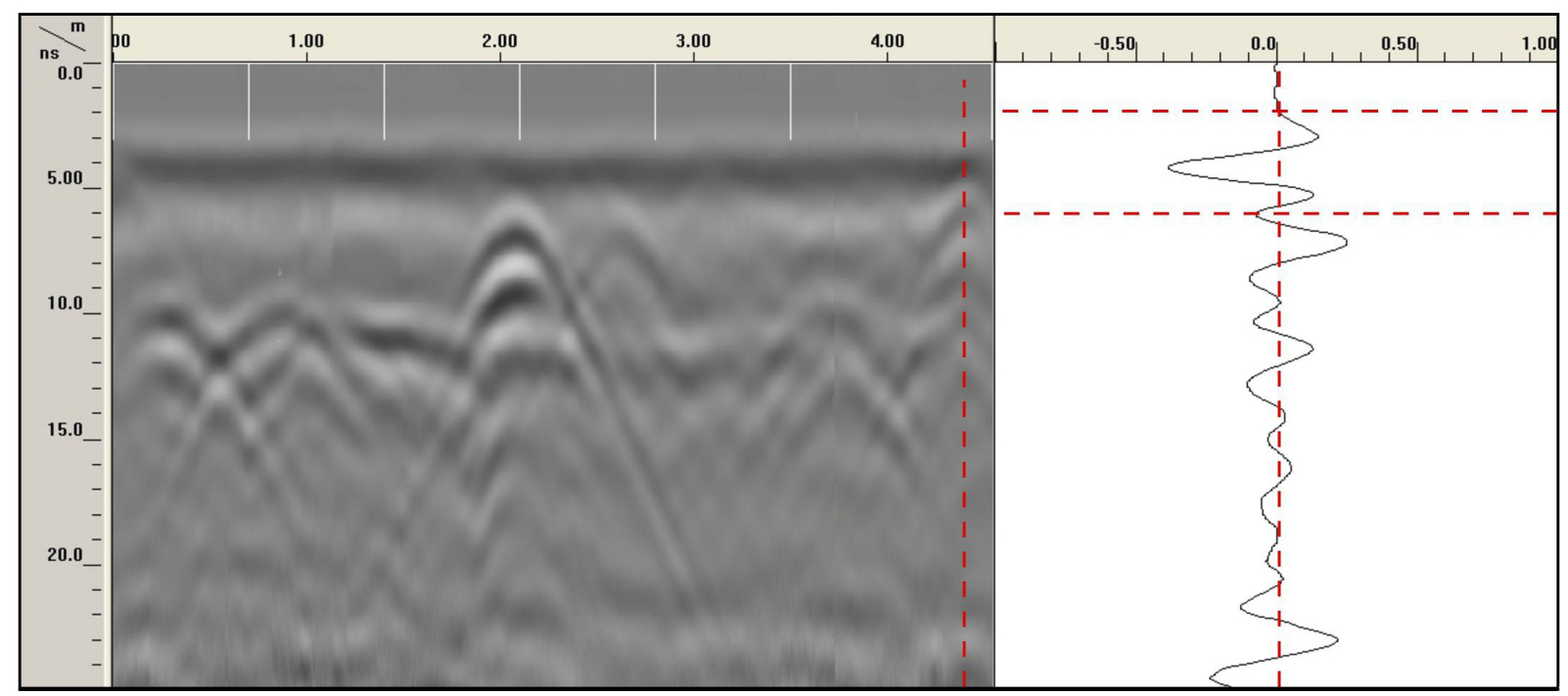

b) wavelet in Lysimeterbecken 2 an Position 4,5 m mit übereinanderliegenden Wurzelattrappen und Hyperbel mit größerer Vertikalerstreckung im Radargramm (mit Wurzelattrappe).

Abbildung 4.6: Ausgewählte wavelets aus Becken 2. Zur besseren Identifikation der Bodenreflexion (rechts zwischen _ _ ) wurde keine Laufzeitkorrektur durchgeführt; stattdessen sind unbearbeitete Rohdaten abgebildet. 
der Befahrung von Süd nach Nord an Position 5 bei etwa 2,3 m. Hier ist im Radargramm eine deutliche Hyperbel abgebildet. Das wavelet in Abbildung 4.5c gehört zu Position (1) bei der Befahrung von Nord nach Süd (etwa $0,22 \mathrm{~m}$ ). Hier befinden sich bei der Darstellung als 2D-Radargramnm die Scheitelpunkte von zwei übereinander liegenden Hyperbeln.

Anhand des wavelets in Abbildung 4.5a lässt sich die Laufzeit der Bodenwelle identifizieren. Damit ist die Breite der Reflexion, die an der Bodenoberfläche stattfindet, gemeint (eingefasst in ---). Die Markierungen (---) in Abbildung 4.5b und Abbildung 4.5c wurden entsprechend der auf diese Weise ermittelten Pulsbreite eingefügt. Es ist erkennbar, dass bei den ersten beiden wavelets eine Differenzierung zwischen der Bodenwelle und den darauffolgenden Reflexionen möglich ist, während im Fall des letzten wavelets die Bodenreflexion und die erste darauf folgende Reflexion ineinander geschoben sind. Folglich treten hier Interferenzen zwischen der Boden- und der Objektreflexion auf.

Abbildung 4.6a zeigt ein weiteres wavelet, das in Becken 2 an Position 8 aufgenommenen worden ist, Abbildung $4.6 \mathrm{~b}$ gehört zu Position 12 in Becken 2. Beide Messanordnungen umfassen übereinander liegende Wurzeln. Ähnlich wie es bei dem wavelet in Becken 1 an Position 1 beobachtet werden kann, lassen sich hier die Bodenreflexion und die jeweils vom eingebrachten Probematerial verursachte Reflexion nicht trennen. Hier liegt ein ausgedehnter Bereich stärkerer Ampltituden vor, bei denen keine Zuordnung zu einzelnen Wurzelattrappen gelingt, da hier Zwischenbereiche mit schwächeren Signalen fehlen. An Position 12 liegt ein ähnlicher Verlauf vor, wobei hier ein insgesamt schwächeres Signal aufgezeichnet worden ist.

\subsubsection{Korrelation von Radarlaufzeiten und eingemessenen Distanzen}

Zwischen den Zwei-Wege-Laufzeiten der eingestrahlten Wellen und der bekannten Tiefenlage der in die Lysimeterbecken eingebrachten Prüfkörper besteht ein ausgeprägter linearer Zusammenhang (Abbildung 4.7). Dabei haben die Ausgleichsgeraden der einzelnen Becken unterschiedliche Steigungen.

In Abbildung 4.7 ist zur Ermittlung der Distanz, die die aufgezeichneten Wellen bei den zweiten Hyperbeln in Becken 1 und 4 zurückgelegt haben müssen, jeweils das Modell einer umlaufenden Welle bzw. Randwelle angenommen worden (vgl. Kapitel 2). Die auf diese Weise ermittelten Laufzeiten fügen sich sehr gut in die Korrelationen der übrigen Laufzeiten und Tiefenlagen für die jeweiligen Becken ein, die als direkte Reflexionen ermittelt worden sind. Im Vergleich zu den Korrelationen der anderen Becken liegt in Becken 3 eine größere Streuung vor.

Ein deutlicher linearer Zusammenhang besteht ebenfalls zwischen den eingemessenen Tiefenlagen der Oberkanten der Wurzelattrappen und den Tiefen der Wurzelattrappen, die aus den GPR-Daten berechnet worden sind (Abbildung 4.8). Wie bei der Korrelation von Laufzeit und Tiefe liegt auch hier bei Becken 3 eine größere Streuung vor. 


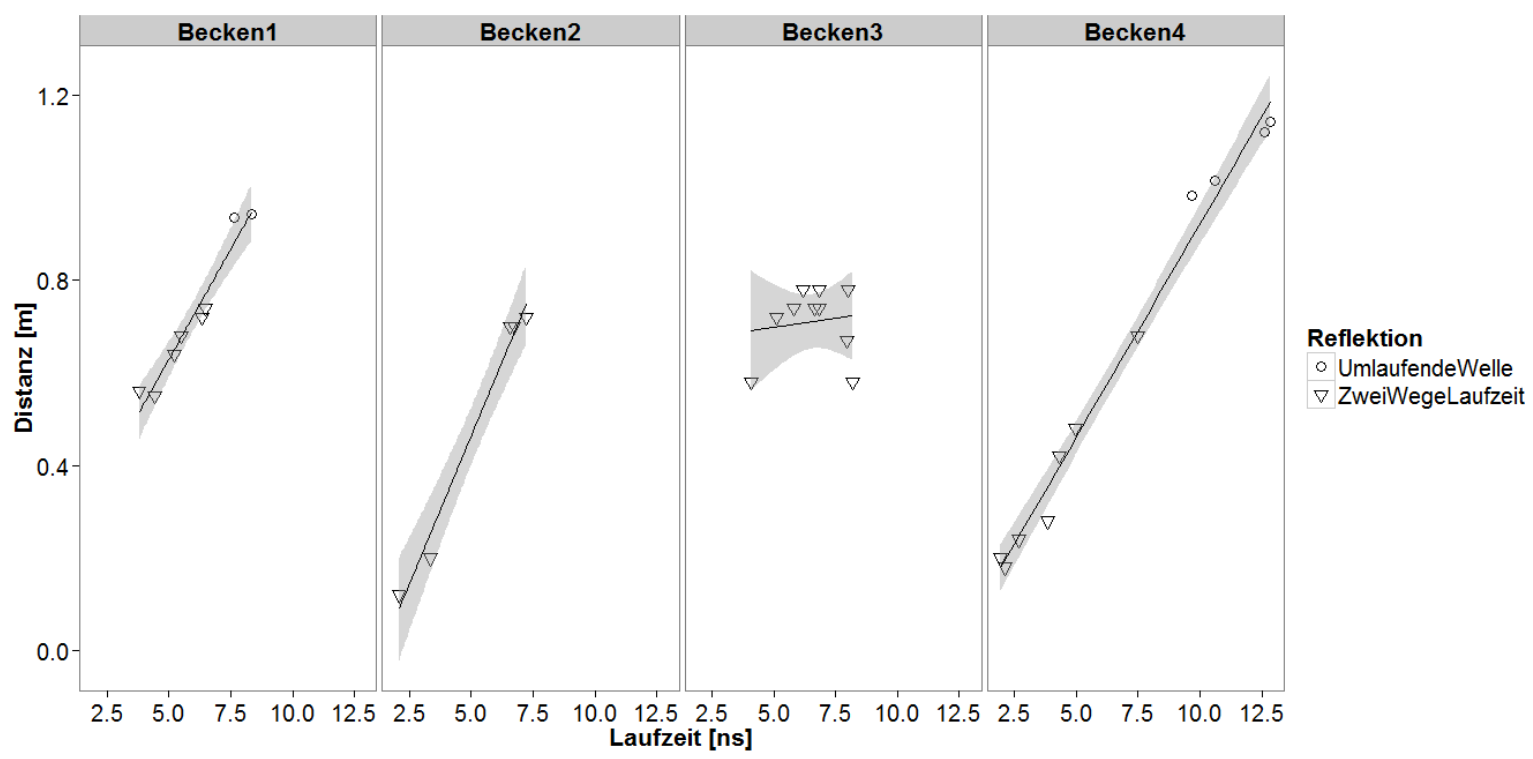

Abbildung 4.7: Korrelation von Reflexionswegen (in m) und Zwei-Wege-Laufzeiten (in ns) in den Versuchsbecken. Bei den meisten Hyperbeln wird eine direkte Reflexion an der Wurzeloberkante für den Reflexionsweg angenommen; bei bestimmten Hyperbeln wird eine Umlaufende Welle als wahrscheinlichster Reflexionsweg angenommen.

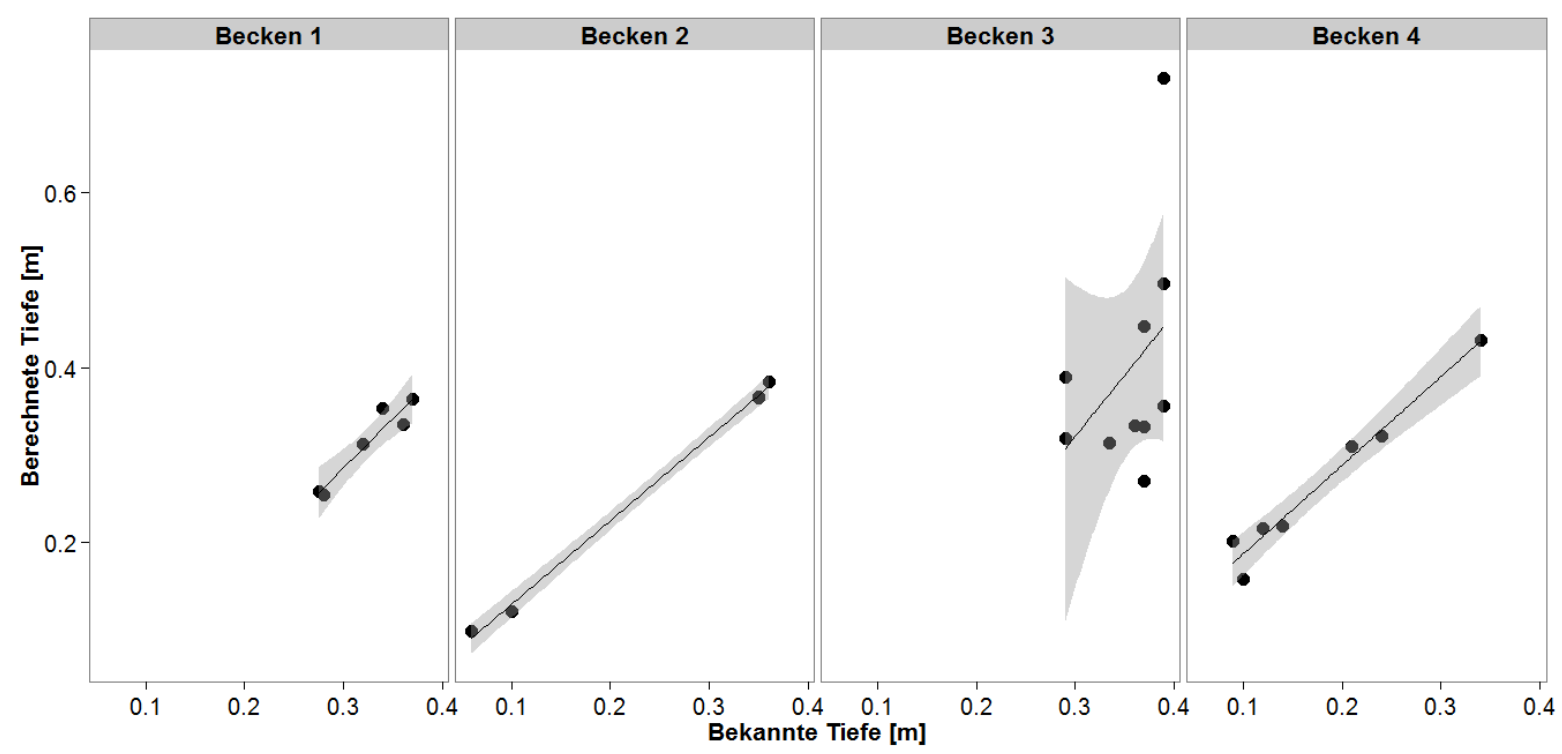

Abbildung 4.8: Korrelation der bekannten Tiefe (in m) der Objektoberkanten mit einer nach Gleichung 2.2 aus den GPR-Daten berechneten Tiefe (in ns). Die zur Berechnung notwendige Geschwindigkeit $v$ wurde anhand der Hybelbreite mit RADAN 6.6 für die einzelnen Hyperbeln ermittelt, für die Zwei-Wege-Laufzeiten wurde die Laufzeit bis zu den Scheitelpunkten der Hyperbeln ermittelt. 


\subsubsection{Zusammenfassung der Ergebnisse zu den Messungen in den Versuchsbecken}

Die Wurzelattrappen in den Versuchsbecken werden in den Radargrammen bis zu einem Durchmesser von 3,0 cm als Diffraktionshyperbeln dargestellt. Wurzeln unterhalb dieses Durchmessers können nicht identifiziert werden. Wurzeln, deren Durchmesser $10 \mathrm{~cm}$ übersteigt, sind mit zwei übereinanderliegenden Hyperbeln im Radargramm erfasst worden. Wurzeln mit einem geringeren horizontalen Abstand als $25 \mathrm{~cm}$ können nicht als getrennt voneinander wahrgenommen werden, sondern werden als zusammenhängende Reflexion, bei der die typische Hyperbelform verloren geht, erfasst. Eine Unterscheidung übereinanderliegender Wurzeln kann in den dargestellten Radargrammen nicht vorgenommen werden, an entsprechenden Positionen liegen Reflexionen mit einer größeren vertikalen Ausdehnung vor. Bei diesen ist aber keine Identifikation einzelner Objekte möglich. Vertikal verlaufende Wurzeln können im 2D-Radargramm nicht identifiziert werden. Bei schräg nach unten abtauchenden Wurzeln wird eine Diffraktionshyperbel mit geringerer Maximalamplitude aufgezeichnet. Die anderen in den Becken verbauten Materialien (vgl. Tabelle A.3 im Anhang) lassen sich nicht anhand charakteristischer Reflexionen von den Wurzelattrappen unterscheiden.

\subsection{D-Georadarmessungen zu grundlegenden Fragestellungen}

\subsubsection{Antennenpolarisation}

Der Einfluss der Antennenpolarisation auf dreidimensionale Georadaruntersuchungen wird anhand einer Messung an einer Platane auf der Schillerwiese (Tabelle 1 Baum Nr. 2e) veranschaulicht. Es wurden Messungen mit drei verschiedenen Antennenanordnungen durchgeführt: in x- und y-Richtung mit quer zur Befahrungsrichtung angeordneter Antenne und in y-Richtung zusätzlich mit längs zur Befahrungsrichtung angeordneter Antenne. Von Abbildung 4.9 bis Abbildung 4.11 symbolisieren $\hat{f}, \#$ und $\Longrightarrow$ jeweils die Antennenanordnung und Bewegungsrichtung der Antenne. Die grauen Kreise markieren die Positionen der Bäume.

Die Abbildungen 4.9 bis 4.11 zeigen jeweils Gegenüberstellungen von Horizontalschnitten und 2D-Radargrammen, die bestimmten Transekten in den Horizontalschnitten zugeordnet werden können.

In Abbildung 4.9a ist im Bereich der gelben Doppellinie keine Wurzel mit parallelem Verlauf zu erkennen. Die 2D-Radargramme in diesem Bereich zeigen ebenfalls keine Indikation für das Vorhandensein einer Wurzel. Im Horizontalschnitt in Abbildung 4.9b, der mit einer um $90^{\circ}$ gedrehten Antenne bei Beibehaltung der Messrichtung aufgezeichnet worden ist, ist dagegen im Bereich der gelben Doppellinie eine Wurzel vorhanden. Diese verläuft annäherend parallel zur Längsseite der Messfläche. In den beiden B-Scans (Abbildung 4.9b unten) befindet sich an der Position der Wurzel jeweils eine längeres, horizontal verlaufendes Objekt (Abbildung $4.9 \mathrm{~b} \bigcirc$ ).

Von der in Abbildung 4.9b identifizierten Wurzel zweigt ein Objekt mit parallelem Verlauf zur Breitseite der Messfläche ab, das sich auch im Horizontalschnitt identifizieren lässt. Dieses Objekt ist ebenfalls in Horizontalschnitt und 2D-Radargramm von Abbildung 4.9a vorhanden.

In Abbildung 4.10a ist die parallel zur Längsseite verlaufende Wurzel ebenfalls vorhanden. Die 


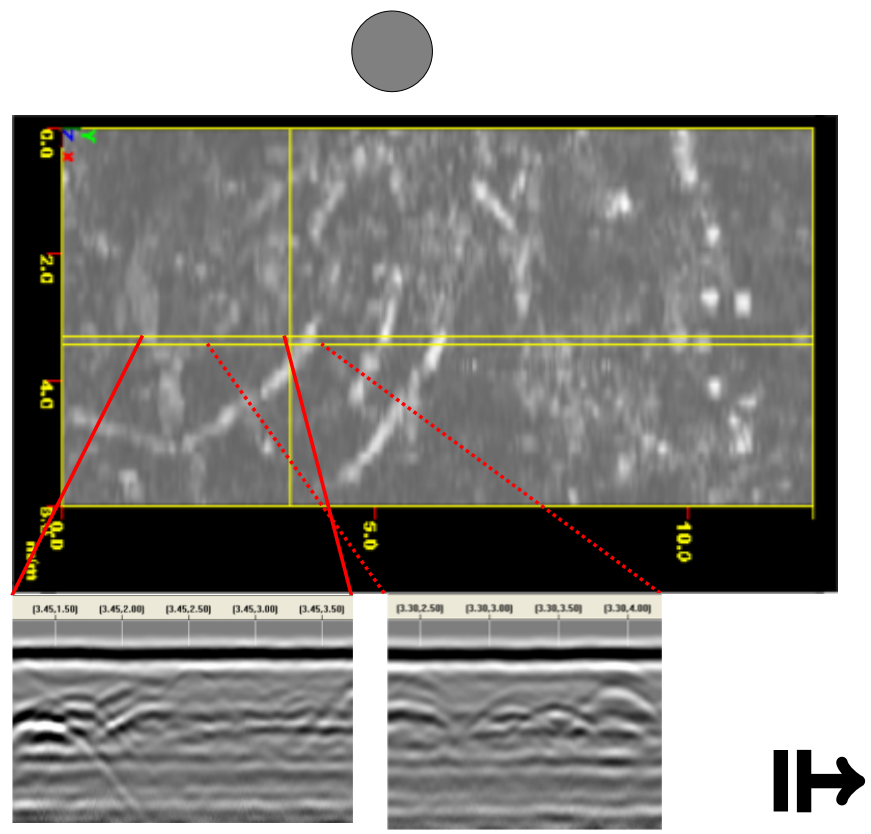

a)

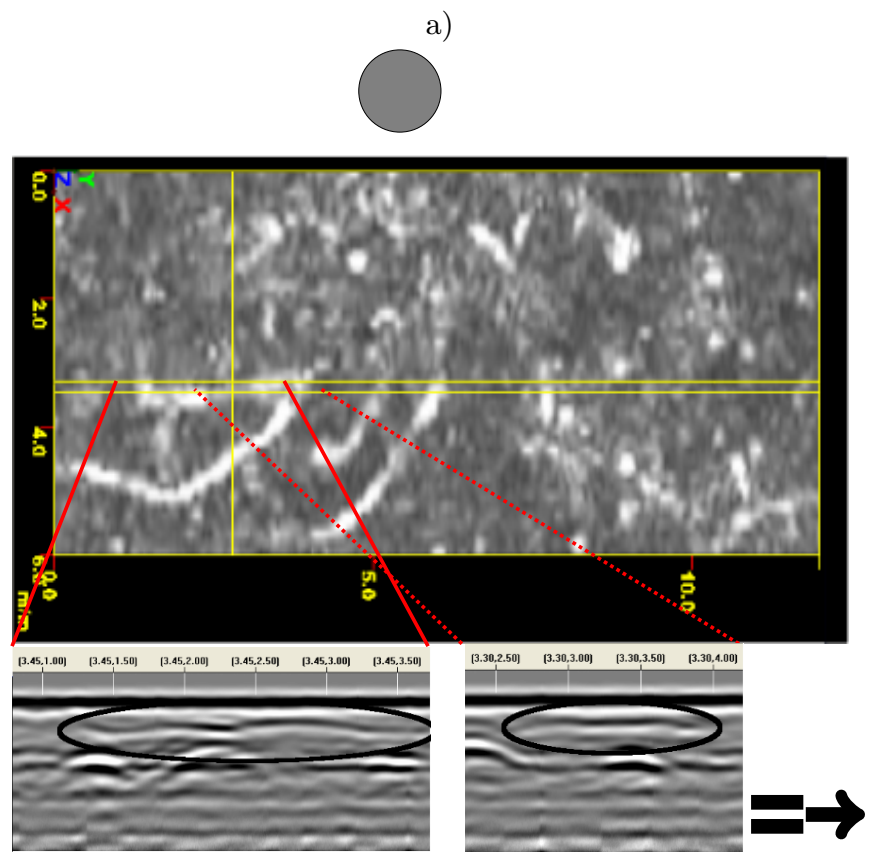

b)

Abbildung 4.9: Darstellung der Messergebnisse einer Bodenradaruntersuchung im Umfeld einer Platane auf der Göttinger Schillerwiese (Messbeispiel 1) in Form eines Vergleichs zwischen B- und C-Scans.

Mit den Parametern z-Wert $=6$; width $=3 ; v=0,07 \mathrm{mns}^{-1} ; \varepsilon_{r}=16$ ergibt sich ein Tiefenbereich von $16,9 \mathrm{~cm}-28,0 \mathrm{~cm}$ für die Horizontalschnitte. Der Pfeil markiert die Messrichtung; die beiden schwarzen Linien die Anordnung der Antennendipole. 


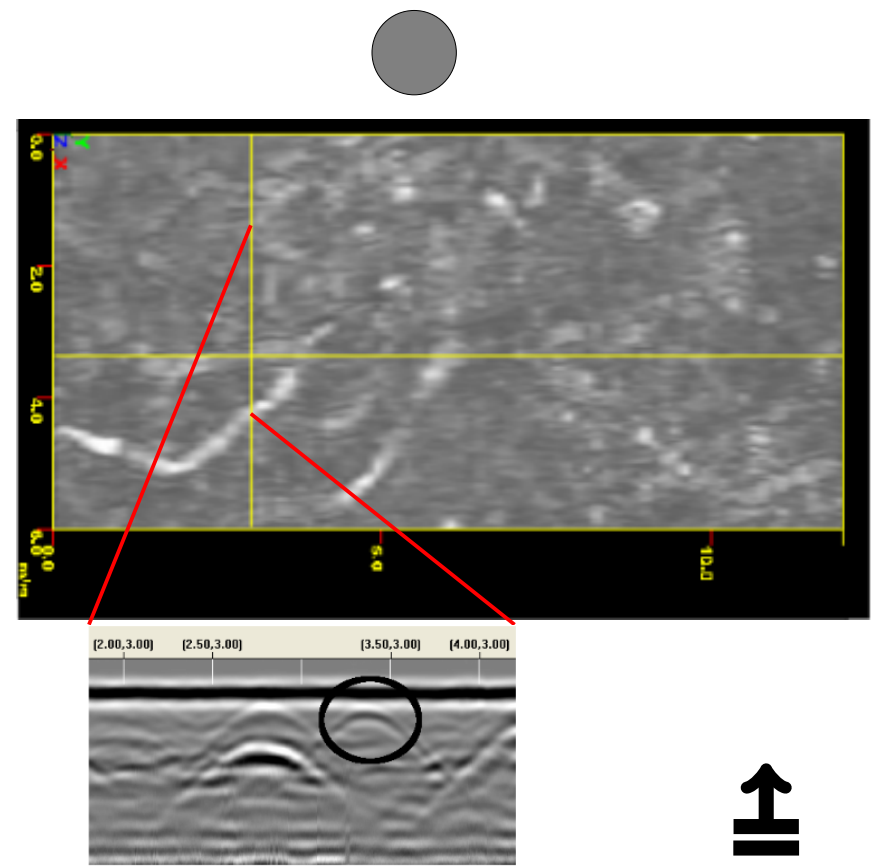

a)

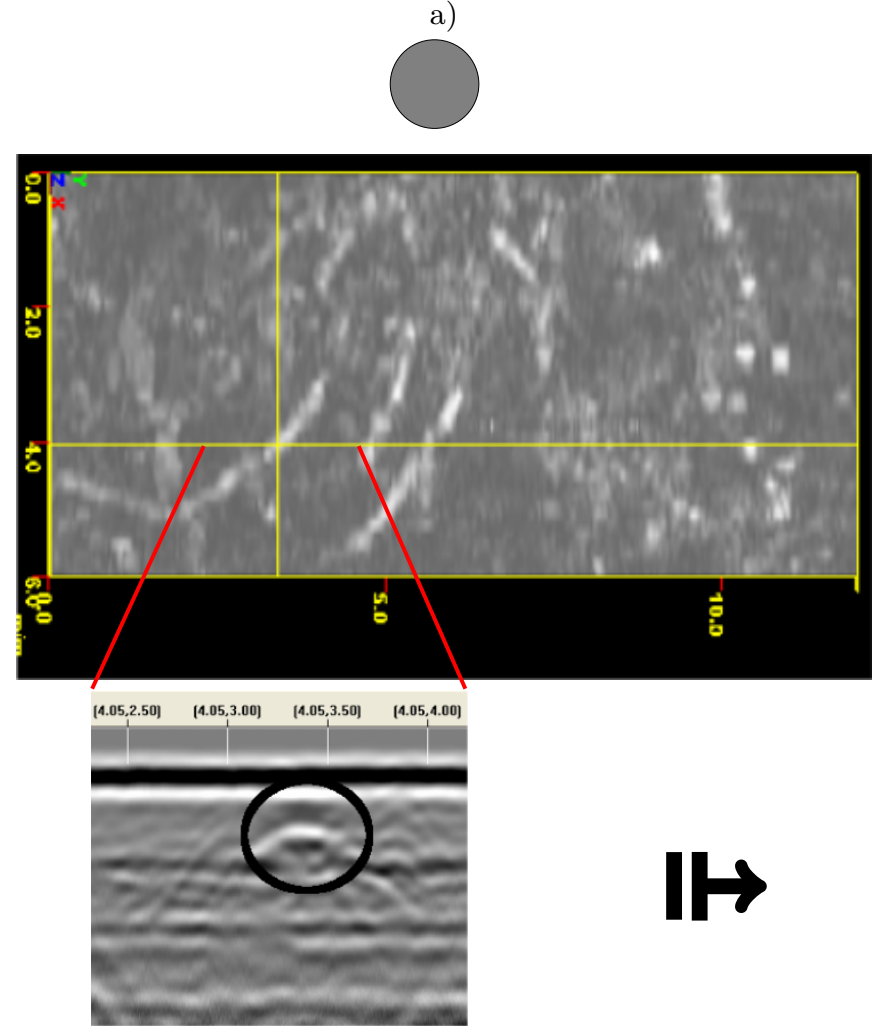

b)

Abbildung 4.10: Darstellung der Messergebnisse einer Bodenradaruntersuchung im Umfeld einer Platane auf der Göttinger Schillerwiese (Messbeispiel 1 und 2) in Form eines Vergleichs zwischen B- und C-Scans.

Mit den Parametern $\mathrm{z}$-Wert $=6$; width $=3 ; v=0,07 \mathrm{mns}^{-1} ; \varepsilon_{r}=16$ ergibt sich ein Tiefenbereich von $16,9 \mathrm{~cm}-28,0 \mathrm{~cm}$ für die Horizontalschnitte. Der Pfeil markiert die Messrichtung; die beiden schwarzen Linien die Anordnung der Antennendipole. 
Amplitude der Wurzel ist in diesem Fall schwächer als in Abbildung 4.9b. Im 2D-Radargramm in Abbildung 4.10a ist an der Position der Wurzel eine deutliche Hyperbel ausgebildet (Abbildung $4.10 \mathrm{a} \bigcirc$ ).

In Abbildung $4.10 \mathrm{~b}$ ist ein weiterer Ast der oben beschriebenen Wurzel zu erkennen, der nach der Vergabelung unter einem Winkel von etwa $45^{\circ}$ in einer etwas größeren Entfernung zur Untersuchungsfläche verläuft. Diese Wurzel ist in den Horizontalschnitten aller drei Antennenanordnungen (Abbildung 4.10b bis 4.11b zu erkennen. Eine entsprechende Anomalie liegt in diesem Fall auch in sämtlichen, zu den verschiedenen Antennenanordnungen gehörenden B-Scans vor (Abbildung 4.10b ?? und 4.11b $\bigcirc$ ). Diese variiert dabei etwas in ihrer Form und Amplitude. Abbildung 4.12 zeigt einen Vergleich der drei Horizontalschnitte, bei dem deutlich wird, dass die in Abbildung 4.12b und 4.12c ersichtlichen Wurzelverläufe fast identisch sind. Allerdings treten in Anordnung 4.12c waagerechte Strukturen auf, die in $4.12 \mathrm{c}$ vollkommen fehlen. Senkrecht verlaufende Strukturen sind dagegen in beiden Abbildungen an identischen Positionen vorhanden, dabei in Anordnung 4.12c aber schärfer abgebildet, während sie in $4.12 \mathrm{~b}$ teilweise etwas verschwommen wirken. In Abbildung 4.12a dominieren im Vergleich zu den anderen Anordnungen waagerechte Strukturen; senkrechte sind nicht vorhanden. Diagonale Strukturen finden sich, wie im Messbeispiel von Abbildung 4.10b bis $4.11 \mathrm{~b}$ beschrieben, dagegen in allen Horizontalschnitten.

Die Synthesen der Ergebnisse von mehreren Antennenanordnungen sind in Abbildung 4.13 in Form von Horizontalschnitten dargestellt. Die wesentlichen Strukturen der Einzelmessungen lassen sich dabei in allen Horizontalschnitten wiederfinden. Allerdings können einzelne Wurzeln in Abbildung 4.13c nicht gefunden werden, obwohl sie in den nebenstehenden Horizontalschnitten vorhanden sind. Im Vergleich mit diesen Abbildungen wirkt die Darstellung der Wurzeln in Abbildung 4.13c auch verschwommen. Die Wurzelverläufe können in Abbildung 4.13b am besten nachvollzogen werden. Insbesondere in der oberen Bildhälfte treten die Wurzeln in diesem Bild deutlich schärfer hervor als in Abbildung 4.13a und 4.13c.

Auf dieser Erkenntnis aufbauend sind die übrigen Messungen in der vorliegenden Arbeit ebenfalls mit den beiden in $4.13 \mathrm{~b}$ genutzten Antennenanordnungen durchgeführt worden. 


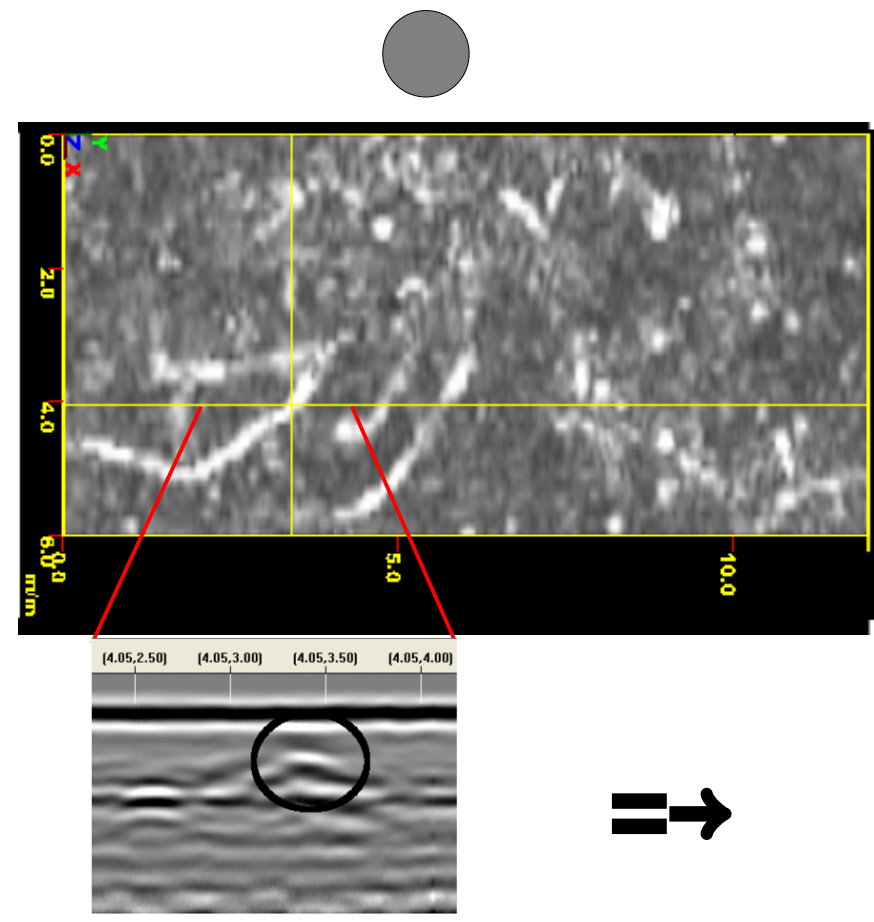

a)

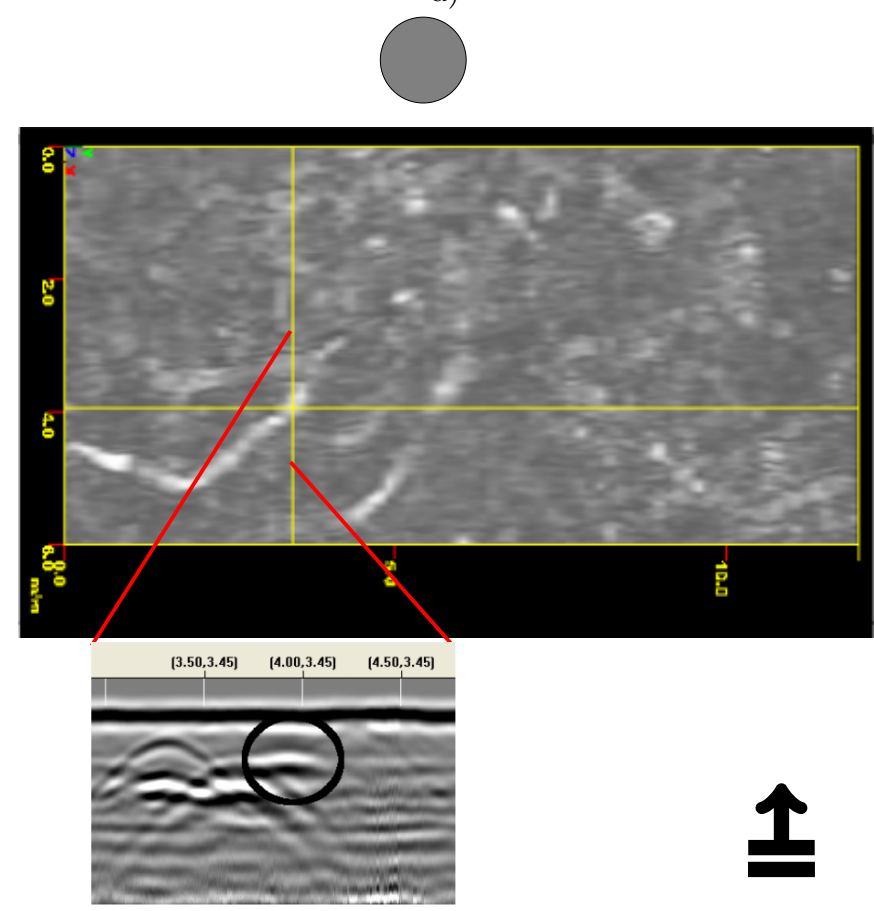

b)

Abbildung 4.11: Darstellung der Messergebnisse einer Bodenradaruntersuchung im Umfeld einer Platane auf der Göttinger Schillerwiese (Messbeispiel 2) in Form eines Vergleichs zwischen Bund C-Scans.

Mit den Parametern z-Wert $=6$; width $=3 ; v=0,07 \mathrm{mns}^{-1} ; \varepsilon_{r}=16$ ergibt sich ein Tiefenbereich von $16,9 \mathrm{~cm}-28,0 \mathrm{~cm}$ für die Horizontalschnitte. Der Pfeil markiert die Messrichtung; die beiden schwarzen Linien die Anordnung der Antennendipole. 


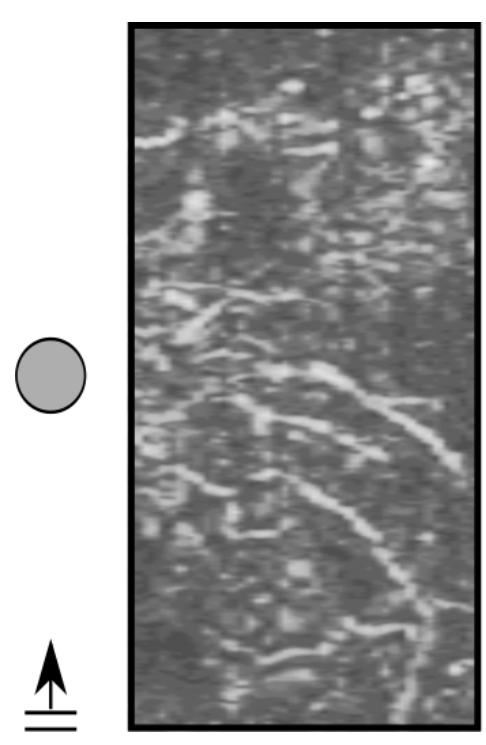

a)

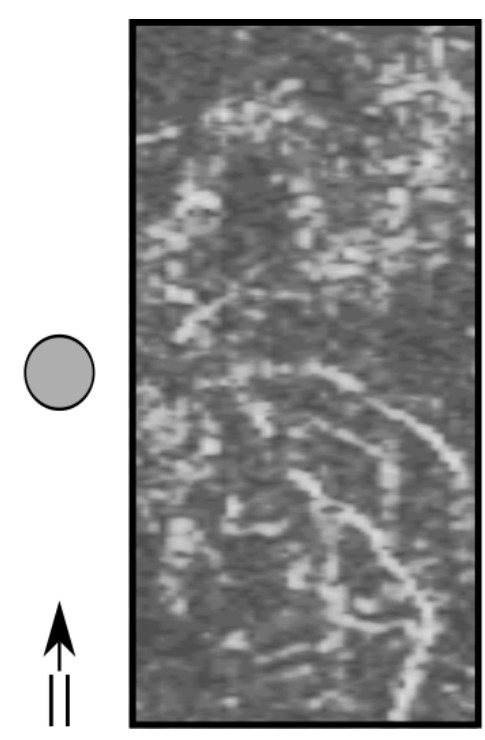

b)

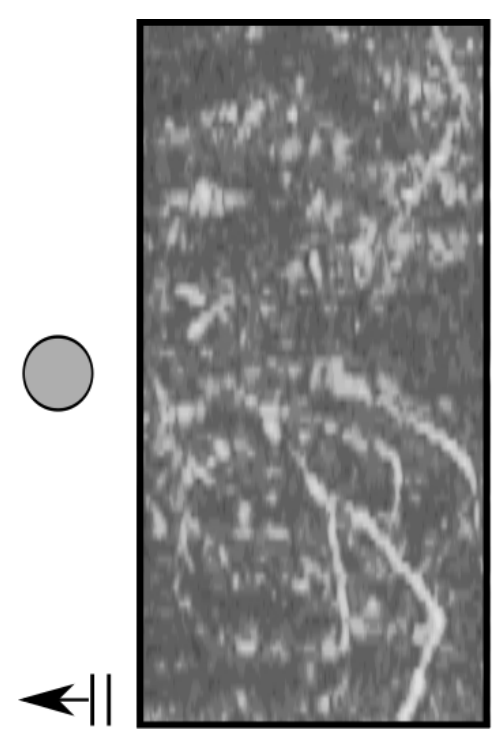

c)

Abbildung 4.12: Darstellung der Messergebnisse einer Bodenradaruntersuchung im Umfeld einer Platane auf der Göttinger Schillerwiese (Messbeispiel 3). Die Messungen erfolgten mit unterschiedlichen Anordnungen der Messantenne zur Untersuchungsfläche und werden in Form von Horizontalschnitten visualisiert. Die dargestellten Horizontalschnitte basieren jeweils auf einer einzelnen Antennenanordnung. Der Pfeil markiert die Messrichtung; die beiden schwarzen Linien die Anordnung der Antennendipole.

Mit den Parametern z-Wert $=6$; width $=3 ; v=0,07 \mathrm{mns}^{-1} ; \varepsilon_{r}=16$ ergibt sich ein Tiefenbereich von $16,9 \mathrm{~cm}-28,0 \mathrm{~cm}$.

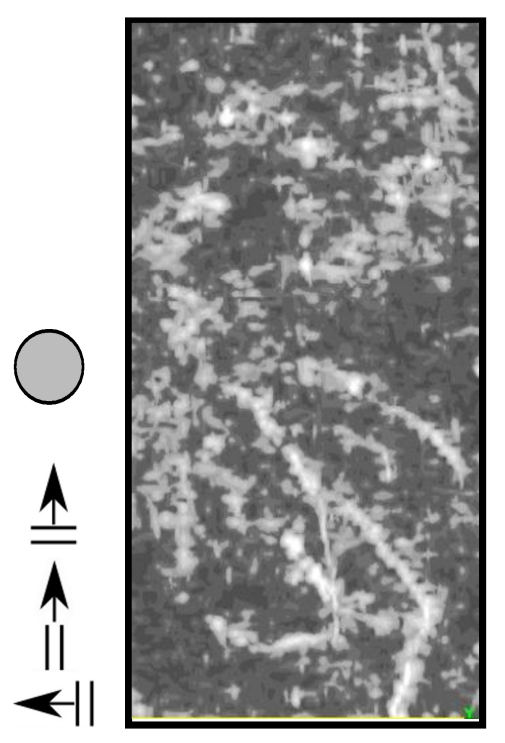

a)

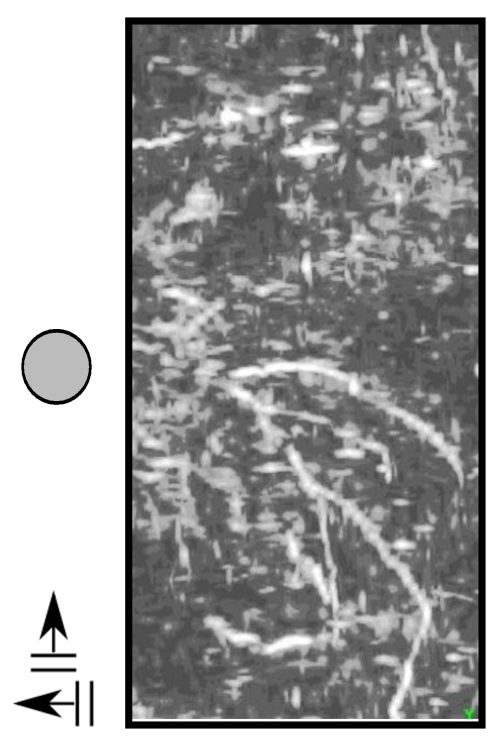

b)

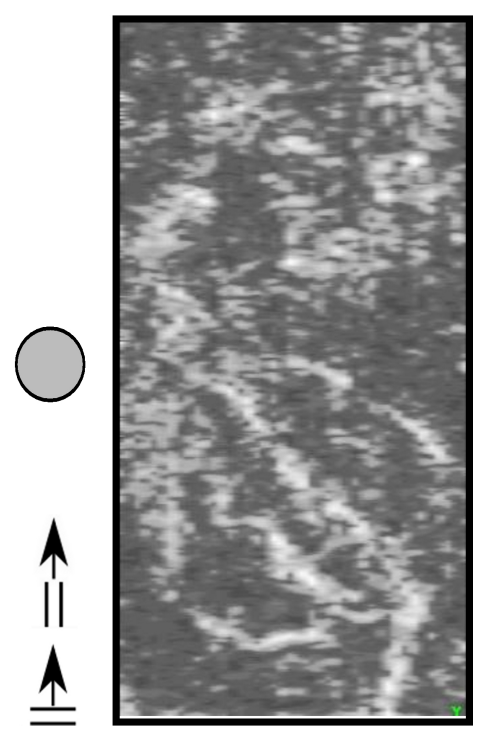

c)

Abbildung 4.13: Darstellung der Messergebnisse einer Bodenradaruntersuchung im Umfeld einer Platane auf der Göttinger Schillerwiese (Messbeispiel 4). Die Messungen erfolgten mit unterschiedlichen Anordnungen der Messantenne zur Untersuchungsfläche und werden in Form von Horizontalschnitten visualisiert. Die dargestellten Horizontalschnitte basieren jeweils auf der Synthese von Messungen mit unterschiedlichen Antennenanordnungen. Der Pfeil markiert die Messrichtung; die beiden schwarzen Linien die Anordnung der Antennendipole. Mit den Parametern z-Wert $=6$; width $=3 ; v=0,07 \mathrm{mns}^{-1} ; \varepsilon_{r}=16$ ergibt sich ein Tiefenbereich von $16,9 \mathrm{~cm}-28,0 \mathrm{~cm}$. 


\subsubsection{Rasterdichte}

Die Abbildungen 4.14a bis 4.14d und 4.15a bis 4.15d zeigen Ergebnisse einer Radaruntersuchung am Standort der Schwarzkiefer auf der Göttinger Schillerwiese (Tabelle 3.1, Baum 2a). Die Abstände der Transekte, die den dargestellten Horizontalschnitten als Grundlage dienen, verringern sich ausgehend von jeweils $60 \mathrm{~cm}$ Abstand bis auf einen Abstand von $15 \mathrm{~cm}$. Während in Abbildung 4.14 die Rohdaten dargestellt sind, wurden die Daten in Abbildung 4.15 mit verschiedenen Filtern (vgl. Kapitel 2.3.2.3 und 3.3.3 bearbeitet, um die Wurzeln als Zielobjekte der Untersuchung deutlicher hervorzuheben. Zur Verdeutlichung der Rasterabstände sind die Positionen der Messtransekte in den Horizontalschnitten als schwarze Linien eingefügt.

Sowohl in Abbildung 4.14 als auch in Abbildung 4.15 ist eine höhere Auflösung der erfassten Wurzeln durch die Verdichtung der Messraster zu verzeichnen. Dabei sind schon beim initialen Rasterabstand von $60 \mathrm{~cm}$ in beiden Abbildungen vermehrte Reflexionen in der Bildmitte zu erkennen, aus der einzelne Strukturen in die Peripherie streichen. Die Ausdehnung und die Erscheinung der zentralen Reflexion bleibt auch bei engerem Rasterabstand annähernd konstant. Veränderungen können dagegen bei den Strukturen ausgemacht werden, die in die Peripherie laufen. So sind in den Abbildungen 4.14a und 4.15a diese Strukturen treppenartig (z.B. jeweils $\longrightarrow$ ) und teilweise nicht zusammenhängend (z.B. jeweils _. - $)$ ) auszumachen. Erst ab einem Messlinienabstand von $30 \mathrm{~cm}$ erscheinen diese Strukturen über längere Distanzen zusammenhängend (Abbildung $4.14 \mathrm{c}$ und $4.15 \mathrm{c} \longrightarrow$ ) und lassen die zu erwartenden Formen und Verläufe von Baumwurzeln erkennen. Des Weiteren führen ab diesem Rasterabstand auch kleinräumige Richtungswechsel nicht zu einem Bruch oder treppenförmigen Verlauf der Wurzeln (Abbildung $4.14 \mathrm{c}$ und $4.15 \mathrm{c},-\rightarrow$ ).

Zwischen den Rohdaten und den mit Filtern (vgl. Kapitel 2.3.2.3 und 3.3.3 bearbeiteten Horizontalschnitten bestehen keine großen Unterschiede. In den bearbeiteten Daten sind die erfassten linearen Objekte etwas schmaler und die Intensität der Reflexionen ist geringer. 


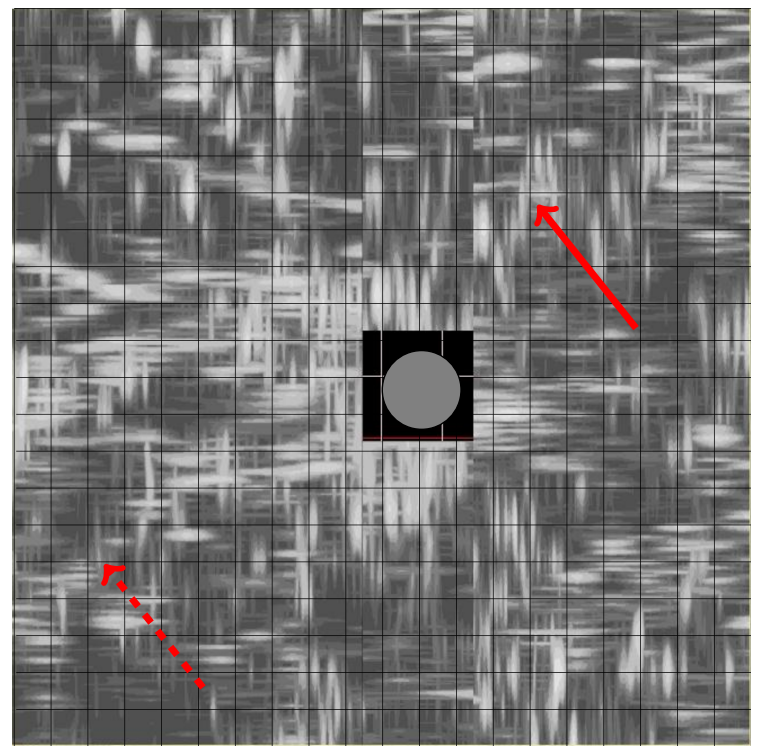

a) $60 \mathrm{~cm}$

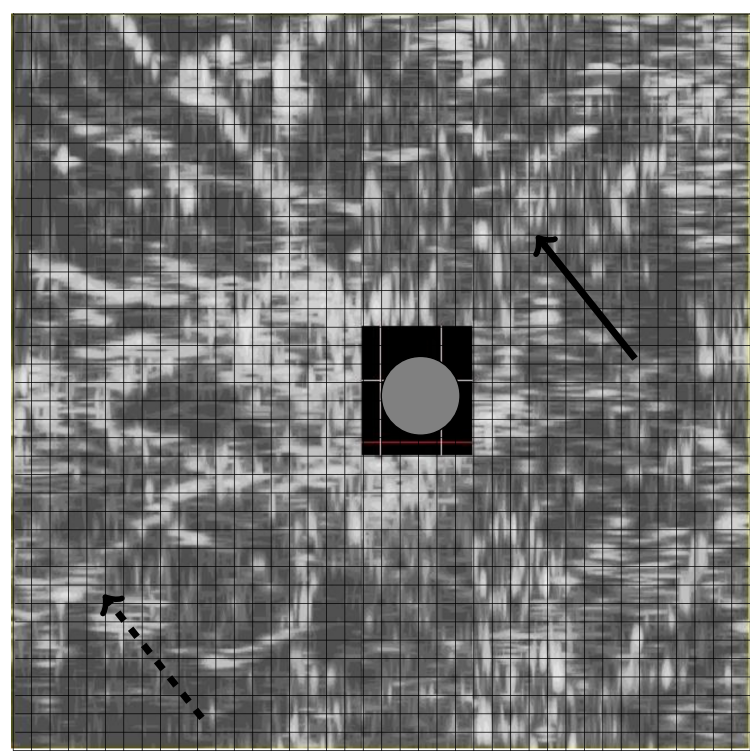

c) $30 \mathrm{~cm}$

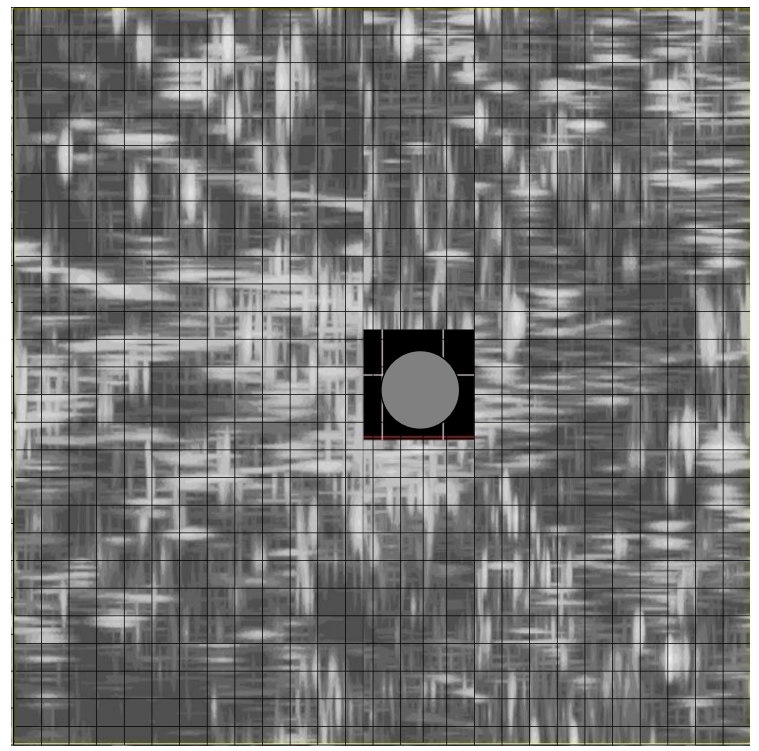

b) $45 \mathrm{~cm}$

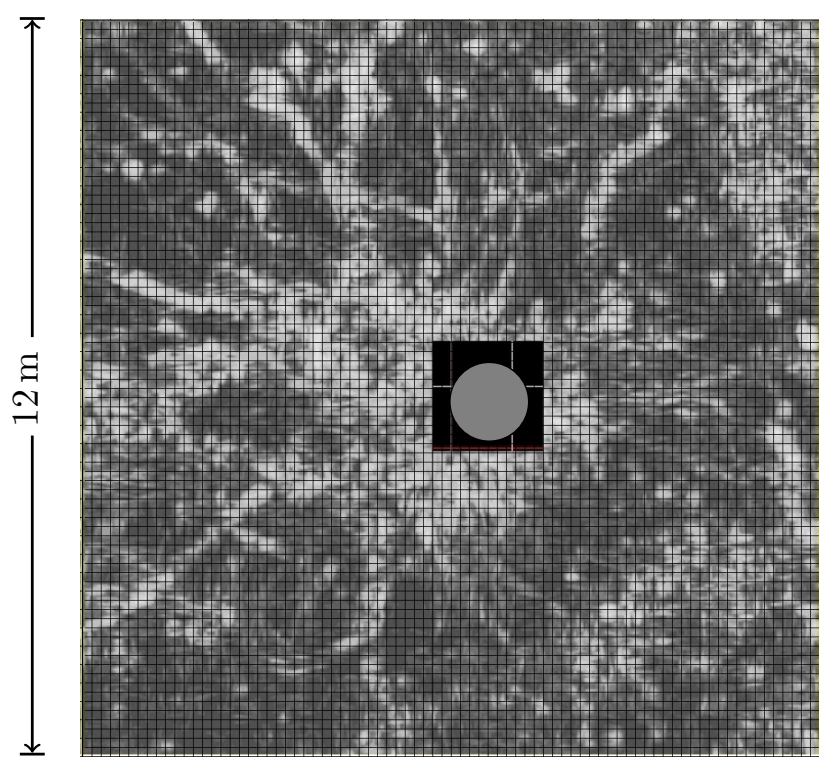

d) $15 \mathrm{~cm}$

Abbildung 4.14: Rasterabstände bei 3D-Georadarmessungen (Rohdaten): Darstellung der Messergebnisse einer Georadarmessung an einer Schwarzkiefer auf der Göttinger Schillerwiese. Die Ergebnisdarstellung erfolgt in Form von Horizontalschnitten der unbearbeiteten Rohdaten. Grundlage der Horontalschnitte ist der gleiche Datensatz. Die Abstände der Transekte, auf denen die 3D-Darstellung basiert, werden von $60 \mathrm{~cm}$ in 4.14a bis $15 \mathrm{~cm}$ in 4.14d jeweils um $15 \mathrm{~cm}$ verringert. Der Baum befindet sich jeweils im Bereich der schwarzen Fläche, die nicht befahren werden konnte.

Mit den Parametern z-Wert $=9$; width $=3 ; v=0,07 \mathrm{~m} \mathrm{~ns}^{-1} ; \varepsilon_{r}=18,36$ ergibt sich ein Tiefenbereich von $26,25 \mathrm{~cm}-36,75 \mathrm{~cm}$. 


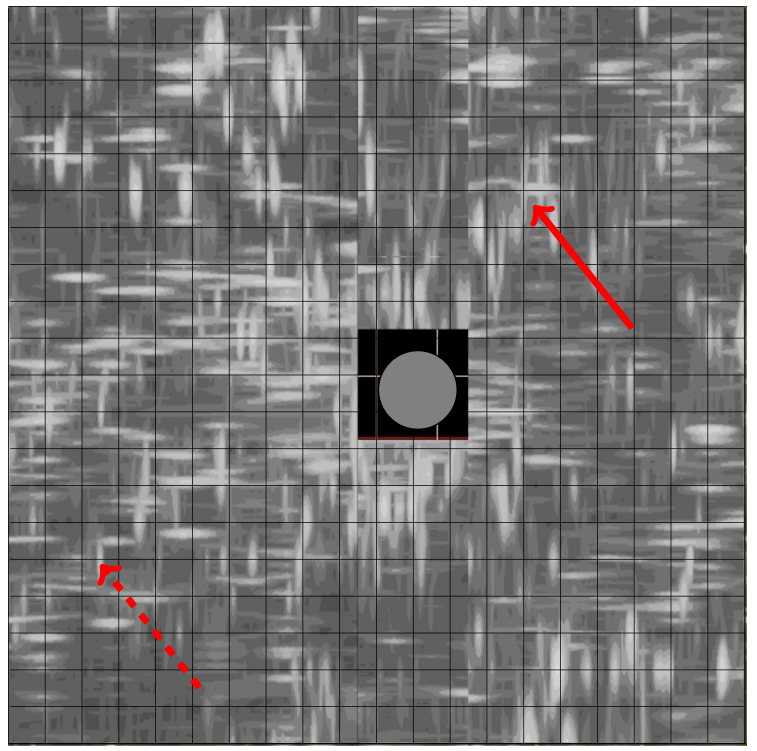

a) $60 \mathrm{~cm}$

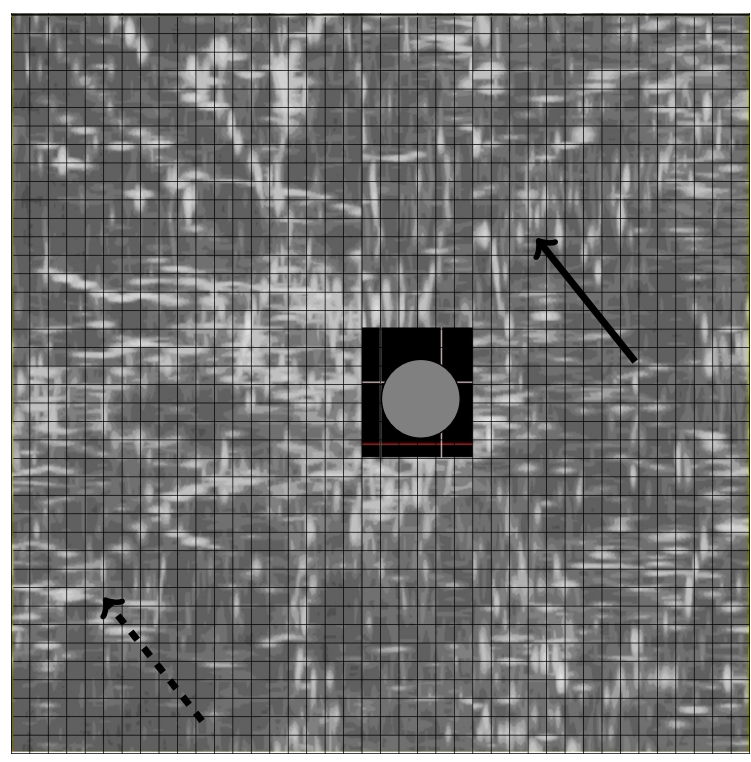

c) $30 \mathrm{~cm}$

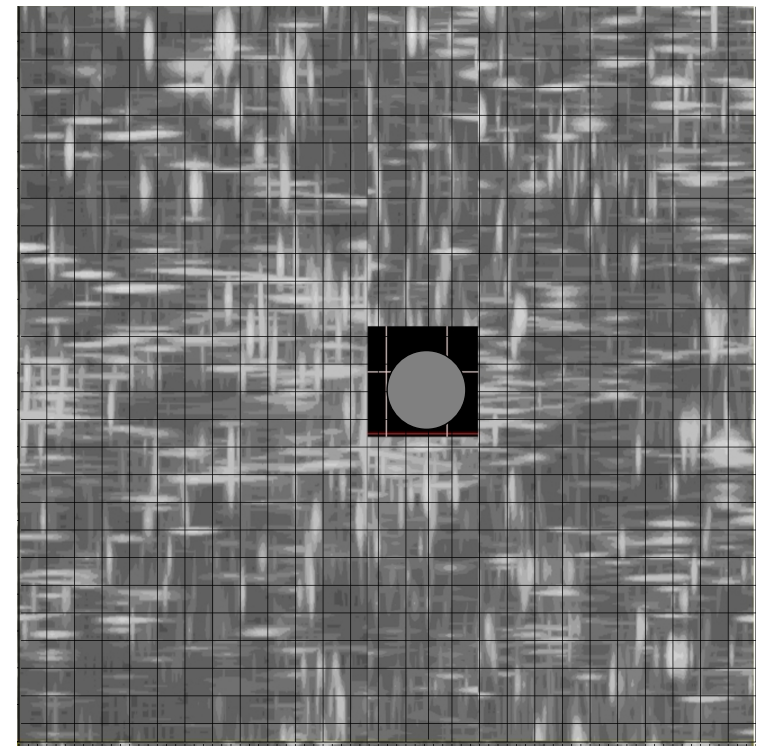

b) $45 \mathrm{~cm}$

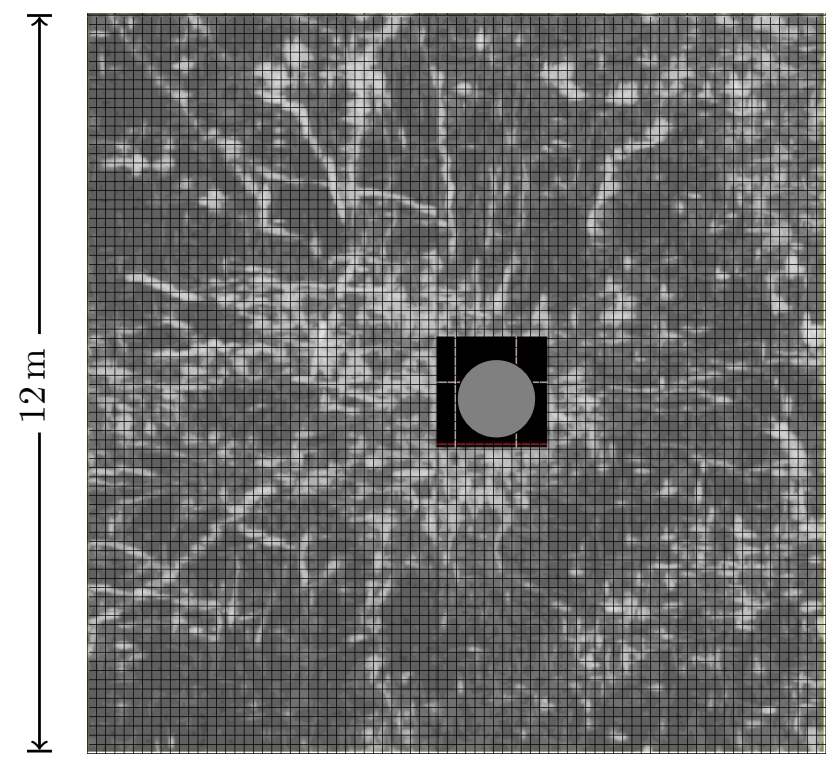

d) $15 \mathrm{~cm}$

Abbildung 4.15: Unterschiedliche Rasterabstände bei 3D-Georadarmessungen (bearbeitete Daten): Darstellung der Messergebnisse einer Georadarmessung an einer Schwarzkiefer auf der Göttinger Schillerwiese. Die Ergebnisdarstellung erfolgt in Form von Horizontalschnitten der unbearbeiteten Rohdaten. Grundlage der Horontalschnitte ist der gleiche Datensatz. Die Abstände der Transekte, auf denen die 3D-Darstellung basiert, werden von $60 \mathrm{~cm}$ in $4.15 \mathrm{a}$ bis $15 \mathrm{~cm}$ in $4.15 \mathrm{~d}$ jeweils um $15 \mathrm{~cm}$ verringert. Der Baum befindet sich jeweils im Bereich der schwarzen Fläche, die nicht befahren werden konnte.

Mit den Parametern z-Wert $=9$; width $=3 ; v=0,07 \mathrm{~m} \mathrm{~ns}^{-1} ; \varepsilon_{r}=18,36$ ergibt sich ein Tiefenbereich von $26,25 \mathrm{~cm}-36,75 \mathrm{~cm}$. 


\subsubsection{Reproduzierbarkeit}

Als Beispiel für die Reproduzierbarkeit von Bodenradarmessungen bei der Wurzeldetektion wurden zwei Vergleichsmessungen im Bereich der Messfläche Schwarzkiefer (Tabelle 3.1 Baum 2a) gewählt. Dazu wurde zusätzlich zu der bereits bei der Analyse der Rasterabstände dargestellten Messung vom 07.04.09 eine weitere Datenaufnahme auf der westlichen Seite der Schwarzkiefer durchgeführt. Diese Seite der Messfläche wurde ausgewählt, da bei der Erstuntersuchung in diesem Bereich die deutlichsten Wurzelverläufe ausgemacht werden konnten. Die zweite Messung wurde ein Jahr später am 14.04.10 vorgenommen. Wie in Abbildung 4.16 zu erkennen ist, verlaufen die Transekte bei der Wiederholungsmessung unter einem Winkel von etwa $2,5^{\circ} \mathrm{zu}$ den bei der ersten Messung aufgenommenen B-Scans. Dies ist dem Umstand geschuldet, dass hier versuchsweise ein Netz zur Aufbringung der Rasterlinien auf der Untersuchungsfläche eingesetzt worden ist, das sich aufgrund seines hohen Eigengewichts nicht exakt wie bei der ersten Datenaufnahme positionieren ließ. Die zweite Befahrung erfolgte, als die Schnüre des Netzes bei minimaler Abweichung (s.o.) vom ersten Raster rechtwinklig zueinander gespannt waren. Um eine Vergleichbarkeit zwischen den Messergebnissen zu ermöglichen, wurden für die Darstellung des unteren Horizontalschnitts ein z-Wert von 5 ns und eine Zeitscheibenbreite (width) von 5 ns verwendet; die Einstellungen beim oberen Horizontalschnitt betragen 6 ns für den z-Wert und 3 ns für die Zeitscheibenbreite. Diese Einstellungen wurden unter der Maßgabe vorgenommen, trotz möglicherweise anderer $\varepsilon_{\mathrm{r}}$-Werte aufgrund verschiedener Bodenfeuchten bei den Messungen eine Kongruenz zwischen den Ergebnisdarstellungen in Abbildung 4.16 herzustellen.

In den beiden Zeitscheiben lassen sich zahlreiche Übereinstimmungen finden. Neben den prägnanten Starkwurzeln, die im oberen und unteren Bild vorhanden sind, stimmen auch die Bereiche, in denen keine starken Reflexionen vorkommen, überein. An Stellen, an denen nur in einem Bild über längere Distanzen Reflexionen verzeichnet werden, findet sich im anderen Bild ein schwaches unterbrochenes Lineament.

Abbildung 4.17 zeigt die Ergebnisse einer mit dem Programm ImageJ und dem plug-in Image CorrelationJ (Chinga und Syverud 2007) durchgeführten Kreuzkorrelation, um die Ergebnisse der beiden Messungen statistisch auf mögliche Übereinstimmungen testen zu können. Die unterschiedlichen Winkel bei der Datenaufnahme $\left(2,5^{\circ}\right)$ wurden bei der Korrelation berücksichtigt.

Bei der Bildkorrelation, bei der jeder einzelne Pixel von Abbildung 4.17a mit dem entsprechenden Pixel von 4.17b abgeglichen worden ist, ergibt sich ein Korrelationskoeffizient von $R=0,47$. Die korrespondierenden Pixel der beiden Bilder sind in Abbildung 4.17c zu sehen. Die Bildkorrelation, die auf dem Durchschnittswert von Quadraten mit einer Kantenlänge von 15 Pixeln basiert, geht mit einem Korrelationskoeffizienten von $\mathrm{R}=0.70$ einher. Die zugehörige Bildüberlagerung befindet sich in Abbildung 4.17d, der dazugehörige scatterplot in Abbildung 4.17e.

Ein weiteres, nicht so hochauflösendes Beispiel für die Reproduzierbarkeit von Bodenradarmessungen bei der Wurzeldetektion befindet sich im Anhang (Abbildung A.12). 


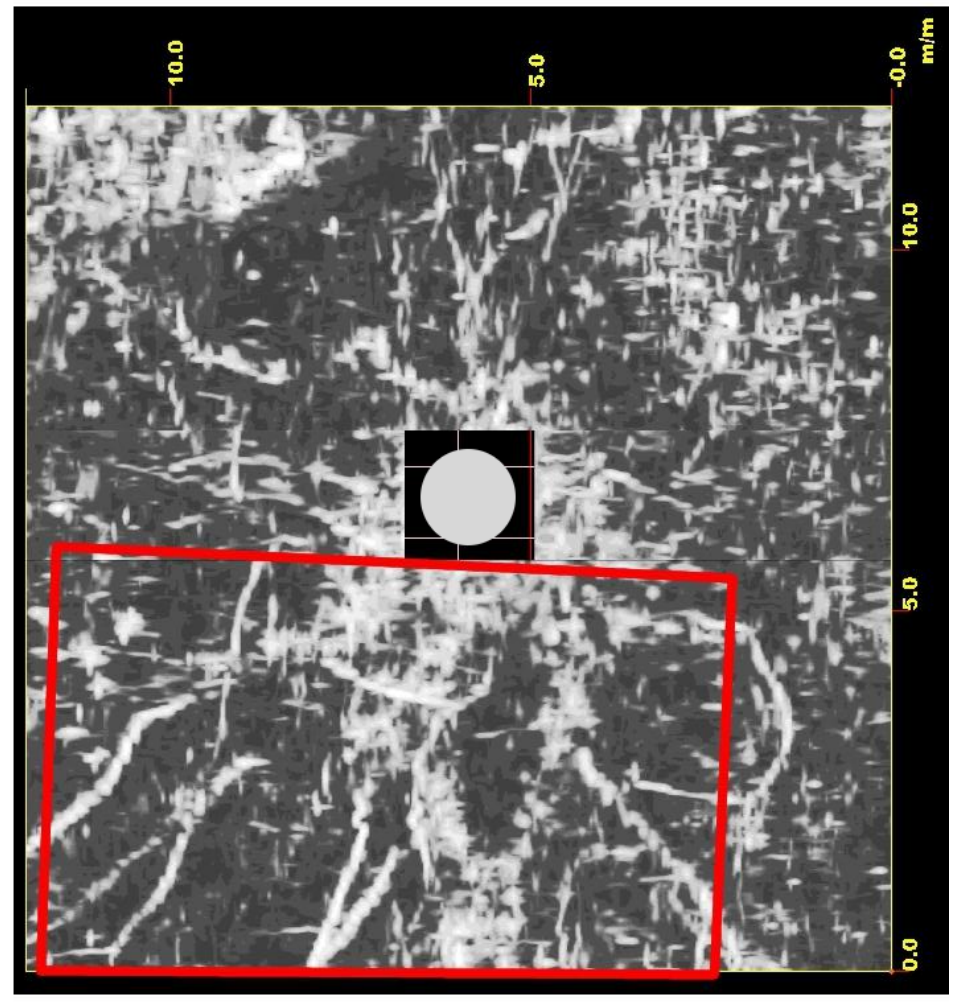

a) Messung an der Schwarzkiefer am 17.04.09. Die Parameter $\mathrm{z}$-Wert $=6$; width $=3 ; v=0,066 \mathrm{~m} \mathrm{~ns}^{-1} ; \varepsilon_{r}=20,66$ ergeben einen Tiefenbereich von $14,85 \mathrm{~cm}-24,75 \mathrm{~cm}$.

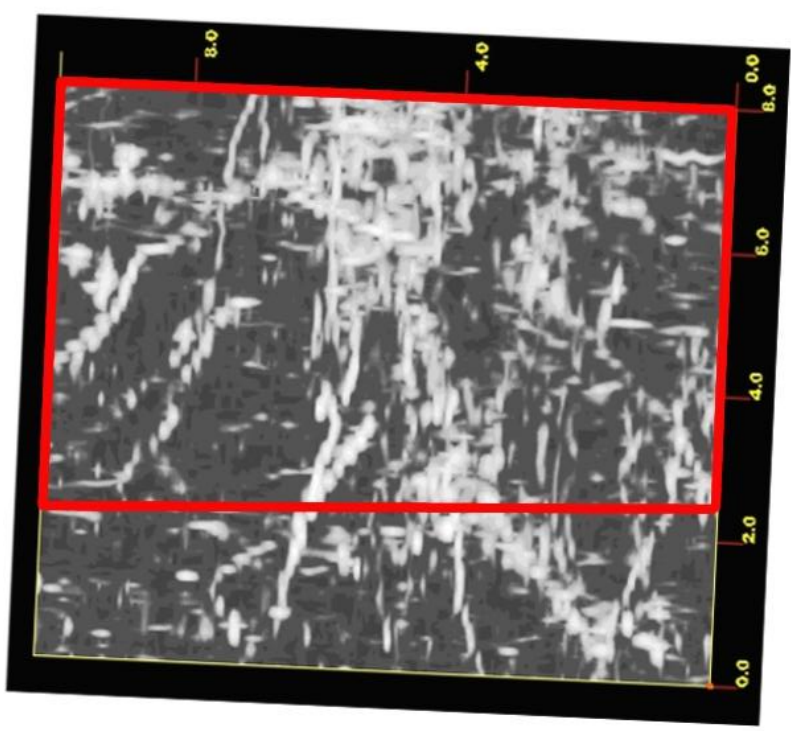

b) Messung an der Schwarzkiefer am 14.04.10. Die Parameter $\mathrm{z}$-Wert $=5 ;$ width $=5 ; v=0,067 \mathrm{~m} \mathrm{~ns}^{-1} ; \varepsilon_{r}=20,05$ ergeben einen Tiefenbereich von $8,36 \mathrm{~cm}-25,13 \mathrm{~cm}$.

Abbildung 4.16: Reproduzierbarkeitsmessung an der Schwarzkiefer auf der Göttinger Schillerwiese. 


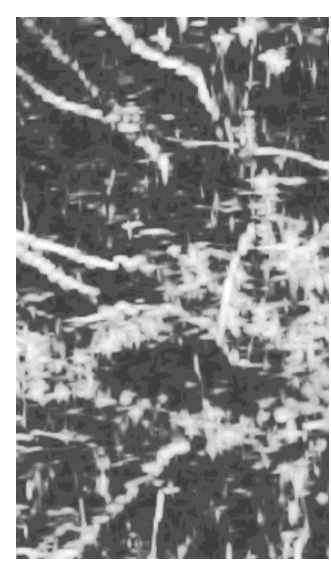

a) Messung 1

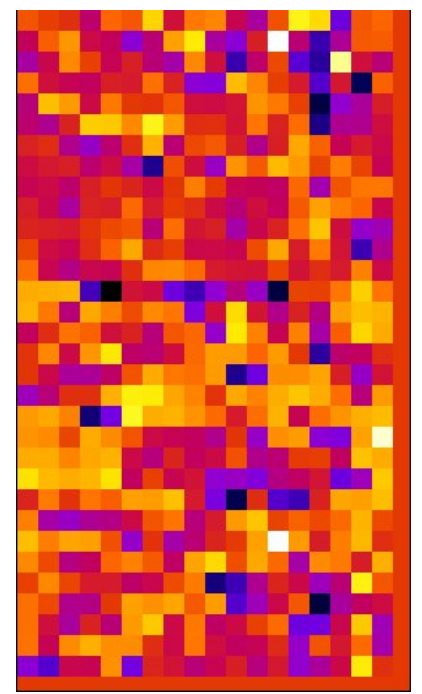

d) correlation-map bei 15Pixel-Vergleich

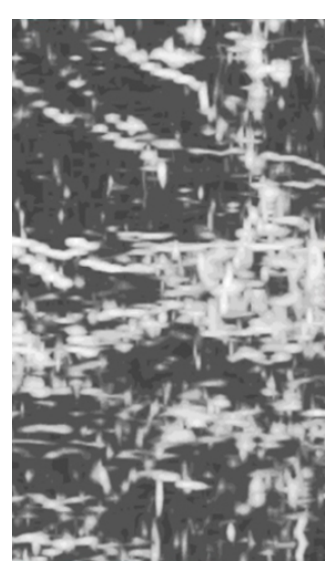

b) Messung 2

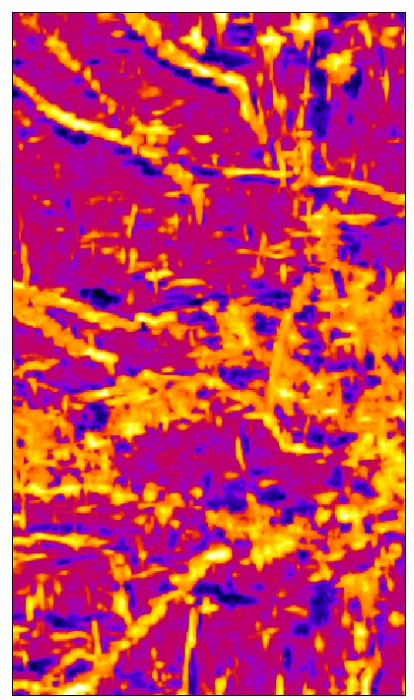

c) correlation-map bei 1 Pixel-Vergleich

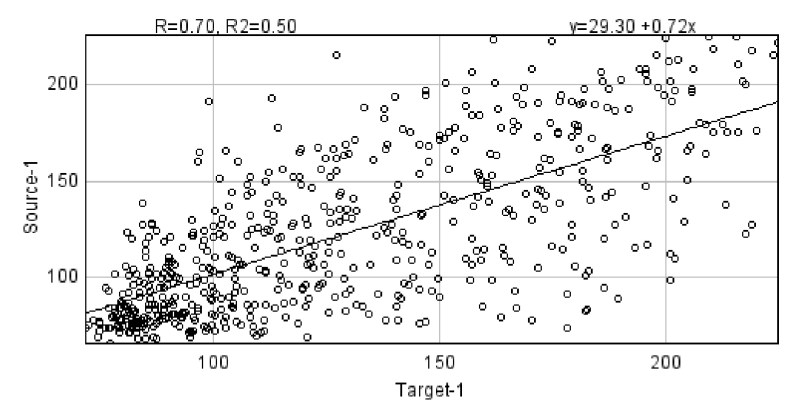

e) scatterplot des 15-Pixel-Vergleichs

Abbildung 4.17: Untersuchung der Reproduzierbarkeit durch Bildkorrelation. 


\subsubsection{Nachbearbeitung und Filter}

Von Abbildung 4.18a bis Abbildung 4.18h wird am Beispiel der Schwarzkiefer auf der Göttinger Schillerwiese (Tabelle 3.1, Baum 2a) gezeigt, wie sich verschiedene Filtertechniken und Bearbeitungsschritte in RADAN 6.6 (vgl. Kapitel 2.3.2.3) auf die Visualisierung von Baumwurzeln in den Messergebnissen von Georadaruntersuchungen auswirken. Dazu sind in der linken Bildhälfte Horizontalschnitte, bei denen bestimmte Filter eingesetzt worden sind, dargestellt. In der rechten Bildhälfte befinden sich 2D-Radargramme, die auf dem Transekt $y=9.0 \mathrm{~m}$ aufgenommen worden sind. Dabei sind außer bei Abbildung 4.18c und Abbildung 4.18d immer Horizontalschnitte und 2D-Radargramme, die mit dem gleichen Filter bearbeitet worden sind, in einer Zeile gegenübergestellt. Bei Merge Regions lässt sich der Effekt des Bearbeitungsschritts nicht in der 2D-Darstellung erkennen und bei dem Bearbeitungsschritt Zero Positioning nicht in der gewählten 3D-Darstellung. Daher wurde in beiden Fällen auf die Gegenüberstellung der 2Dbeziehungsweise 3D-Darstellung verzichtet.

Die Ergebnisse lassen sich wie folgt beschreiben:

- Durch den Bearbeitungsschritt Merge Regions werden die einzelnen Teilmessflächen zu einer großen Messfläche in der Ergebnisdarstellung zusammengefasst; die in 4.18a vorhandenen Vertikalschnitte in den einzelnen Teilfächen werden entfernt. Außerdem wird der bei der Datenaufnahme ausgesparte Bereich, in dem der Baum steht, mit einer Graustufe belegt (Abbildung 4.18d).

- Durch die Anwendung von Zero Positioning werden die systeminternen Signale, die vor der Bodenreflexion auftreten, entfernt (Abbildung 4.18d).

- Der eingesetzte Background Removal-Filter hat keine großen Auswirkungen auf die Ergebnisdarstellung in der Form eines Horizontalschnitt (Abbildung 4.18e). In der 2D-Darstellung ist deutlich erkennbar, dass lineare Strukturen mit einem horizontalen Verlauf deutlich reduziert werden, wodurch Hyperbeln und andere Anomalien, die auf Objekte im Untergrund hinweisen, deutlicher hervortreten (Abbildung 4.18f).

- Die Migration führt dazu, dass die einzelnen Wurzelstränge im Horizontalschnitt (Abbildung 4.18g schmaler als in den vorangestellten Abbildungen dargestellt werden. In der 2D-Darstellung ist erkennbar, dass die Äste der einzelnen Hyperbeln (Abbildung 4.18h) eliminiert wurden. 


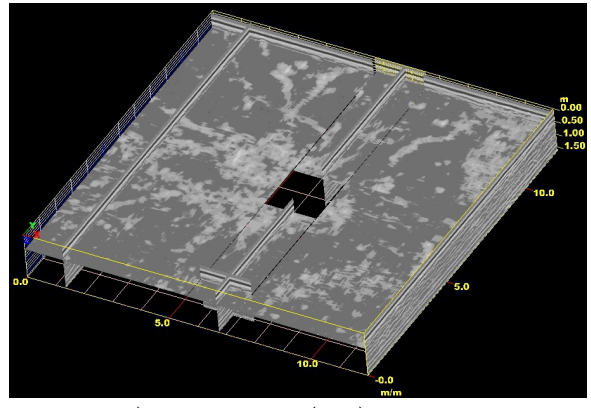

a) Rohdaten (3D)

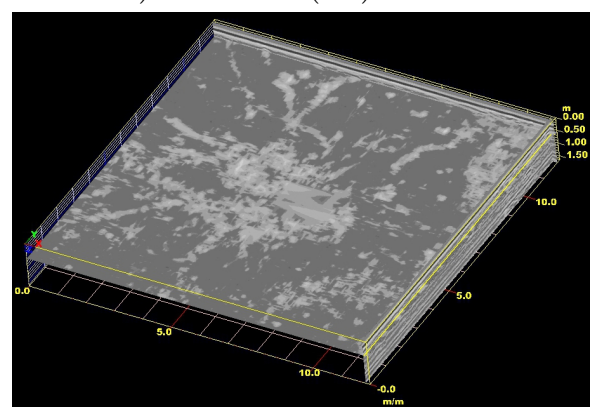

c) Merge Regions (3D)

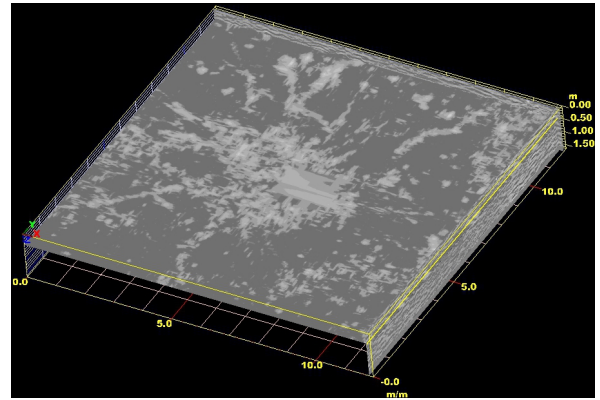

e) Background Removal (3D)

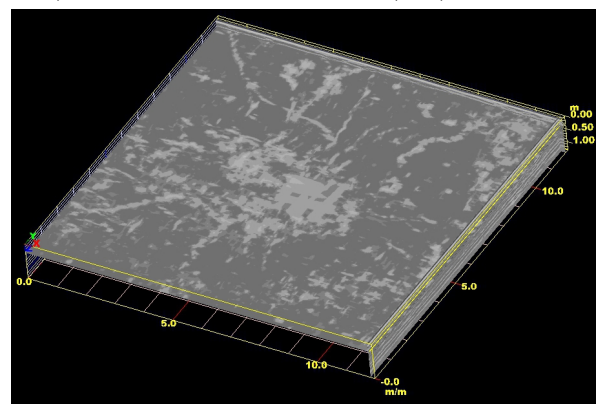

g) Migration (3D)

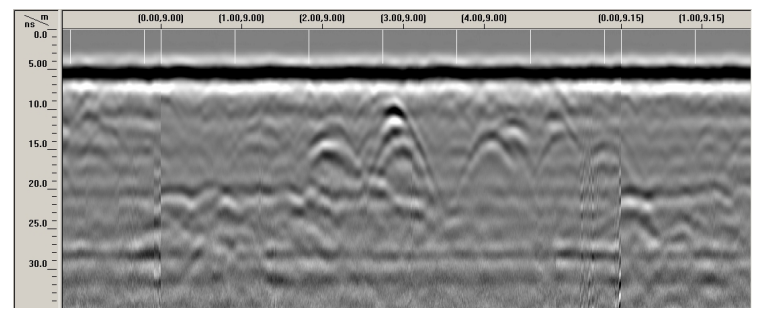

b) Rohdaten (2D)

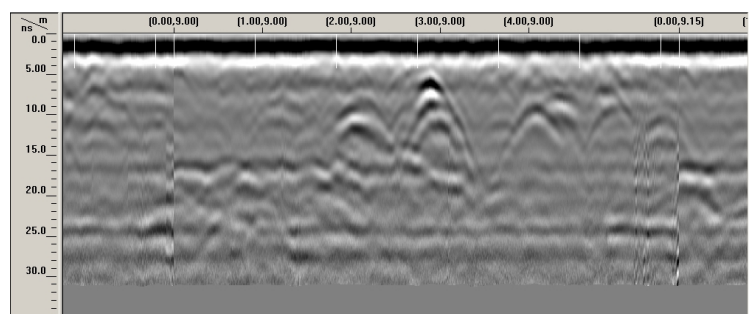

d) Zero Positioning (2D)

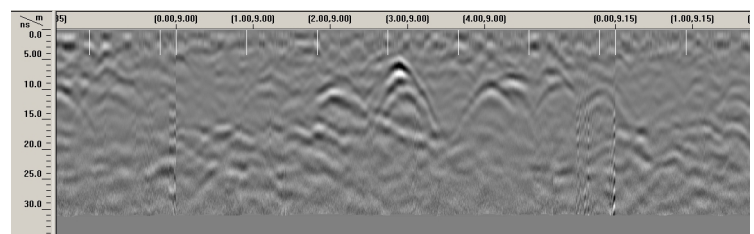

f) Background Removal (2D)

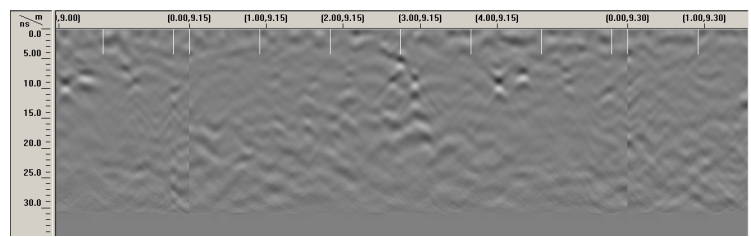

h) Migration (2D)

Abbildung 4.18: Bearbeitung von Bodenradardatensätzen mit unterschiedlichen Filtern. 


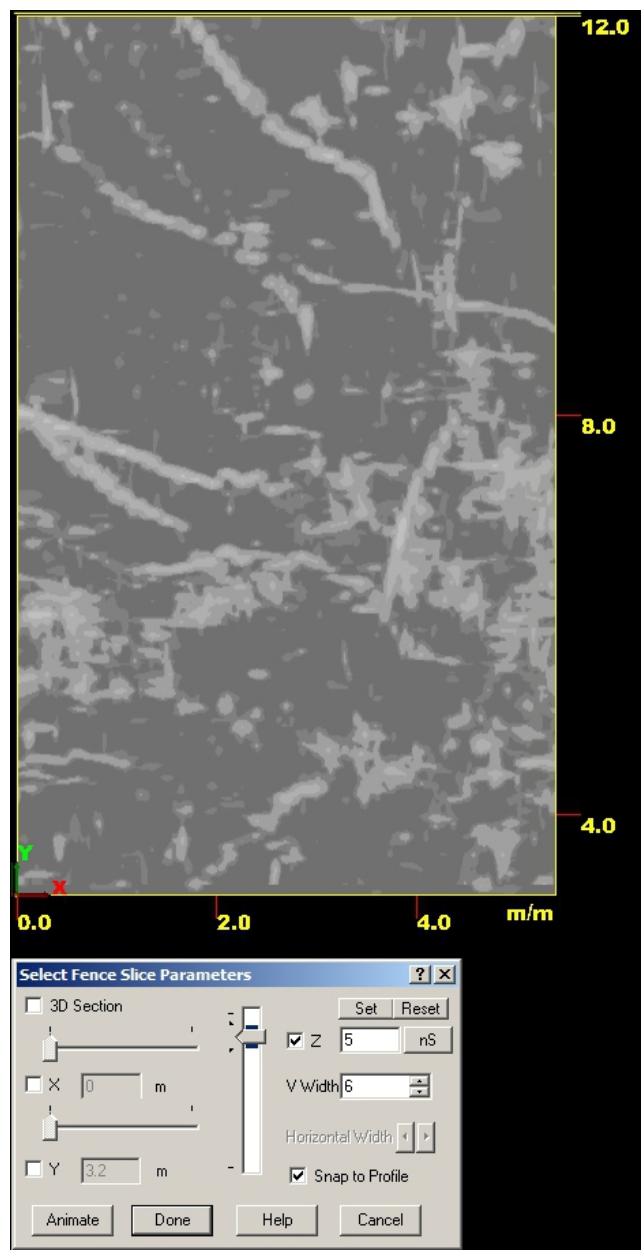

a) $400 \mathrm{MHz}$

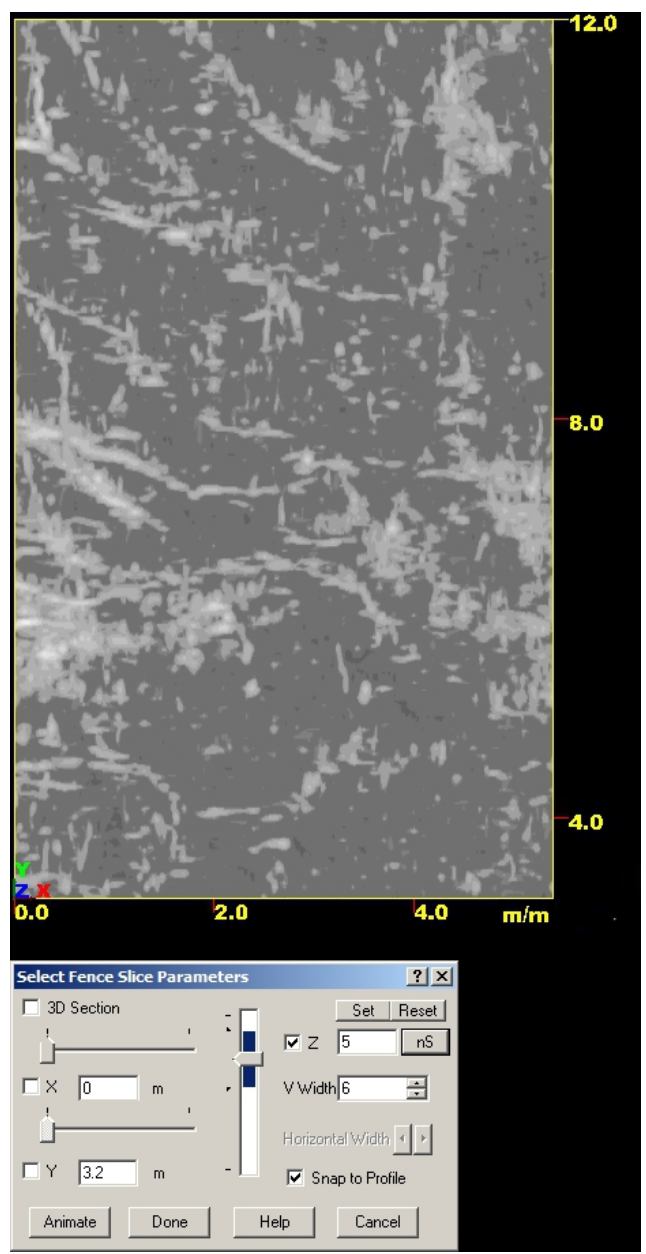

b) $900 \mathrm{MHz}$

Abbildung 4.19: Einfluss verschiedener Messfrequenzen auf das Messergebnis an der Schwarzkiefer.

Mit den Parametern z-Wert $=5$; width $=6 ; v=0,07 \mathrm{~m} \mathrm{~ns}^{-1} ; \varepsilon_{r}=18,36$ ergibt sich ein Tiefenbereich von $7,0 \mathrm{~cm}-28,0 \mathrm{~cm}$ für beide Horizontalschnitte.

\subsubsection{Messfrequenz}

Es ist bekannt, dass sich die Mittenfrequenz der eingesetzten Messantenne auf die Auflösung bei Bodenradarmessungen auswirkt. Abbildung 4.19 zeigt einen Vergleich von zwei Horizontalschnitten, die auf der Messfläche Schwarzkiefer (Tabelle 3.1 Baum 2a) mit zwei unterschiedlichen Antennen aufgezeichnet worden sind. Eingesetzt wurden die $400 \mathrm{MHz}-$ Antenne (Abbildung 4.19a), die auch für die Aufnahme der übrigen in dieser Arbeit präsentierten Radardaten verwendet worden ist, und eine geliehene $900 \mathrm{MHz}$-Antenne (Abbildung 4.19b.

Beim Vergleich der beiden Horizontalschnitte lassen sich in beiden Abbildungen die wesentlichen Wurzelstrukturen wiedererkennen. Bei der mit der $900 \mathrm{MHz}$-Daten erstellten Aufnahme treten dabei noch weitere Wurzeln hinzu, die im anderen Datensatz nicht zu erkennen sind. Strukturen, die in beiden Abbildungen auftreten, werden im $900 \mathrm{MHz}$-Horizontalschnitt bis auf einen Fall, in dem ein flächiges Muster vorliegt, feiner aufgelöst. Partiell sind die Objekte, die durch die höhere Auflösung erst deutlich hervortreten, schon im $400 \mathrm{MHz}-$ Horizontalschnitt mit einem etwas schwächeren Kontrast zum Hintergrund zu erkennen. 


\subsubsection{Verifikation der Ergebnisse}

\subsubsection{Großflächige Wurzelfreilegungen}

Einsteinufer Landwehrkanal Am Einsteinufer zeigen sich deutliche Übereinstimmungen zwischen den im Radargramm identifizierten Wurzelverläufen und den tatsächlichen Gegebenheiten im Untergrund (Abbildung 4.20 und 4.21.

Beim Silberahorn konnten die anhand des Radarergebnisses getätigten Befunde in etwa 10,0 m und 12,0 m Entfernung vom Baum bestätigt werden. Die Durchmesser der freigelegten Wurzeln liegen zwischen 4,0 cm (Grobwurzel nach FLL 2006) und 8,0 cm (Starkwurzel); die Tiefen der Wurzeloberkanten liegen zwischen $6,0 \mathrm{~cm}$ und $20,0 \mathrm{~cm}$.

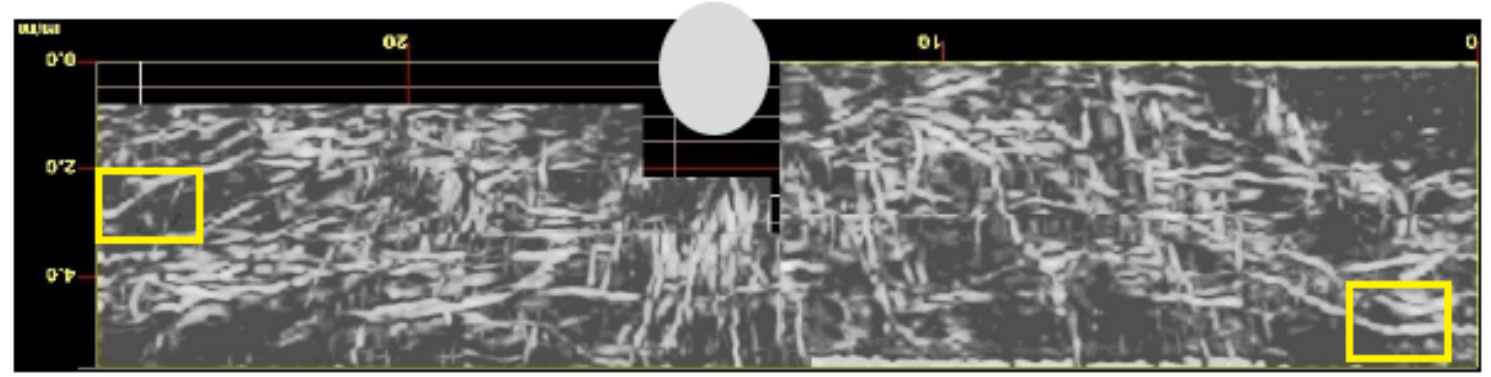

a) Radargramm der Messfäche mit Lage der freigelegten Bereiche. Die Parameter z-Wert = 2; width = 3; $v=0,1 \mathrm{~m} \mathrm{~ns}^{-1} ; \varepsilon_{r}=9$ ergeben einen Tiefenbereich von $2,5 \mathrm{~cm}-17,5 \mathrm{~cm}$.
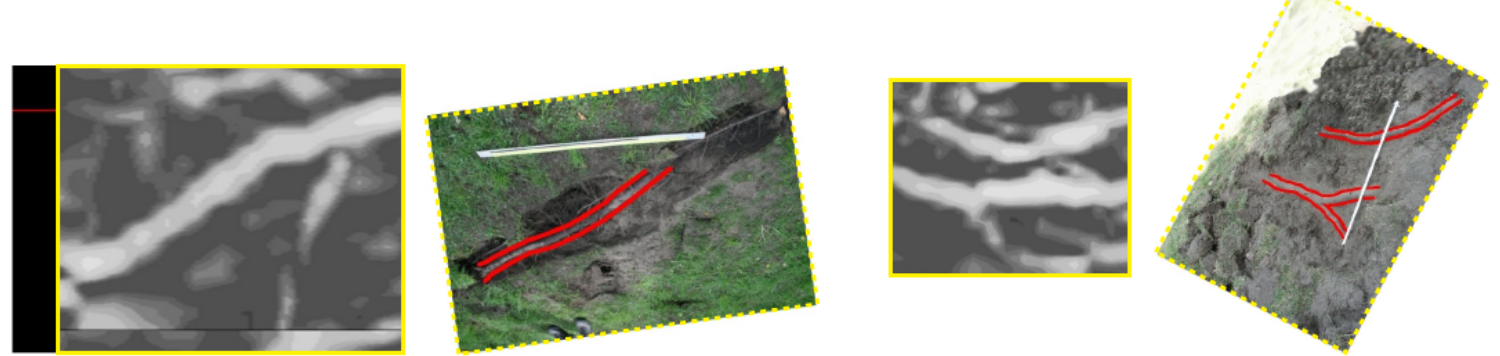

b) Vergrößerter Ausschnitt des Radargramms (jeweils links) und freigelgte Wurzel (jeweils rechts).
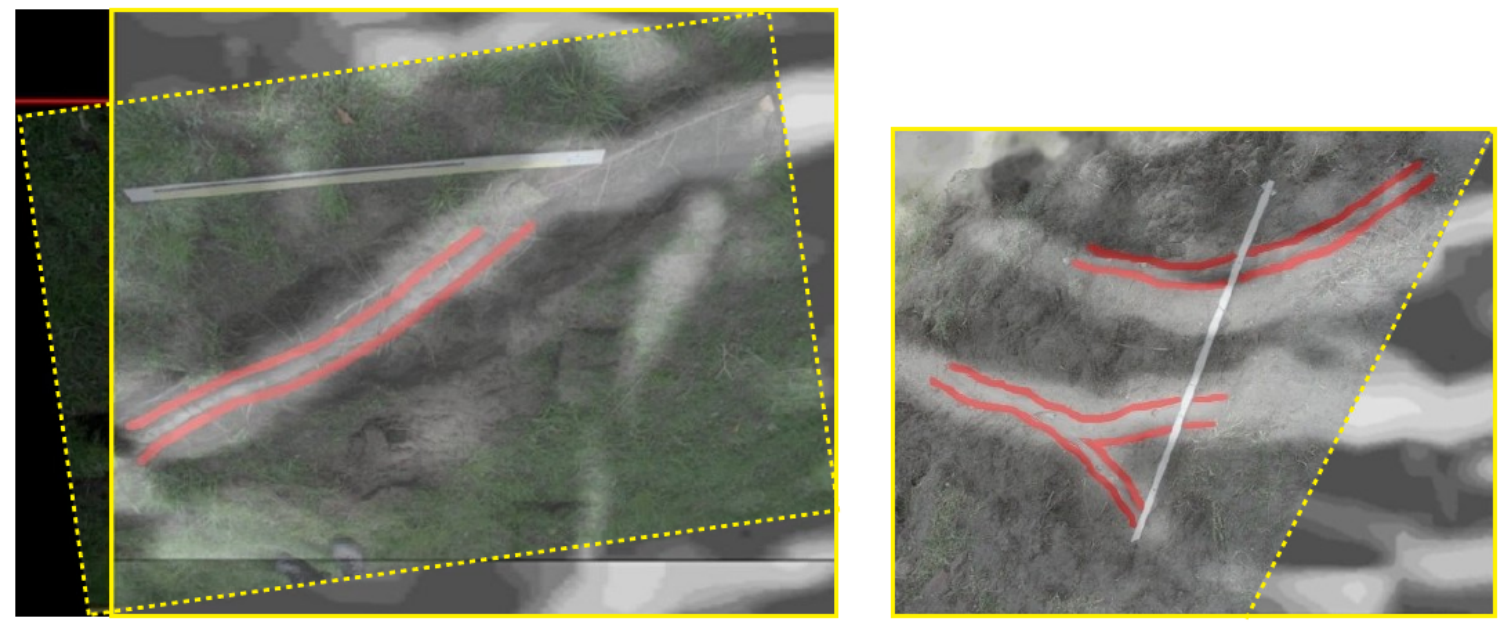

c) Überlagerung von Radargramm und Wurzelfreilegung.

Abbildung 4.20: Wurzelfreilegung auf der Messfläche Silberahorn Landwehrkanal. 
Bis auf eine Freilegung auf der südöstlichen Seite der Hybidpappel in unmittelbarer Baumnähe, bei der Steine freigelegt wurden, konnten auch auf der zweiten Messfläche am Einsteinufer bei allen Freilegungen Wurzeln an den Positionen der im Radargramm identifizierten Verdachtsstellen gefunden werden. Besonders auffällig ist hier die gute Übereinstimmung zwischen freigelegter Wurzel und zugeordneter Anomalie im rechten Bildteil von Abbildung 4.21, bei der die Anomalie ohne offensichtliche Verbindung zum Baum vom Bereich der Ufermauer in die Messfläche läuft. Die Durchmesser der Wurzeln liegen bei dieser Messfläche zwischen 3,1 cm (Grobwurzel) und 4,5 cm (Grobwurzel); die Tiefen der Wurzeloberkanten liegen zwischen 2,0 cm und 4,0 cm.

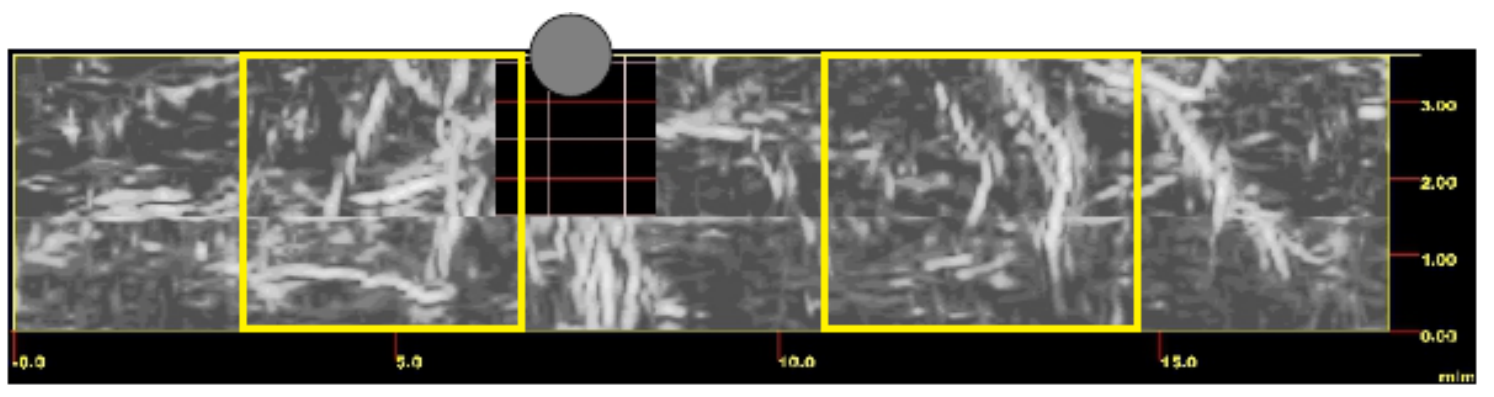

a) Radargramm der Messfläche mit Lage der freigelegten Bereiche. Die Parameter z-Wert = 4; width = 4; $v=0,1 \mathrm{~m} \mathrm{~ns}^{-1} ; \varepsilon_{r}=9$ ergeben einen Tiefenbereich von $12,5 \mathrm{~cm}-27,5 \mathrm{~cm}$.
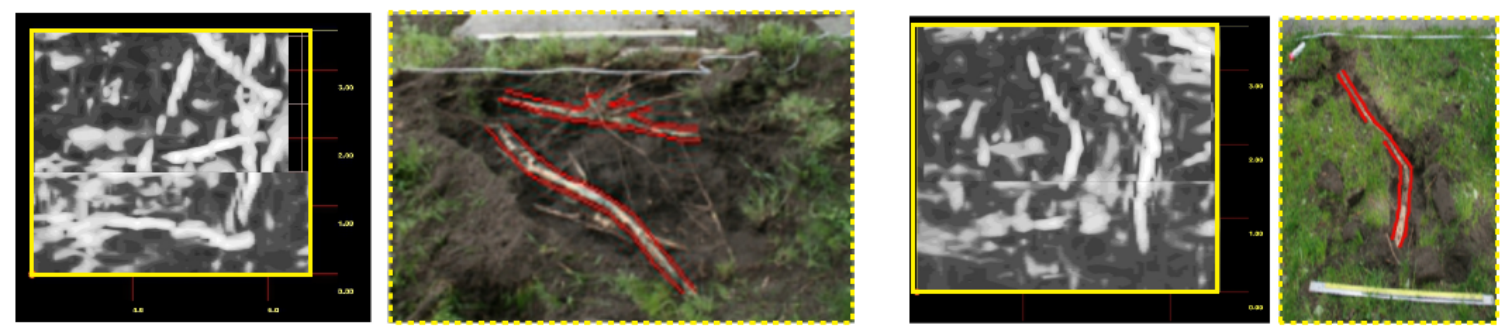

b) Freigestellter Ausschnitt des Radargramms (jeweils links) und freigelegte Wurzel (jeweils rechts).
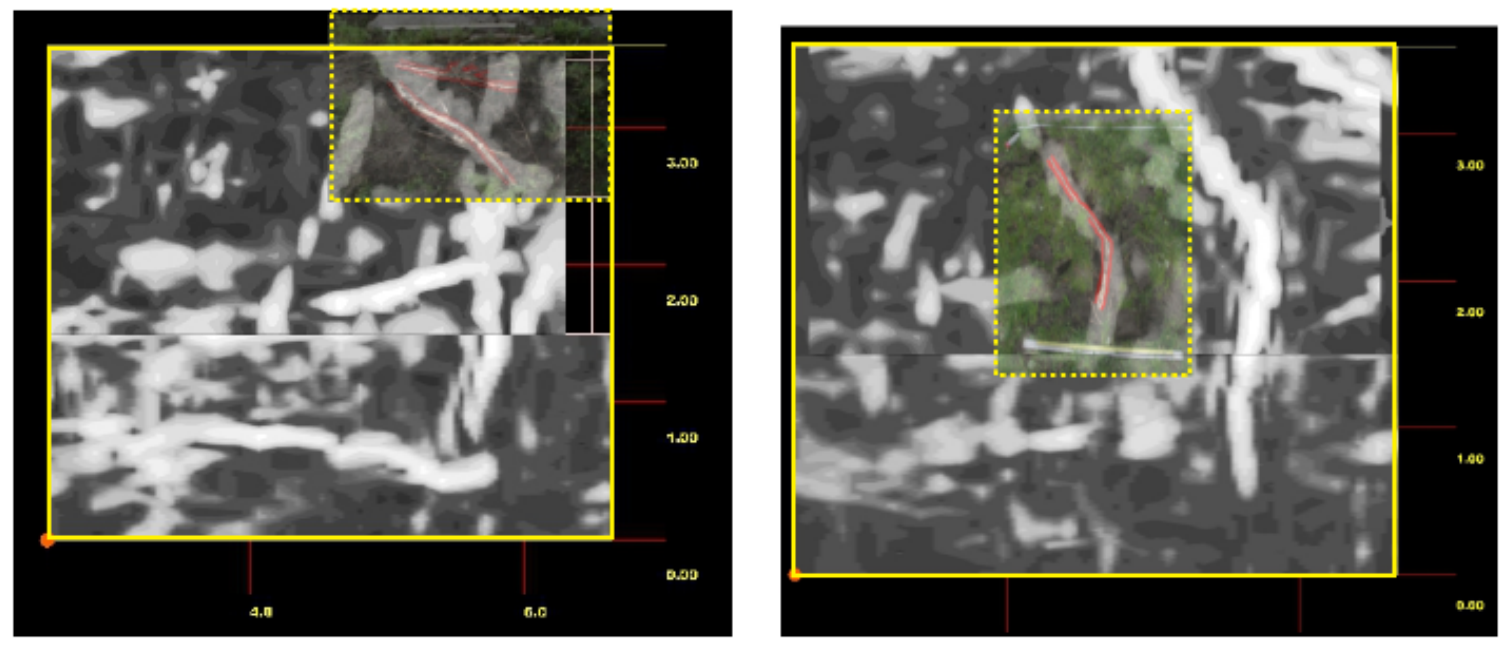

c) Überlagerung von Radargramm und Wurzelfreilegung.

Abbildung 4.21: Wurzelfreilegung auf der Messfläche Hybridpappel Landwehrkanal. 
Kastanie Kreuzkirche Fulda Auch auf der Messfläche in Fulda konnte die aus dem Ergebnis der Radarmessung abgeleitete Aussage über die Wurzelverhältnisse durch die Freilegungen bestätigt werden (Abbildung 4.22).

Zusätzlich konnte bei dieser Freilegung auch der Negativbefund bezüglich der Durchwurzelung der linken Hälfte der Messfläche bestätigt werden, da der entsprechende Teil des zur Verifikation angelegten Wurzelsuchgrabens wurzelfrei ist. Die lokal begrenzten Reflexionen im linken Teil des Radargramms wurden wahrscheinlich durch den höheren Tongehalt in diesem Teil der Untersuchungsfläche hervorgerufen. Die freigelegten Wurzeln liegen in Tiefen von $12,0 \mathrm{~cm}$ bis $15,0 \mathrm{~cm}$ (Wurzeloberkanten) und weisen Durchmesser zwischen 6,0 cm und 7,0 cm (Starkwurzeln) auf. 


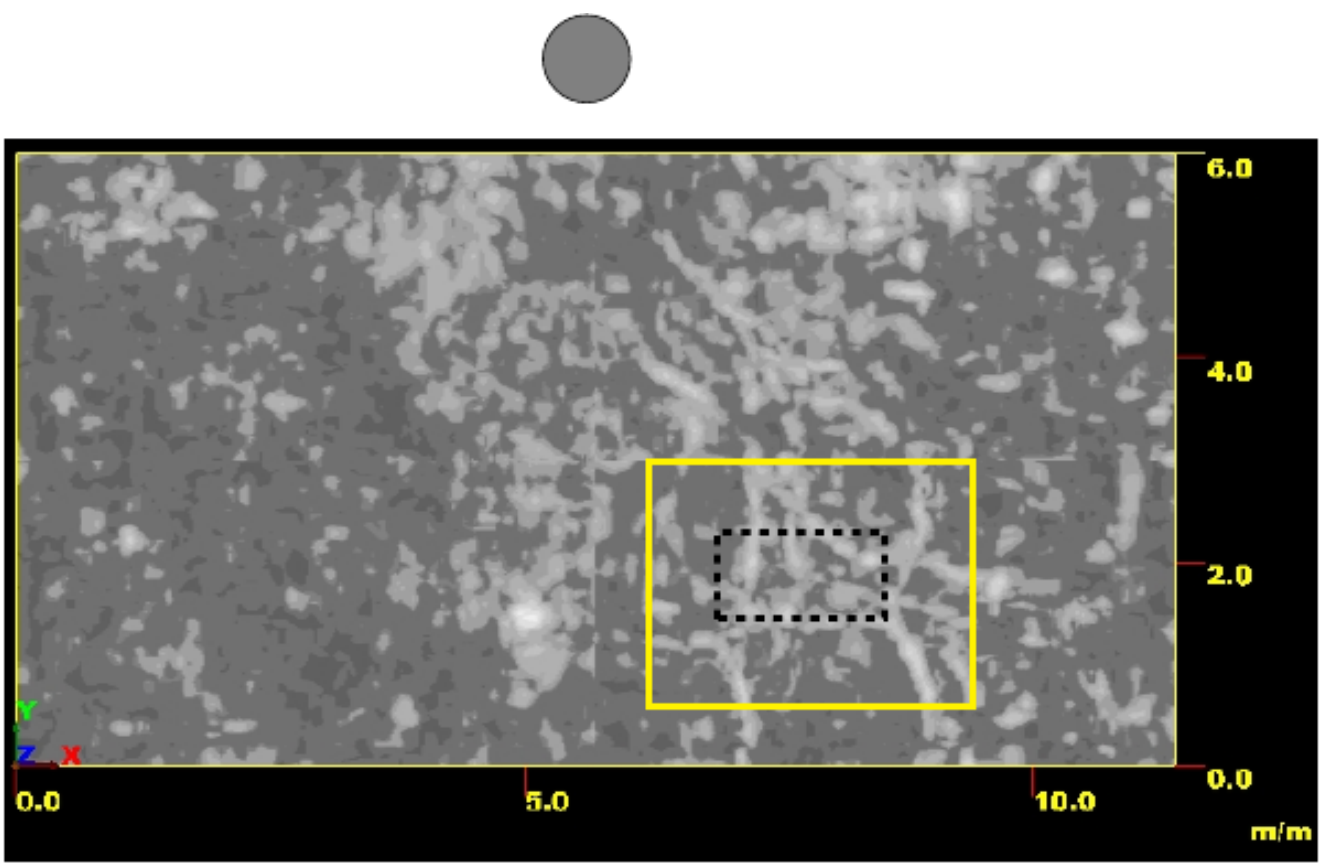

a) Radargramm der Messfläche mit Lage der freigelegten Bereiche. Die Parameter z-Wert $=8$; width $=3 ; v=0,06 \mathrm{mns}^{-1} ; \varepsilon_{r}=25$ ergeben einen Tiefenbereich von $19,5 \mathrm{~cm}-28,5 \mathrm{~cm}$.
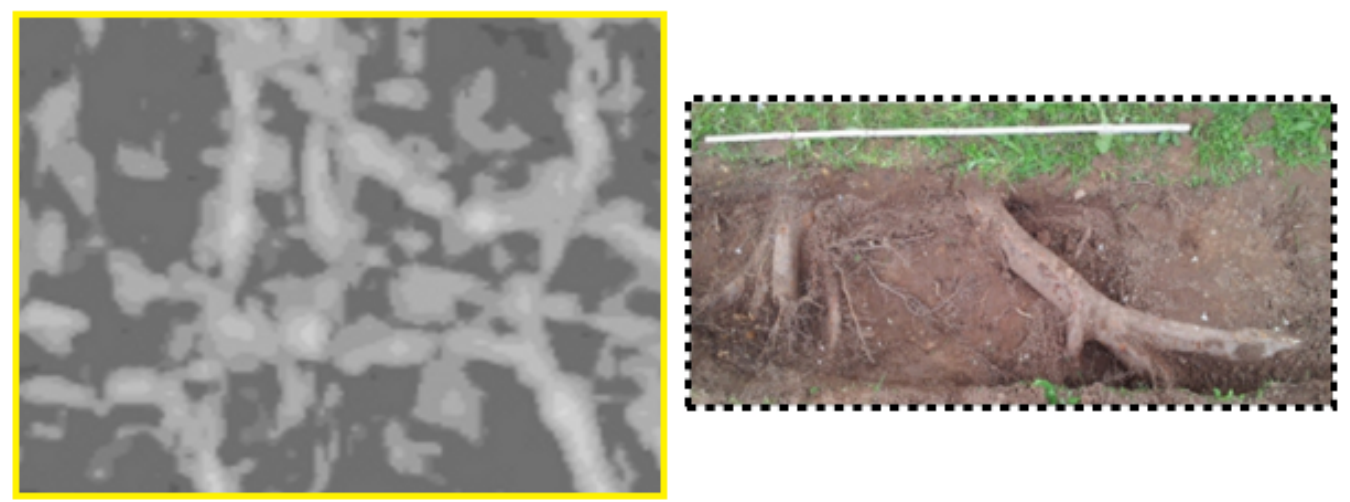

b) Vergrößerter Ausschnitt des Radargramms (links) und freigelgte Wurzel (rechts).

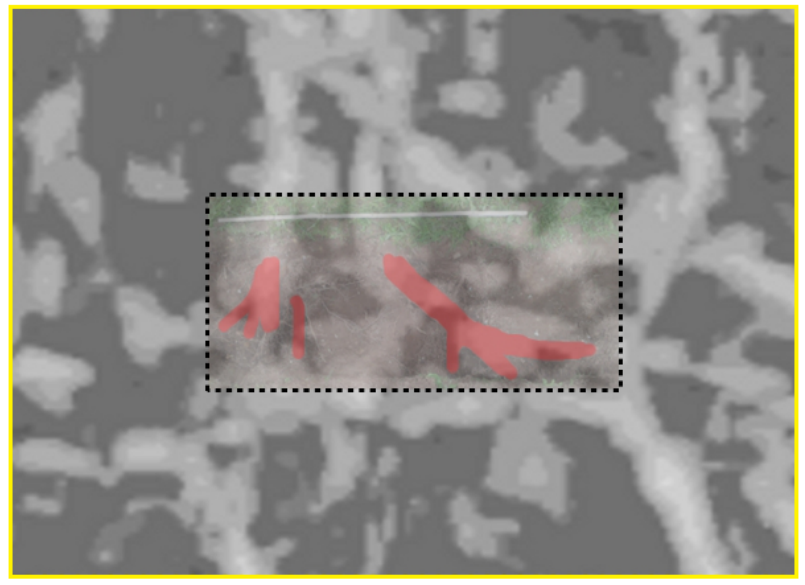

c) Überlagerung von Radargramm und Wurzel (rot markiert).

Abbildung 4.22: Wurzelfreilegung auf der Messfläche Kastanie Kreuzkirche Fulda. 
Corneliusufer Landwehrkanal Im Gegensatz zu den Flächen am Einsteinufer und in Fulda liegen in den Radargrammen der Messflächen Kleine und Große Kastanie am Corneliusufer keine deutlichen Wurzelindikationen vor. Daher wurden Bereiche freigelegt, in denen das Radarergebnis eventuell Wurzeln ausweisen könnte und Bereiche, in denen aufgrund der Nähe zum Baum gröbere Wurzeln zu erwarten sind (Abbildung 4.23).

Abbildung 4.23 zeigt zwei Aufgrabungen auf der Messfläche Große Kastanie. In einem Fall wurde eine Starkwurzel mit einem Durchmesser von $6,5 \mathrm{~cm}$ in einer Tiefe von $10,0 \mathrm{~cm}$ freigelegt, deren Lage mit der Position einer lokal begrenzten Anomalie im Radargramm übereinstimmt. Bei der anderen Aufgrabung auf der Messfläche konnte an der Position einer Anomalie, die eventuell als Wurzel interpretiert werden kann, keine Wurzel bei der Aufgrabung identifiziert werden. Stattdessen wurde ein Untergrundaufbau vorgefunden, der durch einen hohen Anteil gröberer Pflastersteinfragmente in einer stark verdichteten, sandigen Matrix gekennzeichnet ist. Dieser Aufbau konnte bei einer weiteren Grabung in der Mitte der Messfläche bis in eine Tiefe von $70,0 \mathrm{~cm}$ bestätigt werden.

Die Aufgrabungsergebnisse auf der benachbarten Messfläche Kleine Kastanie zeigen ebenfalls eine hohe Konzentration von Pflastersteinen im Untergrund, beinhalten aber ebenso wenig Grobwurzeln. In Baumnähe zeigt sich eine hohe Wurzeldichte, die die Größenklassen Feinwurzeln und Schwachwurzeln bis $1,5 \mathrm{~cm}$ umfasst.

Da weder durch die Radarmessungen noch durch die Wurzelgrabungen weitergehende Erkenntnisse über den Aufbau der Wurzelsysteme an diesem Standort erbracht werden, wird nicht nur die Grenze der Wurzeldetektion anhand von Bodenradarmessungen, sondern auch insgesamt die Grenze der Verifikation der Ausprägung von Wurzelsystemen auf den Messflächen am Corneliusufer erreicht. Anzunehmen ist, dass der an diesem Standort vorgefundene komplexe Untergrundaufbau (Bodenbeschaffenheit und Morphologie des Wurzelsystems) zu einem Reflexionsmuster führt, anhand dessen sich das Wurzelwerk nicht detektieren lässt. 
4.2 3D-Georadarmessungen zu grundlegenden Fragestellungen

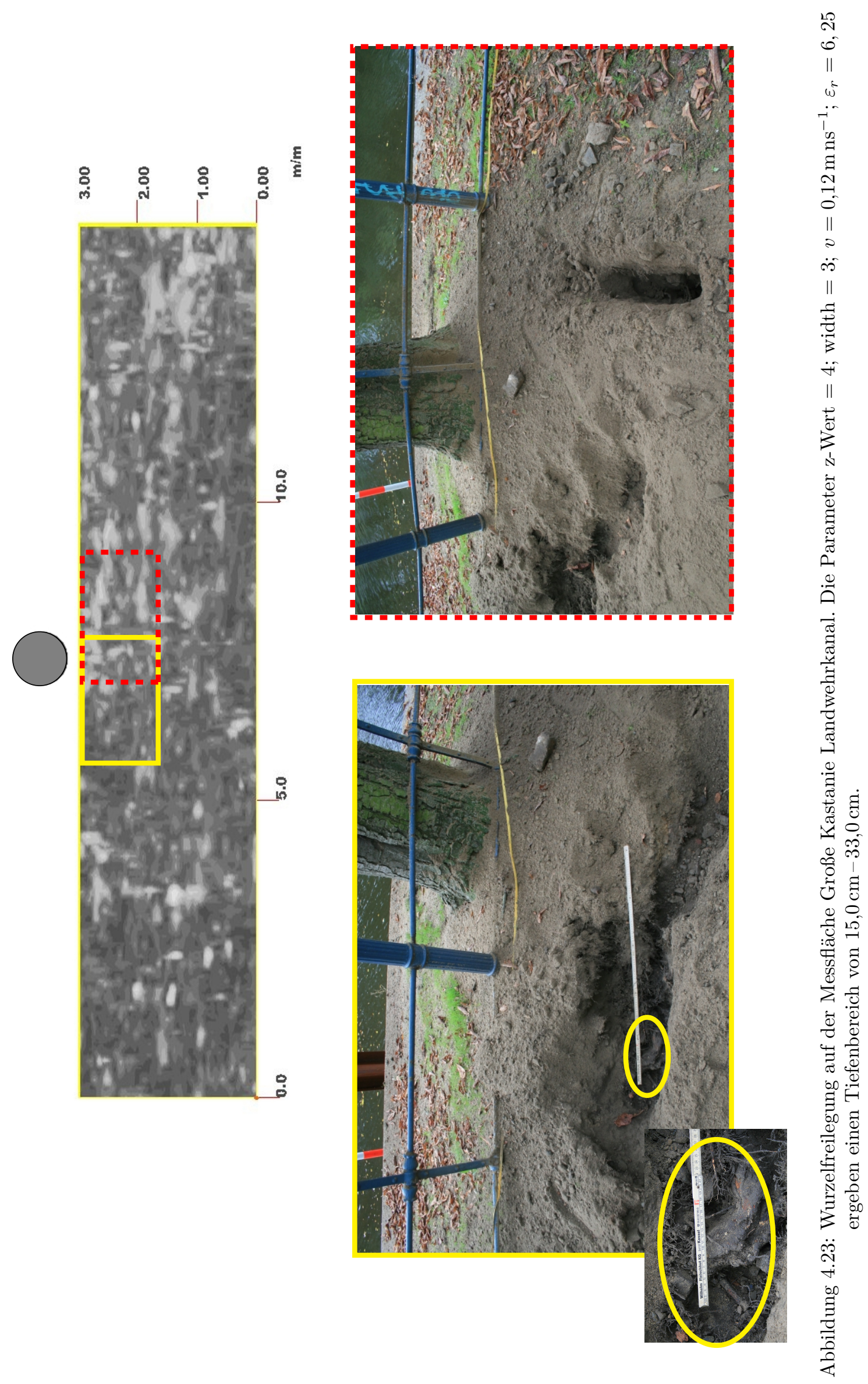



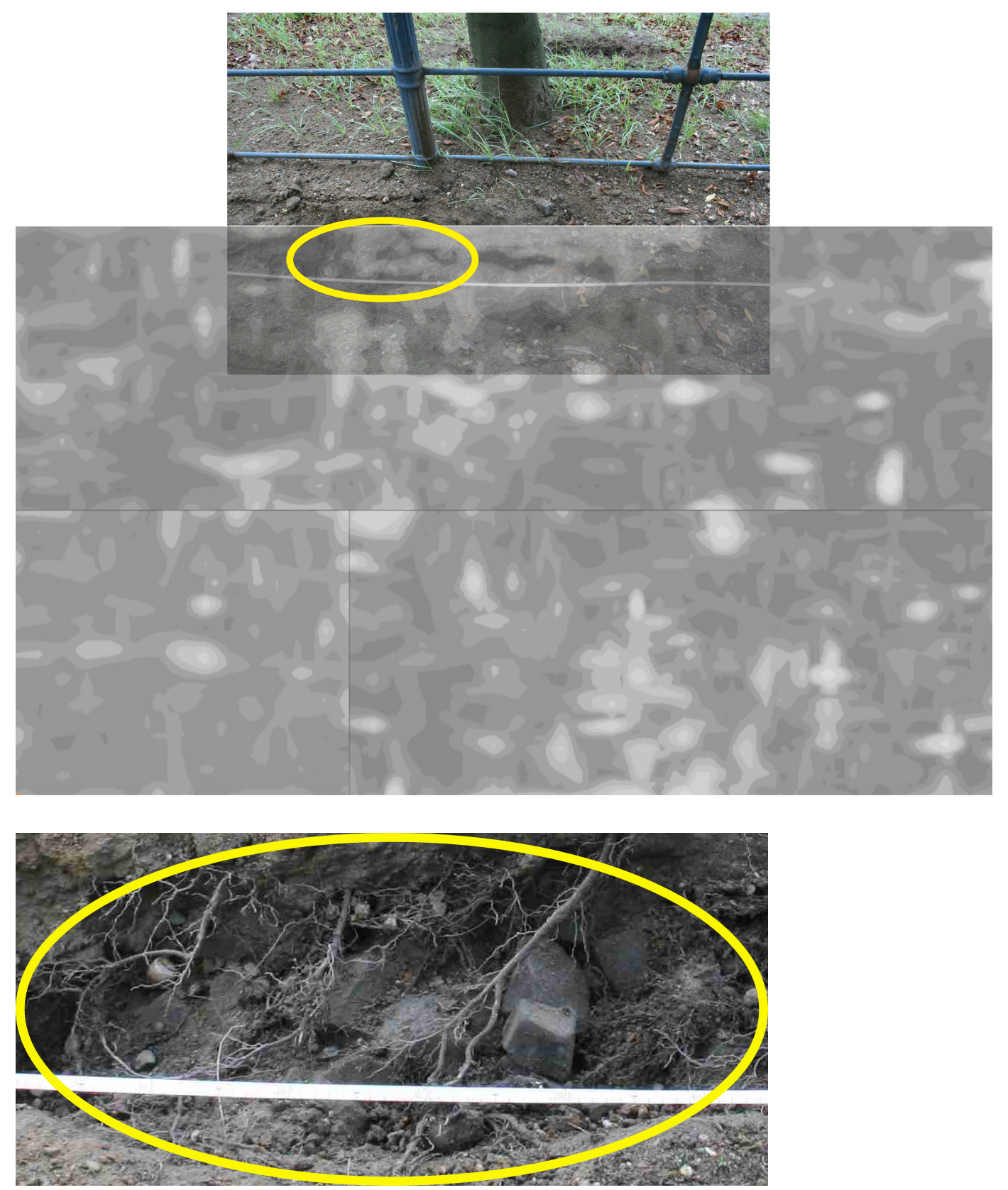

Abbildung 4.24: Wurzelfreilegung auf der Messfläche Kleine Kastanie Landwehrkanal. Die Parameter $\mathrm{z}$-Wert $=3$; width $=5 ; v=0,12 \mathrm{~m} \mathrm{~ns}^{-1} ; \varepsilon_{r}=6,25$ ergeben einen Tiefenbereich von $3,0 \mathrm{~cm}-33,0 \mathrm{~cm}$. 
Kastanie Archipelstraat Nimwegen Die begrenzten Möglichkeiten der Rekonstruktion von Wurzelsystemen aus Bodenradarmessungen und ihrer Verifikation werden auch anhand von Airspade-Freilegungen an einer in Nimwegen untersuchten Kastanie deutlich (Abbildung 4.25).

Die Grenzen der GPR-Wurzeldetektion werden daran ersichtlich, dass im Ergebnisradargramm nur in wenigen Bereichen Strukturen identifiziert werden können, die darauf hindeuten, dass hier Teile des Wurzelsystems der Kastanie zu erkennen sind.

Der Einsatz des Airspades unter den entfernten Gehwegplatten zeigt ausschließlich feinere Wurzeln (Schwachwurzeln bis 2,0 cm nach FLL 2006), die keine Deckungsgleichheit mit den Strukturen in den Radargrammen aufweisen. Den Ergebnissen der Messungen in den Versuchsbecken zufolge, können Wurzeln dieses Durchmessers mit dem eingesetzten Messequipment nicht detektiert werden.

Wie am Corneliusufer in Berlin sind auch hier die Grenzen des eingesetzten GPR-Equipments zur Wurzeldetektion erreicht. Darüber hinaus ist es auch bei dieser Messfläche nicht möglich, mit dem Aispade Wurzeln in größerer Tiefe in dem stark verdichteten Boden freizulegen. 

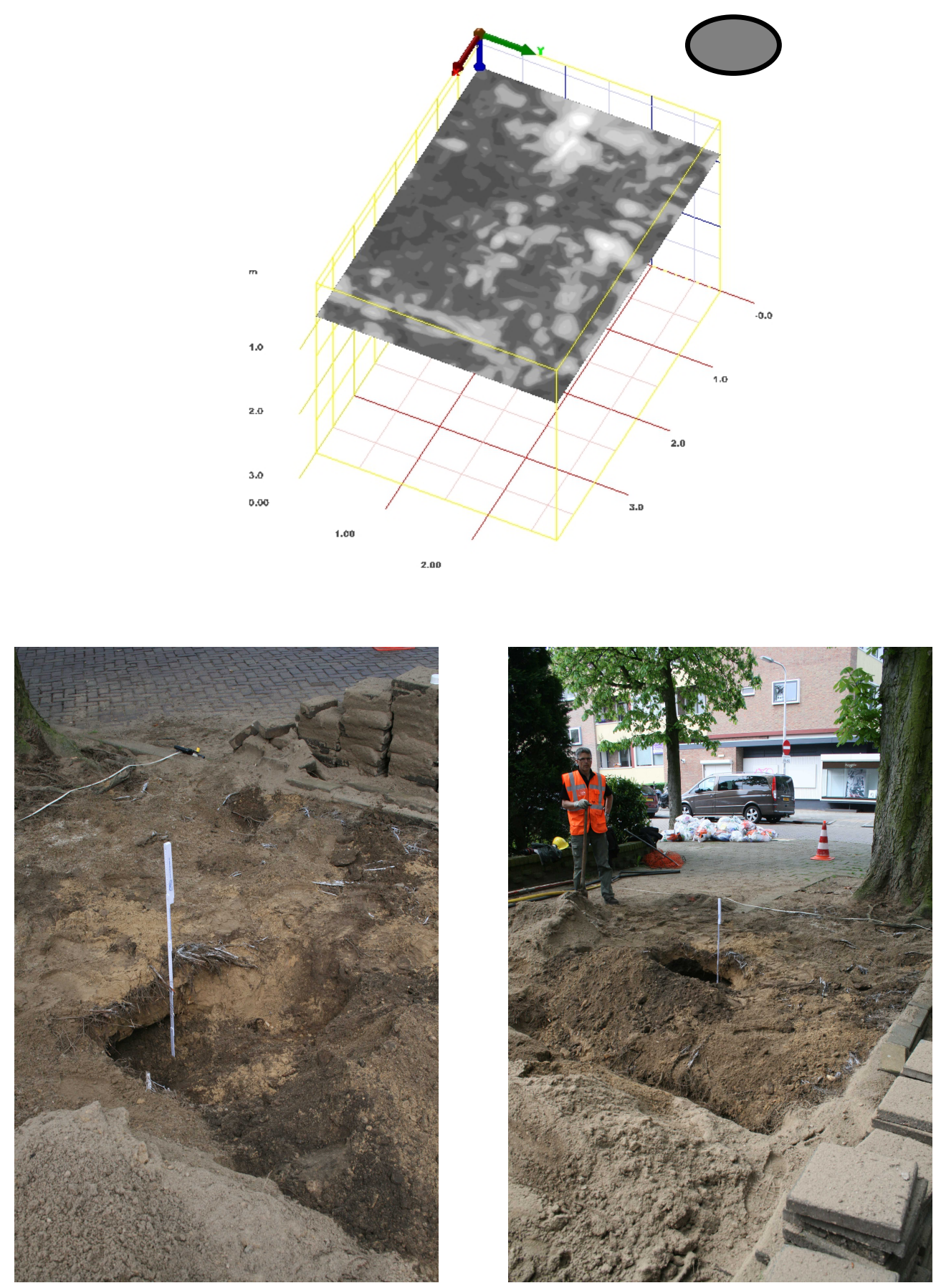

Abbildung 4.25: Wurzelfreilegung auf der Messfläche Kastanie Archipelstraat Nimwegen. Mit den Parametern z-Wert $=10$; width $=4 ; v=0,12 \mathrm{mns}^{-1} ; \varepsilon_{r}=6,25$ ergibt sich ein Tiefenbereich von $48,0 \mathrm{~cm}-72,0 \mathrm{~cm}$. 


\subsubsection{Ergebnisse der kleinräumigen Freilegungen}

Die Ergebnisse der Radarmessungen im Bereich der Fichte und Fichtengruppe auf der Göttinger Schillerwiese konnten durch kleinräumige Aufgrabungen bestätigt werden. Bei $100 \%$ der Beprobungen ( $\mathrm{in}$ Abbildung 4.26a und 4.26b markiert die freigelegten Bereiche im Radargramm) konnten Wurzeln gefunden werden.

Die Durchmesser der freigelegten Wurzeln bei der Fichtengruppe liegen zwischen 1,4 cm und 20,5 cm; die Tiefe der Wurzeln zwischen 1,0 cm und 19,0 cm. Bei der solitären Fichte wurden Wurzeln mit Durchmessern zwischen $1,9 \mathrm{~cm}$ und $16,5 \mathrm{~cm}$ in Tiefen zwischen $0,0 \mathrm{~cm}$ und $17,0 \mathrm{~cm}$ an den Probepunkten freigelegt. Auffällig war die deutliche Rotfäule, die an den Wurzeln der beiden Messflächen vorherrscht. Diese hat den Messergebnissen zufolge das Gelingen der Wurzeldetektion aber nicht negativ beeinflusst.

Als Grundlage für die Präsentation der Messungen im folgenden Kapitel lässt sich festhalten, dass Strukturen, die aufgrund ihrer Lage und ihres Verlaufs auf Baumwurzeln hindeuten, in den meisten Fällen auch als solche identifiziert werden konnten, wobei Form und Lage bei den Freilegungen auch mit Form und Lage in den Radargrammen übereinstimmen.

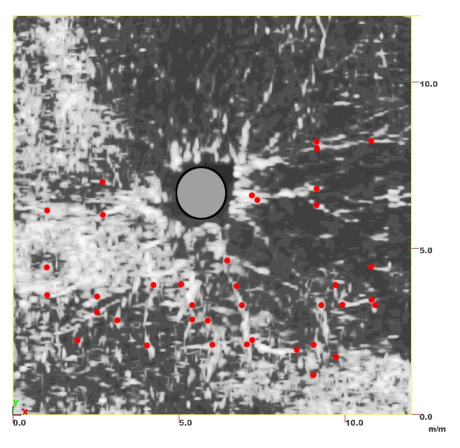

a) Fichte

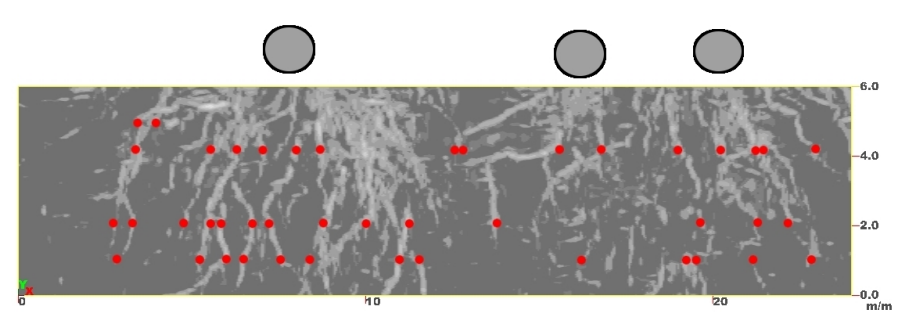

b) Fichtengruppe

Abbildung 4.26: Kleinräumige Freilegungen auf der Göttinger Schillerwiese. 


\subsubsection{Korrelation von Wurzelmasse und Bodenfeuchte mit den auf der Schillerwiese ermittelten Widerstandswerten}

Die Abbildungen 4.27 bis 4.29 zeigen den Zusammenhang zwischen den spezifischen Widerständen und der Wurzelmasse bzw. der Bodenfeuchte an den Beprobungspunkten auf den Messflächen Schwarzkiefer und Buchengruppe. Im oberen Teil der beiden ersten Abbildungen ist dabei jeweils das Ergebnis der Korrelation zu sehen (Abbildung 4.27a und 4.28b), im unteren Teil ist die räumliche Verteilung der Residuen dargestellt, die sich bei den angenommenen Modellen ergeben (Abbildung 4.27b und 4.28b). Die Verteilung der Residuen berücksichtigt dabei gleichzeitig die Wurzelmasse und die Bodenfeuchte.

Der Zusammenhang zwischen Widerstand und Wurzelmasse ist bei der Schwarzkiefer nicht eindeutig. So ist bei drei der beprobten Transekte ein negativer und bei Transekt 3,5 $\mathrm{m}$ ein positiver Verlauf zu erkennen (Abbildung 4.27a). Bei dieser Messfläche zeigen die Residuen ein räumliches Muster, das daran erkennbar ist, dass die negativen und positiven Abweichungen vom Modell jeweils in abgeschlossenen Arealen vorliegen (Abbildung 4.27b).

Bei der Buchengruppe verhält es sich anders. Bei der Wurzelmasse zeigen hier drei Transekte einen deutlichen positiven Verlauf und ein Transekt einen negativen Verlauf (Abbildung 4.28a). Die im Vergleich zur Schwarzkiefer kleineren Beträge der Residuen zeigen, dass das gewählte Modell die Verteilung der Messwerte bei diesem Messbeispiel besser beschreibt (Abbildung 4.28b).

Zwischen spezifischem Widerstand und Bodenfeuchte zeigt sich bei der Schwarzkiefer in drei Fällen ein deutlicher Zusammenhang (Abbildung 4.29a), wobei auch hier sowohl positive als auch negative Zusammenhänge vorliegen. Bei der Buchengruppe besteht bei zwei Transekten ein deutlicher negativer Zusammenhang zwischen Widerstand und Bodenfeuchte (Abbildung 4.29b). 


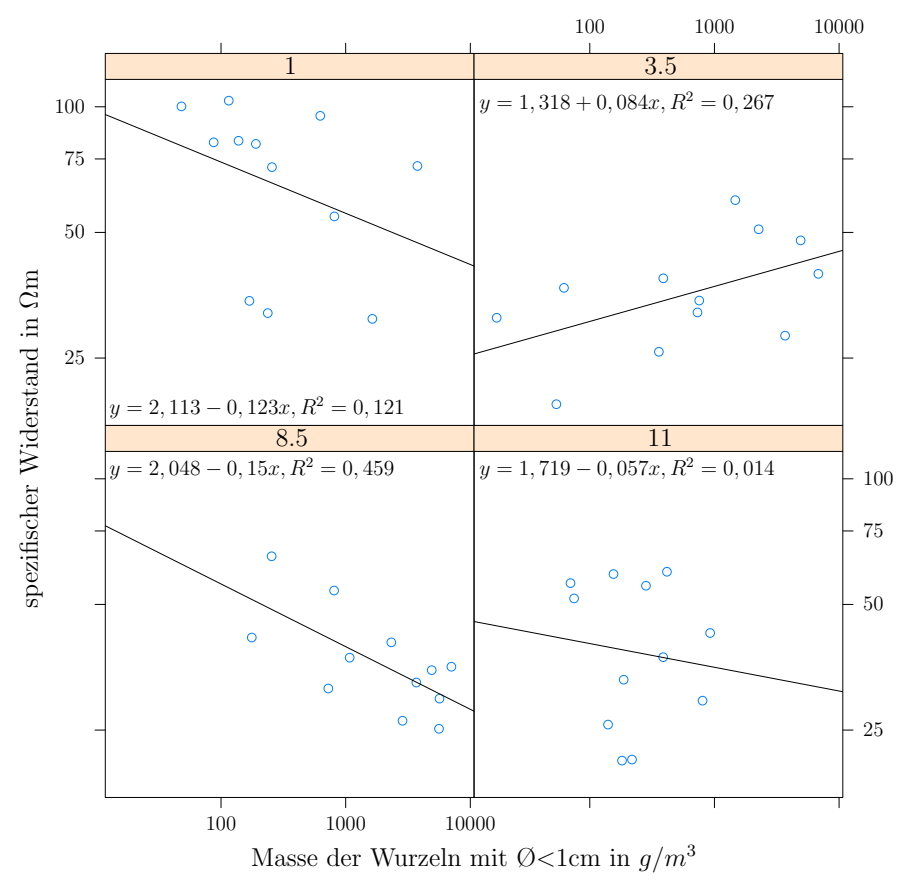

a) Zusammenhang zwischen spezifischem Widerstand und Wurzelmasse.

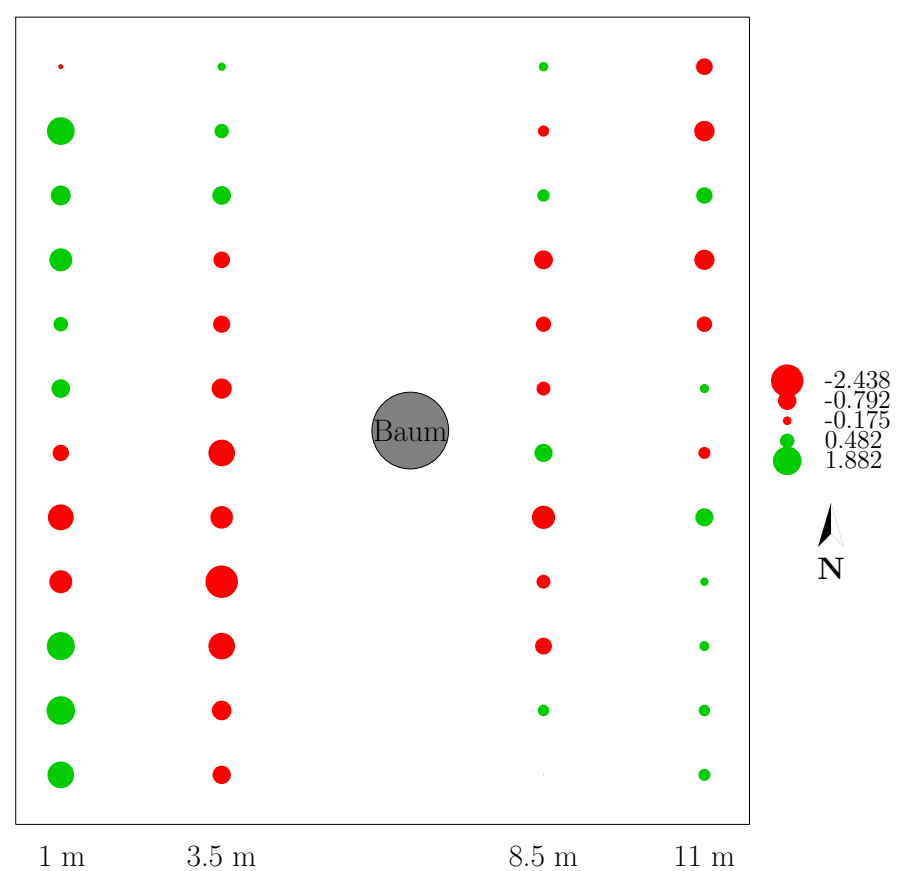

b) Verteilung der Residuen auf der Messfläche.

Abbildung 4.27: Statistische Auswertung der Beprobungen auf der Messfläche Schwarzkiefer. 


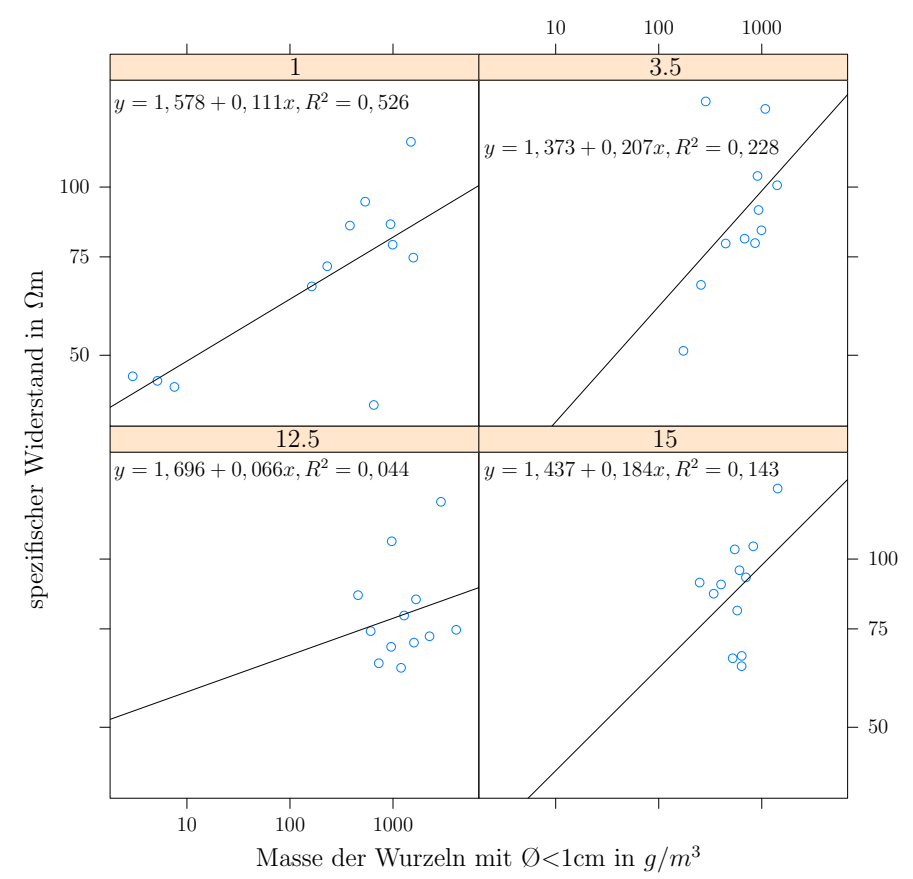

a) Zusammenhang zwischen spezifischem Widerstand und Wurzelmasse.

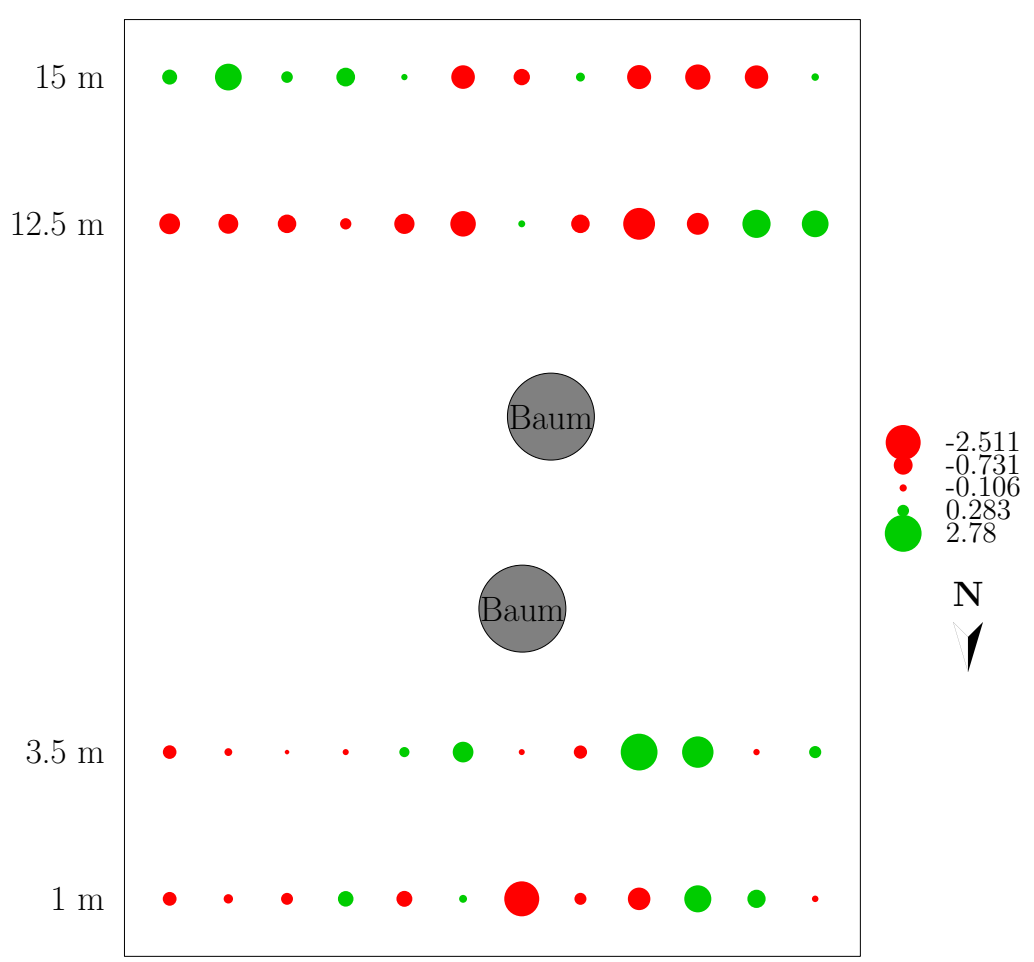

b) Verteilung der Residuen auf der Messfläche.

Abbildung 4.28: Statistische Auswertung der Beprobungen auf der Messfläche Buchengruppe. 


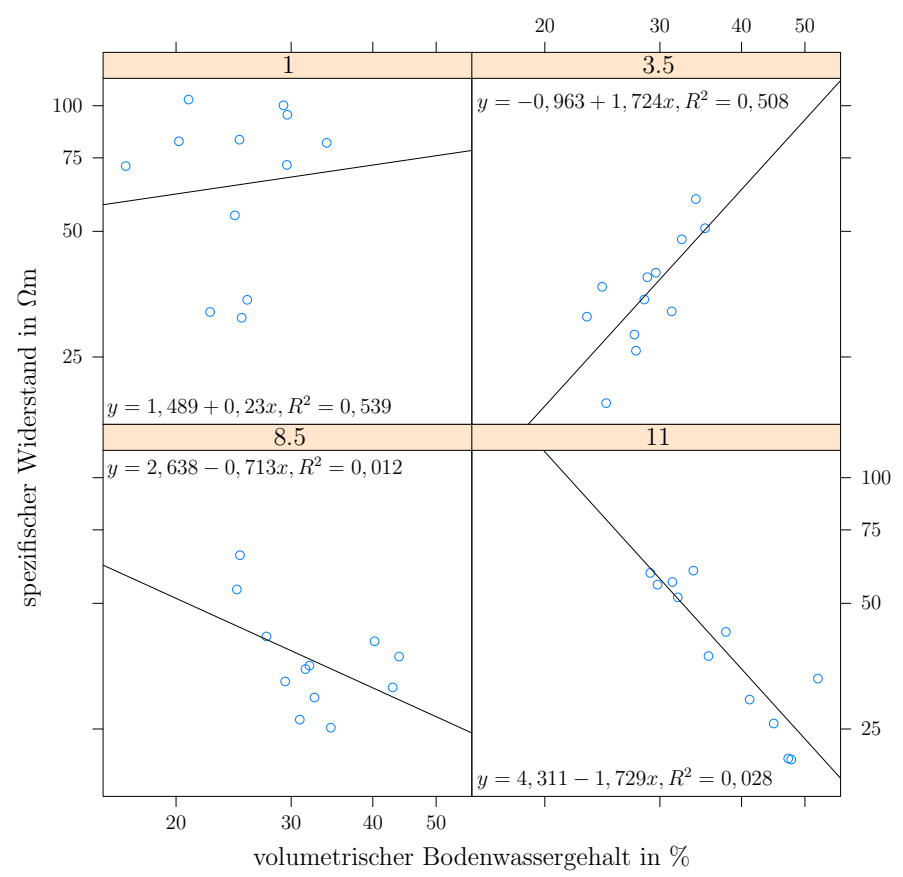

a) Schwarzkiefer

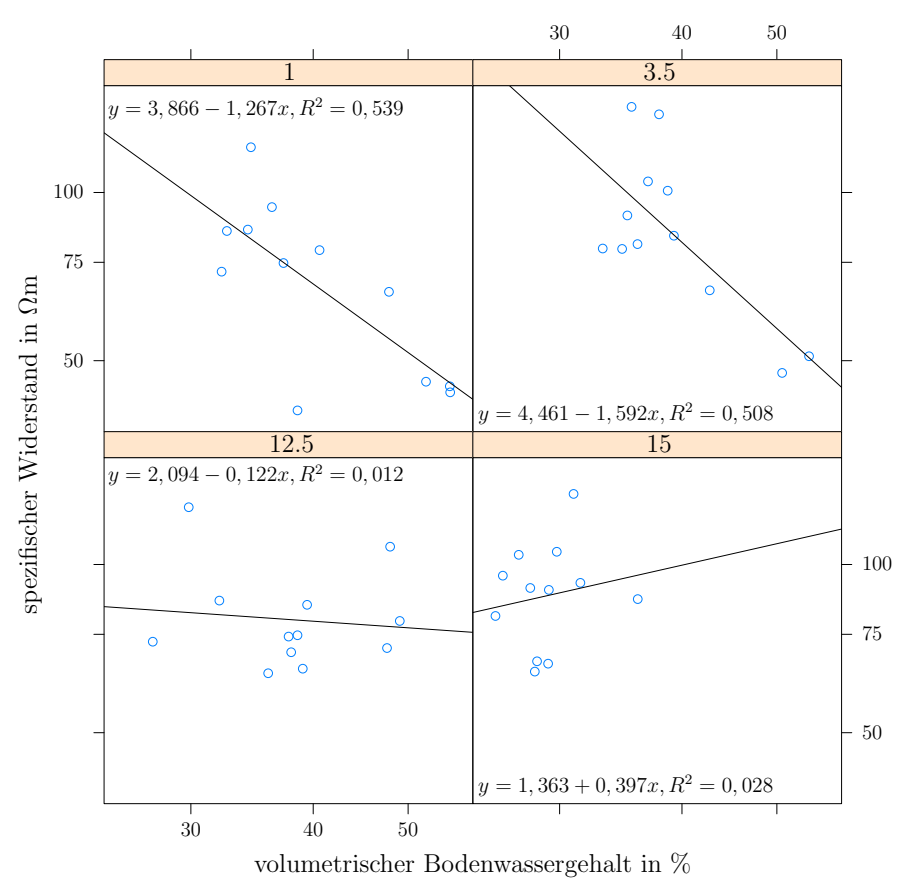

b) Buchengruppe

Abbildung 4.29: Zusammenhang zwischen spezifischem Widerstand und volumetrischem Bodenwassergehalt auf den Messflächen Schwarzkiefer und Buchengruppe. 


\subsubsection{Zusammenfassung der grundlegenden Ergebnisse}

Aufbauend auf den in den Versuchsbecken erzielten Ergebnissen konnte der Einfluss verschiedener Faktoren auf die Ergebnisse dreidimensionaler Bodenradarmessungen ermittelt werden. Die Befahrung der Messfläche mit einem xy-Raster und orthogonal zueinander angeordneten Antennen erbringt demnach die besten Ergebnisse bezüglich der Antennenanordnung. Die Einbeziehung einer zusätzlichen Antennenanordnung bringt keine Verbesserung. Diagonal zu den Transekten verlaufende Objekte konnten deutlich abgebildet werden.

Bei der Wahl der Rasterdichte hat sich ein Abstand von 15,0 cm als sinnvoll erwiesen. Dieser liegt im Bereich der in den Lysimeterbecken ermittelten lateralen Auflösung und hat in der Praxis eine kontinuierliche Darstellung der Wurzelverläufe ermöglicht. Gröbere Abstände führen zu deutlichen Aufösungsverlusten bishin zur Unkenntlichkeit der Objekte.

Für die Visualisierung hat sich die in den Kapiteln 2.3.2.3 und 3.3.3 beschriebene Filterkombination bewährt. Insbesondere die Filter background removal und migration führen zu einer besseren Darstellung, wobei dies in Relation zu den Rohdaten zu betrachten ist. Bei sehr guten Rohdaten ist die Verbesserung verhältnismäßig gering.

Die Reproduzierbarkeit des Visualisierungsergebnisses konnte einerseits unter Einsatz derselben Mittenfrequenz gezeigt werden. Darüber hinaus konnten die Wurzeln auf einer mehrfach untersuchten Teilfläche der Schwarzkiefer auch mit einer höherfrequenten Antenne erneut detektiert werden. Das Ergebnis dieser eher orientierenden Messung zeigt, dass Wurzeln mit einer höheren Messfrequenz feiner dargestellt werden und deutet gleichzeitig an, dass die vermehrten Details auch zu Problemen bei der Visualisierung führen könnten, da hierdurch mehr isolierte Nebenobjekte im Hintergrund erfasst werden.

Die Untersuchungen zur Verifikation haben gezeigt, dass insbesondere auf den homogenen Standorten Lage und Verlauf von Anomalien ein sicheres Kriterium zur Identifikation von Wurzeln in Radardaten sind. Mit zunehmender Komplexität der Untergrundzusammensetzung wird die sichere Ansprache erschwert. Dabei konnten auf Untersuchungsflächen, bei denen in den zugehörigen Radargrammen keine Wurzeln identifiziert werden konnten, oberflächennah auch keine Wurzeln freigelegt werden. 


\subsection{D-Georadar- und Geoelektrikmessungen auf ausgewählten Standorten}

\subsubsection{Schillerwiese Göttingen}

Schwarzkiefer Abbildung 4.30a zeigt einen Ausschnitt aus der Untersuchungsfläche Schwarzkiefer, in dem die Wurzelverläufe des Baumes bis an den Rand der untersuchten Fläche nachvollzogen werden können. Die Identifikation von Wurzeln ist bei diesem Messbeispiel in dem begrenzten Laufzeitfenster $6 \mathrm{~ns}-10 \mathrm{~ns}$ möglich, was darauf hindeutet, dass die detektierbaren Wurzeln der Schwarzkiefer eine geringe vertikale Ausdehnung aufweisen. Im Zentrum der Horizontalschnitte, die das Wurzelsystem am besten visualisieren, befindet sich eine eher flächige Reflexion (Abbildung 4.30a $\bigcirc$ ), aus der einzelne Wurzelstränge mit deutlichen Verzweigungen und Richtungsänderungen (Abbildung 4.30a z.B. $\longrightarrow$ ) herausstreichen.

Ab einer Laufzeit von 10 ns setzen die Wurzelreflexionen weitgehend aus. Anstelle der Wurzeln tritt eine andere Struktur (Abbildung 4.30b zwischen _..-) in der Zeitscheibe auf, die auf eine in Baumnähe verlaufende Leitung hinweist. Lediglich an drei Stellen können noch wurzelartige Reflexionen zu späteren Laufzeiten gefunden werden (Abbildung $4.30 \mathrm{~b} \longrightarrow$ ).

Die Ergebnisse der Geoelektrikuntersuchung auf der selben Messfläche zeigen dagegen keine Anomalien, die dahingehend interpretiert werden können, dass sie dem Wurzelsystem der Schwarzkiefer entsprechen. Im oberflächennahen Tomogramm sind hochohmige Anomalien im Bereich des Baumes zu erkennen, die sich nach außen fortsetzen (Abbildung 4.31a innerhalb von O). Aber auch in den Ecken der Untersuchungsfläche liegen derartige hochohmige Anomalien in flächiger Ausprägung vor (Abbildung 4.31a ). Ihre Lage spricht nicht dafür, dass sie von den Baumwurzeln verursacht worden sind.

Ebenso verhält es sich mit Tomogrammen aus tieferen Bereichen. Hier liegen im westlichen Bereich der Messfläche gröbere hochohmige Strukturen vor, die aufgrund von Form und Lage nicht mit dem Wurzelwerk der Schwarzkiefer in Verbindung gebracht werden können (Abbildung 4.31b. Die auf der Messfläche verzeichneten Widerstandswerte liegen zwischen wenigen und $70 \Omega \mathrm{m}$.

Fichtengruppe Die Eignung der Schillerwiese für Radarmessungen zur Wurzeldetektion wird durch die Untersuchungsergebnisse auf der Messfläche Fichtengruppe bestätigt.

In der abgebildeten Ergebniszeitscheibe sind die einzelnen Wurzelstränge der drei Bäume deutlich zu erkennen (Abbildung 4.32a z.B. $\longrightarrow$ ).

Im Vergleich zur Schwarzkiefer setzen die Wurzeln nach einer kürzeren Laufzeit ein und können mit leichten Veränderungen in der Form über eine längere Vertikaldistanz beziehungsweise Laufzeit verfolgt werden. Wesentliche morphologische Merkmale zur Identifikation der Wurzeln sind wie bei der Schwarzkiefer die Verbindung der genannten Anomalien zum Baum und die zunehmende Verzweigung nach außen.

Auch in den Ergebnissen der Geoelektrikuntersuchungen auf dieser Messfläche lassen sich Anomalien finden, die wahrscheinlich auf die Wurzelsysteme der Fichten zurückzuführen sind. Hier liegen in den obersten Tomogrammen hochohmige Bereiche vor, die unmittelbar an den drei Fichten auf der Messfläche beginnen. Während diese in unmittelbarer Nähe zum Baum flächig 


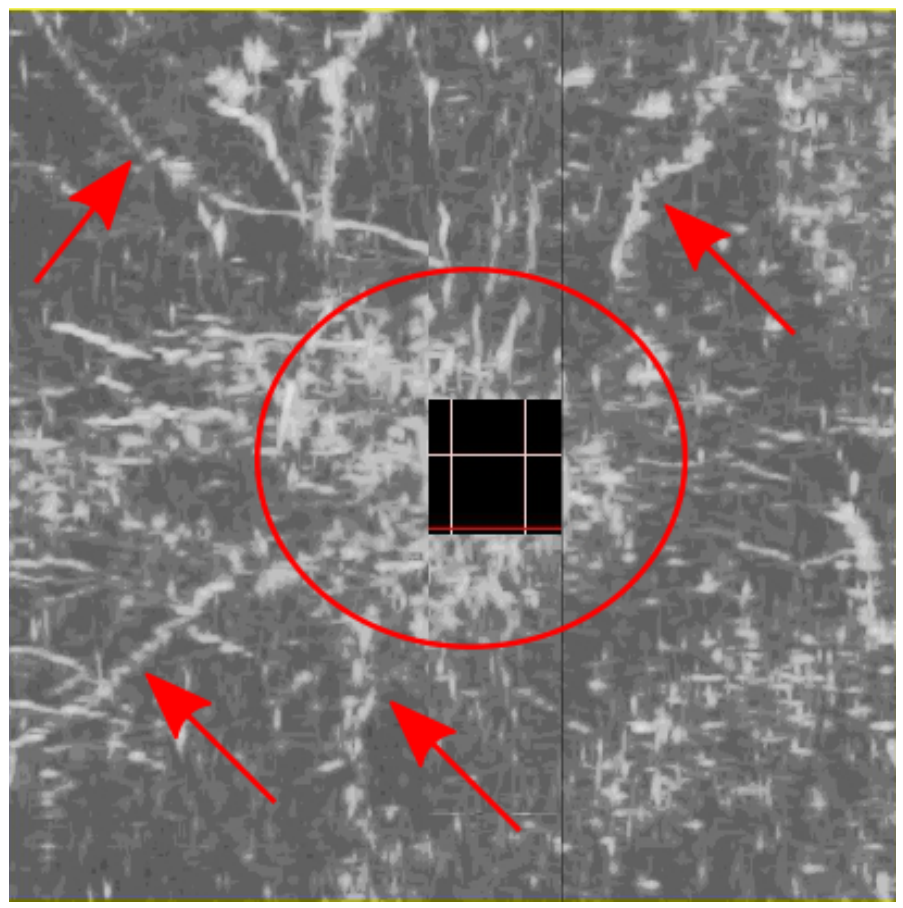

a) Die Parameter z-Wert $=8$; width $=3 ; v=0,07 \mathrm{~m} \mathrm{~s}^{-1} ; \varepsilon_{r}=18,36$ ergeben einen Tiefenbereich von $22,75 \mathrm{~cm}-33,25 \mathrm{~cm}$.

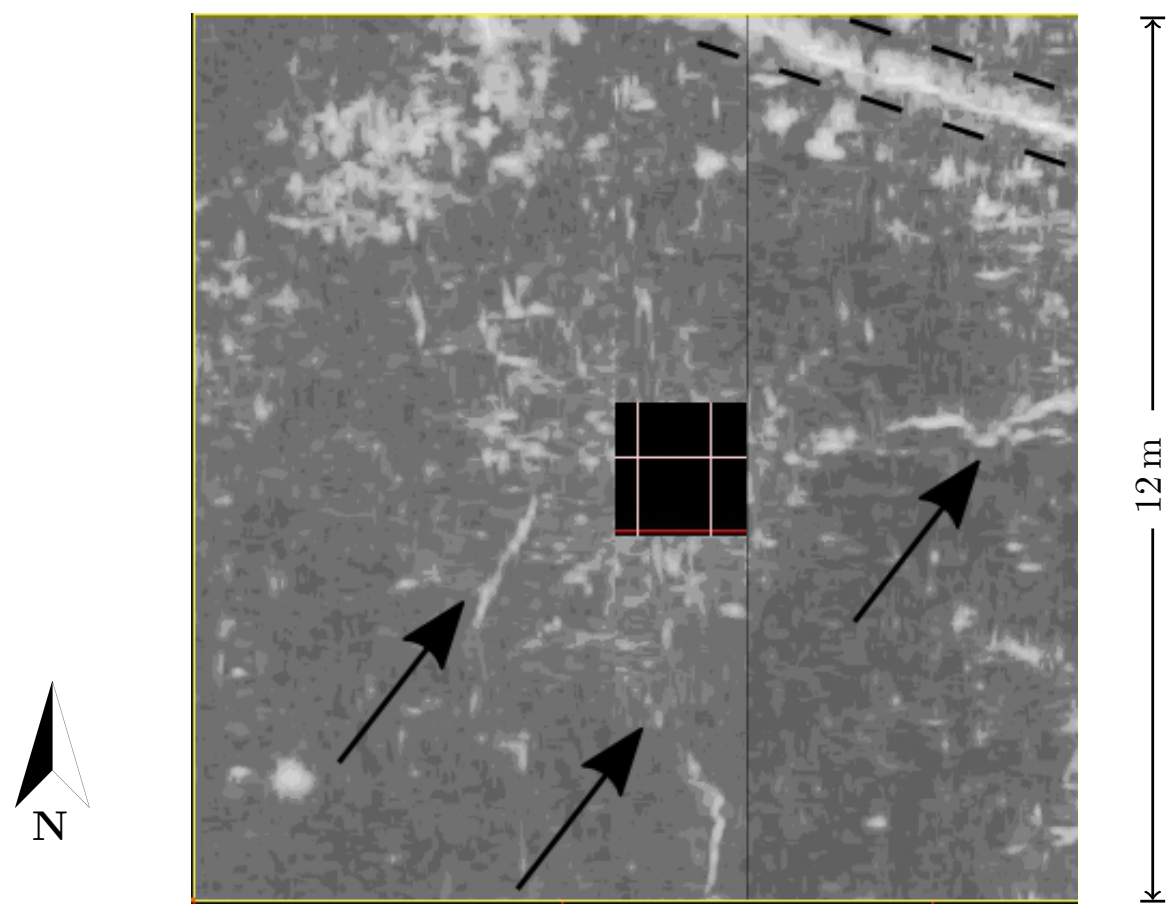

b) Die Parameter z-Wert $=14$; width $=4 ; v=0,055 \mathrm{~m} \mathrm{~s}^{-1} ; \varepsilon_{r}=20,66$ ergeben einen Tiefenbereich von $0,33 \mathrm{~cm}-0,44 \mathrm{~cm}$.

Abbildung 4.30: Georadaruntersuchung auf der Messfläche Schwarzkiefer in Form von ausgewählten Zeitscheiben. 

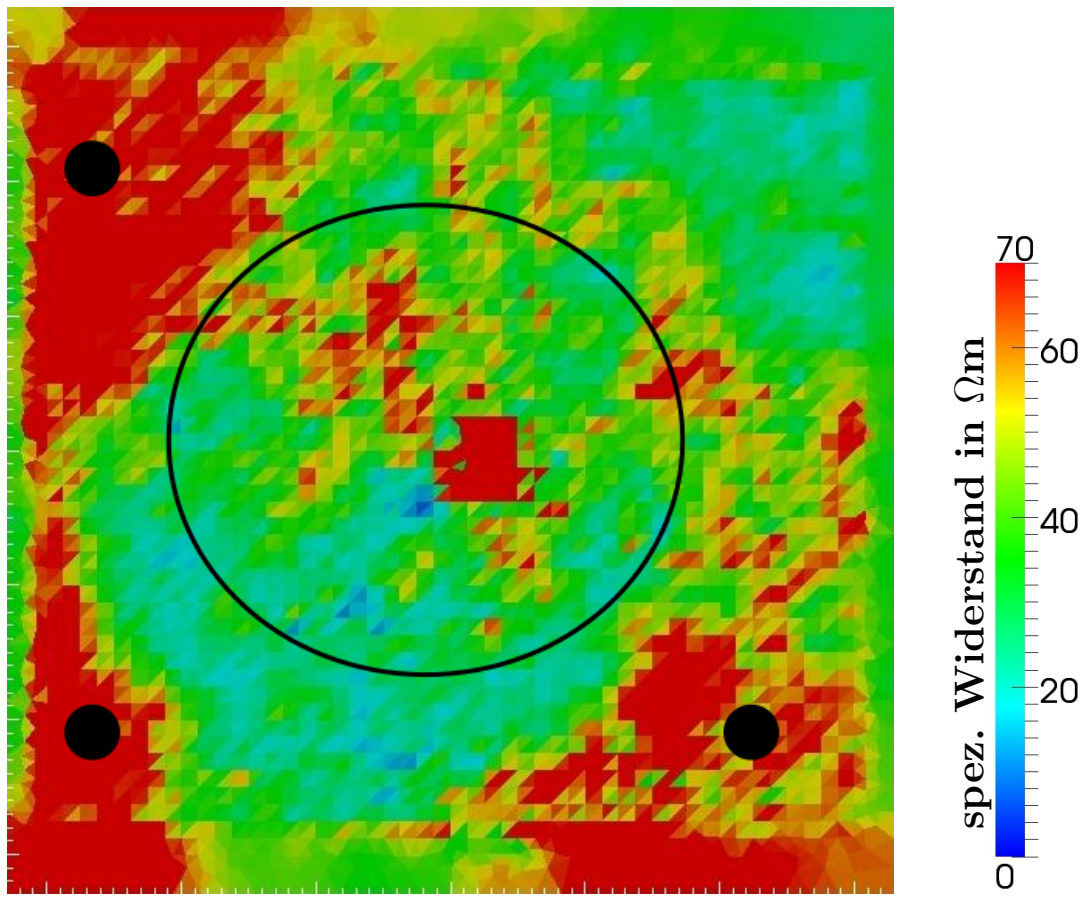

a) $0,1 \mathrm{~m}$ Tiefe
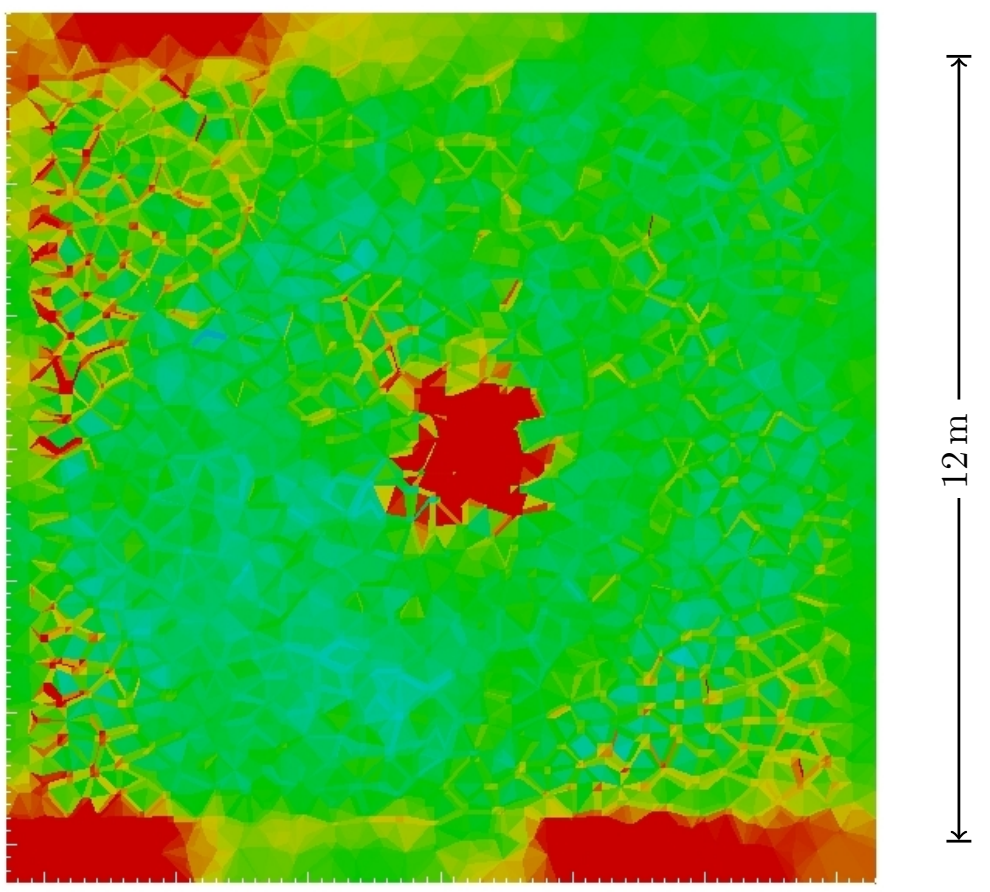

b) $0,4 \mathrm{~m}$ Tiefe

Abbildung 4.31: Geoelektrikuntersuchung auf der Messfläche Schwarzkiefer in Form von ausgewählten Horizontalschnitten. 


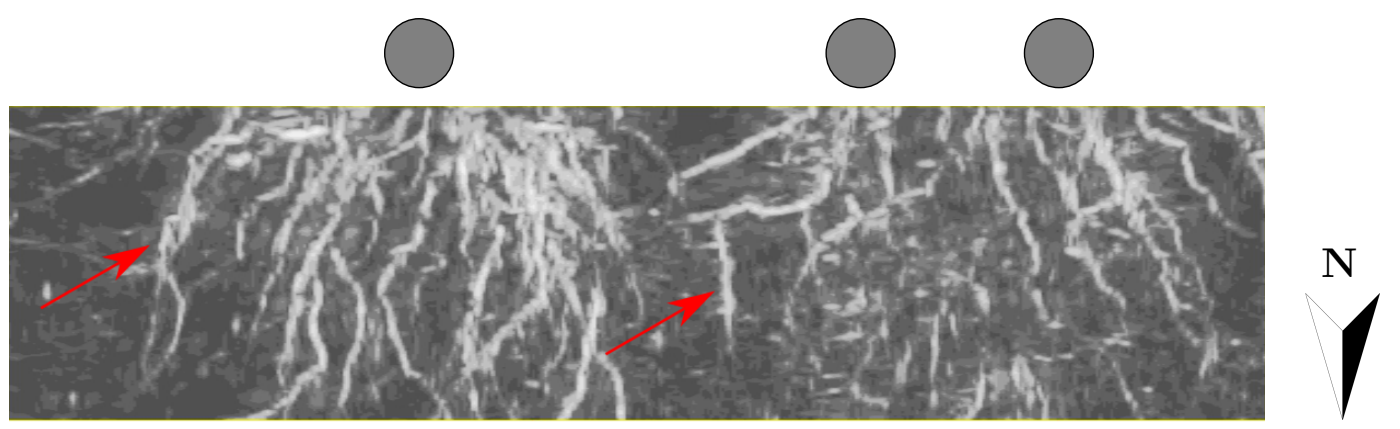

a) Die Parameter z-Wert $=4$; width $=4 ; v=0,1 \mathrm{~m} \mathrm{~ns}^{-1} ; \varepsilon_{r}=9$ ergeben einen Tiefenbereich von $10,0 \mathrm{~cm}-30,0 \mathrm{~cm}$.

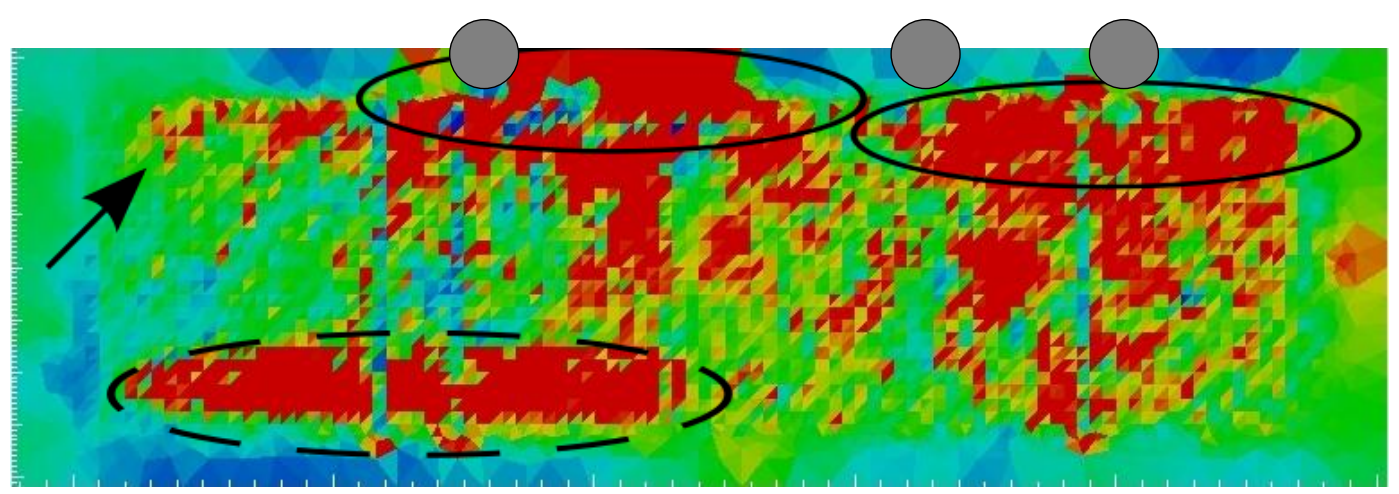

b) oberflächennah (Inversion weist $0 \mathrm{~m}$ Tiefe aus)
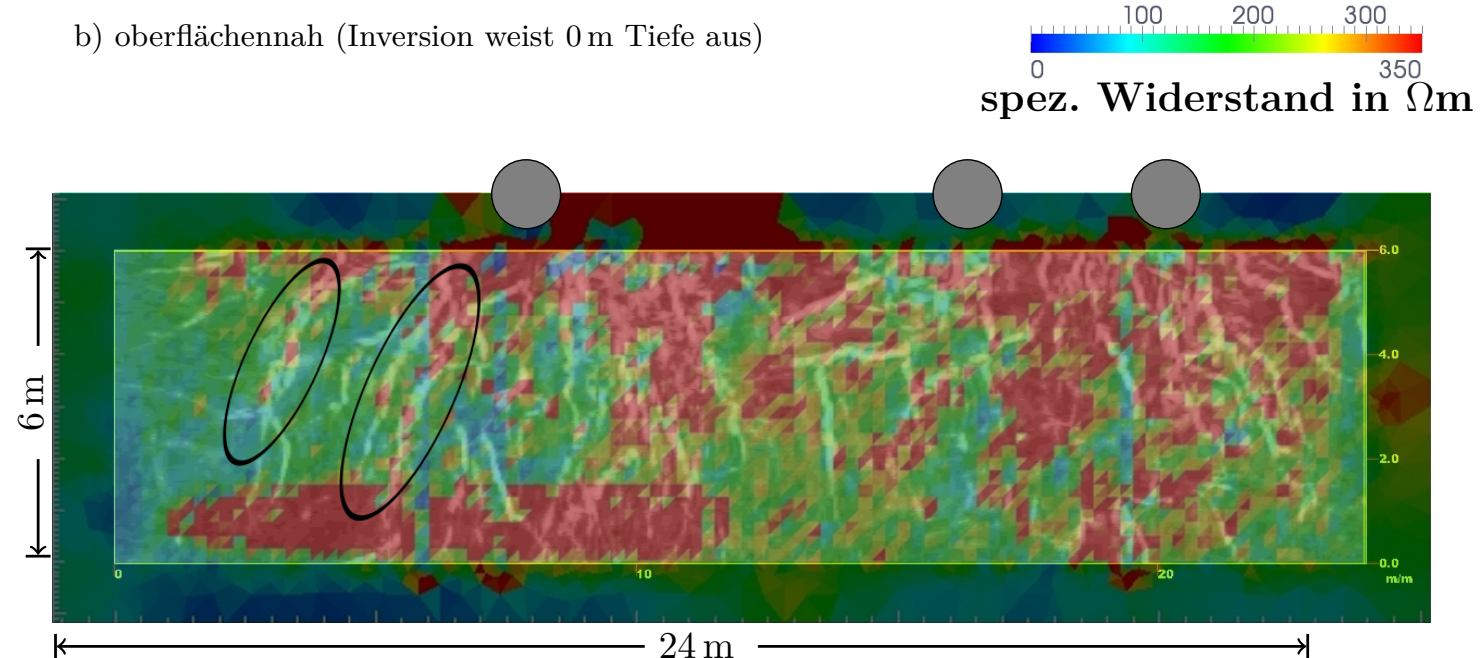

c) Überlagerung von Radargramm und Tomogramm

Abbildung 4.32: Ergebnis der Georadar- und Geoelektrikuntersuchungen auf der Messfläche Fichtengruppe in Form einer ausgewählten Zeitscheibe $(z$-Wert $=4$; width $=4)$ und eines ausgewählten Tomogramms an der Oberfläche (Inversion weist $0 \mathrm{~m}$ Tiefe aus), sowie einer Überlagerung von Zeitscheibe und Tomogramm. Die Tiefenumrechnung der GPR-Daten mit den Parametern z-Wert $=4$; width $=4 ; v=0,1 \mathrm{~m} \mathrm{~ns}^{-1} ; \varepsilon_{r}=9$ ergibt einen Tiefenbereich von $10,0 \mathrm{~cm}-30,0 \mathrm{~cm}$. 
ausgeprägt sind (Abbildung $4.32 \mathrm{~b} \bigcirc$ ), werden sie an der Peripherie deutlich filigraner und gabeln sich teilweise auf (Abbildung $4.32 \mathrm{~b}$ z.B. $\longrightarrow$ ).

Daneben existiert eine parallel zur Längsseite verlaufende Anomalie in der unteren linken Ecke der obersten Tomogramme (Abbildung $4.32 \mathrm{~b}(-$ - '). Diese verläuft parallel zur Querseite und kann nicht mit dem Wurzelsystem der Fichte in Verbindung gebracht werden. Bei diesem Messbeispiel reichen die Widerstände von sehr niedrigen Werten bis etwa $300 \Omega \mathrm{m}$ und übertreffen damit die Widerstandswerte auf der Messfläche Schwarzkiefer.

Die Überlagerung von Radargramm und Tomogramm zeigt, dass Bereiche, in denen die hochohmigen Anomalien im Tomogramm sich in einzelne filigrane Bereiche auflösen, in vielen Fällen deckungsgleich mit den im Radargramm identifizierten Wurzelsträngen sind (z.B. Abbildung $4.32 \mathrm{c} O$ ).

Fichte Bei der solitären Fichte fallen die Messergebnisse wiederum anders aus. In den oberflächennahen Radargrammen können Teile des Wurzelsystems durch die GPR-Messung visualisiert werden. Die Wurzeln können insbesondere in der unteren linken Ecke bis zum Bildrand nachvollzogen werden (Abbildung 4.33a innerhalb $\bigcirc$ ).

Auch in anderen Bildteilen lassen sich Wurzeln vom Stamm bis zum Bildrand verfolgen (Abbildung 4.33a $\longrightarrow$ ), wobei Zwischenbereiche aber Lücken oder schwächere Amplituden aufweisen (z.B. Abbildung 4.33a innerhalb ':'). Neben den Wurzeln ist auch ein unbekanntes, rundes Objekt in der Zeitscheibe vorhanden (Abbildung 4.33a innerhalb '

Etwas tiefer (z-Wert 10; width 4) sind zwei lineare, auf Leitungen hinweisende Strukturen zu erkennen (Abbildung 4.33b zwischen _.-- ). Der Laufzeitbereich, in dem diese Strukturen auftreten, liegt dabei mit etwa $8 \mathrm{~ns}$ bis $12 \mathrm{~ns}$ etwas niedriger als bei der Leitung im Messfeld Schwarzkiefer. Die umgerechneten Tiefen liegen mit Werten von 0,33 cm-0,44 cm (Schwarzkiefer) $38,0 \mathrm{~cm}-57,0 \mathrm{~cm}$ (Fichte) in ähnlichen Bereichen.

Die oberflächennahen Geoelektrikmessungen im Bereich der Fichte weisen in Baumnähe hochohmige Anomalien auf (Abbildung 4.34a innerhalb $\mathrm{O}$ ), die sich ähnlich wie im Fall der Fichtengruppe in einzelne, filigrane Objekte auflösen, dabei aber nicht die Auflösung der Radarmessung erreichen. Bis auf den nördlichen Rand weisen alle Ränder der Messfläche ebenfalls hochohmige Anomalien auf, die im Gegensatz zu den beschriebenen, baumnahen Strukturen flächig ausfallen (Abbildung 4.34a ).

In dem Tomogramm aus einer Tiefe von 0,6 m befindet sich in der südöstlichen Ecke eine hochohmige Anomalie (Abbildung 4.34b zwischen _.-- ), deren Lage und Verlauf sich mit Lage und Verlauf der Leitung deckt, die sich im Radargramm abzeichnet. Im Vergleich zum Radargramm fehlt aber die zweite Leitung in der südwestlichen Ecke des Tomogramms. Mit Werten von wenigen bis zu $48 \Omega \mathrm{m}$ wurden hier die niedrigsten Widerstandswerte auf der Schillerwiese gemessen.

Buchengruppe Die Bodenradaruntersuchungen auf der Messfläche Buchengruppe fallen nicht so eindeutig wie die bisherigen Beispiele aus. Oberflächennah sind zahlreiche ungeordnete Reflexionen vorhanden. Hier liegt eine hohe Reflexionsdichte im Zentrum der Messfläche im Bereich um die beiden Bäume (Abbildung 4.35a $\mathrm{O}$ ) und in den Randbereichen (Abbildung 4.35a ('-) vor. 


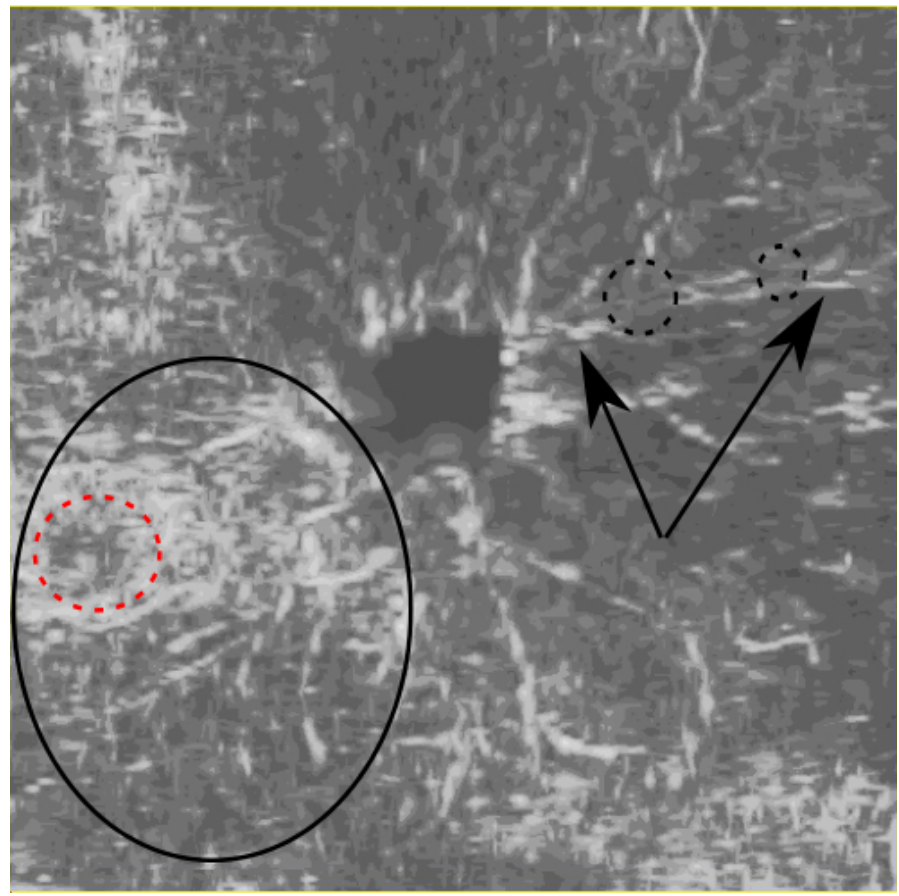

a) Die Parameter z-Wert $=4$; width $=4 ; v=0,12 \mathrm{~m} \mathrm{~ns}^{-1} ; \varepsilon_{r}=6,25$ ergeben einen Tiefenbereich von $12,0 \mathrm{~cm}-36,0 \mathrm{~cm}$.

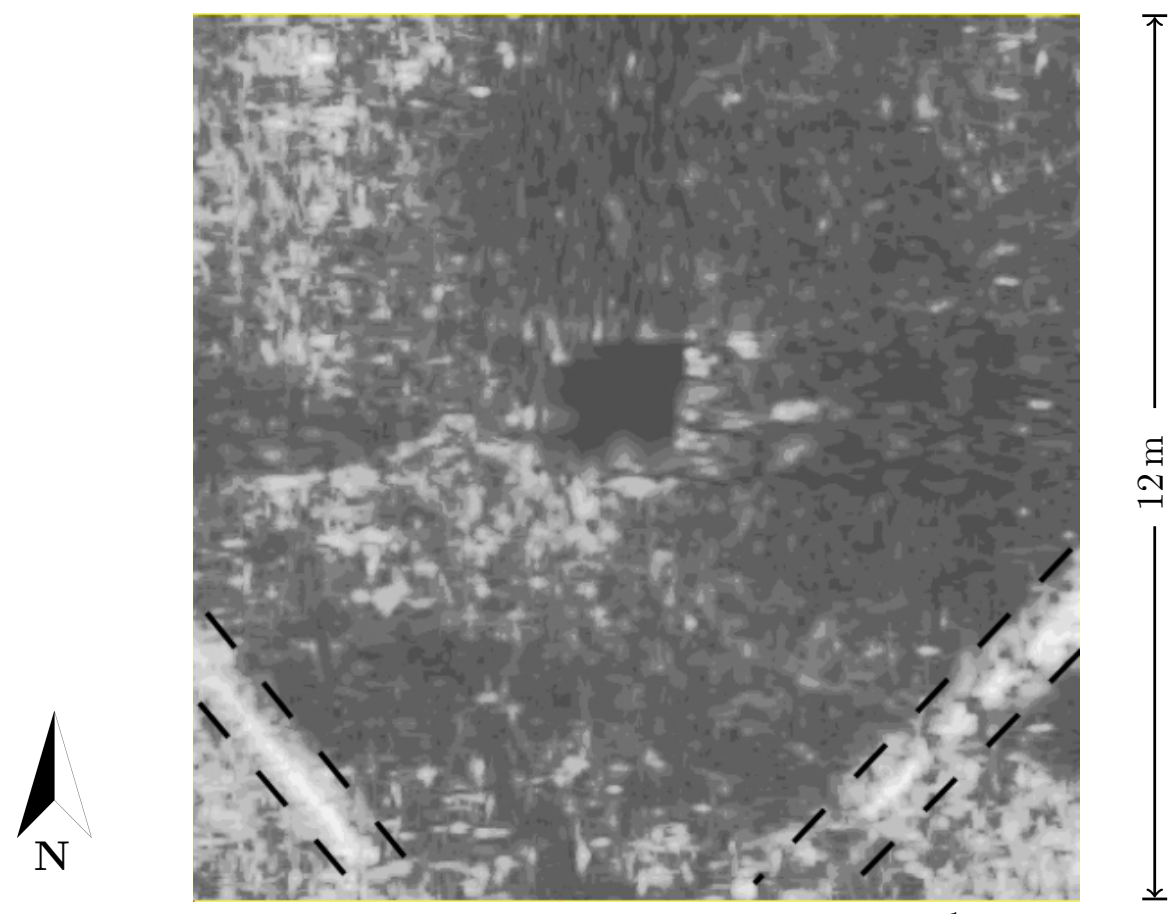

b) Die Parameter z-Wert $=10$; width $=4 ; v=0,095 \mathrm{~m} \mathrm{~ns}^{-1} ; \varepsilon_{r}=9,97$ ergeben einen Tiefenbereich von $38,0 \mathrm{~cm}-57,0 \mathrm{~cm}$.

Abbildung 4.33: Georadarmessung auf der Messfläche Fichte in Form ausgewählter Zeitscheiben. 


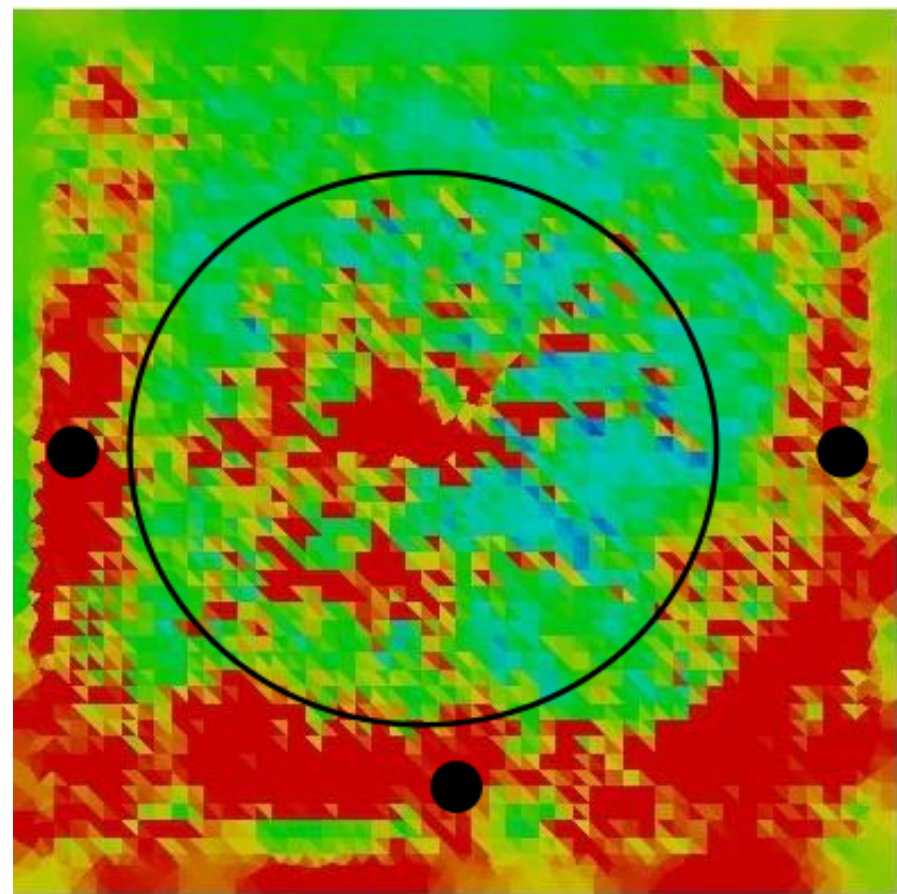

a) oberflächennah (Inversion weist eine Tiefe von $0 \mathrm{~m}$ aus)

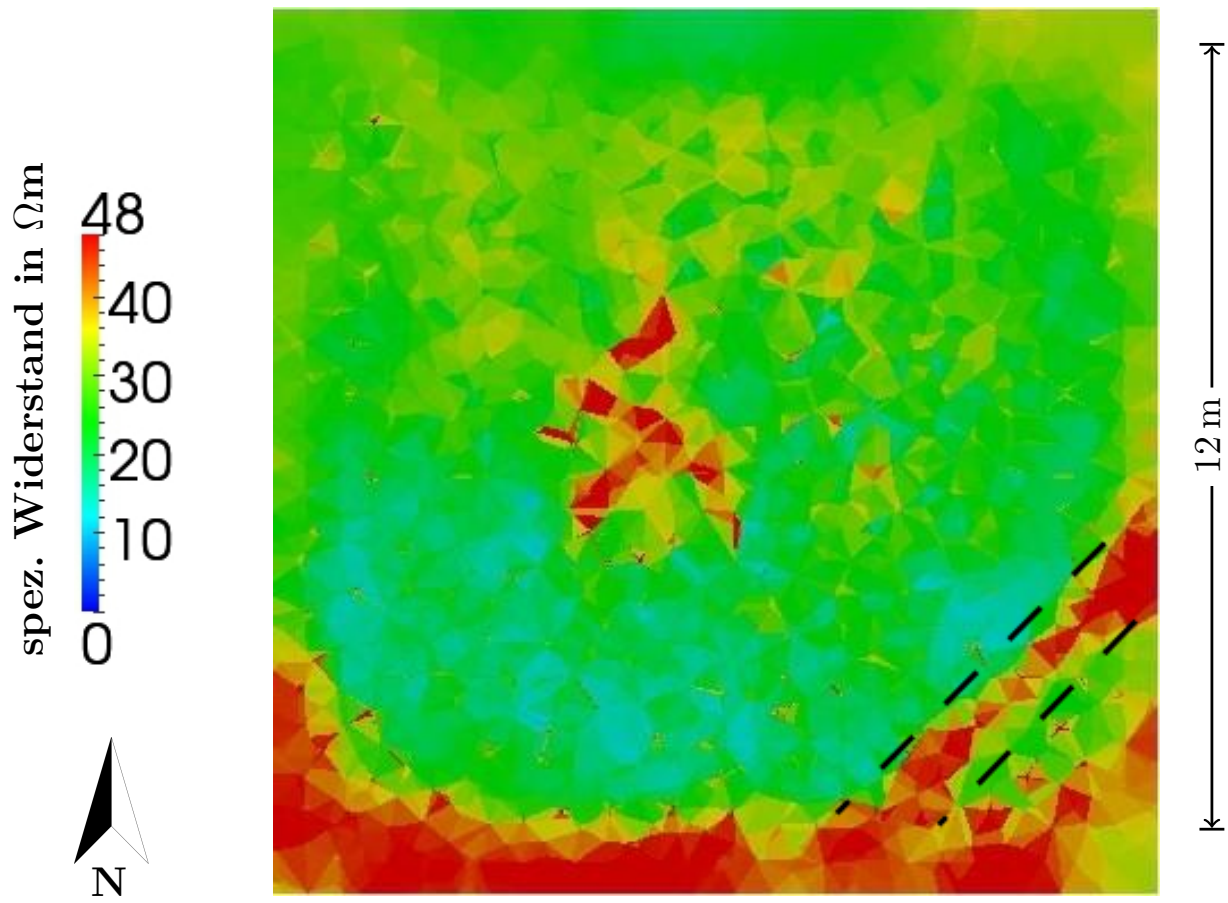

b) $0,6 \mathrm{~m}$ Tiefe

Abbildung 4.34: Geoelektrikmessung auf der Messfläche Fichte in Form von ausgewählten Horizontalschnitten. 
Die Reflexionen in der Peripherie verteilen sich sowohl auf Areale, die an Bäume außerhalb der Messfläche angrenzen, als auch auf Bereiche, in deren Nähe keine Bäume vorhanden sind. Des Weiteren liegt eine dreieckige Anomalie in der nordöstlichen Ecke der Messfläche vor (Abbildung $4.35 \mathrm{a} \nabla)$.

Mit zunehmender Tiefe können drei Klassen von Reflexionen in den Horizontalschnitten gebildet werden. In Bereichen, in denen sich Bäume befinden, werden filigrane auf Wurzeln hindeutende Strukturen sichtbar (Abbildung 4.34b $\longrightarrow$ ), in der nordwestlichen Ecke ist weiterhin die dreieckige Fläche (Abbildung $4.35 \mathrm{~b} \nabla$ ) zu erkennen und ein lineares, auf eine Leitung hinweisendes Objekt befindet sich nahe des linken Bildrandes Abbildung 4.35b zwischen ..- -). Die Leitung tritt zu einer geringeren Laufzeit als die Leitungen in den anderen Untersuchungsbeispielen (Schwarzkiefer und Fichte) auf der Schillerwiese auf. Die Tiefenumrechnung ergibt für diesen Fall eine absolute Tiefe zwischen $13,0 \mathrm{~cm}-26,0 \mathrm{~cm}$.

Die für die Buchengruppe erstellten Widerstandstomogramme weisen hochohmige Anomalien auf, die darauf schließen lassen, dass durch sie die Wurzeln der Bäume auf (Abbildung 4.36a ○) und am Rand außerhalb der Messfläche (Abbildung $4.36 \mathrm{a}\left(\mathrm{C}^{\prime}\right.$ ') visualisiert werden. Bei den randlichen Anomalien fällt auf, dass diese an den westlichen und südlichen Bildrändern vorhanden sind, an denen sich auch jeweils Bäume befinden. Dagegen liegen im Bereich der östlichen und nördlichen Messflächenränder, an denen sich keine Bäume befinden, niedrigere Widerstände vor. Die in den Radargrammen identifizierte, dreieckige Fläche im nordöstlichen Bildrand kann auch in den Tomogrammen als niederohmige Anomalie wiedergefunden werden (Abbildung 4.35a $\square$ ). Insgesamt wurden an der Buchengruppe Widerstände zwischen wenigen und $400 \Omega \mathrm{m}$ gemessen.

Die Überlagerung von Radargramm und Tomogramm (Abbildung 4.36b zeigt keine deutliche Kongruenz zwischen Reflexionen und hochohmigen Anomalien, wobei die laterale Ausdehnung der jeweiligen Bereiche ähnlich ist.

Zusammenfassung der Befunde auf der Schillerwiese Durch die Georadar- und Geoelektrikmessungen auf der Schillerwiese konnten die Wurzelsysteme der untersuchten Bäume stellenweise oder zu wesentlichen Teilen visualisiert werden. Die Ergebnisse der Radarmessungen erzielen dabei eine bessere Auflösung als die Geoelektrikmessungen und konnten in allen Fällen Teile des jeweiligen Wurzelsystems visualisieren, während die Geoelektrikmessungen nur im Fall der Fichtengruppe eindeutige Ergebnisse lieferten. Neben den Primärzielen Baumwurzeln konnten an verschiedenen Stellen auch Fremdobjekte wie Leitungen deutlich visualisiert und anhand von Form und Lage von den Wurzeln abgegrenzt werden. 


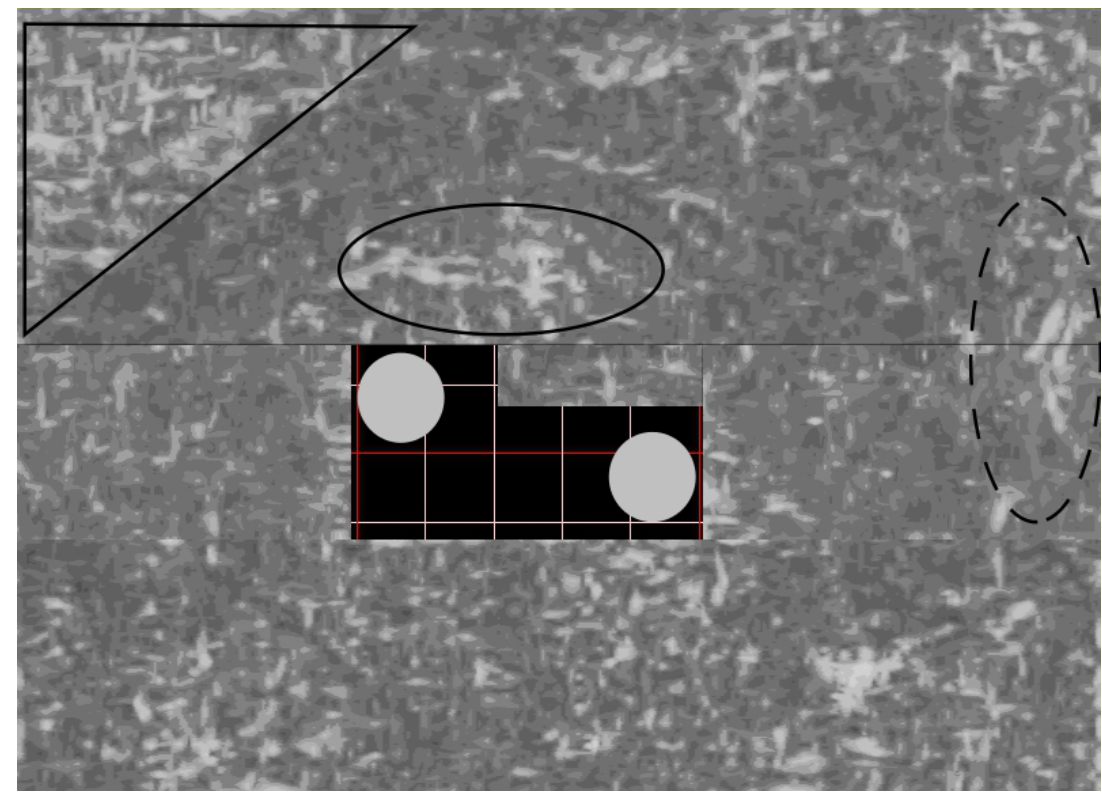

a) Die Parameter z-Wert $=2$; width $=2 ; v=0,065 \mathrm{mns}^{-1} ; \varepsilon_{r}=21,3$ ergeben einen Tiefenbereich von $3,25 \mathrm{~cm}-9,75 \mathrm{~cm}$.

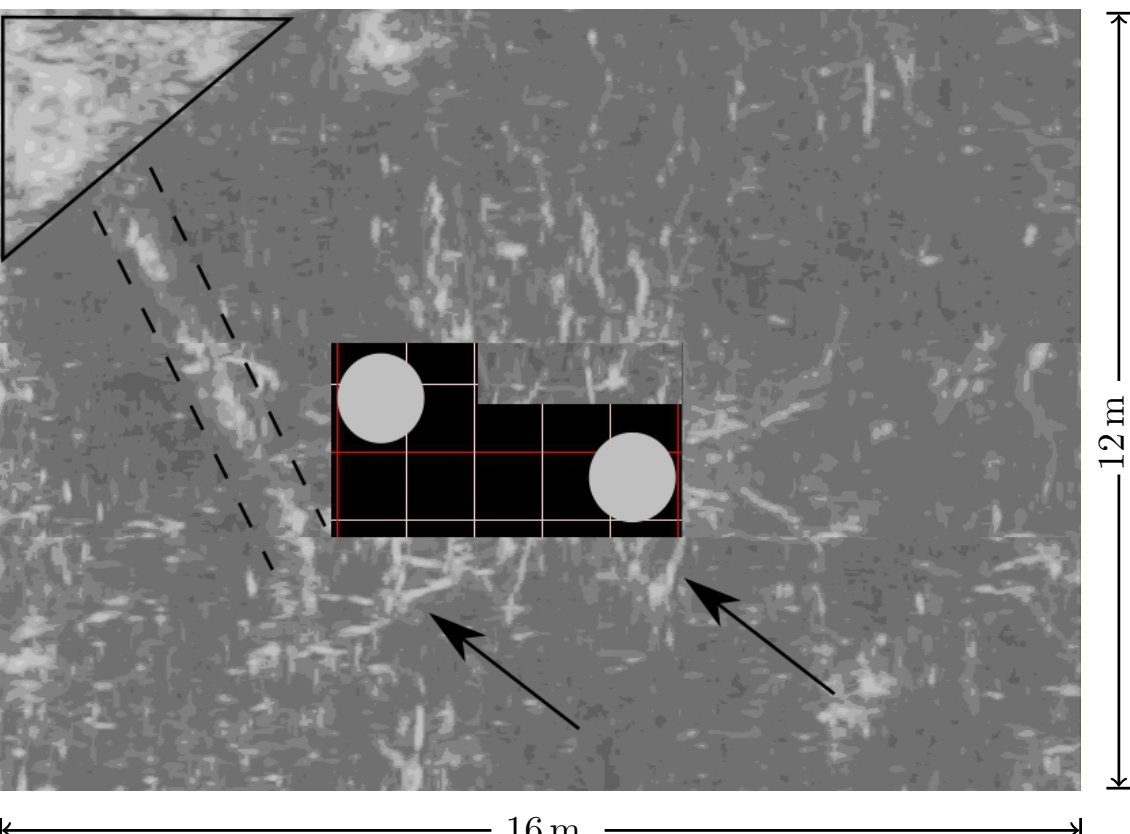

b) Die Parameter z-Wert $=6$; width $=4 ; v=0,065 \mathrm{~m} \mathrm{~ns}^{-1} ; \varepsilon_{r}=21,3$ ergeben einen Tiefenbereich von $13,0 \mathrm{~cm}-26,0 \mathrm{~cm}$.

Abbildung 4.35: Georadarmessung auf der Messfläche Buchengruppe in Form von ausgewählten Zeitscheiben. 

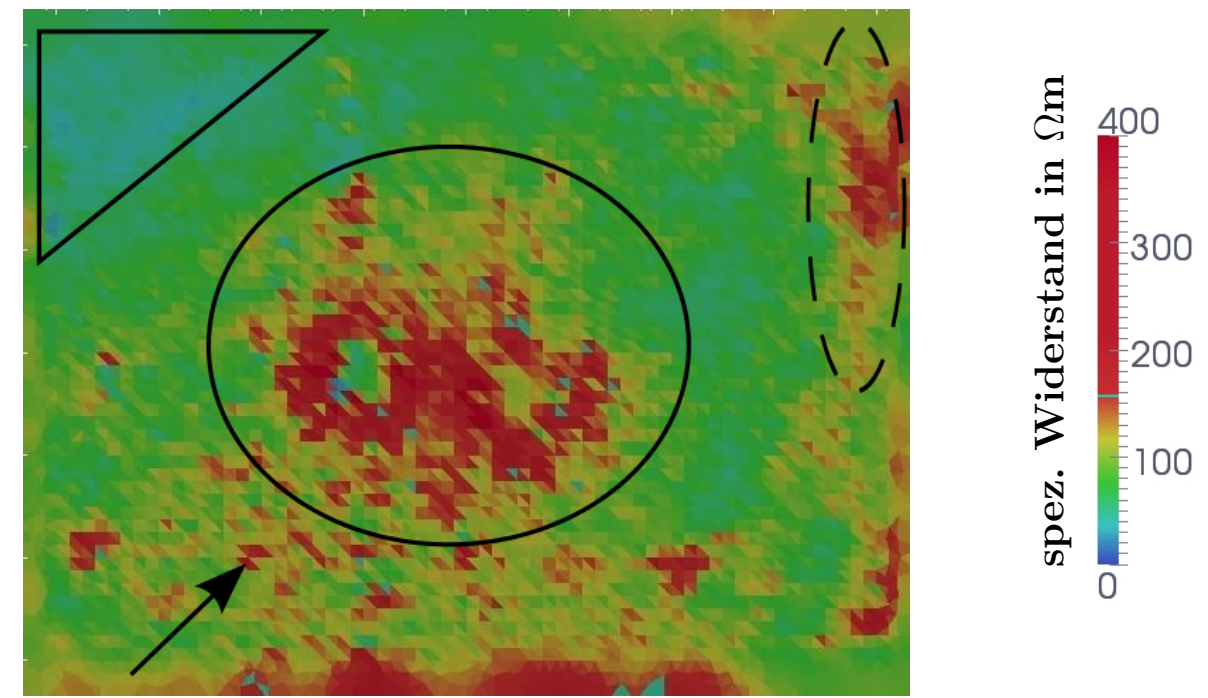

a) oberflächennah (Inversion weist eine Tiefe von $0 \mathrm{~m}$ aus)

$\mathbf{N}$

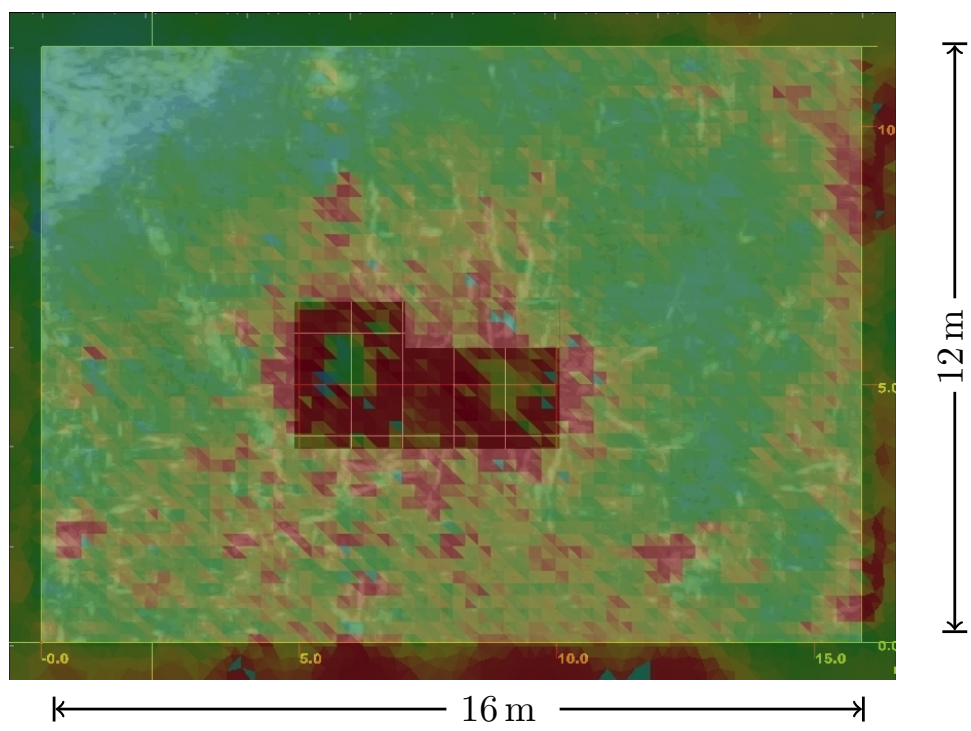

b) Überlagerung von GPR und ERT. Für das GPR ergeben die Parameter $\mathrm{z}$-Wert $=6$; width $=4 ; v=0,12 \mathrm{~m} \mathrm{~ns}^{-1} ; \varepsilon_{r}=6,25$ einen Tiefenbereich von $12,0 \mathrm{~cm}-36,0 \mathrm{~cm}$.

Abbildung 4.36: Ergebnis der Geoelektrikuntersuchung auf der Messfläche Buchengruppe in Form eines ausgewählten Horizontalschnitts (Tiefe $0 \mathrm{~m}$ ) und einer Überlagerung von Radar- und Geoelektrikergebnis. 


\subsubsection{Landwehrkanal Berlin}

Silberahorn Das erste Untersuchungsbeispiel am Berliner Landwehrkanal ist ein Silberahorn am Einsteinufer. In den resultierenden Radargrammen liegen deutliche Reflexionen vor, deren Form und Lage darauf hinweisen, dass hier das Wurzelsystem des Baumes erfasst worden ist (Abbildung $4.37 \mathrm{a}$ z.B. $\longrightarrow$ ). Die Anomalien liegen in einer hohen Dichte vor, dabei können aber immer noch einzelne Wurzelstränge unterschieden werden. Die Dichte dieser oberflächennah ausgeprägten Reflexionen nimmt ab einer Laufzeit von etwa 6 ns deutlich ab, so dass keine weiteren Wurzeln ab dieser Tiefenstufe identifiziert werden können.

Die Widerstandstomogramme auf dieser Messfläche weisen mit bis zu $2800 \Omega \mathrm{m}$ deutlich höhere Messwerte als die auf der Schillerwiese auf. Die höchsten erfassten Widerstände werden dabei in Baumnähe verzeichnet, während in den Randbereichen niedrigere Widerstände vorliegen. Auffällig ist dabei, dass diese hochohmigen Anomalien eher kleinräumig, fleckig vorliegen und kein geschlossenes größeres Areal wie bei den Messflächen auf der Schillerwiese einnehmen (Abbildung 4.37b).

Die Überlagerung von Radargramm und Tomogramm zeigt keine deutliche Kongruenz zwischen den Ergebnissen. In den Bodenradarmessungen identifizierte Reflexionen verteilen sich gleichermaßen auf nieder- und hochohmige Bereiche.

Hybridpappel In den Messergebnissen der Radaruntersuchung an der Hybridpappel lassen sich bis zu einer Laufzeit von $6 \mathrm{~ns}$ Indikationen für Teile des Wurzelsystems finden. Auffällig sind die Strukturen im rechten Teil der Radargramme, die ohne offensichtliche Verbindung zum Baum aus Richtung der Ufermauer in die Messfläche laufen (Abbildung 4.38a $\longrightarrow$ ). Die in Kapitel 4.2.6.1 gezeigten Aufgrabungen bestätigen die Vermutung, dass es sich hierbei um Baumwurzeln handelt. Die Dichte der im Radargramm identifizierten Wurzeln ist bei der Hybridpappel deutlich niedriger als in den Radargrammen der benachbarten Messfläche Silberahorn (Tabelle 3.1. Baum 3a).

Auch in den Widerstandstomogrammen lässt sich ähnlich wie beim Silberahorn eine Dominanz hochohmiger Areale im Zentrum der Messfläche beobachten, wobei die höchsten Widerstände im östlichen Teil der Messfläche liegen (Abbildung 4.38b). Auffällig ist dabei eine hochohmige Struktur, die etwa in dem Bereich verläuft, in dem die in den Radargrammen identifizierte Wurzel verläuft (Abbildung $4.38 \mathrm{c} \bigcirc$ ). Mit Werten zwischen wenigen und $3000 \Omega \mathrm{m}$ liegen die absoluten Werte der hier ermittelten spezifischen Widerstände in der Größenordnung der Messwerte beim benachbarten Silberahorn.

Große Kastanie Deutlich anders als am Einsteinufer fallen die Radargramme und Widerstandstomogramme auf den beiden Messflächen am Corneliusufer aus.

In den oberflächennahen Zeitscheiben der Messfläche Große Kastanie lassen sich keine eindeutigen, auf Wurzeln zurückzuführenden Reflexionen identifizieren. Stellenweise liegen lineare Strukturen vor. Verlauf und Form machen es aber unwahrscheinlich, dass diese von den Wurzeln des Baumes herrühren (Abbildung 4.39a $\longrightarrow$ ). So fallen einzelne Objekte sehr breit aus, haben keine eindeutige Verbindung zum Baum und weisen keine ausgeprägten Verzweigungen auf. Neben 


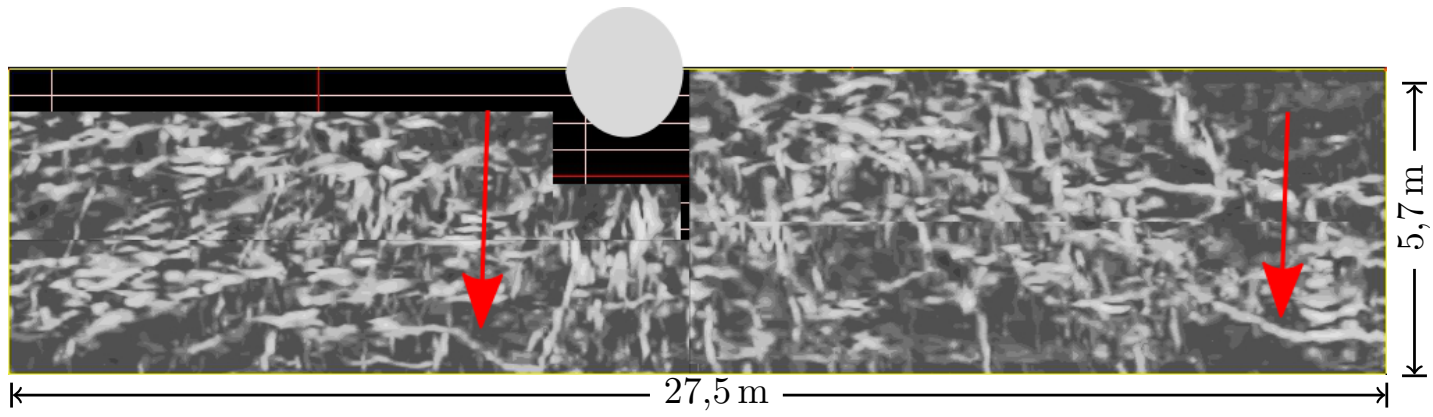

a) Die Parameter z-Wert $=2$; width $=1 ; v=0,1 \mathrm{~m} \mathrm{~ns}^{-1} ; \varepsilon=9$ ergeben einen Tiefenbereich von $7,5 \mathrm{~cm}-12,5 \mathrm{~cm}$.

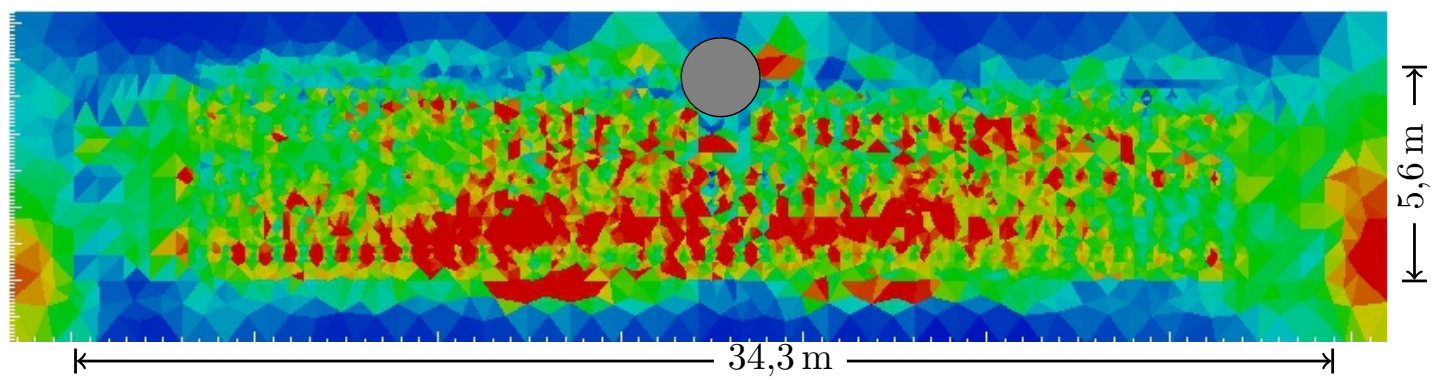

b) oberflächennah (Inversion weist eine Tiefe von $0 \mathrm{~m}$ aus)
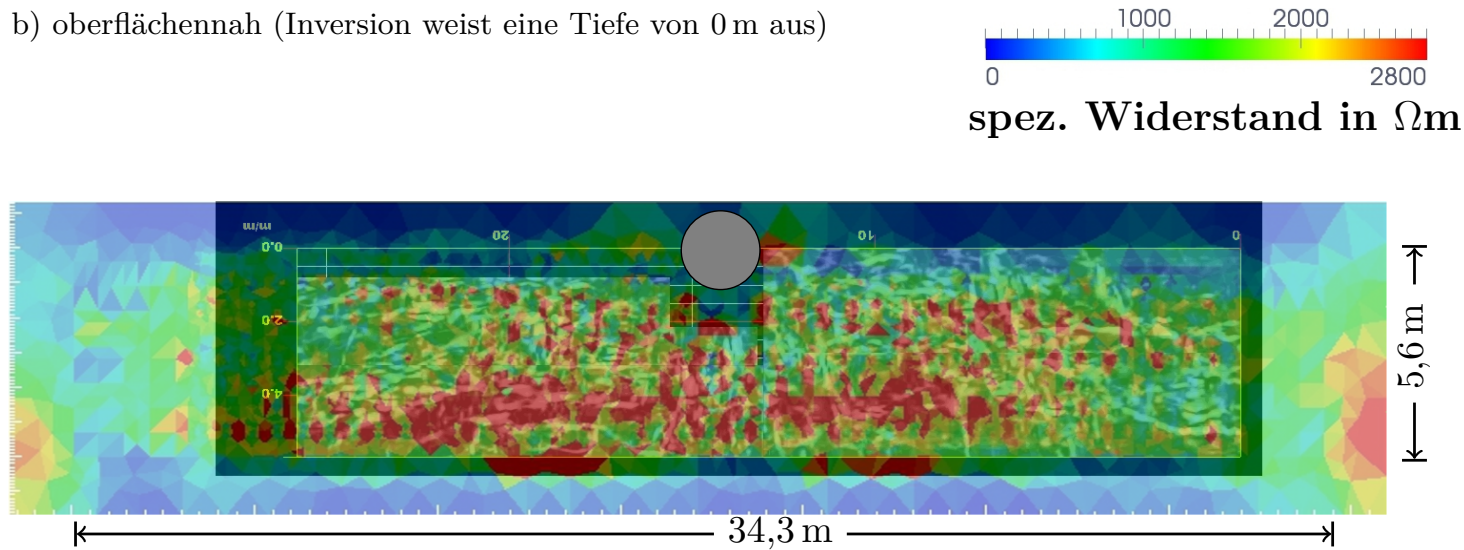

c) Überlagerung von Radargramm und Tomogramm

Abbildung 4.37: Ergebnis der Georadar- und Geoelektrikuntersuchungen auf der Messfläche Silberahorn in Form einer ausgewählten Zeitscheibe $(z-W e r t=2$; width $=1)$ und eines ausgewählten Tomogramms (0 m Tiefe), sowie einer Überlagerung von Zeitscheibe und Tomogramm. 


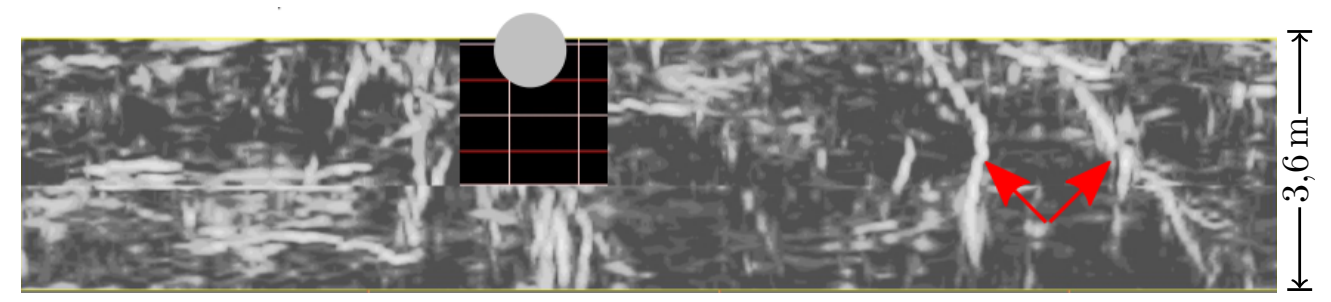

a) $\stackrel{\longleftrightarrow}{\text { Die Parameter z-Wert }=4 \text {; width }=3 ; v=0,18 \mathrm{~m} \mathrm{~ns}^{-1} ; \varepsilon_{r}=9 \text { ergeben }}$ einen Tiefenbereich von $12,5 \mathrm{~cm}-27,5 \mathrm{~cm}$.

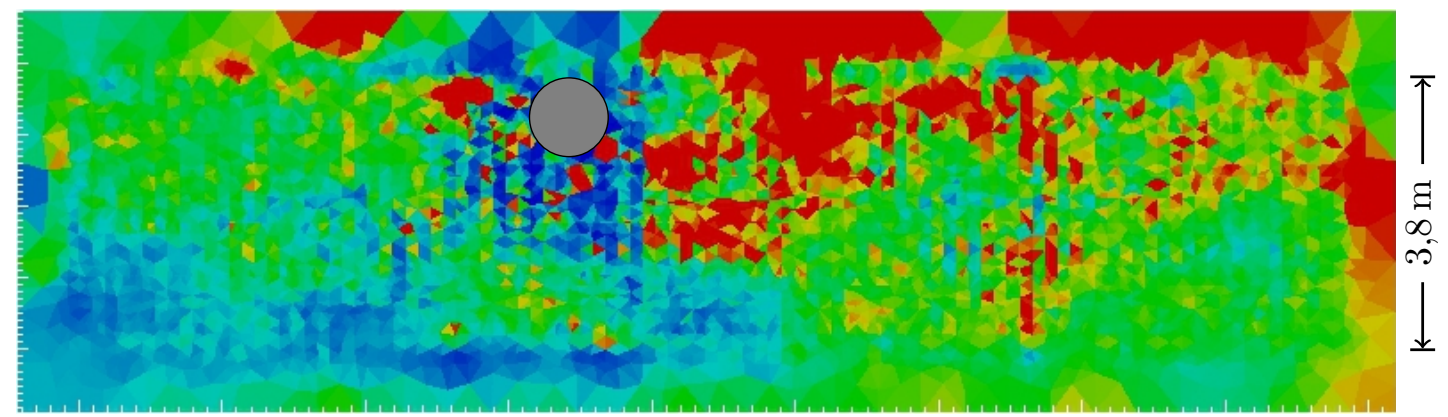

b) oberflächennah (Inversion weist eine Tiefe von $0 \mathrm{~m}$ aus)
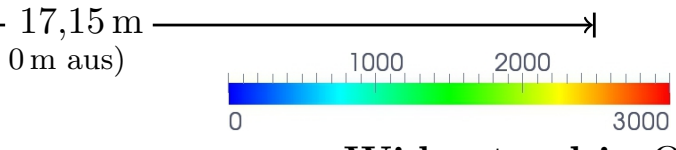

spez. Widerstand in $\Omega \mathrm{m}$

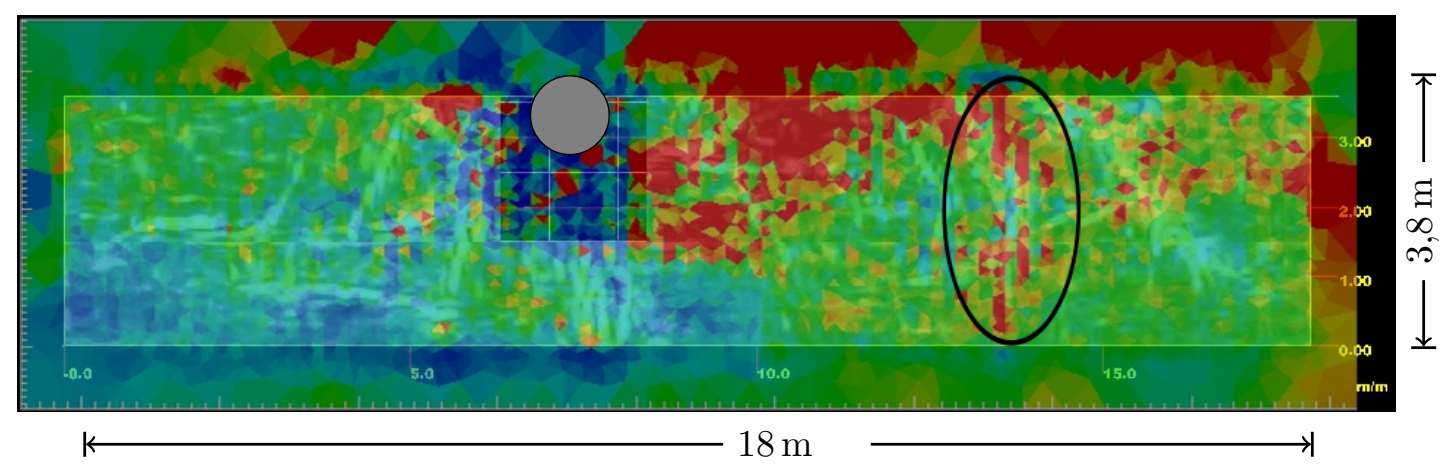

c) Überlagerung von Radargramm und Tomogramm

Abbildung 4.38: Ergebnis der Georadar- und Geoelektrikuntersuchunges auf der Messfläche Hybridpappel in Form einer ausgewählten Zeitscheibe $(\mathrm{z}$-Wert $=4$; width $=3$ ) und eines ausgewählten Tomogramms (Tiefe $0 \mathrm{~m}$ ), sowie einer Überlagerung von Zeitscheibe und Tomogramm. 


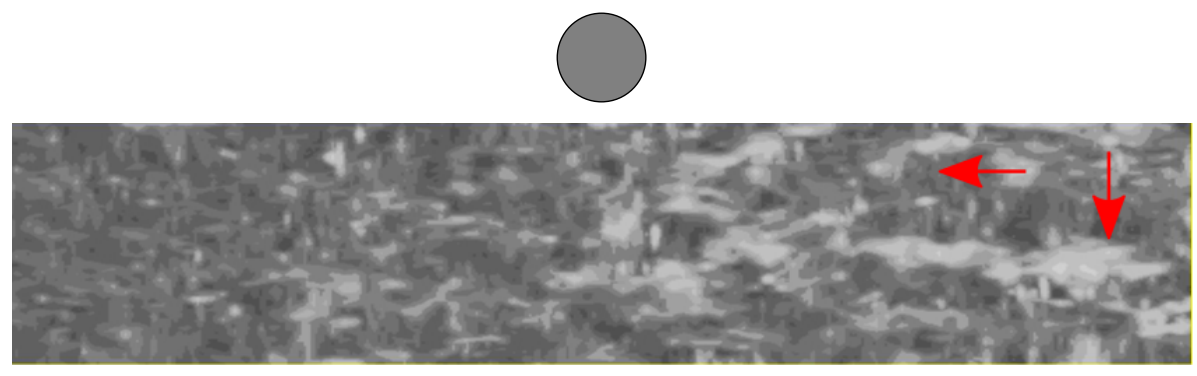

a) Die Parameter $\mathrm{z}$-Wert $=2$; width $=1 ; v=0,12 \mathrm{~m} \mathrm{~ns}^{-1} ; \varepsilon_{r}=6,25$ ergeben einen Tiefenbereich von $9,0 \mathrm{~cm}-15,0 \mathrm{~cm}$.
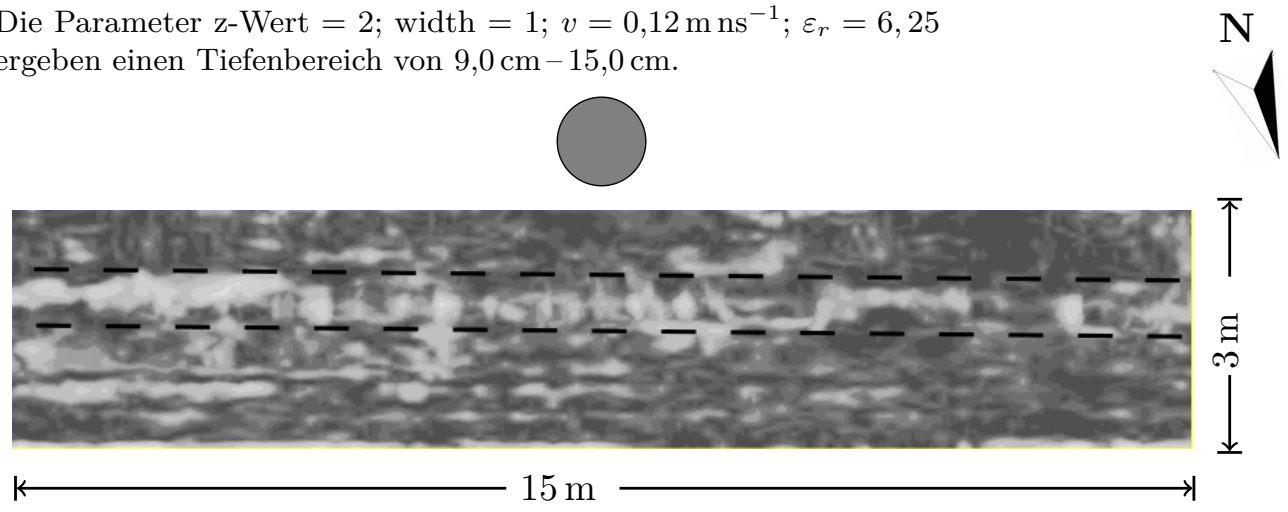

b) Die Parameter z-Wert $=14$; width $=4 ; v=0,075 \mathrm{~m} \mathrm{~ns}^{-1} ; \varepsilon_{r}=6,25$ ergeben einen Tiefenbereich von $45,0 \mathrm{~cm}-60,0 \mathrm{~cm}$.

Abbildung 4.39: Ergebnis der Georadaruntersuchung auf der Messfläche Große Kastanie in Form von ausgewählten Zeitscheiben.

diesen Reflexionen, die über Distanzen bis etwa 7,5 m verfolgt werden können, sind zahlreiche ungeordnete Anomalien in den Zeitscheiben zu finden.

In einem anderen Radargramm, das zu einer größeren Tiefenstufe gehört, kann ein lineares Objekt ausgemacht werden, das sich mittig parallel zur Längsseite erstreckt und wahrscheinlich zu einer Versorgungsleitung bzw. einem Kanal gehört (Abbildung 4.39b _..- ).

Die Tomogramme auf dieser Messfläche beinhalten, ähnlich wie die bisher analysierten, eine zentrale hochohmige Anomalie im Bereich des untersuchten Baumes, die sich in diesem Fall allerdings in sämtlichen Tiefenstufen finden lässt. Die horizontale Ausdehnung variiert dabei und erreicht in einer Tiefe zwischen 1,0 $\mathrm{m}$ und 1,4 m ihr Maximum. Die absoluten Widerstandswerte liegen zwischen sehr niedrigen und $980 \Omega \mathrm{m}$.

Kleine Kastanie Auch die Radargramme auf der benachbarten Messfläche Kleine Kastanie fallen nicht eindeutig in Bezug auf die Identifikation von Wurzeln aus. Oberflächennah ist eine flächige Anomalie (Abbildung 4.41a $\bigcirc$ ) ausgebildet und es lassen sich stellenweise fortlaufende lineare Objekte erkennen (Abbildung 4.41a $\longrightarrow$ ), die aus dieser hervorgehen. Daneben liegen auch bei dieser Messfläche zahlreiche, vom Baum isolierte Anomalien vor. Mit zunehmender Tiefe lassen sich dabei ebenfalls lineare Objekte (Abbildung 4.41b zwischen _. - -) beobachten, die wie beim Radargramm der Messfläche Große Kastanie parallel zur Längsseite der Messfläche verlaufen. 


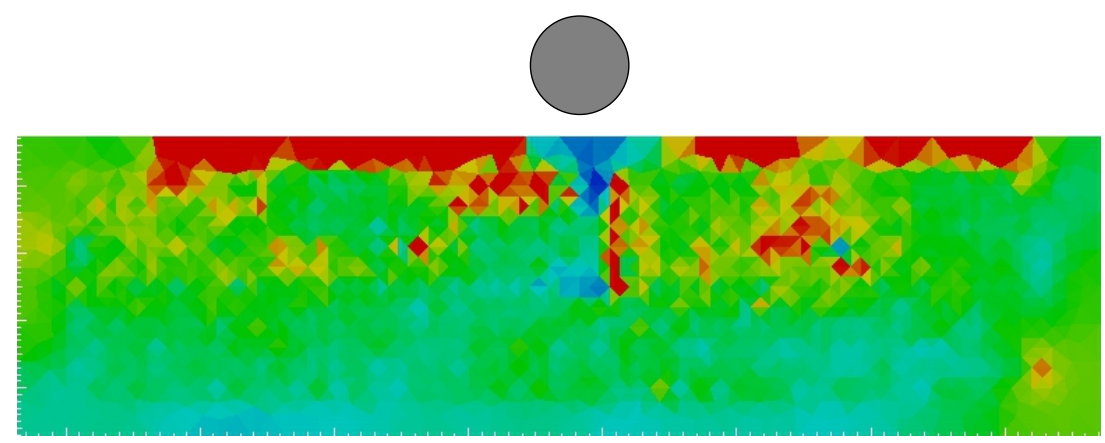

a) Tiefe $0 \mathrm{~m}$

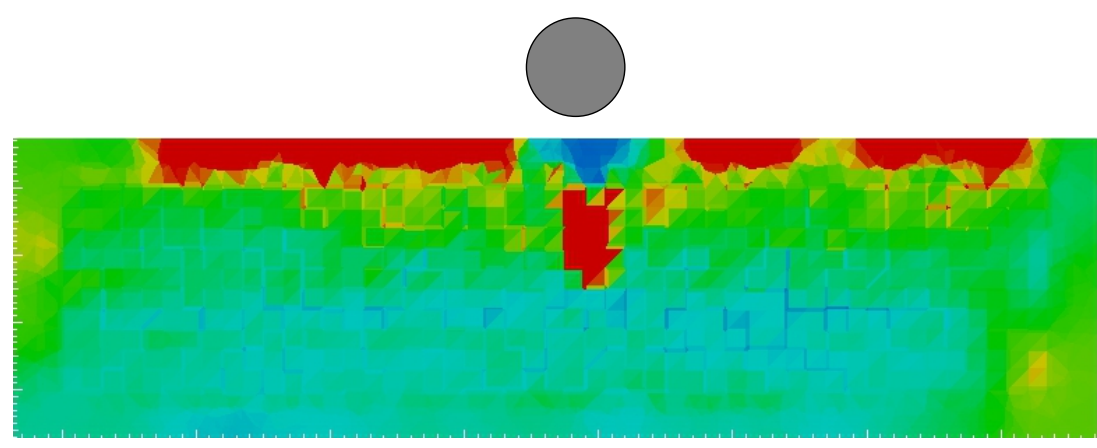

b) Tiefe $0,2 \mathrm{~m}$
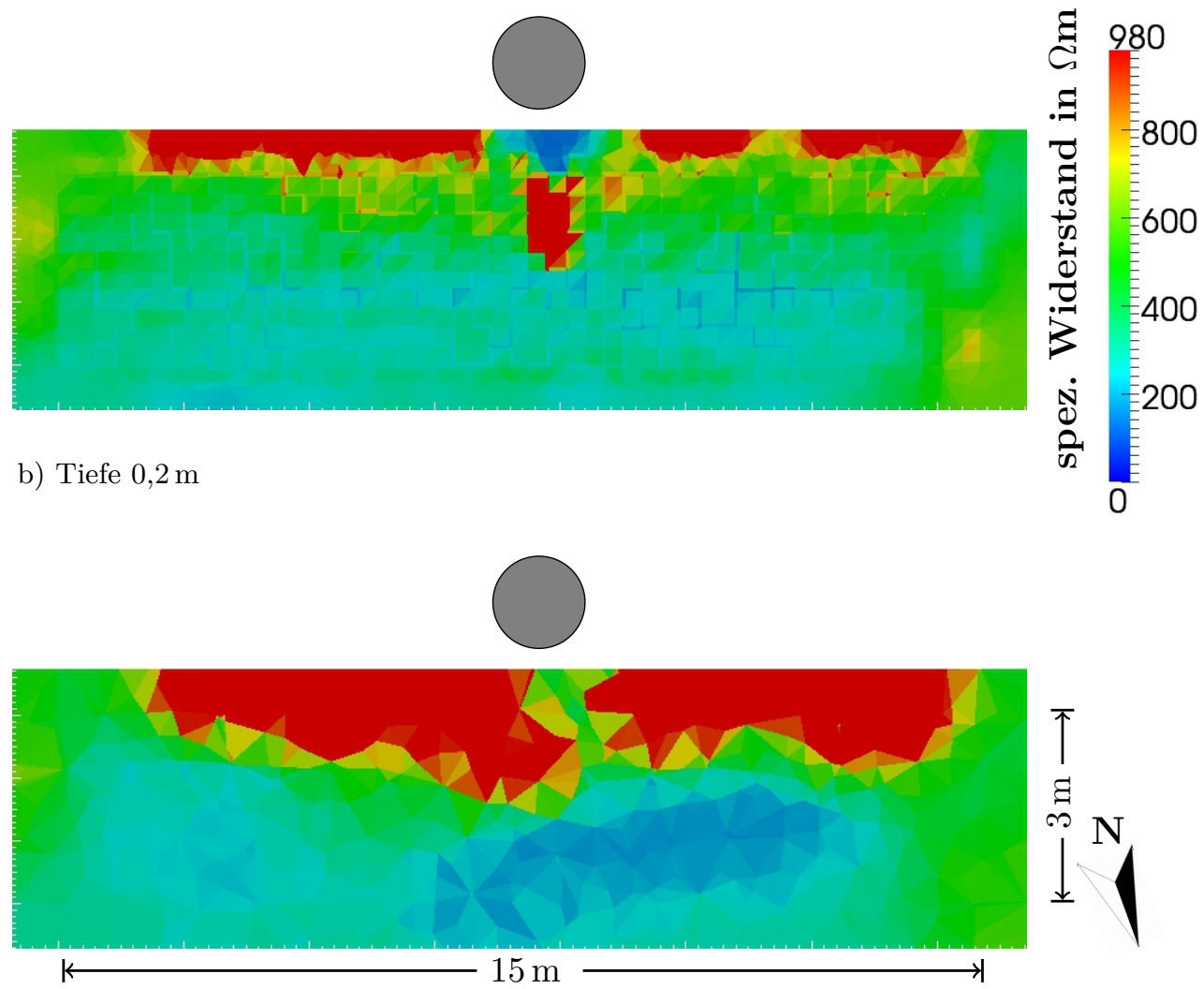

c) Tiefe $1,0 \mathrm{~m}$

Abbildung 4.40: Ergebnis der Geoelektrikuntersuchung auf der Messfläche Grosse Kastanie in Form von ausgewählten Horizontalschnitten. 


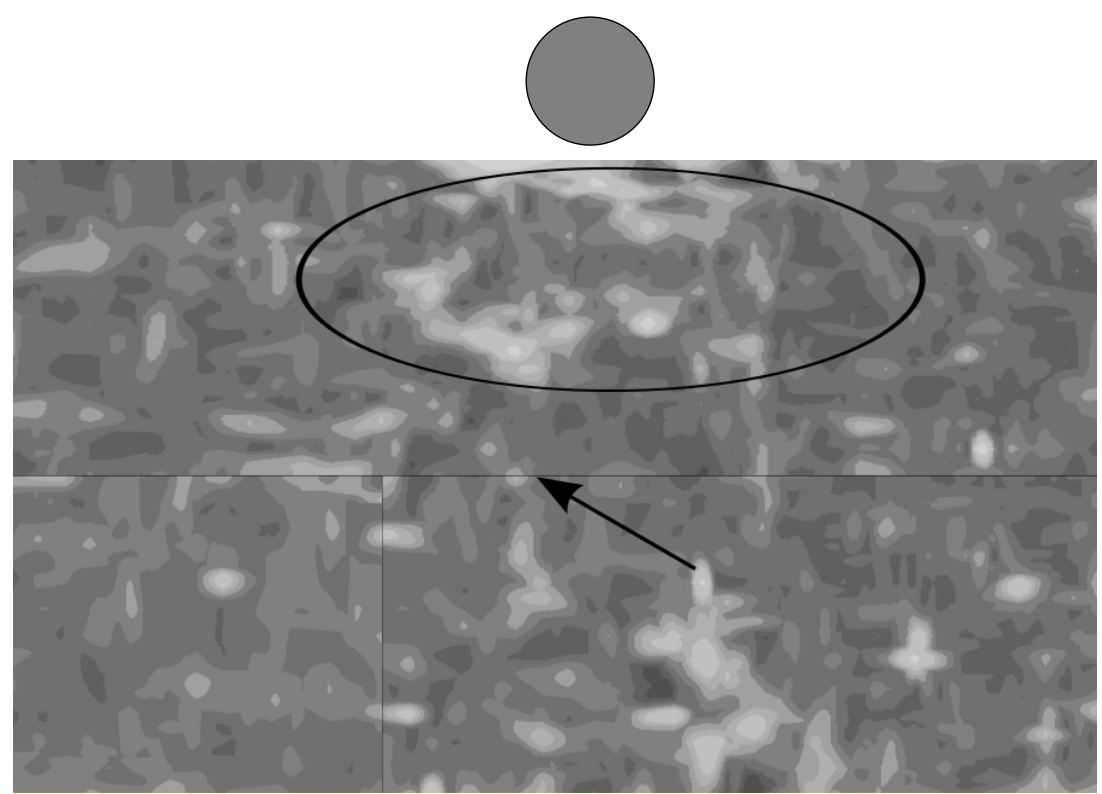

a) Die Parameter $\mathrm{z}$-Wert $=2$; width $=1 ; v=0,12 \mathrm{~m} \mathrm{~ns}^{-1} ; \varepsilon_{r}=6,25$ ergeben einen Tiefenbereich von $9,0 \mathrm{~cm}-15,0 \mathrm{~cm}$.

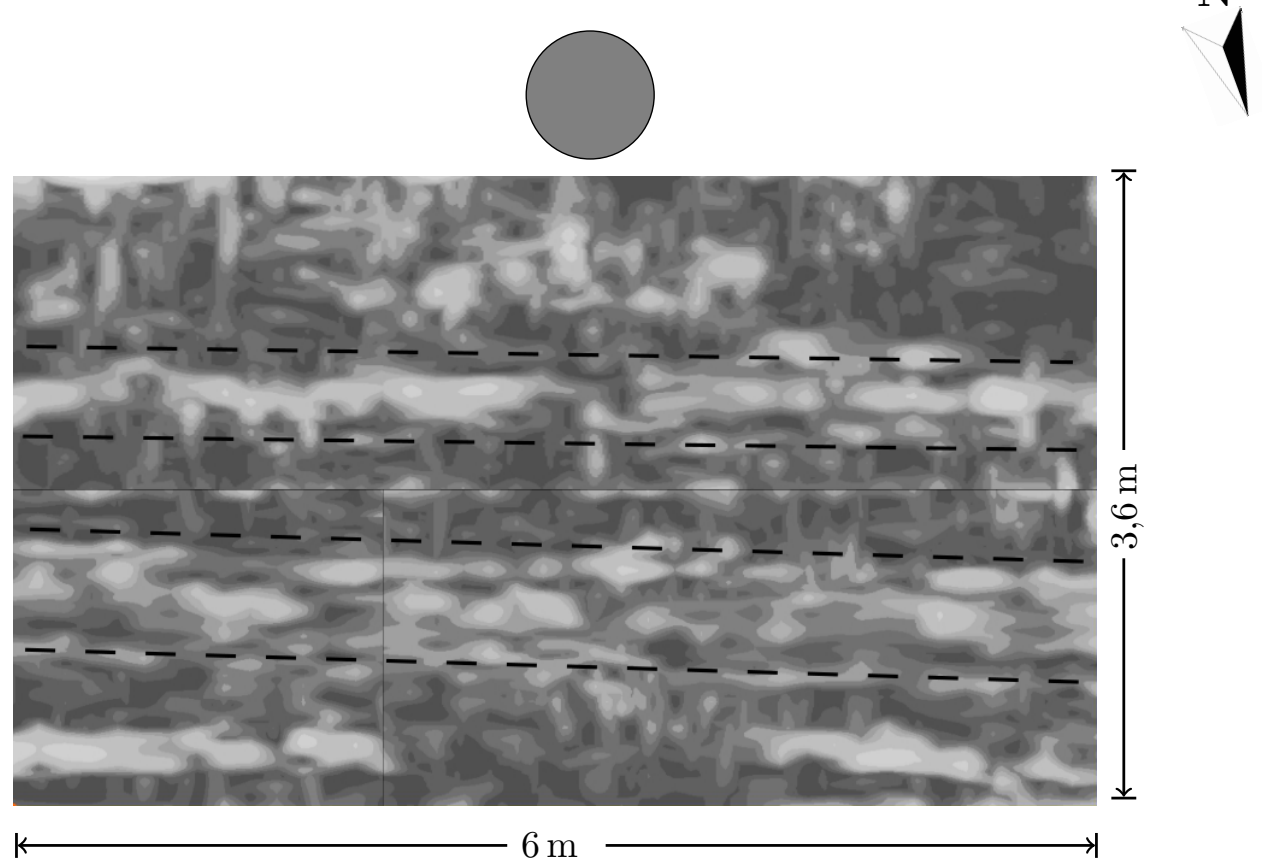

b) Die Parameter z-Wert $=14$; width $=4 ; v=0,12 \mathrm{mns}^{-1} ; \varepsilon_{r}=6,25$ ergeben einen Tiefenbereich von $45,0 \mathrm{~cm}-60,0 \mathrm{~cm}$.

Abbildung 4.41: Ergebnis der Georadaruntersuchung auf der Messfäche Kleine Kastanie in Form von ausgewählten Zeitscheiben. 
Für die Widerstandstomogramme gilt wie bei der benachbarten Messfläche, dass in Baumnähe eine hochohmige Anomalie bis in die letzten erfassten Tiefenstufen vorhanden ist. Während in den obersten Tomogrammen die hochohmigen Areale als einzelne Flecken in einer niederohmigen Matrix vorliegen (Abbildung 4.42a), weist das Tomogramm in 0,2 $\mathrm{m}$ Tiefe fast durchgehend erhöhte Widerstände auf (Abbildung 4.42b). Ab 0,4 m Tiefe liegt ein hochohmiger Streifen am oberen Bildrand im Bereich der Kanalmauer vor (Abbildung 4.42c). Die auf dieser Messfläche verzeichneten absoluten Widerstände liegen zwischen sehr niedrigen Werten und $600 \Omega \mathrm{m}$.

Abbildung 4.43 zeigt das Ergebnis einer Geoelektrikmessung mit einer langen Auslage im Bereich der beiden untersuchten Kastanien als uferparallelen Vertikalschnitt. Während die Sondierungstiefe bei der Detailmessung an der Großen Kastanie etwa 3,0 m betrug, wird durch die längere Auslage eine Sondierungstiefe von etwa 4,0 m erreicht. Erst durch diese vergrößerte Sondierungstiefe wird ersichtlich, dass ab einer Tiefe von etwa 3,0 m eine starke Leitfähigkeitszunahme am Corneliusufer vorliegt. Diese Leitfähigkeitszunahme lässt sich durch den Kanalpegel erklären, der hier etwa $2 \mathrm{~m}$ unterhalb des Bodenniveaus im Baumbereich liegt. 


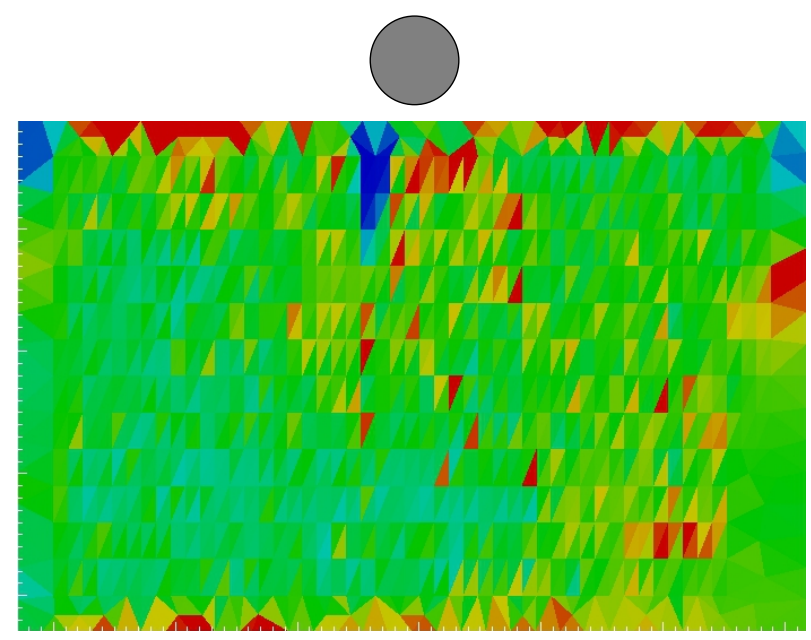

a) Tiefe $0 \mathrm{~m}$
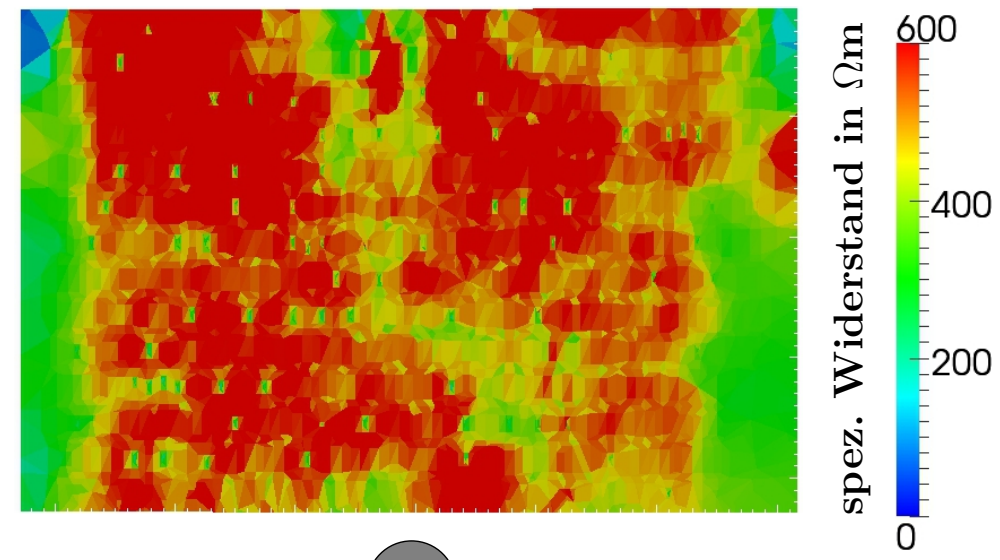

b) Tiefe $0,2 \mathrm{~m}$

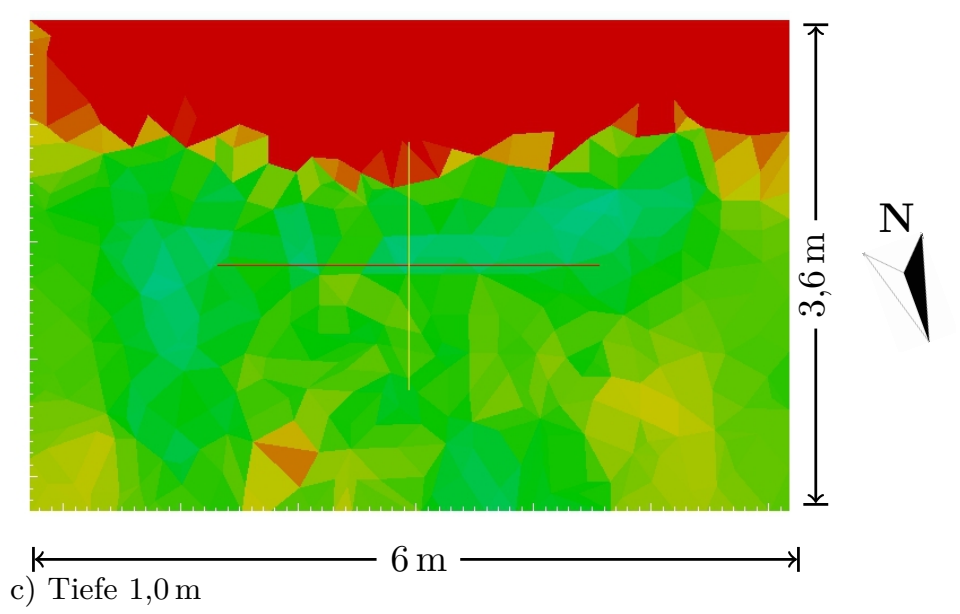

Abbildung 4.42: Ergebnis der Geoelektrikuntersuchung auf der Messfläche Kleine Kastanie in Form von ausgewählten Horizontalschnitten. 


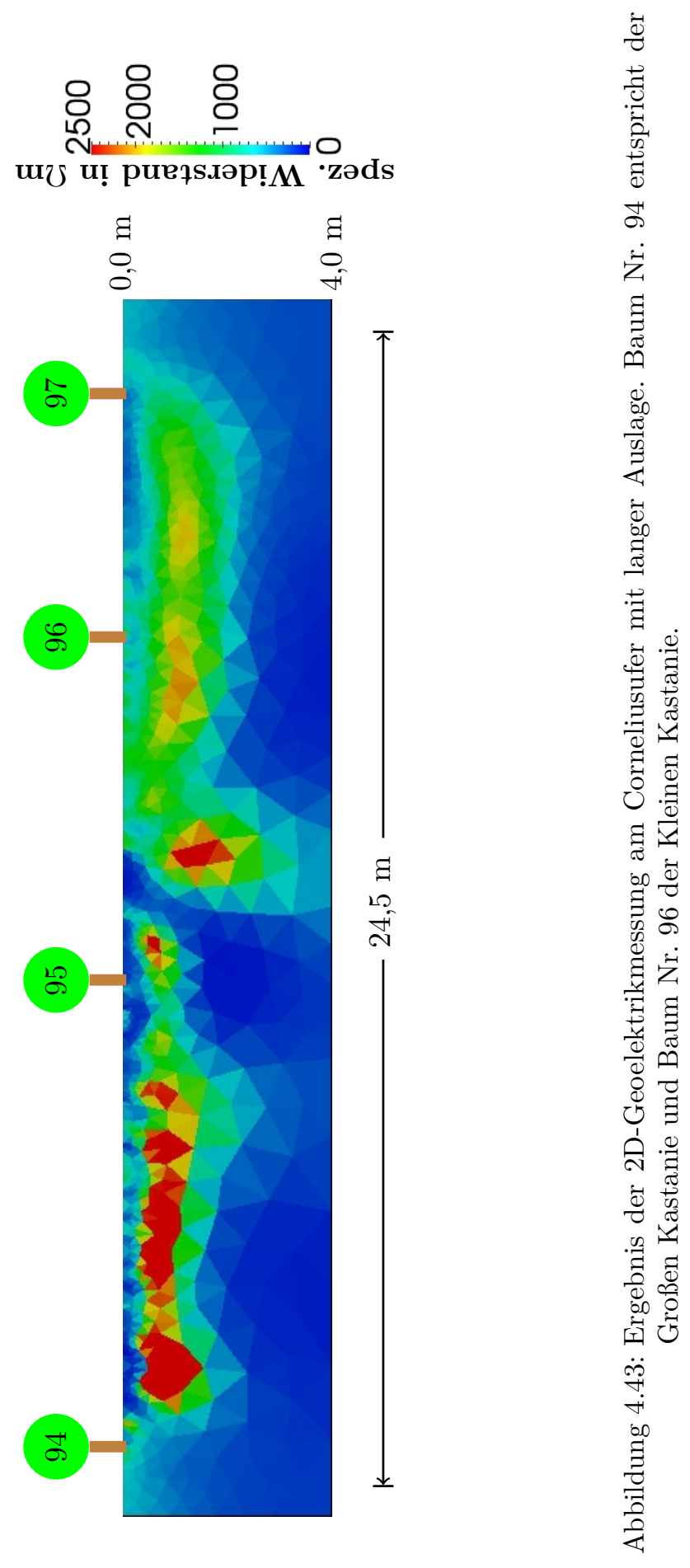


Zusammenfassung der Befunde am Landwehrkanal Auf den Messflächen am Berliner Landwehrkanal fallen die Ergebnisse unterschiedlich aus. Am Einsteinufer sind die Wurzeln der Bäume deutlich und hochauflösend in den Ergebnissen der Radarmessungen abgebildet, während die Geoelektrikmessungen alleine an diesem Standort keine eindeutige Identifikation der Wurzeln ermöglichen.

Am Corneliusufer kann dagegen weder anhand der Radar- noch der Geoelektrikmessungen der Aufbau der Wurzelsysteme ermittelt werden.

An beiden Standorten kann die Tiefenlage des Kanalpegels in den Geoelektrikmessergebnissen als deutliche Leitfähigkeitszunahme gut nachvollzogen werden. 


\subsubsection{Weitere Messflächen}

\subsubsection{Amberbaum Göttingen}

Das Ergebnis der Radaruntersuchung auf der Messfläche Amberbaum (Abbildung 4.44) weicht von den Ergebnissen auf den bisher beschriebenen Messflächen ab.

Im Bereich des Baumes liegt eine flächige Reflexion mit geringer lateraler Ausdehnung vor (Abbildung 4.45a O). In einer Entfernung von etwa $2 \mathrm{~m}$ wird diese von einer linienhaften Struktur eingerahmt (Abbildung $4.45 \mathrm{a} \longrightarrow$ ), von der einzelne lineare Strukturen $4.45 \mathrm{a} \longrightarrow$ ) nach innen und außen abzweigen. Verlauf und Lage dieser Anomalie deuten daraufhin, dass diese Reflexion nicht durch Teile des Wurzelsystems des Amberbaums verursacht wird. Eine sinnvollere Erklärung für diese Reflexion erschließt sich durch die hohe Dichte an Maulwurfshügeln auf der Messfläche (Abbildung 4.44), die im Bereich der beschriebenen Anomalie liegen. So sprechen Form und Lage der Anomalie dafür, dass sich hier das die einzelnen Maulwurfshügel verbindende Gangsystem im Radargramm abzeichnet.

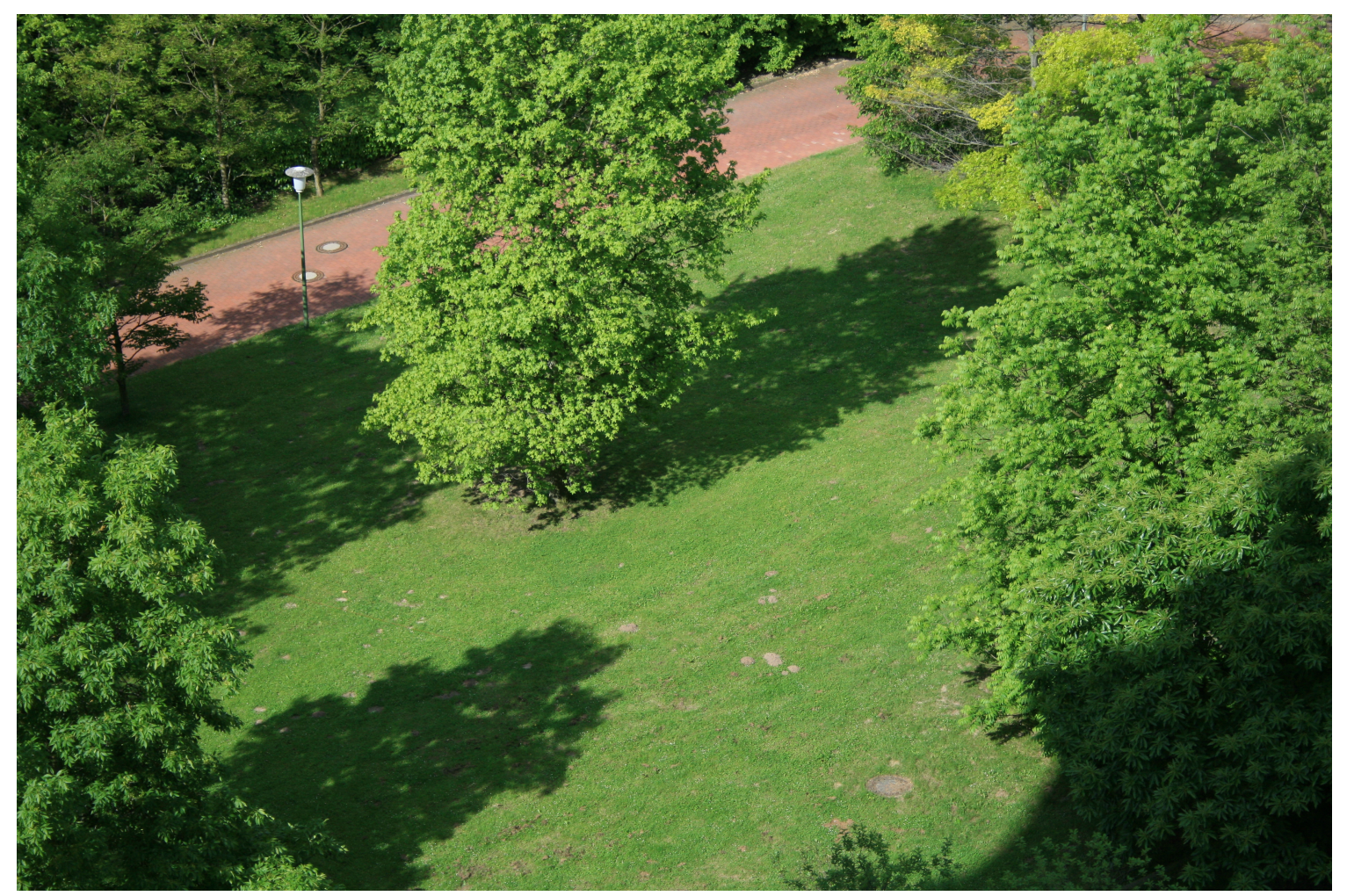

Abbildung 4.44: Foto der Messfläche Amberbaum. 


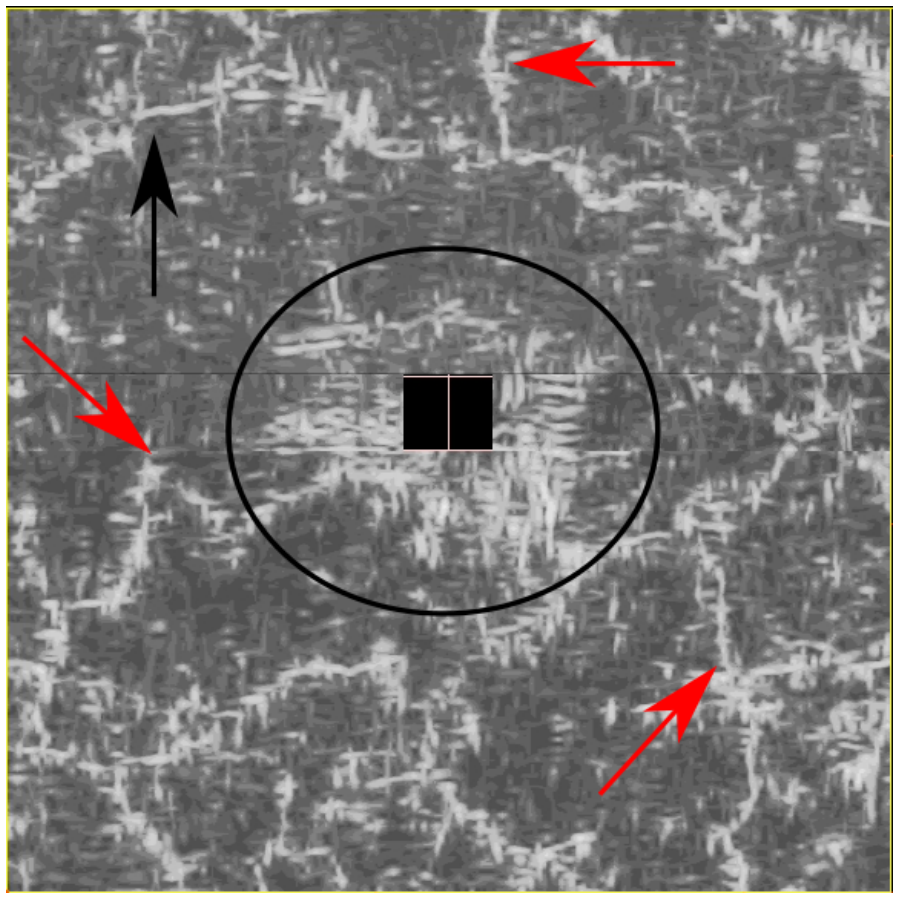

a) Die Parameter z-Wert $=2$; width $=2 ; v=0,12 \mathrm{~m} \mathrm{~ns}^{-1} ; \varepsilon_{r}=6,25$ ergeben einen Tiefenbereich von $6,0 \mathrm{~cm}-18,0 \mathrm{~cm}$.

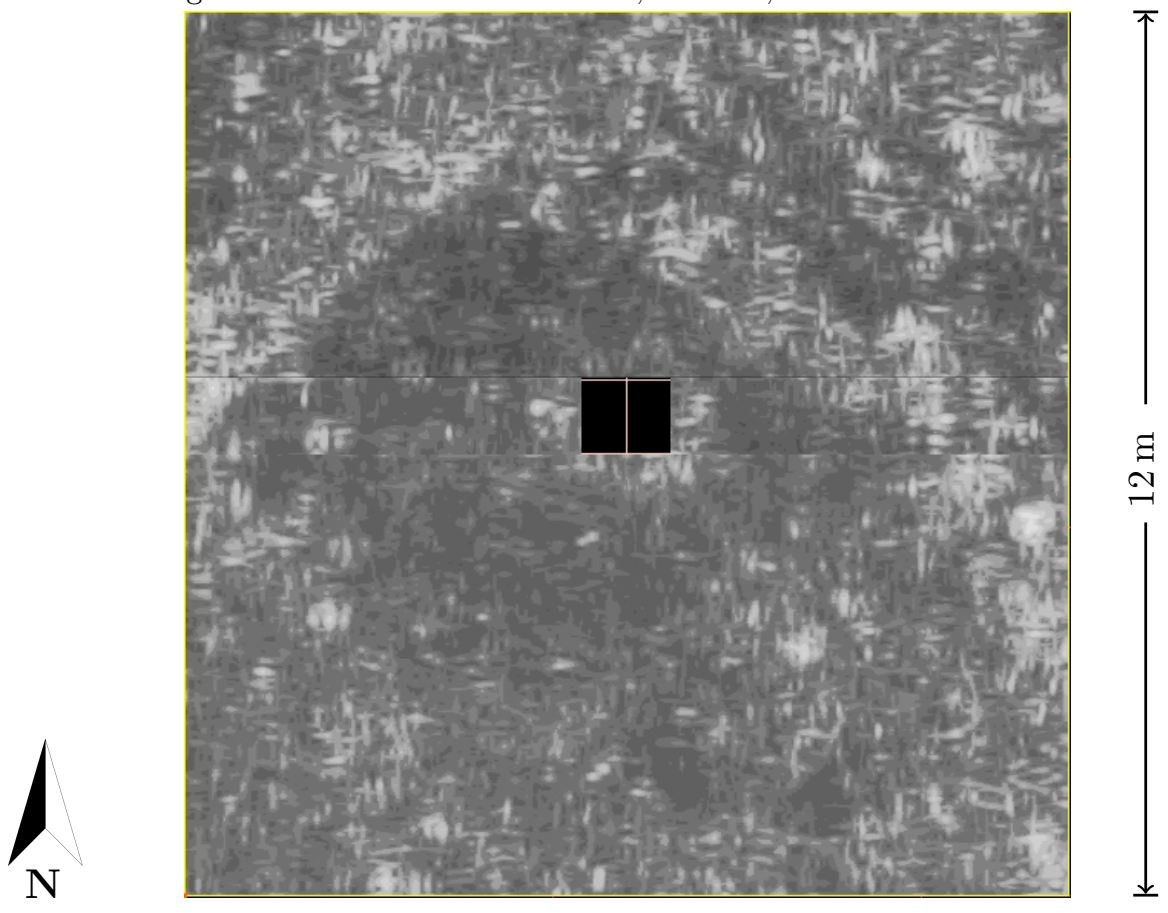

b) Die Parameter z-Wert $=10$; width $=4 ; v=0,085 \mathrm{~m} \mathrm{~ns}^{-1} ; \varepsilon_{r}=12,46$ ergeben einen Tiefenbereich von $34,0 \mathrm{~cm}-51,0 \mathrm{~cm}$.

Abbildung 4.45: Ergebnis der Georadaruntersuchung auf der Messfläche Amberbaum in Form von ausgewählten Zeitscheiben. 


\subsubsection{Kastanienreihe Goldschmidtstraße}

Die Ergebnisdarstellung der Radaruntersuchung an den Kastanien in der Goldschmidtstraße erfolgt in Form eines Vergleichs der Messergebnisse im Umfeld von zwei der drei an diesem Standort untersuchten Bäume.

Die Radargramme der beiden in Abbildung 4.46 dargestellten Messflächen unterscheiden sich dabei trotz der räumlichen Nähe der Untersuchungsobjekte in Bezug auf die enthaltenen Informationen zu den Wurzelsystemen. So lassen sich in der linken Bildhälfte nicht so deutliche Informationen auf Baumwurzeln wie in der rechten finden.

Auch die Laufzeiten, zu denen die Wurzeln in den Radargrammen auftreten, unterscheiden sich. Während die deutlichsten Indikationen bei Baum 1 zu einer Laufzeit von 2 ns gefunden werden (Abbildung 4.46a $\bigcirc$ ), sind in den Zeitscheiben von Baum 2 (Abbildung 4.46d ' - - ') sowohl zu einer Laufzeit von $2 \mathrm{~ns}$ als auch bei einer Laufzeit von 6 ns deutliche Wurzeln zu erkennen. Die Identifikationsmerkmale sind dabei in beiden Fällen die unmittelbare Lage der Anomalien vor dem Baum und die zunehmende Verzweigung nach außen (z.B. Abbildung 4.46 d $\longrightarrow$ ). Zu den Ergebnissen im linken Radargramm ist anzumerken, dass hier die Möglichkeit besteht, dass die Fortsetzungen der im oberflächennahen Radargramm abgebildeten Wurzelanläufe im tiefer liegenden Radargramm dargestellt ist, und somit eine abtauchende Wurzel erfasst worden ist.

Die Wurzeln von Baum 2 haben im Radarergebnis eine größere laterale Ausdehnung und einen stärkeren Kontrast zum Hintergrund als die des Nachbarbaums. Die Identifikation der zusammenhängenden Wurzeln wird in beiden Fällen durch zahlreiche isolierte Reflexionen erschwert.

In einer größeren Tiefe als $6 \mathrm{~ns}$ fehlen bei beiden Beispielen die Indikationen für Wurzeln. In Abbildung 4.46c ist die Zuleitung zu einem von der Oberfläche erkennbaren Hydranten zu sehen (Abbildung 4.46; zwischen _..- ). 
Baum 1

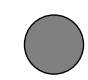

Baum 2

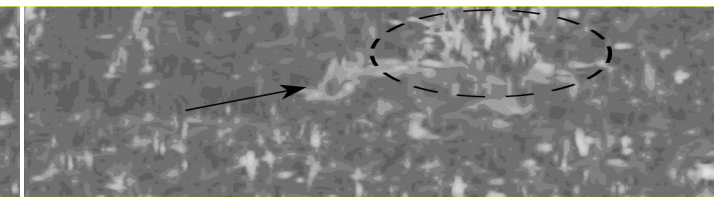

d) $\mathrm{z}$-Wert $=2$; width $=1$ a) z-Wert $=2 ;$ width $=1$
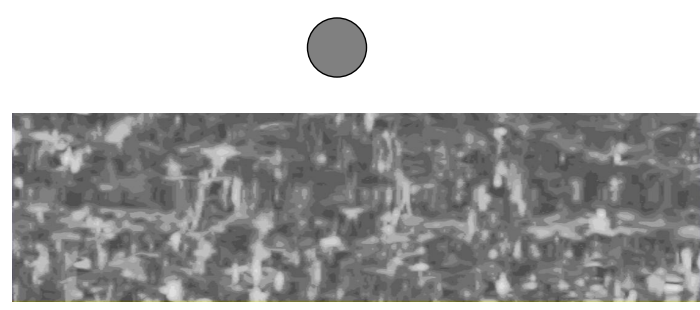

b) z-Wert $=6$; width $=1$

e) $\mathrm{z}$-Wert $=6 ;$ width $=1$

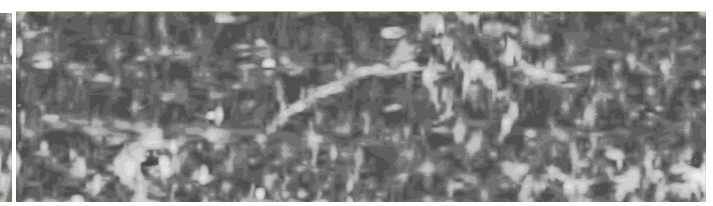

$\mathrm{N}$

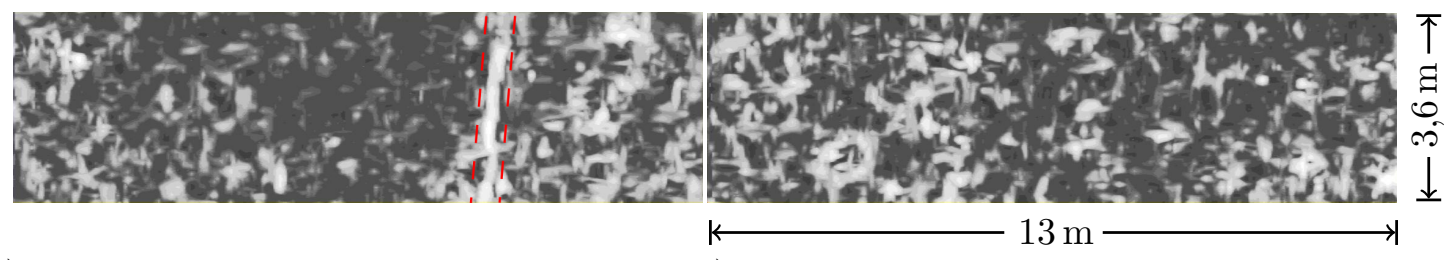

c) z-Wert $=10 ;$ width $=1$

f) $\mathrm{z}$-Wert $=10$; width $=1$

Abbildung 4.46: Ergebnisse der Georadaruntersuchungen auf der Messfläche Kastanien Goldschmidtstraße in Form ausgewählter Zeitscheiben. Tiefenumrechnung:

Die Parameter z-Wert $=2$; width $=1 ; v=0,11 \mathrm{mns}^{-1} ; \varepsilon_{r}=7,44$ ergeben einen Tiefenbereich von $8,25 \mathrm{~cm}-13,25 \mathrm{~cm}$.

Die Parameter z-Wert $=6$; width $=1 ; v=0,095 \mathrm{~m} \mathrm{~ns}^{-1} ; \varepsilon_{r}=7,44$ ergeben einen Tiefenbereich von $26,13 \mathrm{~cm}-30,86 \mathrm{~cm}$.

Die Parameter z-Wert $=6$; width $=1 ; v=0,065 \mathrm{mns}^{-1} ; \varepsilon_{r}=21,30$ ergeben einen Tiefenbereich von $30,86 \mathrm{~cm}-34,13 \mathrm{~cm}$. 


\subsubsection{Kastanie Nimwegen}

In den auf der Messfläche Nimwegen erzielten Ergebnissen sind keine Indikationen dafür auszumachen, dass hier das Wurzelwerk des untersuchten Baumes abgebildet ist.

Die Ergebnisse lassen sich so zusammenfassen, dass eine flächige Reflexion (Abbildung 4.47a (-)') in unmittelbarer Nähe zum Baum, sowie eine in Richtung der Straße verlaufende Reflexion (Abbildung 4.47a zwischen _..--) in einem Abstand von etwa 2,5 m zum Baum vorliegen. In Bereichen, in denen lineare Strukturen wurzelähnlich aus der flächigen Anomalie (z.B. Abbildung $4.47 \mathrm{a} \longrightarrow$ ) herausstreichen, konnten bei Freilegungen (vgl. Kapitel 4.2.6.1) keine Wurzeln gefunden werden. Für die weitere Interpretation der Messergebnisse an diesem Standort wird auf Kapitel 4.2.6.1 verwiesen. 

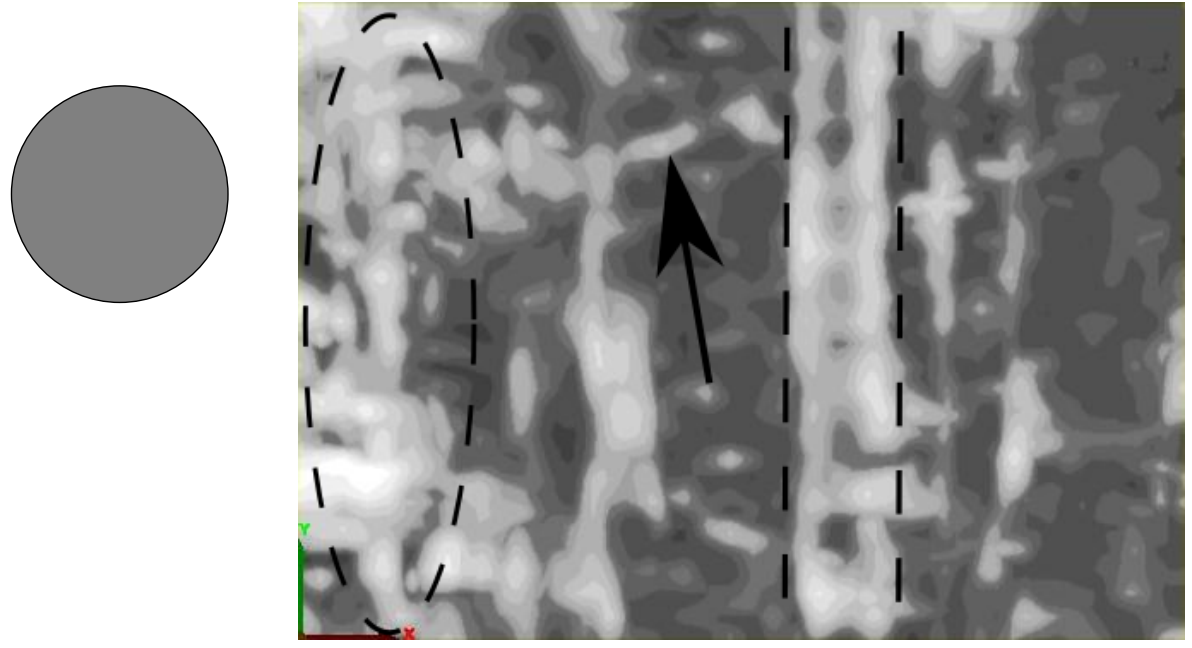

a) Die Parameter z-Wert $=2$; width $=1 ; v=0,12 \mathrm{mns}^{-1} ; \varepsilon_{r}=6,25$ ergeben einen Tiefenbereich von $9,0 \mathrm{~cm}-15,0 \mathrm{~cm}$.
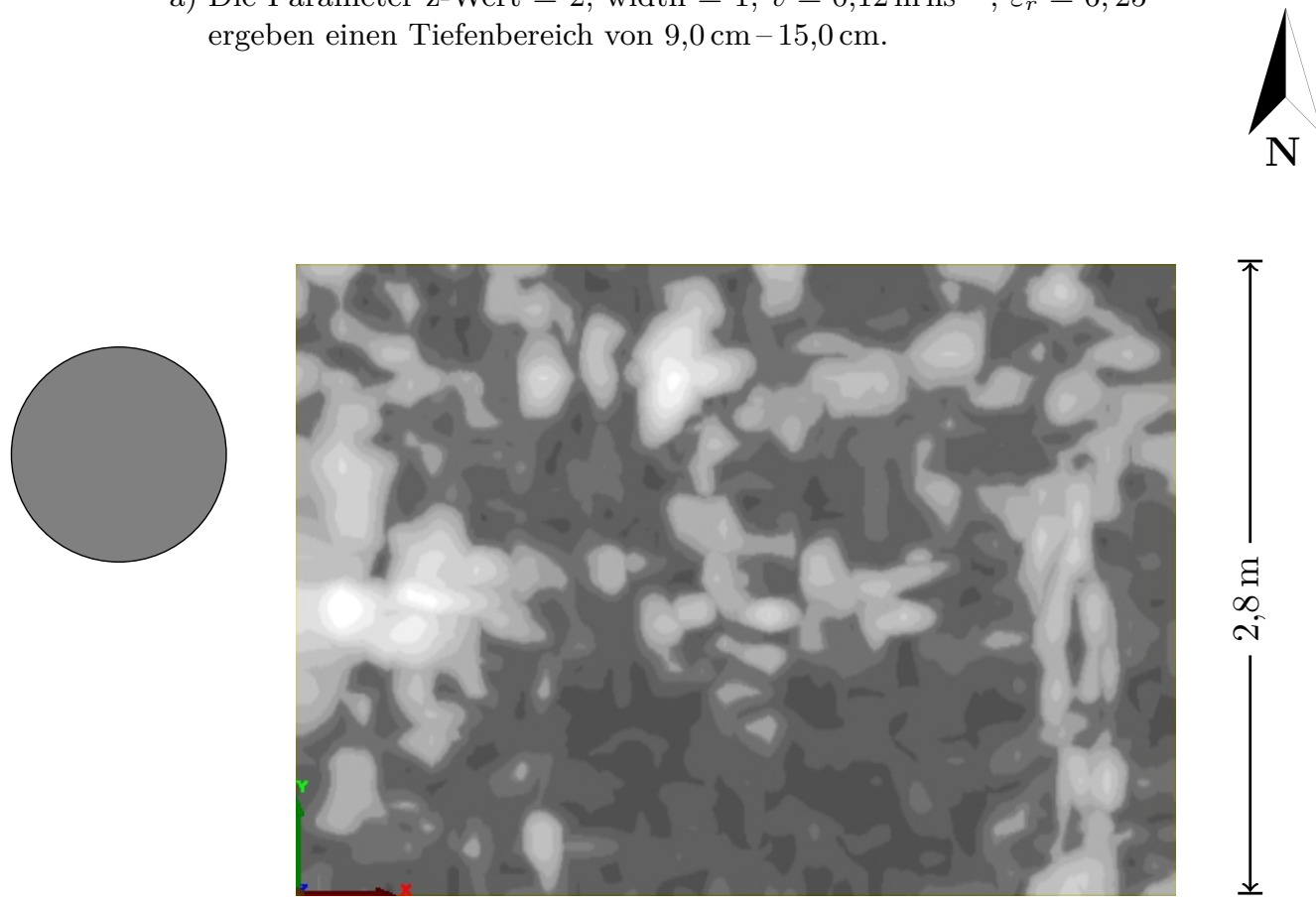

$3,9 \mathrm{~m}$

b) Die Parameter z-Wert $=10$; width $=4 ; v=0,12 \mathrm{~m} \mathrm{~ns}^{-1} ; \varepsilon_{r}=6,25$ ergeben einen Tiefenbereich von $48,0 \mathrm{~cm}-72,0 \mathrm{~cm}$.

Abbildung 4.47: Ergebnis der Georadaruntersuchung auf der Messfläche Kastanie Nimwegen in Form von ausgewählten Zeitscheiben. 


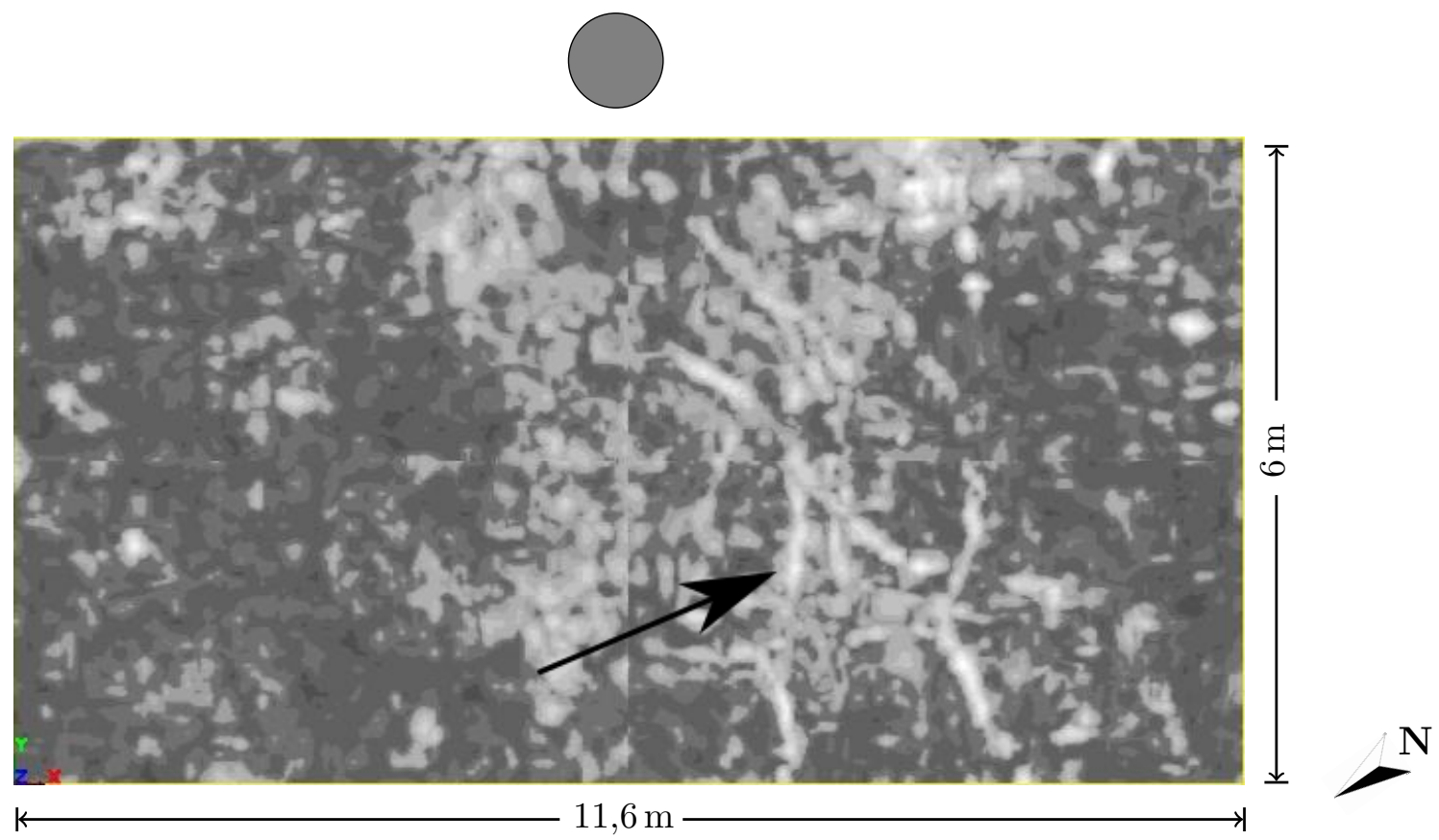

Abbildung 4.48: Ergebnis der Georadaruntersuchung auf der Messfläche Kastanie Fulda in Form der Zeitscheibe. Die Parameter z-Wert $=8$; width $=3 ; v=0,06 \mathrm{mns}^{-1} ; \varepsilon_{r}=25$ ergeben einen Tiefenbereich von $19,5 \mathrm{~cm}-28,5 \mathrm{~cm}$.

\subsubsection{Kastanie Fulda}

Die Ergebnisse der Messfläche Kastanie Fulda (Abbildung 4.48) weisen eine auffällige Zweiteilung auf. Diese ist dadurch gekennzeichnet, dass in der rechten Bildhälfte stellenweise deutliche Wurzeln zu erkennen sind (z.B. Abbildung $4.48 \longrightarrow$ ), während das Radargramm die linke Seite der Messfläche als wurzelfrei ausweist.

Diese Ergebnisse konnten durch Wurzelfreilegungen (vgl. Kapitel 4.2.6.1) bestätigt werden. Für die weitere Interpretation wird auf die in Kapitel 4.2.6.1 beschriebenen Aufgrabungen verwiesen.

Zusammenfassung der Befunde auf den weiteren Messflächen Die Radaruntersuchungen auf den weiteren Messflächen bestätigen die Ergebnisse auf den anderen Untersuchungsflächen, wobei auf der Messfläche Kastanie Fulda die Wurzeln der Kastanie am deutlichsten dargestellt werden können. Auf der Untersuchungsfläche Kastanien Goldschmidtstraße Göttingen gelingt es ebenfalls die Wurzeln der Bäume zu visualisieren, wobei hier kleinräumige Differenzen in den Radargrammen der beiden dargestellten Bäume vorhanden sind. Auf der Messfläche Kastanie Nimwegen gelingt es nicht, Informationen über die Wurzelverläufe des untersuchten Baumes aus den Messdaten der Radaruntersuchung abzuleiten. 
Das Untersuchungsbeispiel Amberbaum zeigt, dass neben anthropogenen Objekten wie Leitungen auch andere Untergrundbestandteile außer Wurzeln, hier vermutlich Maulwurfsgänge, geortet werden können. 


\section{Diskussion}

\subsection{D-Georadarmessungen in den Versuchsbecken}

Die in den Versuchsbecken erzielten Ergebnisse bestätigen die Untersuchungen anderer Autoren, die gezeigt haben, dass sich Wurzeln bei zweidimensionalen Georadarmessungen als Hyperbeln abzeichnen. Dabei konnten mit der eingesetzten $400 \mathrm{MHz}$-Antenne Wurzeln bis zu einem Minimaldurchmesser von $3 \mathrm{~cm}$ in einer Bodentiefe von $40 \mathrm{~cm}$ detektiert werden. Barton und Montagu (2004) konnten mit einer $500 \mathrm{MHz}$-Antenne, die eine entsprechend bessere Auflösung hat, einen Minimaldurchmesser von $1 \mathrm{~cm}$ detektieren. Während Lorra und Kathage (2004) $(400 \mathrm{MHz})$ und Leucci (2011) (500 MHz), die mit ähnlichen Frequenzen gearbeitet haben, keine Angaben zu den detektierten Minimaldurchmessern machen, konnten Hruska et al. (1999) (450 MHz) Wurzeln mit Durchmessern von $3 \mathrm{~cm}-4 \mathrm{~cm}$ unterscheiden.

Bei den Ergebnissen in den Versuchsbecken ist auffällig, dass bei Wurzeln, die einen bestimmten Durchmesser überschreiten, zwei Hyperbeln im Radargramm auftreten. Dieser Effekt ist auch in den von Barton und Montagu (2004) und Hirano et al. (2008) präsentierten Ergebnissen zu beobachten, wird von den jeweiligen Autoren aber nicht erwähnt. In der Studie von Barton und Montagu (2004) ist das Auftreten einer zweiten Hyperbel nicht das Resultat eines größeren Wurzeldurchmessers, sondern einer höherfrequenten Messantenne mit einer entsprechend besseren vertikalen Auflösung. Bei Hirano et al. (2008) wird dieser Effekt dagegen wie in der vorliegenden Arbeit durch die Variation der Wurzeldurchmesser erreicht. Bei der von Hirano et al. (2008) eingesetzten $900 \mathrm{MHz}$-Antenne tritt eine zweite Hyperbel bei Wurzeldurchmessern über $50 \mathrm{~mm}$ auf.

Die Autorengruppen Barton und Montagu (2004) und Hirano et al. (2008) bestimmen jeweils hohe Korrelationen zwischen den Wurzeldurchmessern und den von ihnen angewendeten Konzepten der zero-crossings (siehe hierzu Barton und Montagu 2004, S. 1328) und amplitude-areas (siehe hierzu Hirano et al. 2008, S. 3). Dagegen legen die in den Ergebnissen der vorliegenden Arbeit und den Ergebnissen der genannten Autoren identifizierten übereinanderliegenden Hyperbeln den Schluss nahe, Wurzeldurchmesser zukünftig anhand des Konzepts der umlaufenden Welle zu berechnen, da sich hier die Identifikation von Wurzeloberkante und Wurzelunterkante physikalisch begründen lässt und die zu berechnenden Wurzeldurchmesser sich in Abhängigkeit der Zwei-Wege-Laufzeiten berechnen lassen, wie es auch bei anderen Anwendungen des Georadars geschieht. Den Ergebnissen zufolge lassen sich mit der eingesetzten $400 \mathrm{MHz}-$ Antenne aufgrund ihrer vertikalen Auflösung Wurzeldurchmesser über $10 \mathrm{~cm}$ anhand der umlaufenden Welle berechnen.

Die Grenze des vertikalen Auflösungsvermögens zeigte sich auch bei den Versuchen, übereinander liegende Wurzeln zu unterscheiden. In den entsprechenden Versuchsanordnungen liegt eine Anomalie mit einer größeren vertikalen Ausdehnung vor, bei der allerdings keine Differenzierung 
der einzelnen Wurzeln möglich ist. Hirano et al. (2008) unterscheiden Wurzeln ab einem vertikalen Abstand von $20 \mathrm{~cm}$ bei Messungen mit einer $900 \mathrm{MHz}$-Antenne. Allerdings werden in der zitierten Studie keine Rohdaten präsentiert. So wird nicht deutlich, ob sich im Praxisfall zwischen Messungen unterscheiden ließe, bei denen zwei Hyperbeln bei einem größeren Objekt durch eine umlaufende Welle hervorgerufen werden, und Messungen, bei denen die beiden Hyperbeln jeweils zu einem einzelnen Objekt gehören.

Die Wuchsrichtungen von Wurzeln konnten aufgrund der begrenzten Größe der Versuchsbecken nur ansatzweise untersucht werden. An einer Versuchsanordnung (Becken 2 Position 10) konnte gezeigt werden, dass Wurzeln mit vertikalem Verlauf nicht detektiert werden können. Dies wurde bereits von Lorra und Kathage (2004) angemerkt. Butnor et al. (2006) haben zur Detektion und Visualisierung von einzelnen Pfahlwurzeln Bohrloch-Georadarmessungen erfolgreich eingesetzt. Eine Alternative könnten Georadar-Tomographie-Messungen sein, wie sie von Hagrey (2007) zur Erstellung von Geschwindigkeitsprofilen durchgeführt worden sind.

Eine Wurzel mit diagonaler Wuchsrichtung wurde in Becken 2 an Position 9 simuliert. Hier konnte das Objekt mit schwächerer Amplitude als die anderen Wurzeln in Becken 2 detektiert werden. Die Detektion diagonal in die Tiefe laufender Wurzeln wird im Zusammenhang mit der Visualisierung von dreidimensionalen Messungen weiter diskutiert.

Während sich andere Studien darauf konzentriert haben, wie sich das Aufösungsvermögen des Bodenradars bei der Wurzelortung entsprechend dem Konzept der Fresnelschen Zone mit zunehmender Bodentiefe verändert, zeigen die Untersuchungen in den Lysimeterbecken, dass hier Interferenzen zwischen der Bodenreflexion und der georteten Wurzel vorliegen, wenn die Vertikaldistanz zwischen Wurzel und Bodenoberfläche zu klein ist. Das legt den Schluss nahe, dass für eine sichere Identifikation einer Wurzeloberkante zur weiterführenden quantitativen Analyse (z.B. Wurzeldurchmesserbestimmung) ein ausreichender vertikaler Abstand zur Bodenoberfläche bestehen muss. In den Lysimeterbecken wurde mit der $400 \mathrm{MHz}$-Antenne eine Laufzeit von etwa 5,8 ns für die Bodenreflexion ermittelt. Alteköster (2004) gibt bei Messungen mit einer baugleichen Antenne auf trockenem Waldboden einen Wert von 4,58 ns für die Bodenreflexion und 5,08 ns bei trockenem Waldboden mit Laubauflage an. Werte, wie sie in den Versuchsbecken ermittelt worden sind, können daher auch nur als Richtwerte verstanden werden. Unter anderen Bodenverhältnissen wird die Bodenreflexion von den ermittelten Werten abweichen.

Bei den Untersuchungen zur horizontalen Aufösung des zur Verfügung stehenden Radarequipments konnten die Ergebnisse von Hirano et al. (2008) bestätigt werden. Die Autoren konnten mit der von ihnen verwendeten $900 \mathrm{MHz}$-Antenne Wurzeln mit einem horizontalen Abstand von $20 \mathrm{~cm}$ unterscheiden. Mit der im Rahmen der vorliegenden Arbeit eingesetzten $400 \mathrm{MHz}$-Antenne konnten Wurzeln bis zu einem horizontalen Abstand von $25 \mathrm{~cm}$ unterschieden werden. Bei den Messungen zur Unterscheidung von Baumwurzeln und anderen Materialien hat sich gezeigt, dass die Reflexionsformen von Wurzeln und Objekten wie Kabeln, Rohren und Leitungen im 2D-Radargramm ähnlich sind. Die Amplituden sind bei metallischen Objekten allerdings deutlich höher. Zur Unterscheidung beziehungsweise Identifikation der Objekte im Praxisfall erscheint eine dreidimensionale Messung zur Ermittlung des Objektverlaufs einfacher. Bei der Interpretation flächiger Messungen können, wenn vorhanden, zusätzlich oberirdische Objekte wie Hydranten, 
Straßenlaternen, Gullies oder Bäume zur Interpretation herangezogen werden.

\subsection{D-Georadarmessungen zu grundlegenden Fragestellungen}

Bei diesem Untersuchungsschwerpunkt konnte ein geeignetes Mess- und Visualisierungskonzept ermittelt werden. Zusätzlich konnte gezeigt werden unter welchen Bedingungen die Messungen verifiziert werden können und wie der Zusammenhang zwischen den Messergebnissen und den tatsächlichen Untergrundbedingungen ist.

Bei der Antennenpolarisation hat sich ein xy-Raster mit Beibehaltung der relativen Antennenanordnung (orthogonal) zur jeweiligen Fahrspur als zur Wurzelvisualisierung geeignet erwiesen. Es ist bekannt, dass - wie bei der Ortung anderer Objekte - auch bei Baumwurzeln der Erfolg der Untersuchung von der Lage der Polarisationsrichtung der eingesetzten Antennen zu den Zielobjekten abhängig ist. Hier konnte gezeigt werden, dass durch das in dieser Arbeit gewählte Untersuchungsschema sowohl Objekte, die primär parallel zur x-Achse verlaufen, als auch Objekte, die primär parallel zur y-Achse verlaufen, detektiert werden können. Auch Objekte, die unter anderen Winkeln verlaufen, konnten durch den kombinierten Einsatz beider Aufnahmerichtungen visualisiert werden. Dabei war die Aufnahme in Form von linearen Transekten durch das Auswerteprogramm RADAN 6.6 vorgegeben.

Andere Softwarepakete (z.B. das Programm gpr-slice), die in Verbindung mit geeigneten Positionierungssystemen (GPS oder lokale Totalstationen) die Aufnahme von beliebigen Transektformen gestatten, standen nicht zur Verfügung. Andere, teilweise eher kommerziell ausgerichtete Systeme zur Wurzeldetektion (www.treeradar.com) nutzen circumpolar um den Baum angeordnete Transekte, da hierdurch die Wurzeln immer annähernd orthogonal überfahren werden. Die von Butnor et al. (2012, S. 231 ff.) präsentierten Ergebnisse erwecken nicht den Anschein, inhaltlich der Aufnahme in Rasterform überlegen zu sein, da hier nur stark abstrahierte Objekte dargestellt werden. Vorteile hat diese Methode sicherlich bei der in die Messungen investierten Zeit, da das exakte Abspannen eines engmaschigen Rasters und die Erfassung der Messspuren bei der in der vorliegenden Arbeit angewendeten Methode mit hoher Wahrscheinlichkeit deutlich länger dauert als die kreisförmige Umfahrung eines Baumes (vgl. Kapitel 5.4.

Den Ergebnissen dieser Arbeit zufolge erweist sich die Rasterdichte als der wesentliche Einflussfaktor auf die erfolgreiche Detektion von Wurzeln mittels Bodenradar. So konnten im Rahmen dieser Arbeit Wurzelverläufe in einem Detailgrad visualisiert werden, wie er in anderen Publikationen zu diesem Thema nicht vorgefunden wird. Zenone et al. (2008) zeigen Übereinstimmungen zwischen den Ergebnissen ihrer Radarmessungen und Wurzelfreilegungen. Die GPR-Ergebnisse wirken dabei allerdings durch den Rasterabstand von $25 \mathrm{~cm}$ treppenartig und nicht kontinuierlich. Auch in den Ergebnissen von Lorra und Kathage (2004) (Rasterabstand $50 \mathrm{~cm}$ ) sind Anomalien im Bereich des Baumes zu erkennen, aus denen allerdings keine detaillierten Wurzelverläufe abgeleitet werden können. Leucci (2011) hat mit einer $500 \mathrm{MHz}$-Antenne Wurzelsysteme von Eukalyptusbäumen untersucht, wobei die Wurzeln bei einem Rasterabstand von $50 \mathrm{~cm}$ ebenfalls als durchgehende Fläche dargestellt werden. Kathage et al. (2011) geben für die Unterscheidung von Leitungen und Wurzeln einen Profilabstand von $1 \mathrm{~m}$ als geeignet an. Der vorliegenden 
Arbeit folgend ist ein solcher Rasterabstand für eine Unterscheidung solcher Objekte bei einer flächenhaften Kartierung nicht geeignet. Die Ergebnisse dieser Arbeit lassen den Schluss zu, dass ein Rasterabstand von $15 \mathrm{~cm}$ geeignet ist, Wurzeln kontinuierlich zu visualisieren und dabei die unvorhersehbaren Richtungswechsel von Baumwurzeln ausreichend nachzuvollziehen. Den Ergebnissen liegen dabei bis auf eine Ausnahme Messungen mit einer $400 \mathrm{MHz}$-Antenne zugrunde. Die zusätzlich erfolgte Messung mit einer $900 \mathrm{MHz}$-Antenne weist aber daraufhin, dass dieser Rasterabstand auch bei höheren Frequenzen zu guten Visualisierungsergebnissen führt.

Bei der Untersuchung der Reproduzierbarkeit konnten die Wurzeln auf der Messfläche Schwarzkiefer wiederholt in gleicher Form visualisiert werden, wobei unter die entsprechenden Messungen auch der genannte Einsatz der $900 \mathrm{MHz}$-Antenne fällt. Durch eine Kreuzkorrelation konnte gezeigt werden, dass ein deutlicher statistischer Zusammenhang zwischen den Messergebnissen auf der Messfläche Schwarzkiefer besteht. Der Korrelationskoeffizient von $\mathrm{R}=0,47$ beim Vergleich aller Bildelemente erhöht sich auf $\mathrm{R}=0,7$, wenn jeweils der Durchschnitt von 15 Pixeln verglichen wird. Die Abweichungen im Bild lassen sich zum einen durch die unterschiedliche Bodenfeuchtigkeit bei der Datenaufnahme erklären. Zum anderen unterscheiden sich auch die Geräteeinstellungen (gain, Distanzerfassung, etc.) bei allen Messungen minimal. Zusätzlich wirken sich die leicht versetzten Messtaster bei beiden Messungen aus. So konnte gezeigt werden, dass sich die Winkel, unter denen die Messantennen zu den Wurzeln verlaufen (vgl. Antennenpolarisation), auf die aufgezeichneten Amplituden auswirken. Ein solcher Effekt ergibt sich auch bei der Untersuchung der Reproduzierbarkeit durch das bei der zweiten Messung um 2,55 gedrehte Messraster. Entsprechende Wiederholungsmessungen konnten in der Literatur zu diesem Thema nicht gefunden werden.

Alteköster (2004) hat die grundsätzliche Reproduzierbarkeit von Georadarmessungen untersucht. Dabei hat er gezeigt, wie sich kleine Veränderungen an den eingesetzten Verbindungskabeln und an der Kontaktfläche Antenne-Boden auf die Messergebnisse auswirken. Solche Auswirkungen von Ankopplungsveränderungen können auch in den meisten Radargrammen wiedergefunden werden und sind zumeist auf Anhebungen der Antenne bei unruhigem Verlauf der Bodenoberfläche zurückzuführen. Durch die großflächigen 3D-Messungen wirken sich diese lokal begrenzten Störungen aber bei der resultierenden dreidimensionalen Ergebnisdarstellung nur geringfügig aus.

Im Zusammenhang mit der Reproduzierbarkeit muss auch die Möglichkeit erwähnt werden, dass Fremdobjekte in stark frequentierten, öffentlich zugänglichen Grünflächen zwischen den zu vergleichenden Messungen eingebracht werden, welche bei Wiederholungsmessungen mit erfasst werden. Dazu gehört beispielsweise neben anthropogenen Objekten wie Kronkorken, Flaschen, etc. auch direkt vom Baum stammendes Material in Form von Ästen, Zapfen und größeren Früchten wie z.B. Kastanien. Die Gefahr einer Fehlinterpretation wird hier durch die dreidimensionale Datenaufnahme minimiert.

Für die Untersuchung der Filter und Nachbearbeitung standen die im Programm RADAN 6.6 implementierten Filter zur Verfügung. Hier ist die Subtraktion der Laufzeit bis zur Bodenreflexion essentiell, um eine realistische Darstellung der Ergebnisse zu erreichen, in der die Bodenoberfläche bei $0 \mathrm{~ns}$ bzw. $0 \mathrm{~m}$ einsetzt. Weiterhin hat sich die Migration der Daten wie in vielen anderen Studien als sinnvoll erwiesen, um die erfassten Objekte an ihre realen Ausmaße anzupassen. Um 
so höher die Geschwindigkeit der Wellen im Boden ist, desto breiter fallen dabei die Hyperbeln in den 2D-Radargrammen aus. Da durch die Migration auch schon Interferenzen der Hyperbeläste reduziert werden, erleichtert sie die Identifikation von Objekten in der zweidimensionalen Ergebnisdarstellung. Diese wird auch durch den Background-Removal-Filter verbessert, da Anomalien im Vergleich zum abgeschwächten Hintergrund stärker hervortreten. Im Kontext mit Filtern hat sich gezeigt, dass für eine erfolgreiche dreidimensionale Wurzeldetektion die Qualität der Ausgangsdaten am wichtigsten ist.

Butnor et al. (2012) erwähnen, dass durch den Einsatz von Filtern in städtischen Böden die Identifikation von Wurzeln verbessert wird. Gerade bei diesen Substraten hat sich bei den im Rahmen der vorliegenden Arbeit gezeigten Datensätze aber herausgestellt, dass durch Filtertechniken keine ausreichende Verbesserung bei der Wurzelidentifikation in 3D-Bodenradarmessungen erreicht wird. Der Aussage von Butnor et al. (2012) kann aber insofern zugestimmt werden, dass einzelne Objekte im 2D-Radargramm durch die Filter deutlicher hervorgehoben werden können.

An verschiedenen Beispielen zur Verifikation konnte gezeigt werden, dass die Annahmen, die aus den Radarmessungen über die Wurzelverteilungen getätigt wurden, den tatsächlichen Gegebenheiten im Untergrund entsprechen. Dies war sowohl bei umfangreicheren Aufgrabungen als auch bei gezielten Stichproben der Fall. Hierbei konnten deutlichere Übereinstimmungen als bei vergleichbaren Untersuchungen anderer Autoren festgestellt werden (Zenone et al. 2008). Aufgrund der verhältnismäßig geringen horizontalen und vertikalen Aufösung des zur Verfügung stehenden Messequipments wurde auf umfangreiche quantitative Untersuchungen verzichtet. Auf Untersuchungsflächen, bei denen in den zugehörigen Radargrammen keine deutlichen Wurzeln identifiziert werden konnten, wurden auch bei den Freilegungen vor Ort keine Wurzeln gefunden. Hier wurden auch methodische Grenzen erreicht. Das war bei Substraten mit einem hohen Skelettanteil der Fall, bei denen aufgrund der Untergrundbeschaffenheit keine tiefgründigen Aufgrabungen möglich waren. Dies waren insbesondere die steinigen und stark verdichteten Standorte am Corneliusufer in Berlin und in Nimwegen. Hier konnte nicht geklärt werden, ob der Untergrundaufbau zu komplex ist, um ihn in der notwendigen Detailschärfe aus den Radarmessungen zu rekonstruieren und hier beispielsweise Wurzelverläufe vorliegen, die sich nicht in das typische Muster von sich nach außen verzweigenden linearen Strukturen einordnen lassen.

Bei Butnor et al. (2012) wird ebenfalls erwähnt, dass es Schwierigkeiten bei der Wurzelidentifizierung in Radargrammen und der Visualisierung von Wurzeln bei einem hohen Skelettanteil gibt.

\subsection{D-Georadar- und Geoelektrikmessungen auf ausgewählten Standorten}

In der vorliegende Arbeit wurden erstmals umfangreiche 3D-Messungen zur Wurzeldetektion präsentiert, die einen Vergleich der Ergebnisse an unterschiedlichen Standorten ermöglichen und sich dabei nicht nur auf einen oder wenige Standorte mit sehr guten Visualisierungsergebnissen beschränken. Die Untersuchungen haben gezeigt, dass Baumwurzelverläufe über weite horizontale Distanzen anhand von Georadarmessungen visualisiert werden können. Voraussetzung für eine 
gelungene Darstellung der Wurzelsysteme war dabei, dass sich das jeweilige Wurzelsystem über keine großen Vertikaldistanzen erstreckt, sondern annähernd in einer Ebene verläuft, da sich hierdurch die Wurzeln als zusammenhängende Anomalien in einer Zeitscheibe darstellen lassen.

Bei Roberts et al. (2009) finden sich Hinweise darauf, dass es zur Identifizierung von Zielobjekten notwendig sein kann, entsprechend dünne Zeitscheiben zu analysieren. Die Autoren führen das darauf zurück, dass in dickeren Zeitscheiben schwächer reflektierende Zielobjekte von Störsignalen (noise) und anderen, stärkeren Signalen überlagert werden können. Dies konnte für die Wurzeldetektion bestätigt werden.

Eine weitere Schwierigkeit scheint die Anpassung der Signalverstärkung (gain) zu sein, die vor jeder Messung vorgenommen wird. Diese soll sicherstellen, dass die Daten vollständig aufgezeichnet werden und die Amplituden nicht abgeschnitten werden (kein clipping der aufzuzeichenden Daten stattfindet). Gleichzeitig werden tiefliegende Reflexion entsprechend verstärkt, um die mit zunehmender Laufzeit erfolgende Signalabschwächung auszugleichen. Beim Vorhandensein eines starken Reflektors wie einer Leitung wird die Signalverstärkung dabei sehr niedrig eingestellt, um Signalamplituden, die über den Aufzeichnungsbereich hinausgehen (clipping), zu verhindern. Der niedrige Wert bei der Signalverstärkung führt aber auch dazu, dass die Reflexionen der Wurzeln ebenfalls eine Intensitätsabstufung erfahren, die die Identifikation in Zeitscheiben erschweren kann. Ergebnisse wie die von Hruska et al. (1999), die komplexe Wurzelverläufe in mehreren übereinander liegenden Ebenen zeigen, erscheinen vor diesem Hintergrund unrealistisch.

Bei den unterschiedlichen Standorten konnten in schluffigen und sandigen Böden aussagekräftige Ergebnisse zum Verlauf der Wurzelsysteme erzielt werden. Zweidimensionale Untersuchungen auf vergleichbaren Standorten, die die generelle Eignung dieser Substrate für Radarmessungen zur Wurzelortung zeigen, finden sich bei Butnor et al. (2001).

Die Differenzierung von Wurzeln und anderer Objekte konnte dabei in verschiedenen Fällen durch die Topologie (Lage und Verlauf) der abgebildeten Anomalien erfolgen. In diesem Zusammenhang konnten auch andere Objekte mit einem komplexen Verlauf wie Maulwurfsgänge von den Wurzeln unterschieden werden.

Um an Standorten, an denen keine Aufgrabungen möglich sind, weitere Informationen über den Untergrundaufbau und die dazu gehörenden Wurzelverhältnisse zu erhalten, wurden zusätzlich geoelektrische Messungen durchgeführt. Beim Vergleich mit entsprechenden Literaturangaben zu den spezifischen Widerständen verschiedener Materialien wird ersichtlich, dass sich die Größenordnungen der ermittelten spezifischen Widerstände in erster Linie durch das Substrat an den untersuchten Standorten erklären lassen.

Bei den Messflächen Schwarzkiefer und Buchengruppe wurde die Wurzelmasse ermittelt, um durch statistischen Analysen einen möglichen Zusammenhang zwischen der Wurzelmasse und den Widerstandswerten zu ermitteln, wie es Amato et al. 2008) und Rossi et al. (2010) gelungen ist. Für den Fall der Buchengruppe konnte bei drei der untersuchten Transekte ein Zusammenhang zwischen dem Widerstand und der Wurzelmasse ermittelt werden, der wie bei den genannten Autoren dadurch gekennzeichnet ist, dass hier eine Zunahme der Wurzelmasse mit höheren Widerständen einhergeht. Da bei dem vierten Transekt der Buchengruppe und bei der Schwarzkiefer in drei Fällen ein gegenläufiger Zusammenhang festgestellt worden ist, lässt sich 
für diesen Teil der Untersuchung kein eindeutiges Ergebnis formulieren. Diese Trendumkehr lässt sich auch nicht durch die Verteilung der an den Standorten gemessenen Bodenfeuchte erklären.

Denkbar ist, dass es hier zu Verzerrungen durch die nicht berücksichtigte Bodentemperatur gekommen ist. Die Widerstandswerte wurden bei Amato et al. (2008) und Rossi et al. (2010) jeweils um diesen Faktor korrigiert. Für diese Hypothese spricht, dass bei der insgesamt gleichmäßiger beschatteten Buchengruppe der Zusammenhang zwischen spezifischem Widerstand und Wurzelmasse dem bei den genannten Autorengruppen ermittelten Trend folgt, dass der Widerstand mit Zunahme der Wurzelmasse steigt. Die im Rahmen dieser Untersuchungen gewonnenen Erkenntnisse reichen nicht zur Formulierung allgemeiner Rückschlüsse aus, sondern erfordern weiterführende Messungen (vgl. Kapitel 5.5).

Rossi et al. (2010) merken an, dass bei ihren Messungen im Widerstandsbereich von $17 \Omega \mathrm{m}-$ $28 \Omega \mathrm{m}$ keine detektierbare Wurzelmasse vorhanden war und zwischen $30 \Omega \mathrm{m}-35 \Omega \mathrm{m}$ kein signifikanter Zusammenhang zwischen spezifischem Widerstand und Wurzelmasse bestand. Die Autoren verweisen darauf, dass auch Amato et al. (2008) erst ab Widerstandswerten von $40 \Omega \mathrm{m}$ in der Lage waren, Wurzeln zu detektieren. Rossi et al. (2010) vermuten, dass diese Grenzen je nach Hintergrundwiderstand und Standort variieren können.

Daraus lässt sich schließen, dass die elektrische Widerstandstomographie bei entsprechenden Bodenverhältnissen ein geeignetes Verfahren zur Wurzelmassenbestimmung sein kann. Es gibt aber auch Standorte, auf denen andere Faktoren einen dominierenden Einfluss auf den spezifischen Widerstand ausüben und sich die Wurzelmassenverteilung nicht aus diesem rekonstruieren lässt. Als entsprechende Faktoren werden bei Rossi et al. (2010) neben dem Bodenwassergehalt, der Salzgehalt und die Lagerungsdichte genannt. Für die Rolle der Wurzeln führen Rossi et al. (2010) an, dass bei ihren Messungen mehr als $25 \%$ des Bodenvolumens durchwurzelt waren, was eine mögliche Erklärung für den deutlichen Einfluss der Wurzeln auf den spezifischen Widerstand des Bodens liefert.

Die Ergebnisse der Bodenradaruntersuchungen wurden nicht herangezogen, um analog zu den Arbeiten von Butnor et al. (2003b) Biomassebestimmung durchzuführen. Dies ist darin begründet, dass die Ergebnisse in den Versuchsbecken gezeigt haben, dass sich mit dem eingesetzten Bodenradarequipment Wurzeln ab einem Durchmesser von $4 \mathrm{~cm}$ bestimmen lassen und die Wurzelbeprobungen auf der Schillerwiese (Buchengruppe und Schwarzkiefer) im wesentlichen Feinwurzeln mit deutlich geringeren Durchmessern beinhalten.

Die Überlagerung von Radargrammen und Tomogrammen zeigt stellenweise eine gute Deckungsgleichheit von als Wurzeln identifizierten Anomalien im Radargramm und hochohmigen Bereichen. Dass hier kein vollkommene Deckungsgleichheit vorliegt, lässt sich durch grundsätzliche Unterschiede der eingesetzten Verfahren erklären. So weist das Georadar eine deutlich höhere Auflösung als die Geoelektrik auf. Weiterhin muss berücksichtigt werden, dass die Leitfähigkeit von Objekten richtungsabhängig ist und es sich daher auf die Messergebnisse auswirkt, ob eine Wurzel parallel oder orthogonal zur Elektrodenauslage verläuft. Während dieser Geometrieeinfluss beim Bodenradar durch die Befahrung mit verschiedenen Antennenanordnungen auf allen Messflächen berücksichtigt worden ist, wurden bei der Geoelektrik nur am Landwehrkanal orthogonale Elektrodenauslagen eingesetzt. Bei den übrigen Messflächen wurden parallele Auslagen eingesetzt. 


\subsection{Anwendbarkeit der Messverfahren in der Praxis}

Die Messungen am Landwehrkanal, die Messung in Nimwegen und die Messung in Fulda waren in praktische Fragestellungen eingebunden.

Am Landwehrkanal konnten die Messergebnisse dazu beitragen, die Ausmaße der Wurzelsysteme am Einsteinufer abzuschätzen und erbrachten damit wichtige Informationen für die Planung von Sanierungsmaßnahmen an den maroden Ufermauern des Landwehrkanals. Diese Informationen konnten am Corneliusufer durch den Einsatz des Georadars und der elektrischen Widerstandstomographie nicht erbracht werden. Anzumerken ist, dass auch anhand von Aufgrabungsergebnissen die Morphologie der Wurzeln an diesem Standort nicht geklärt werden konnte.

In Fulda wären die Informationen aus den Radarmessungen dazu geeignet gewesen, die Wurzelverhältnisse am Untersuchungsstandort ausreichend zu beurteilen, um die Lage von Punktfundamenten bei einer Baumaßnahme wurzelschonend durchführen zu können. Die tatsächliche Bauplanung orientierte sich hier an den Ergebnissen der Wurzelsuchgrabung.

In Nimwegen konnte die Durchwurzelung des städtischen Extremstandorts nicht aus den Radarmessungen abgeleitet werden. Allerdings konnte diese auch hier nicht durch die durchgeführte Wurzelfreilegung ausreichend analysiert werden.

Es lässt sich aus den in Kapitel 4 dargelegten Befunden schließen, dass flachstreichende Wurzelsysteme in homogenen Böden mit einer hohen Wahrscheinlichkeit durch den Einsatz des Georadars hochauflösend visualisiert werden können. Schwierigkeiten bei der Interpretation treten dagegen auf, wenn die Morphologie der Wurzeln dazu führt, dass diese sich in den 3DRadargrammen nicht als sich nach außen verzweigende lineare Strukturen abzeichnen, und eine zunehmende Komplexität im Untergrundaufbau vorliegt. Diese führt zu einer wachsenden Unsicherheit in der Interpretation der Messergebnisse, die soweit reichen kann, dass keine sichere Ansprache von Wurzeln in den Messergebnissen mehr möglich ist.

Der Durchmesser der georteten Wurzeln ist sowohl bei der Anwendung der Methoden in der kommerziellen als auch in der wissenschaftlichen Praxis von Interesse. Im Rahmen dieser Arbeit wurde ein im Bereich der Wurzelortung neuer Ansatz der Durchmesserbestimmung diskutiert. Dieser konnte allerdings bisher nur unter kontrollierten Bedingungen bei einer kleinen Stichprobe verifiziert werden. Für den Einsatz in der Praxis müssen hier noch weitere Untersuchungen folgen (vgl. Kapitel 5.5).

Neben der Frage, ob die Messergebnisse zur Klärung bestimmter Sachverhalte beitragen können, spielt bei der Einschätzung der Praxistauglichkeit auch die Praktikabilität der Messmethoden, insbesondere ein vertretbarer zeitlicher und finanzieller Aufwand, eine Rolle. Diesem Aspekt, der bei einer kommerziellen Anwendung berücksichtigt werden muss, wurde im Rahmen der vorliegenden Untersuchung nicht detailliert nachgegangen. 
Tabelle 5.1: Anwendbarkeit geophysikalischer Verfahren zur Wurzeldetektion

\begin{tabular}{|c|c|c|c|}
\hline \multicolumn{2}{|l|}{ Teilaspekt/ Parameter } & \multirow{2}{*}{$\frac{\text { Georadar }}{++++^{a}}$} & \multirow{2}{*}{$\frac{\text { Geoelektrik }}{++++^{b}}$} \\
\hline Standort & Kontrollierte Bedingungen & & \\
\hline & Natürliche Verhältnisse & $++^{a}$ & $++^{b}$ \\
\hline & Annähernd natürliche Verhältnisse & $+^{a}$ & $+^{a}$ \\
\hline & Extremstandorte & $-a$ & $-a$ \\
\hline \multirow[t]{2}{*}{ Untersuchungsziel } & Wurzelkartierung & $+++^{a}$ & $+++^{c}$ \\
\hline & Wurzelmassenbestimmung & $+d$ & $+++^{b, c}$ \\
\hline \multirow[t]{4}{*}{ Bodenbeschaffenheit } & homogen & $+++^{a}$ & $+++^{b, c}$ \\
\hline & leicht inhomogen & $++^{a}$ & $++e$ \\
\hline & geschichtet & $+^{a}$ & $+^{a}$ \\
\hline & stark inhomogen (z.B. Bauschutt) & $--^{a}$ & $-a$ \\
\hline \multirow[t]{3}{*}{ Bodenoberfläche } & versiegelt & $+^{a}$ & n.a. \\
\hline & grasbewachsen & $+++^{a}$ & $+++^{a}$ \\
\hline & uneben & $-a$ & $+^{a}$ \\
\hline \multirow[t]{3}{*}{ Wurzelsystem } & flachstreichend & $+++^{a}$ & $++^{a}$ \\
\hline & tief & $-a$ & $+^{a}$ \\
\hline & typische Wurzelverläufe & $+++^{a}$ & $+^{a}$ \\
\hline \multirow[t]{2}{*}{ Wurzeldurchmesser } & Feinwurzeln & $-{ }^{a}$ & $++^{b, c}$ \\
\hline & Skelettwurzeln & $+++^{a}$ & $++^{b, c}$ \\
\hline
\end{tabular}

Legende: +++ sehr gut; ++ gut; + geeignet; - schlecht geeignet; - - - vollkommen ungeeignet; n.a. nicht anwendbar

${ }^{a}$ Eigene Arbeit

${ }^{b}$ Amato et al. 2008

${ }^{c}$ Rossi et al. (2010)

${ }_{\text {z.B. Dannoura et al. } 2008}$

${ }^{e}$ Hagrey und Meissner (2004) 

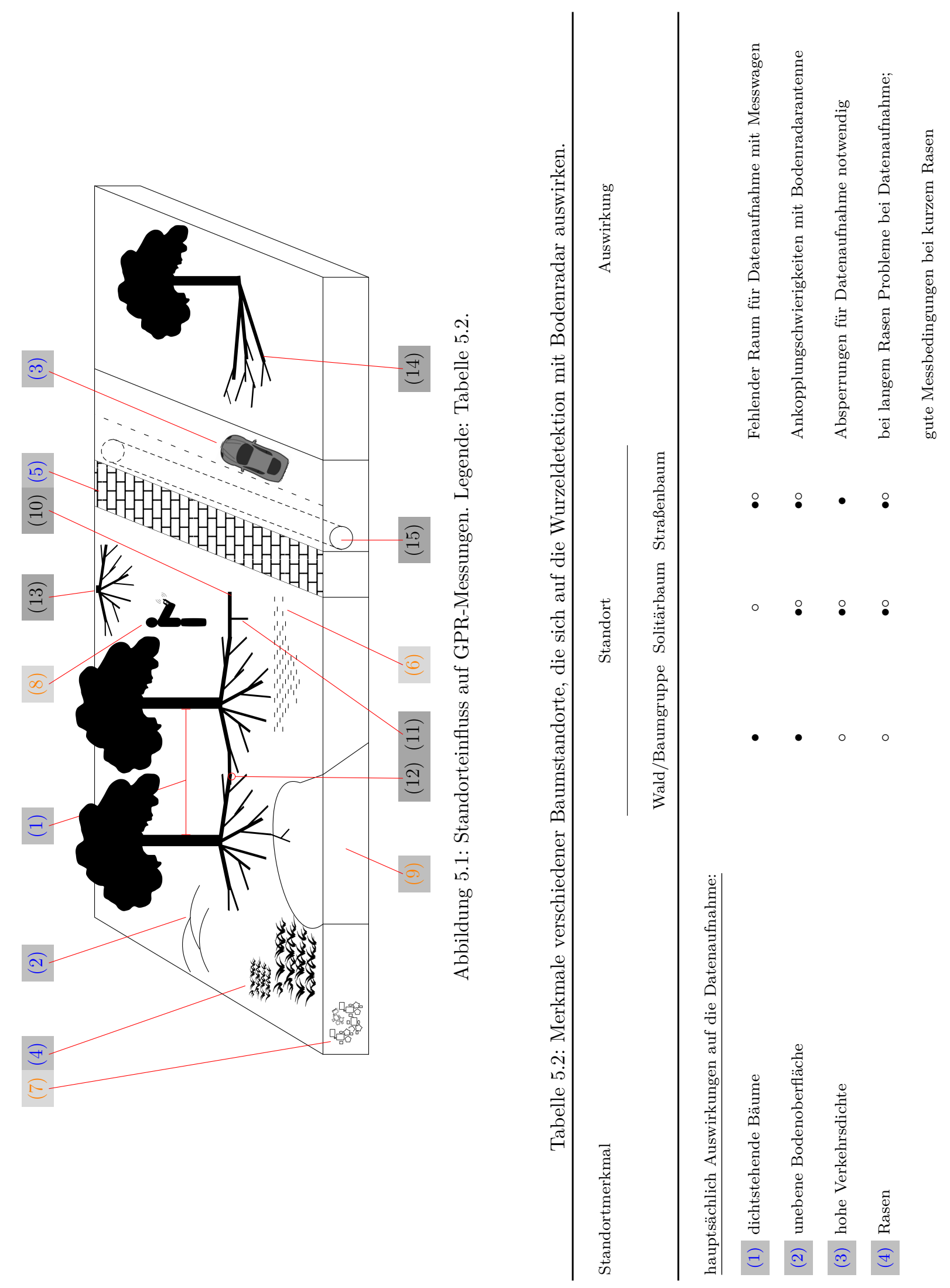


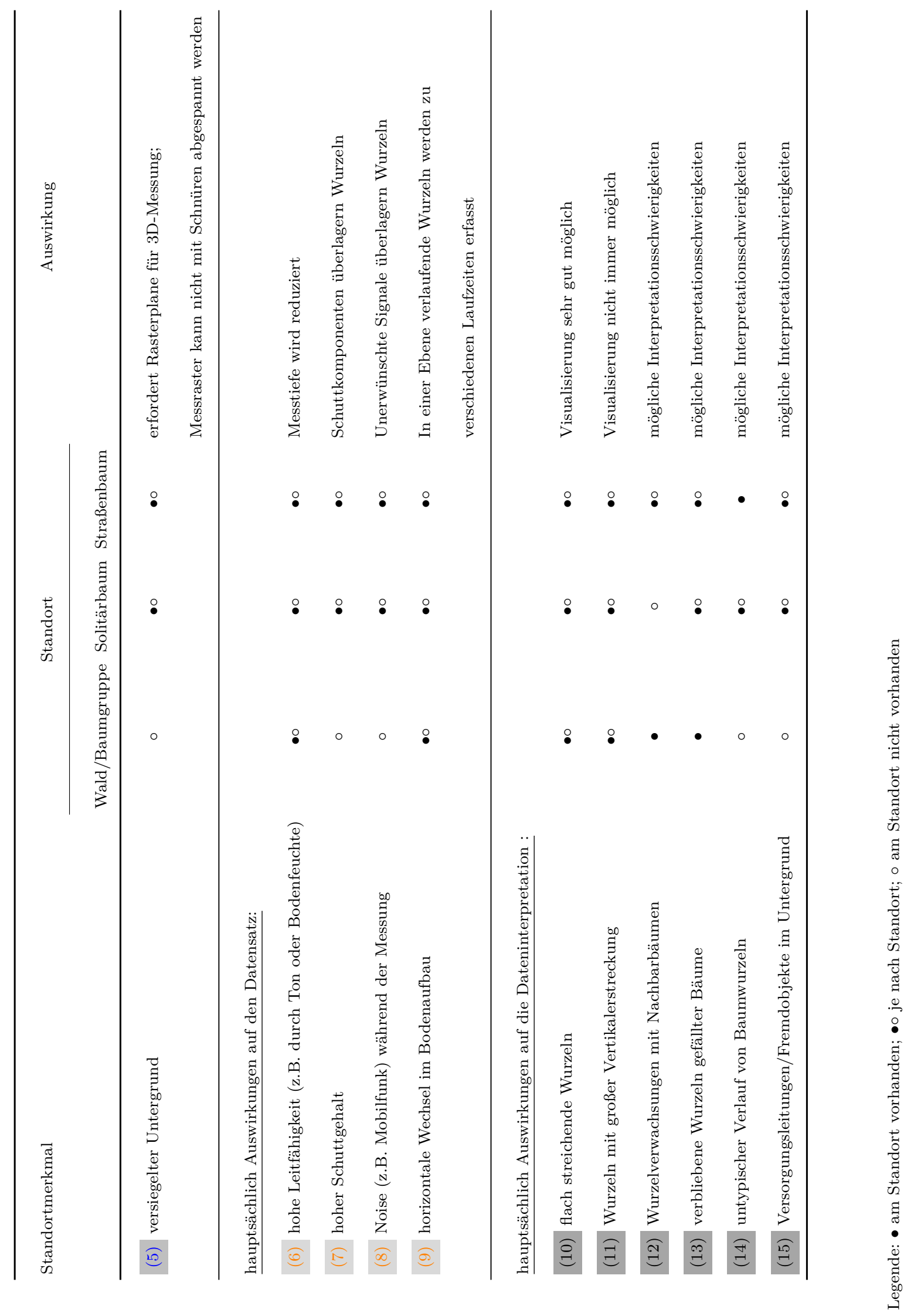


Anzumerken ist hierbei aber, dass eine Messung an einem Baum, in dem präsentierten Detailgrad (Größe der Messfläche: $12 \mathrm{~m} \times 12 \mathrm{~m}$; Rasterabstand: 0,15 m) je nach Beschaffenheit der Messfläche (Hindernisse, Hangneigung, etc.) mindestens einen halben Tag beansprucht. Die Nachbearbeitung der Messergebnisse bis zur Ergebnisdarstellung kann ebenso lange dauern. Dieser hohe Zeitbedarf liefert sicherlich eine Erklärung dafür, warum kommerzielle Anbieter zugunsten schnellerer Messungen auf eine hohe Detailgenauigkeit verzichten, da sie ihrem Kunden nur so ein wirtschaftlich vertretbares zeitliches Voranschreiten der Datenaufnahme anbieten können. Je nach Untergrundbeschaffenheit kann eine Wurzelfreilegung auch sehr zeitintensiv sein, hat aber den Vorteil, dass sie die gewünschten Informationen in jedem Fall liefert. Letztere ist aber mit dem Nachteil verbunden, nicht zerstörungsfrei zu arbeiten.

Die Anwendbarkeit der Verfahren in der Praxis kann daher nicht eindeutig bestätigt werden. Der Erfolg einer geophysikalischen Messung lässt sich abhängig vom Standort erst bei der Sichtung der Messergebnisse beurteilen. Eine grobe Einschätzung des Erfolges kann allerdings durch eine Voruntersuchung des Standorts erfolgen. Die wichtigsten Einflussfaktoren hierbei sind in Tabelle 5.1 zusammengefasst. In dieser Tabelle wird verglichen, wie erfolgversprechend die Einsatzmöglichkeiten von Georadar und Geoelektrik an verschiedenen Standorten und für verschiedene Fragestellungen sind.

In Abbildung 5.1 und Tabelle 5.2 werden darüber hinaus die Auswirkungen von unterschiedlichen Standortmerkmalen auf Georadarmessungen zur Wurzeldetektion illustriert. Dabei wird anhand von den drei exemplarischen Standortklassen Wald/Baumgruppe, Solitärbaum und Straßenbaum abgeschätzt, wie wahrscheinlich das Auftreten eines bestimmten Merkmals an einem Standort ist. Deutlich wird, dass die meisten genannten Standortmerkmale für die einzelnen Standorte unterschiedlich ausfallen können. Dabei ergibt sich zusätzlich für jeden Standort eine Vielzahl möglicher Merkmalkombinationen. Daran wird ersichtlich, dass es nicht möglich ist, aus den Ergebnissen der vorliegenden Arbeit darauf zu schließen, dass die GPR-Wurzeldetektion an bestimmten Standorten per se funktioniert. Stattdessen müssen verschiedene Merkmale in einer Kombination vorliegen, die Folgendes ermöglicht:

- eine praktikable Datenaufnahme

- die Aufnahme eines Datensatzes mit einem aussagekräftigen Signal:Noise-Verhältnis

- eine Interpretation, die mit ausreichender Wahrscheinlichkeit, die Identifikation von Wurzelverläufen ermöglicht

Grundsätzlich stellen die beiden untersuchten Messverfahren eine gute Ergänzung zu den etablierten destruktiven Wurzeluntersuchungen dar. In bestimmten Fällen, wie einem homogenen Standort mit einem flachstreichenden Wurzelsystem kann das Georadar dabei Ergebnisse liefern, anhand derer auch schon alleinstehend die Morphologie eines Wurzelsystems beurteilt werden kann.

Bei der Interpretation der Messergebnisse muss aber immer berücksichtigt werden, dass lediglich physikalische Eigenschaften des Untergrunds kartiert werden, anhand derer sich im Idealfall die 
Wurzelsysteme rekonstruieren lassen und nicht die Wurzeln selbst direkt detektiert werden (vgl. Kapitel 2.3.1).

\subsection{Ausblick und weiterer Forschungsbedarf}

Auf den in der vorliegenden Arbeit vorgestellten Erkenntnissen aufbauend, sollten zwei Bereiche im Forschungsfeld Wurzeldetektion mit Georadar weiter untersucht werden. Dies sind die städtischen Extremstandorte und die in die Tiefe führenden Wurzelstränge, die bei der Anwendung des vorgestellten Mess- und Visualisierungsschemas Probleme bereiten.

Im Fall eines hohen Schuttgehalts könnten höher auflösende Antennen (z.B. $900 \mathrm{MHz}$ ) in Kombination mit einem dichteren Messraster (z.B. 2,5 cm) zu besseren Ergebnissen führen. Dabei ist zu beachten, dass sich bei einem engeren Messraster Ungenauigkeiten in der Datenaufnahme entprechend stärker auswirken. Diese könnten durch den Einsatz von Totalstationen bei der Positionierung des Messgeräts minimiert werden.

Bei der Visualisierung von Wurzeln mit einer größeren Vertikalausdehnung sollten Untersuchungen mit einer weiterführenden Visualisierungssoftware unternommen werden. Beispielsweise bietet das Programm gpr-slice die Möglichkeit einer overlay-analysis genannten Bearbeitungsoption. Durch diese können die Horizontalschnitte einzelner Tiefenstufen einzeln bearbeitet werden, um eine tiefenabhängige Herausarbeitung einzelner Anomalien zu erreichen. In einer anschließenden Überlagerung (overlay) der bearbeiteten Horizontalschnitte könnten dann sich fortsetzende Reflexionen mit unterschiedlichen Amplituden fortlaufend visualisiert werden. Die Software stand für die Erstellung der vorliegenden Arbeit nicht zur Verfügung. Der Entwickler des Programms, Dean Goodman, hat den auf der Messfläche Schwarzkiefer aufgenommenen Datensatz mit der genannten Software ausgewertet (im Rahmen der Tagung „Rundtischgespräch Georadar 2011“ der Deutschen Geophysikalischen Gesellschaft am 11.10.2011). Teile dieser Ergebnisse sind als Beispiel der Darstellungsoptionen von gpr-slice in Abbildung 5.2 dargestellt. Daneben gibt es verschiedene Modellierungsprogramme (u.a. in gpr-slice verfügbar) mit denen die genauen Reflexionsvorgänge beispielsweise auch zweidimensional nachvollzogen werden sollten, um den Einfluss von grobkörnigem Substrat und die beobachteten Effekte beim Vorliegen größerer Wurzeldurchmesser (umlaufende Welle) besser beurteilen zu können. Dadurch könnte auch ein physikalisch begründbares Modell zur Bestimmung des Wurzeldurchmessers etabliert werden.

Im Bereich der Datenaufnahme sollten Messungen mittels Radar- Tomographie oder Bohrlochgeoradar weiterverfolgt werden, um mehr Informationen über Wurzelsysteme mit größerer Vertikalerstreckung zu erhalten. Dabei sollten Störeffekte, die auf grobkörniges Substrat zurückzuführen sind, durch homogene Bodenverhältnisse oder kontrollierte Bedingungen ausgeschlossen werden. 


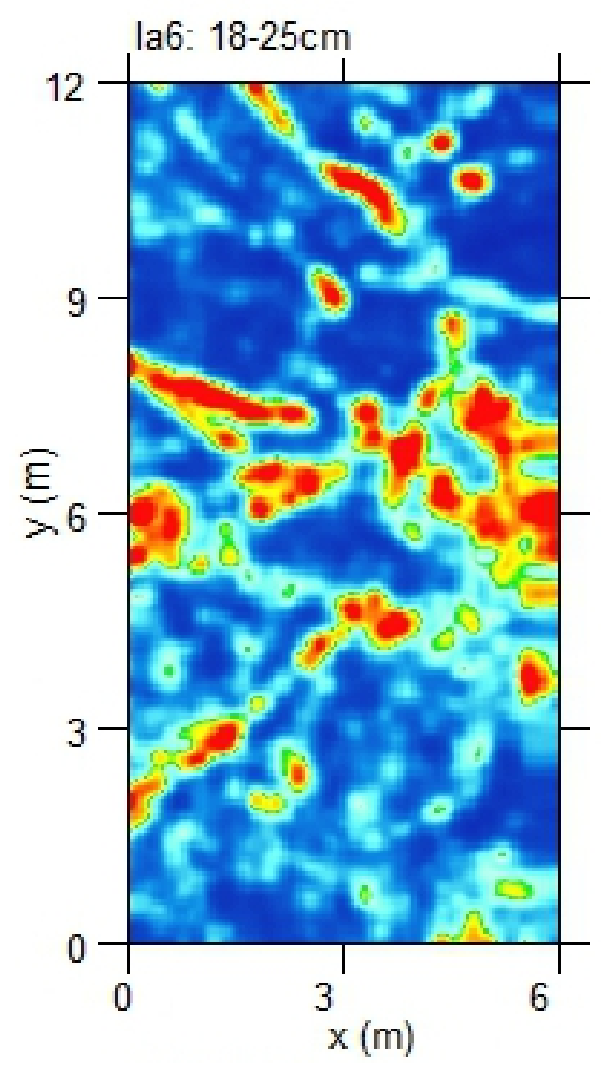

a) Overlay-analysis

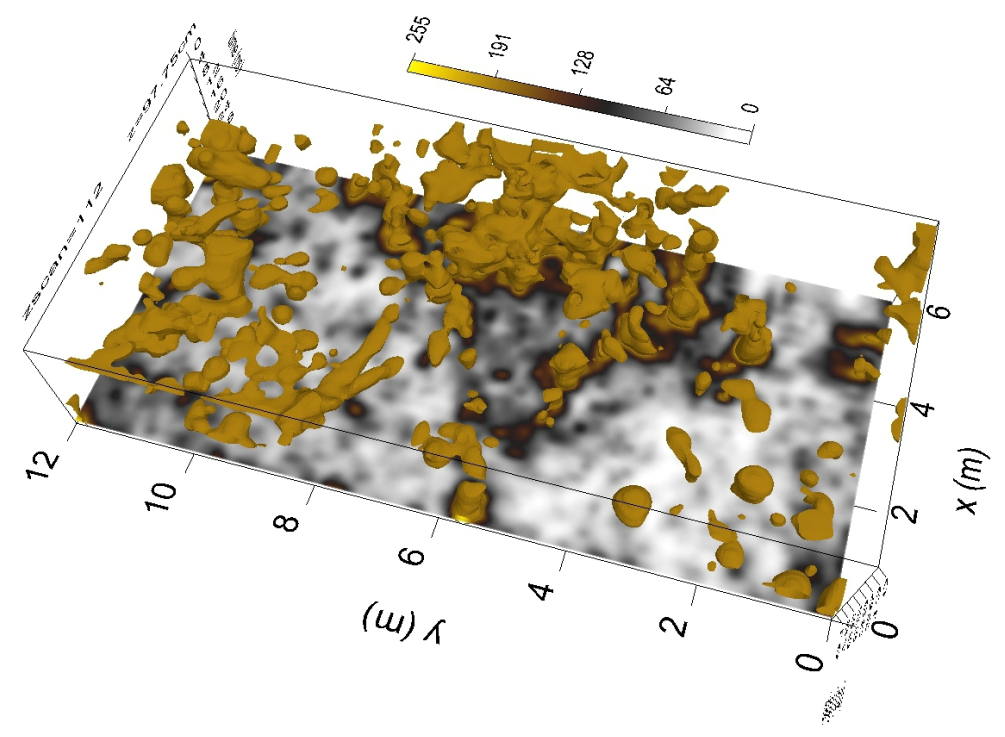

b) ISO-surfaces

Abbildung 5.2: Beispielhafte Visualisierung einer Georadarmessung mit gpr-slice (erstellt von Dean Goodman 07.10.11). 


\section{Zusammenfassung}

Es war das Ziel der Arbeit, die Möglichkeiten und Grenzen des Einsatzes zweier geophysikalischer Verfahren, Georadar und Geoelektrik, für die Ortung von Baumwurzeln im städtischen Raum zu untersuchen und wenn möglich Kriterien anzugeben, anhand derer bestimmt werden kann, ob ein Einsatz dieser Verfahren für den genannten Zweck erfolgversprechend ist. Aufgrund der höheren Auflösung lag der Fokus der Untersuchungen dabei auf dem Georadar.

Vorbereitend zu den eigentlichen Messungen wurden mit dem Georadar Voruntersuchungen von eigens angelegten Versuchsanordnungen durchgeführt. Des Weiteren fanden Untersuchungen statt, die darauf abzielten, ein geeignetes Messschema für die eigentlichen Vergleichsuntersuchungen auf verschiedenen städtischen Standorten zu ermitteln. Im Rahmen dieser Untersuchungen konnte der Einfluss verschiedener Faktoren auf die Ergebnisse der Radaruntersuchungen gezeigt werden. Außerdem wurde ein Auswerteschema festgelegt, um die Messungen auf den weiteren Messflächen vergleichen zu können. Dazu gehörte auch die Wahl einer geeigneten Darstellungsform.

Die kontrollierten Versuchsflächen miteinbezogen wurden drei Arten von Standorten untersucht, kontrollierte Bedingungen, annähernd unverändert und städtische Extremstandorte.

In Kombination mit den Ergebnissen der Voruntersuchungen konnte gezeigt werden, dass sowohl unter kontrollierten Bedingungen als auch auf annähernd unveränderten Standorten deutliche Übereinstimmungen zwischen den Ergebnissen der geophysikalischen Untersuchungen und den tatsächlichen Gegebenheiten im Untergrund vorliegen. Somit hat sich die flächenhafte Kartierung urbaner Baumstandorte als geeignetes Mittel zur Identifikation von Wurzelsystemen erwiesen, wenn der Standort relativ homogen ist, das jeweilige Wurzelsystem oberflächennah und ohne starke vertikale Schwankungen verläuft und typische morphologische Merkmale von Baumwurzeln aufweist (Verzweigung nach außen).

Neben Wurzelsystemen konnte dabei auch typische anthropogene Infrastruktur in Form von Leitungen geortet werden. Daneben ließ sich in einem Fall das Gangsystem zwischen oberflächlich erkennbaren Maulwurfshügeln im Radarergebnis identifizieren. Dabei hat sich die unterschiedliche Morphologie der Objekte als geeignetes Unterscheidungskriterium zu Baumwurzeln erwiesen. 



\section{Literatur}

Alteköster, C.A. (2004). „Untersuchungen zur Reproduzierbarkeit von Bodenradarmessungen“. Diss. Universitäts-und Landesbibliothek Bonn.

Amato, M., B. Basso, G. Celano, G. Bitella, G. Morelli und R. Rossi (2008). „In situ detection of tree root distribution and biomass by multi-electrode resistivity imaging“. In: Tree Physiology 28.10, S. 1441-1448.

Annan, A.P. (2005). "Ground-Penetrating Radar“. In: Near Surface Geophysics. Hrsg. von D.K. Butler. SEG investigations in geophysics series 13. Society of Exploration Geophysics, S. $357-438$.

- (2009). „Electromagnetic principles of ground penetrating radar“. In: Ground Penetrating Radar: Theory and applications. Hrsg. von H.M. Jol. Elsevier Science Ltd. Kap. 1, S. 3-40.

Balder, H. (1998). Die Wurzeln der Stadtbäume. Parey Buchverlag Berlin.

Barsig, M., M. Hirschmann und B. Kluge (2008). Darstellung der Wurzelverläufe und bodenkundliche Feldansprache nahe der Ufermauer des Berliner Landwehrkanals. KUbus. TU Berlin.

Barton, C.V.M. und K.D. Montagu (2004). „Detection of tree roots and determination of root diameters by ground penetrating radar under optimal conditions“. In: Tree Physiology 24.12, S. $1323-1331$.

Butnor, J. R., J. A. Doolittle, L. Kress, S. Cohen und K. H. Johnsen (2001). „Use of groundpenetrating radar to study tree roots in the southeastern United States“. In: Tree Physiology 21.17, S. $1269-1278$.

Butnor, J.R., K. H. Johnsen und R. Oren (2003a). „Reduction of forest floor respiration by fertilization on both carbon dioxide enriched and reference 17 year old loblolly pine stands". In: Global Change Biology 9.6, S. 849-861.

Butnor, J.R., J.A. Doolittle, K.H. Johnsen, L. Samuelson, T. Stokes und L. Kress (2003b). "Utility of ground-penetrating radar as a root biomass survey tool in forest systems“. In: Soil Science Society of America Journal 67.5, S. 1607-1615.

Butnor, J.R., K.H. Johnsen, P. Wikstrom, T. Lundmark und S. Linder (2006). „Imaging tree roots with borehole radar“. In: 11th International Conference on Ground Penetrating Radar. 
Butnor, J.R., C. Barton, F.P. Day, K.H. Johnsen, A.N. Mucciardi, R. Schroeder und D. B. Stover (2012). „Using Ground-Penetrating Radar to Detect Tree Roots and Estimate Biomass“. In: Measuring Roots. Hrsg. von S. Mancuso. Springer. Kap. 12, S. 213-245.

Cassidy, N.J. (2009). „Electrical and magnetic properties of rocks, soils and fluids“. In: Ground Penetrating Radar: Theory and applications. Hrsg. von H.M. Jol. Elsevier Science Ltd. Kap. 2, S. 41-72.

Cermak, J., J. Hruska, M. Martinkova und A. Prax (2000). „Urban tree root systems and their survival near houses analyzed using ground penetrating radar and sap flow techniques". In: Plant and Soil 219.1, S. 103-116.

Chinga, G. und K. Syverud (2007). „Quantification of paper mass distributions within local picking areas". In: Nordic Pulp and Paper Res. J. 22.4, S. 441-446.

Conyers, L. (2004). Ground-Penetrating Radar for Archaeology. Hrsg. von L. Conyers und K. Kvamme. Geophysical Methods for Archaeology. Altamira Press.

Daniels, D.J. (2004). Ground penetrating radar. Hrsg. von N. Stewart und H. Griffiths. 2. Aufl. IEE Radar, sonar, navigations and avionics 15. Wiley Online Library.

Daniels, J. (2000). Ground Penetrating Radar Fundamentals. Appendix to a report to the U.S.EPA, Region V. The Ohio State University.

Dannoura, M., Y. Hirano, T. Igarashi, M. Ishii, K. Aono, K. Yamase und Y. Kanazawa (2008). „Detection of Cryptomeria japonica roots with ground penetrating radar". In: Plant Biosystems - An International Journal Dealing with all Aspects of Plant Biology 142.2, S. 375380.

Doolittle, J.A. und J.R. Butnor (2009). „Soils, Peatlands, and Biomonitoring“. In: Ground penetrating radar: theory and applications. Hrsg. von H.M. Jol. 1. Aufl. Elsevier Science Ltd, S. 179-197.

FGSV (1999). Richtlinie für die Anlage von Straßen - Teil: Landschaftspflege, Abschnitt 4: Schutz von Bäumen, Vegetationsbeständen und Tieren bei Baumaßnahmen.

FLL (2006). Zusätzliche Technische Vertragsbedingungen und Richtlinien für Baumpflege - ZTVBaumpflege. 5. Aufl. Forschungsgesellschaft Landschaftsentwicklung Landschaftsbau e.V. FLL.

Günther, T. und C. Rücker (2011). Boundless Electrical Resistivity Tomography BERT - the user tutorial. 
Goodman, D., S. Piro, K. Schneider, Y. Nishimura, H. Hongo, N. Higashi, J. Steinberg und B. Damiata (2009). „GPR Archaeometry“. In: Ground Penetrating Radar: Theory and applications. Hrsg. von H.M. Jol. Elsevier Science Ltd, S. 479-508.

Gronwald, M. (2010). Kleinräumliche Varianz der Wurzelverteilungen von Buchen und Schwarzkiefernstandorten in urbanen Böden. Unveröffentlichte Bachelorarbeit. Geographisches Institut. Universität Göttingen. Göttingen.

GSSI (2007). RADAN 6.5 User's Manual. North Salem, USA.

Hagrey, S.A. al (2007). „Geophysical imaging of root-zone, trunk, and moisture heterogeneity“. In: Journal of Experimental Botany 58.4, S. 839.

Hagrey, S.A. al und R. Meissner (2004). „Botanical problems studied by applied geophysics,'“ in: Mitteilungen der Deutschen Geophysikalischen Gesellschaft e.V 2, S. 2-8.

Hagrey, S.A. al, R. Meissner, U. Werban, W. Rabbel und A. Ismaeil (2004). „Hydro-, biogeophysics“. In: The Leading Edge. Bd. 23, S. 670.

Hering, E., R. Martin und M. Stohrer (2004). Physik für Ingenieure. Springer.

Hiller, D.A. und H. Meuser (1998). Urbane Böden. Springer.

Hirano, Y., M. Dannoura, K. Aono, T. Igarashi, M. Ishii, K. Yamase, N. Makita und Y. Kanazawa (2008). „Limiting factors in the detection of tree roots using ground-penetrating radar“. In: Plant and Soil 319.1-2, S. 15-24.

Holland, K. (1996). Stadtböden im Keupergbergland am Beispiel Stuttgarts. Hohenheimer Bodenkundliche Hefte 39, S. 228.

Hruska, J., J. Cermak und S. Sustek (1999). „Mapping tree root systems with ground-penetrating radar“. In: Tree Physiology 19.2, S. $125-130$.

Just, A., E. Danckwardt und F. Jacobs (1997). Geoelektrische Tomographie - Prinzip und Anwendungsbeispiele. Universität Leipzig.

Kathage, A., J. Kramp, U. Langer, B. Lehmann, J. Lenz, W. Miegel, E. Räkers und M. Reinhard (2011). Der gläserne Untergrund. Hrsg. von Andreas Kathage. Schriftenreihe aus dem Institut für Rohrleitungsbau Oldenburg 14. Vulkan Verlag, S. 329.

Köstler, J.N., E. Brückner und H. Bibelriether (1968). Die Wurzeln der Waldbäume. P. Parey.

Kutschera, L. und E. Lichtenegger (2002). Wurzelatlas mitteleuropäischer Waldbäume und Sträucher. Stocker. 
Lazzari, L. (2008). „Study of spatial variability of soil root zone properties using electrical resistivity technique“. Diss. University of Basilicata.

Leucci, G. (2011). „The use of three geophysical methods for 3D images of total root volume of soil in urban environments“. In: Exploration Geophysics 41.4, S. 268-278.

Liebetruth, F. und F. Eißfeldt (2008). Baugrundgutachen für die Ufermauern am Landwehrkanal von LWK km 0 bis km 10,74. Techn. Ber. Bundesanstalt für Wasserbau Karlsruhe, Hamburg und Ilmenau im Auftrag des Wasser- und Schifffahrtsamtes Berlin.

Lorra, S. und A. Kathage (2004). „GPR for soil mapping/ assessment and roots imaging“. In: WORKSHOP-Water Use of Woody Crops-techniques, issues, modelling and applications on water management-BOOK of ABSTRACTS/PAPERS.

Meuser, H. (1996). Technogene Substrate als Ausgangsgestein der Böden urban-industrieller Verdichtungsräume: dargestellt am Beispiel der Stadt Essen. Schriftenreihe des Instituts für Pflanzenernährung und Bodenkunde der Universität Kiel, Nr 35.

Meuser, H. und H.P. Blume (2011). „Anthropogene Böden“. In: Handbuch des Bodenschutzes. Hrsg. von H.P. Blume, R. Horn und S. Thile-Bruhn. 4. Aufl. Wiley VCH, S. 465-484.

Naser, M. und A. Junge (2012). „Untersuchungen an Radarmessungen vom Frankfurter Testfeld zur Leitungsortung". In: Sonderband zum Thema: "Georadar-Erfahrungen und Perspektiven". Hrsg. von J. Meßinger (im Auftrag der DGG). Mitteilungen der Deutschen Geophysikalischen Gesellschaft 1, S. 107-111.

Obermeier, P. und C. Sodeikat (2012). „Ultraschall-Untersuchung zur Feststellung von Fehlstellen in Hüllrohren“. In: Berichtsband Fachtagung Bauwerksdiagnose. Hrsg. von Deutsche Gesellschaft für zerstörungsfreie Prüfung.

Oldenburg, D.W. und Y. Li (2005). „Inversion for Applied Geophysics: A Tutorial“. In: Near Surface Geophysics. Hrsg. von D.K. Butler. SEG investigations in geophysics series 13. Society of Exploration Geophysics, S. 89-150.

Olhoeft, G. (2000). „Maximizing the information return from Ground Penetrating Radar“. In: Journal of Applied Geophysics 43, S. 175-187.

Pollmeier, M. (2009). Auflösungseigenschaften und Einsatzmöglichkeiten des Bodenradars im Bereich der zerstörungsfreien Wurzelortung. Unveröffentlichte Bachelorarbeit. HAWK Fakultät R. Göttingen.

Polomski, J. und N. Kuhn (1998). Wurzelsysteme. Hrsg. von Eidg. Forschungsanstalt für Wald, Schnee und Landschaft (WSL/FNP) Birmensdorf. Paul Haupt. 
- (2001). „Wurzelhabitus und Standfestigkeit der Waldbäume“. In: Forstwissenschaftliches Centralblatt 120, S. 303-317.

Raschik, A. (2005). „Untersuchungen von Böden mit hochauflösendem Georadar und Geoelektrik im Modelltrog“. Unveröffentlichte Diplomarbeit. TU Berlin.

Roberts, R., D. Cist und A. Kathage (2009). „Full-resolution GPR imaging applied to utility surveying: insights from multi-polarization data obtained over a test pit“. In: IWAGPR 2009, S. 126-131.

Rossi, R., M. Amato, G. Bitella, R. Bochicchio, J.J. Ferreira Gomes, S. Lovelli, E. Martorella und P. Favale (2010). „Electrical resistivity tomography as a non-destructive method for mapping root biomass in an orchard“. In: European Journal of Soil Science 62, S. 206-215.

Rust, S. und B. Gustke (2006). „Baumschutz am Deich“. In: Baumzeitung 40(2), S. 30-32.

Satriani, A., A. Loperte, M. Proto und M. Bavusi (2010). „Building damage caused by tree roots: laboratory experiments of GPR and ERT surveys“. In: Adv. Geosci 24, S. 133-137.

Sperner, Steffen (1999). „Untersuchungen zur Auflösung von dünnen Schichten mit dem RadarReflexionsverfahren“. Unveröffentlichte Diplomarbeit. TU Berlin.

Stover, D.B., F.P. Day, J.R. Butnor und B.G. Drake (2007). „Effect of elevated $\mathrm{CO}_{2}$ on coarseroot biomass in Florida scrub detected by ground-penetrating radar". In: Ecology 88.5, S. $1328-1334$.

Team, R Core (2012). R: A Language and Environment for Statistical Computing. R Foundation for Statistical Computing. Vienna, Austria.

Topp, G.C., J.L. Davis und A.P. Annan (1980). „Electromagnetic Determination of Soil Water Content: Measurements in Coaxial Transmission Lines“. In: Water Resources Research 16.3, S. 574-582.

Van Vleck, T. (1999). „Ground Penetrating Radar in Tree Root Detection“. Magisterarb. Ohio State University.

Vianden, M. (2009). „Zerstörungsfreie Wurzelortung mit Georadar und elektrischer Widerstandstomographie“. In: Tagungsband Osnabrücker Baumpflegetage 2009.

Vianden, M., D. Bieker und S. Rust (2009). „Beispiele für die zerstörungsfreie Ortung von Baumwurzeln durch geophysikalische Methoden“. In: Jahrbuch der Baumpflege, S. 260-265.

Vianden, M., U. Weihs, F. Kuhnke und S. Rust (2010). „Non-destructive tree root detection with geophysical methods in urban soils“. In: Geophysical Research Abstracts. Bd. 12. EGU. 
- (2011). „Wurzeldetektion mit geophysikalischen Methoden auf urbanen Standorten“. In: Böden verstehen - Böden nutzen - Böden fit machen.

- (2012). „Wurzelortung mit Georadar im städtischen Raum“. In: Sonderband zum Thema: "Georadar- Erfahrungen und Perspektiven". Hrsg. von J. Meßinger (im Auftrag der DGG). Bd. 1. Mitteilungen der Deutschen Geophysikalischen Gesellschaft, S. 101-106.

Weidelt, Peter (2005). „Geoelektrik - Grundlagen Geoelektrik“. In: Methodenhandbuch Deponieuntergrund. Hrsg. von Klaus Knödel, Heinrich Krummel und Gerhard Lange. 2. Bd. Geophysik. Methodenhandbuch Deponieuntergrund. Springer, S. 71-100.

Weihs, U., M. Vianden, F. Kuhnke und A. Koch (2010a). „Zerstörungsfreie Ansicht von Baumwurzelsystemen mit Georadar und Geoelektrik“. In: AFZ- Der Wald 12.

- (2010b). „Zerstörungsfreie Visualisierung von Baumwurzelsystemen“. In: Jahrbuch der Baumpflege, S. 245-252.

Wielopolski, L., G. Hendrey, J. Daniels und M. McGuigan (2000). „Imaging tree root systems in situ“. In: Proceedings of SPIE-The International Society of Optical Engineering. Bd. 4084, S. $642-646$.

Zenone, T., G. Morelli, M. Teobaldelli, F. Fischanger, M. Matteucci, M. Sordini, A. Armani, C. Ferra, T. Chiti und G. Seufert (2008). „Preliminary use of ground-penetrating radar and electrical resistivity tomography to study tree roots in pine forests and poplar plantations". In: Functional Plant Biology 35.10, S. 1047-1058.

Zonge, K., J. Wynn und S. Urquhart (2005). „Resistivity, Induced Polarization, and Complex Resistivity". In: Near Surface Geophysics. Hrsg. von D.K. Butler. SEG investigations in geophysics series 13. Society of Exploration Geophysics, S. 265-300.

Zuur, Alain F., Elena N. Ieno, Neil J. Walker, Anatoly A. Saveliev und Graham M. Smith (2009). Mixed effects models and extensions in ecology with $R$. Springer. 


\section{A Anhang}

\section{A.1 Materialeigenschaften}

Tabelle A.1: Dielektrizitätszahlen verschiedener Materialien. Verändert nach Annan (2005) und Hagrey (2007)

\begin{tabular}{lcc}
\hline Material & $\varepsilon$ & Autor \\
\hline Luft & 1 & Annan \\
Wasser & 81 & \\
trockener Sand & $3-5$ \\
gesättigter Sand & $20-30$ \\
Kalkstein & $4-8$ \\
Schiefer & $5-15$ \\
Silt & $5-30$ \\
Ton & $5-40$ \\
Granit & $4-6$ \\
trockenes Salz & $5-6$ \\
Eis & $3-4$ \\
trockenes Holz (Zellulose) & $4,5 \quad$ Hagrey \\
feuchtes Holz (Zellulose) & 22 \\
\hline & \\
& \\
& \\
&
\end{tabular}


Tabelle A.2: Spezifische Widerstände verschiedener Materialien. Verändert nach Weidelt (2005)

\begin{tabular}{|c|c|c|c|}
\hline \multirow[t]{2}{*}{ Material } & \multicolumn{2}{|c|}{ spezifischer elektrischer Widerstand $(\Omega \mathrm{m})$} & \multirow[t]{2}{*}{ Autor } \\
\hline & Minimum & Maximum & \\
\hline Kies & 50 & $>10^{4}$ & Weidelt \\
\hline Sand & 50 & $>10^{4}$ & \\
\hline Schluff & 20 & 50 & \\
\hline Geschiebemergel & 30 & 70 & \\
\hline Lößlehm & 30 & 100 & \\
\hline Lehm (als Ziegel) & 500 & 5000 & \\
\hline Ton (erdfeucht) & 3 & 30 & \\
\hline Ton (trocken) & & $<1000$ & \\
\hline Torf, Humus, Schlick & 15 & 25 & \\
\hline Mudde, Faulschlamm & 10 & 40 & \\
\hline Moorböden & 10 & 150 & \\
\hline Ölsand (trocken) & $10^{4}$ & $>10^{15}$ & \\
\hline Haus- und Industriemüll & $<1$ & $<1000$ & \\
\hline Sandstein & $<50$ (klüftig, feucht) & $>10^{5}$ & \\
\hline Kalkstein & 100 (klüftig, feucht) & $>10^{5}$ & \\
\hline Tonschiefer & 50 (klüftig, feucht) & $>10^{5}$ & \\
\hline Magmatite, Metamorphite & 150 (verwittert, feucht) & $>10^{5}$ & \\
\hline Schwarzschiefer & $<1$ & 50 & \\
\hline Steinsalz & 30 (feucht) & $>10^{6}$ & \\
\hline Destilliertes Wasser & & $>10^{3}$ & \\
\hline Schneefirn & & $>10^{5}$ & \\
\hline Natürliche Wässer & 10 & 300 & \\
\hline Salzlaugen & $<0,1$ & & \\
\hline
\end{tabular}




\section{A.2 Aufbau der Versuchsbecken}

Tabelle A.3: Anordnung der Wurzelattrappen und Probekörper in den Versuchsbecken 1 bis 4

\begin{tabular}{|c|c|c|c|c|c|c|c|c|}
\hline & $\dot{z}$ & 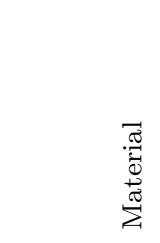 & 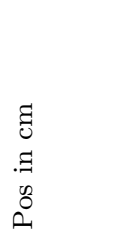 & 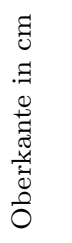 & 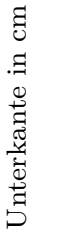 & 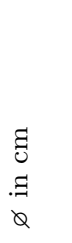 & 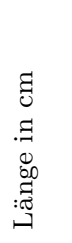 & 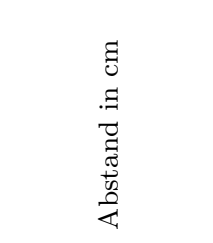 \\
\hline \multirow[t]{9}{*}{ Becken 1} & 1 & Platane & 50 & 28,0 & 40,0 & 12,0 & 50,0 & zum Rand: 50,0 \\
\hline & 2 & & 100 & 27,5 & 40,0 & 12,5 & 18,0 & $\mathrm{zu} 3: 50,0$ \\
\hline & 3 & & 150 & 32,0 & 40,0 & 8,0 & 50,0 & $\mathrm{zu} 4: 50,0$ \\
\hline & 4 & & 200 & 34,0 & 40,0 & 6,0 & 50,0 & $\mathrm{zu} 5: 50,0$ \\
\hline & 5 & & 250 & 36,0 & 40,0 & 4,0 & 50,0 & zu 6: 50,0 \\
\hline & 6 & & 300 & 37,0 & 40,0 & 3,0 & 50,0 & $\mathrm{zu} 7: 50,0$ \\
\hline & 7 & & 350 & 38,0 & 40,0 & 1,8 & 50,0 & zu 8: 50,0 \\
\hline & 8 & & 400 & 38,0 & 40,0 & 1,5 & 50,0 & zum Rand: 50,0 \\
\hline & 9 & Tonkinstab & 450 & 39,0 & 40,0 & 0,8 & 50,0 & zu 9: 50,0 \\
\hline \multirow[t]{16}{*}{ Becken 2} & 1 & Platane & 50 & 28,0 & 40,0 & 12,0 & 50,0 & zum Rand: 50,0 \\
\hline & 2 & & 110 & 35,0 & 40,0 & 5,0 & 50,0 & $\mathrm{zu} 3: 30,0$ \\
\hline & 3 & & 140 & 36,0 & 40,0 & 4,0 & 50,0 & $\mathrm{zu} 4: 15,0$ \\
\hline & 4 & & 155 & 36,0 & 40,0 & 4,0 & 48,0 & zu $5: 10,0$ \\
\hline & 5 & & 165 & 36,0 & 40,0 & 4,0 & 48,0 & zu 6: 10,0 \\
\hline & 6 & & 175 & 35,5 & 40,0 & 4,5 & 51,0 & zu $7: 10,0$ \\
\hline & 7 & & 185 & 34,5 & 40,0 & 5,5 & 48,0 & zu 8: 50,0 \\
\hline & \multicolumn{8}{|l|}{8} \\
\hline & $\mathrm{a}$ & & 235 & 10,0 & 15,0 & 5,0 & 48,0 & zu 9: 50,0 \\
\hline & $\mathrm{b}$ & & 235 & 22,0 & 28,0 & 6,0 & 50,0 & zu 9: 50,0 \\
\hline & c & & 400 & 35,0 & 40,0 & 5,0 & 47,0 & zu 9: 50,0 \\
\hline & 9 & & 285 & 5,0 & 40,0 & 5,0 & 50,0 & zu 10: 50,0 \\
\hline & 10 & & 335 & 5,0 & 40,0 & 8,0 & 35,0 & zu 11: 50,0 \\
\hline & \multicolumn{8}{|l|}{11} \\
\hline & $\mathrm{a}$ & & $385-415$ & 34,0 & 40,0 & 6,0 & 50,0 & zu 12: 40,0 \\
\hline & $\mathrm{b}$ & & $385-415$ & 34,0 & 40,0 & 4,5 & 50,0 & zu 12: 40,0 \\
\hline
\end{tabular}




\begin{tabular}{|c|c|c|c|c|c|c|c|c|}
\hline & $\dot{\vec{z}}$ & 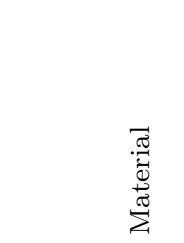 & $\begin{array}{l}\text { घ्व } \\
. \Xi \\
\text { D } \\
0\end{array}$ & 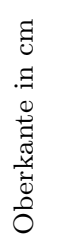 & 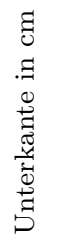 & $\begin{array}{l}\Xi \\
0 \\
. \Xi \\
Q\end{array}$ & 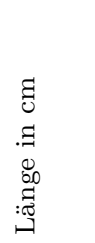 & 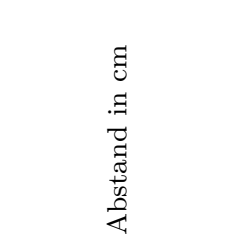 \\
\hline \multirow[t]{3}{*}{ Becken 2} & 12 & & & & & & & \\
\hline & $\mathrm{a}$ & & 455 & 6,0 & 10,0 & 4,0 & 50,0 & zum Rand: 45,0 \\
\hline & $\mathrm{b}$ & & 455 & 36,0 & 40,0 & 4,0 & 50,0 & zum Rand: 45,0 \\
\hline \multirow[t]{16}{*}{ Becken 3} & 1 & Esche & $30-140$ & 37,0 & 40,0 & 3,0 & 110,0 & zum Rand: 50,0 \\
\hline & 2 & Kunststoff & 180 & 29,0 & 40,0 & 11,0 & 40,0 & $\mathrm{zu} 3: 50,0$ \\
\hline & 3 & Platane & 210 & 37,0 & 40,0 & 3,0 & 35,0 & $\mathrm{zu} 4: 50,0$ \\
\hline & 4 & Eisen & 240 & 36,0 & 40,0 & 4,0 & 50,0 & zu 5: 50,0 \\
\hline & 5 & Platane & 265 & 37,0 & 40,0 & 3,0 & 40,0 & $\mathrm{zu} 6: 50,0$ \\
\hline & 6 & Steinarten: & & & & & & \\
\hline & $\mathrm{a}$ & Basalt & 300 & 29,0 & 15,0 & $11,0 \times 11,0 \times 11,0$ & 50,0 & zu 9: 50,0 \\
\hline & $\mathrm{b}$ & Betonstein & 300 & 29,0 & 28,0 & $21,0 \times 11,0 \times 8,0$ & 50,0 & zu 9: 50,0 \\
\hline & $\mathrm{c}$ & gebr. Ziegel & 300 & 28,0 & 40,0 & $25,0 \times 12,0 \times 7,0$ & 50,0 & zu 9: 50,0 \\
\hline & 7 & Eisen & 340 & 39,0 & 40,0 & 1,0 & 50,0 & $\mathrm{zu} 8: 15,0$ \\
\hline & 8 & Eisen & 355 & 39,0 & 40,0 & 1,0 & 50,0 & zu 9: 25,0 \\
\hline & 9 & Gummi & 380 & 37,0 & 40,0 & 3,0 & 50,0 & zu 10: 25,0 \\
\hline & 10 & Elektrokabel & 405 & 39,0 & 40,0 & 1,0 & 50,0 & zu 10: 15,0 \\
\hline & 11 & Draht & 420 & 39,7 & 40,0 & 0,3 & 50,0 & $\mathrm{zu} 12: 20,0$ \\
\hline & 12 & Bauholz & 440 & 33,5 & 40,0 & 6,5 & 50,0 & zu 13: 20,0 \\
\hline & 14 & Vlies & 460 & 39,0 & 40,0 & $20,0 \times 40,0 \times 1,0$ & 50,0 & zum Rand: 40,0 \\
\hline \multirow[t]{7}{*}{ Becken 4} & 1 & Rotbuche & 60 & 10,0 & 40,0 & 30,0 & 48,0 & zum Rand: 60,0 \\
\hline & 2 & & 140 & 12,0 & 40,0 & 28,0 & 53,0 & zu 3: 80,0 \\
\hline & 3 & & 215 & 21,0 & 40,0 & 19,0 & 53,0 & $\mathrm{zu} 4: 75,0$ \\
\hline & 4 & & 280 & 24,0 & 40,0 & 16,0 & 54,0 & zu 5: 65,0 \\
\hline & 5 & Platane & 340 & 34,0 & 40,0 & 6,0 & 61,0 & zu 6: 60,0 \\
\hline & 6 & & 400 & 14,0 & 40,0 & 6,0 & 51,0 & zu 7: 50,0 \\
\hline & 7 & & 450 & 9,0 & 40,0 & 6,0 & 51,0 & zm Rand: 50,0 \\
\hline
\end{tabular}




\section{A.3 Lage und Fotos der Messflächen}

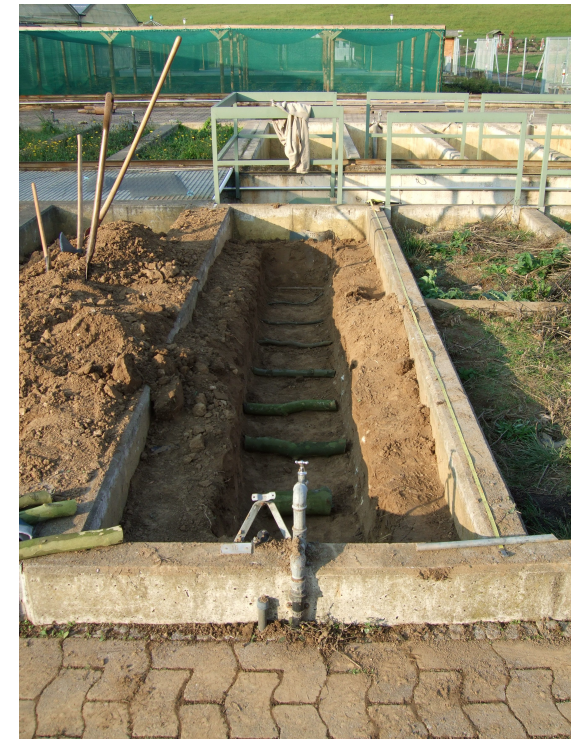

a) Becken 1

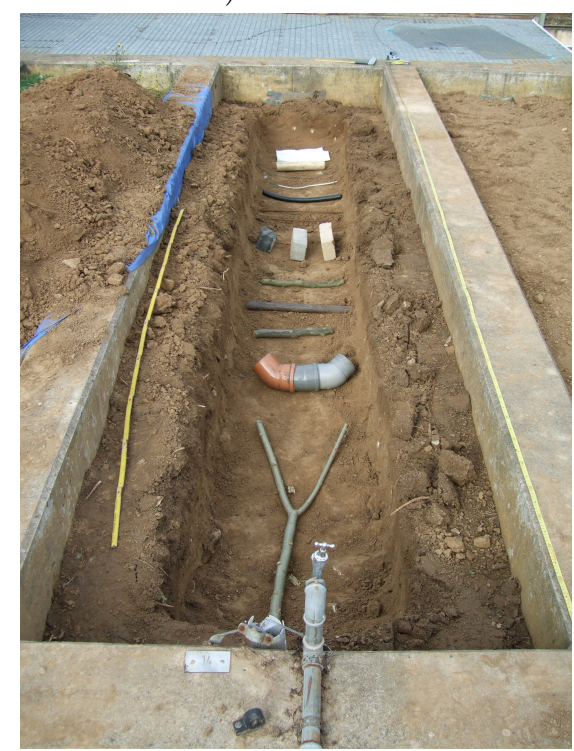

c) Becken 3

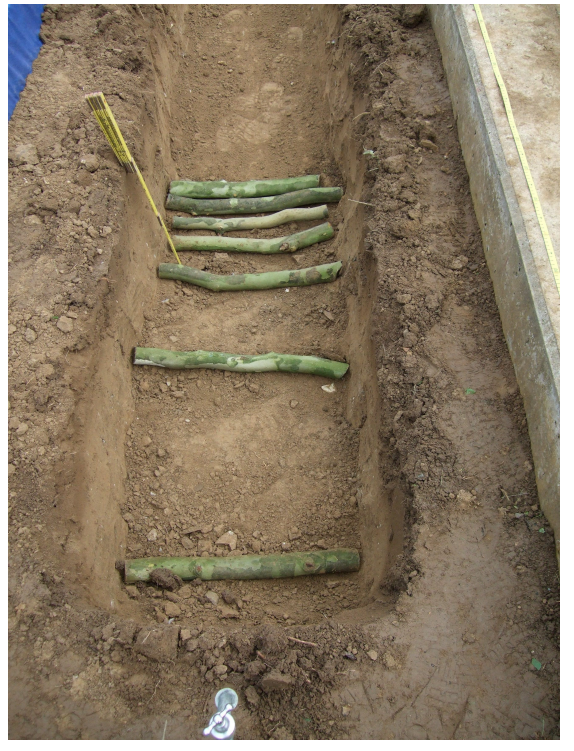

b) Becken 2

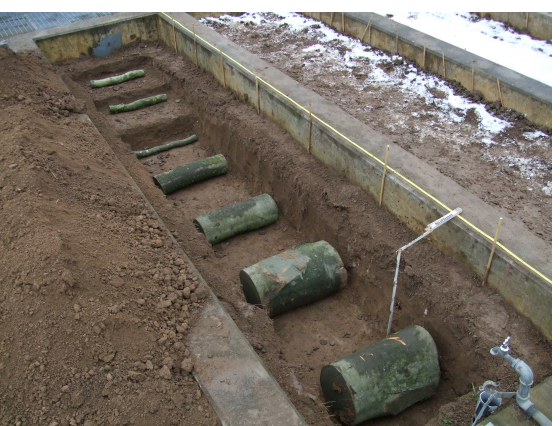

d) Becken 4

Abbildung A.1: Versuchsbecken im Neuen Botanischen Garten. 
A Anhang

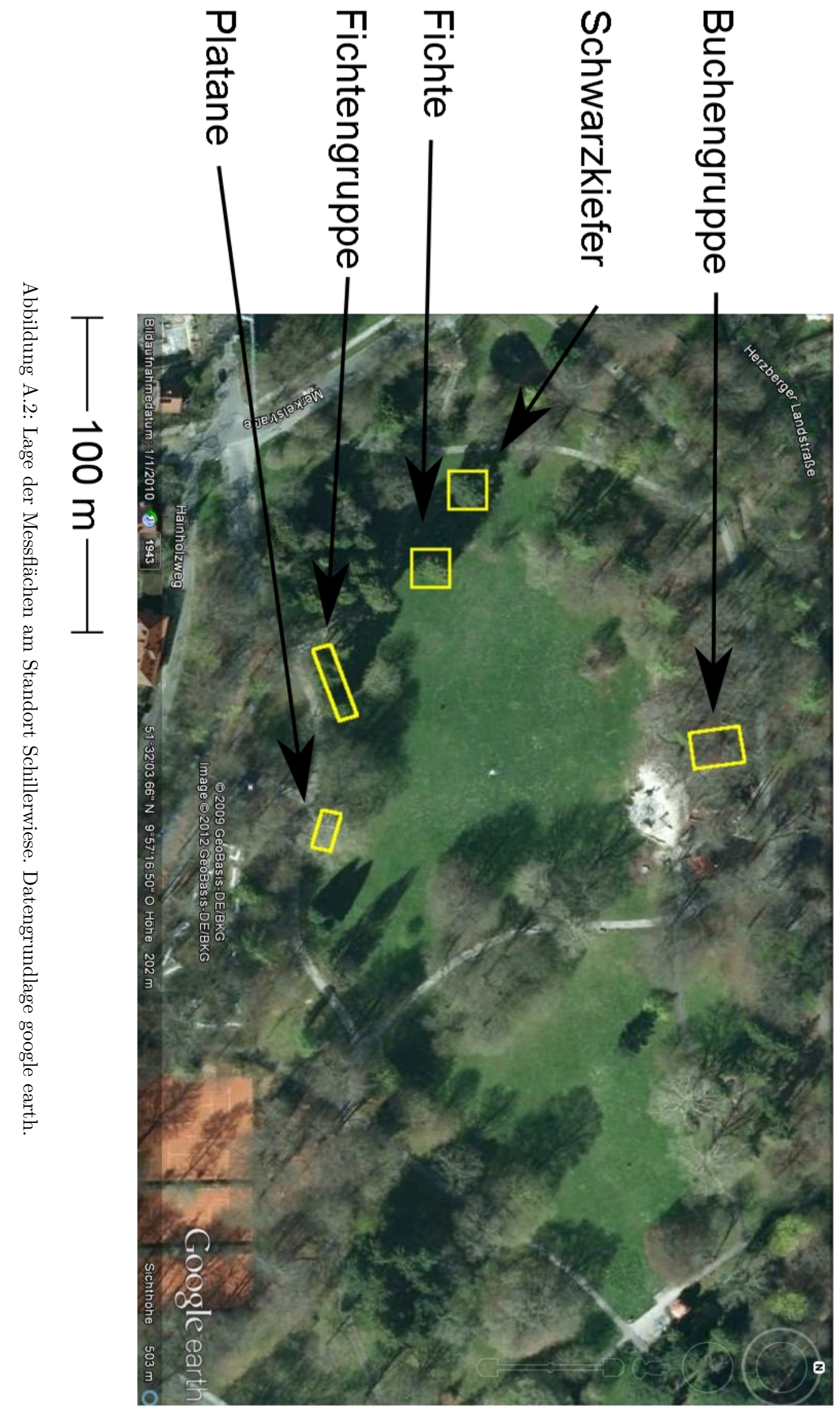




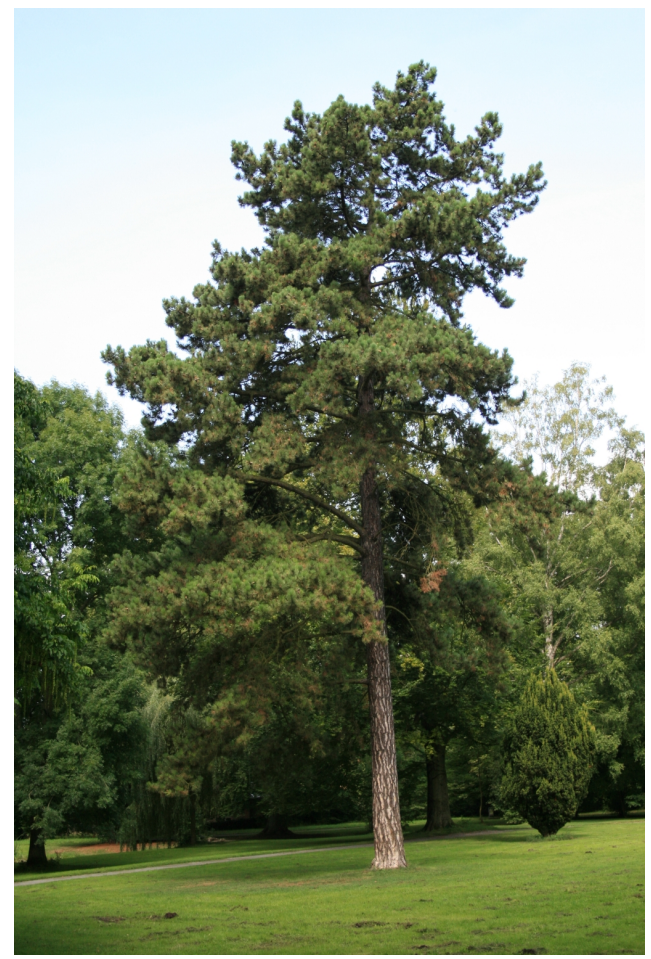

a) Schwarzkiefer

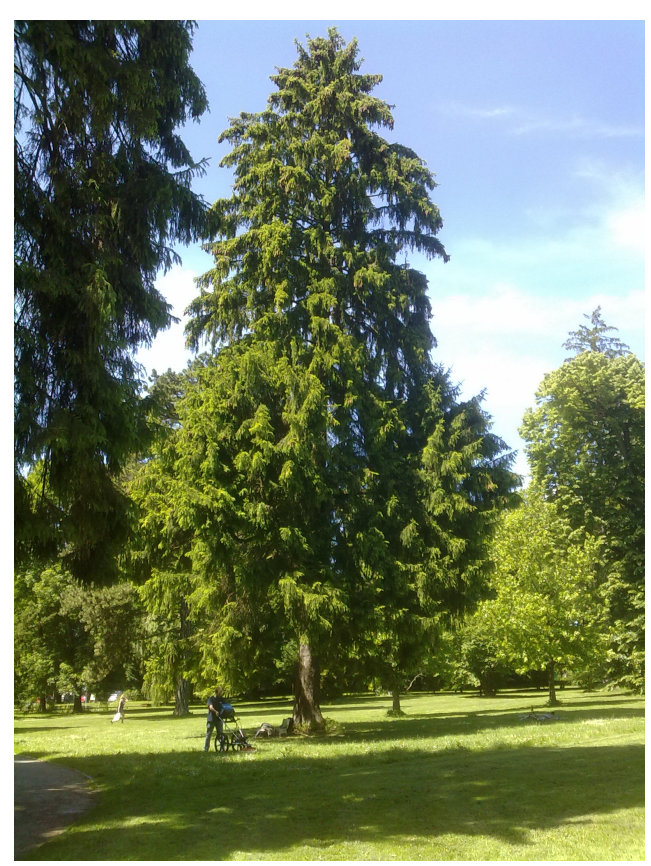

c) Fichte

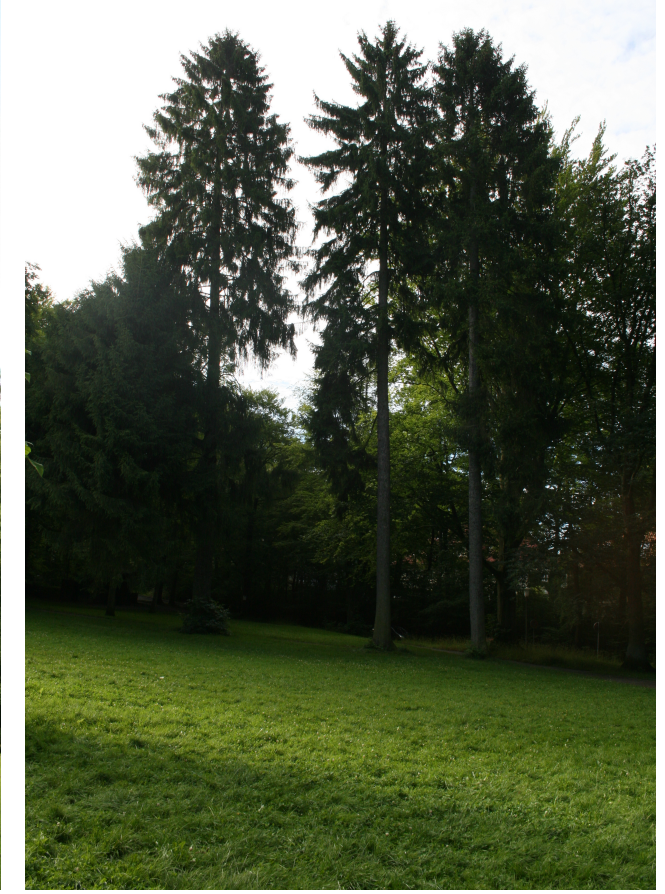

b) Fichtengruppe

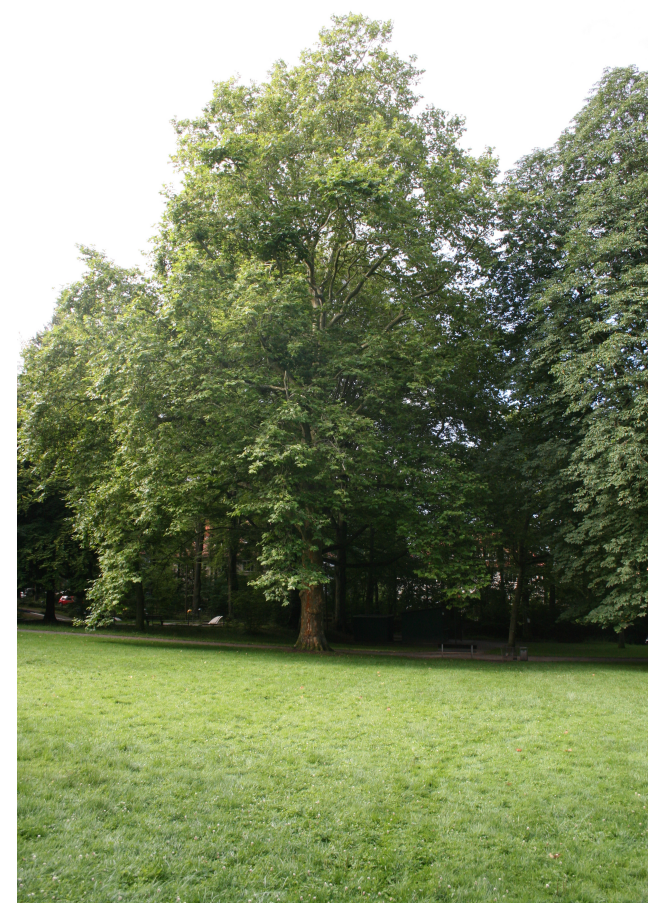

d) Platane

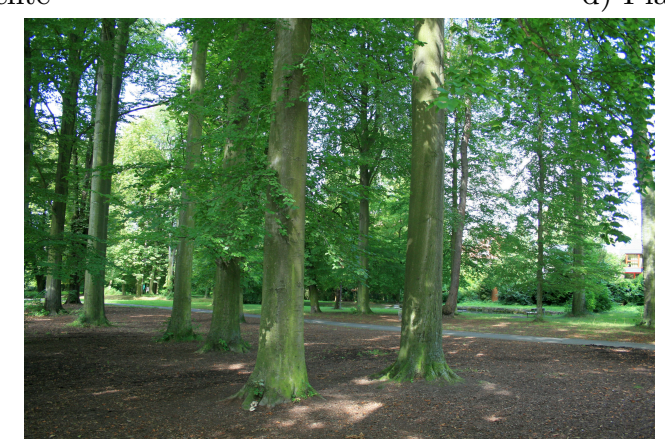

e) Buchengruppe

Abbildung A.3: Untersuchte Bäume auf der Schillerwiese. 


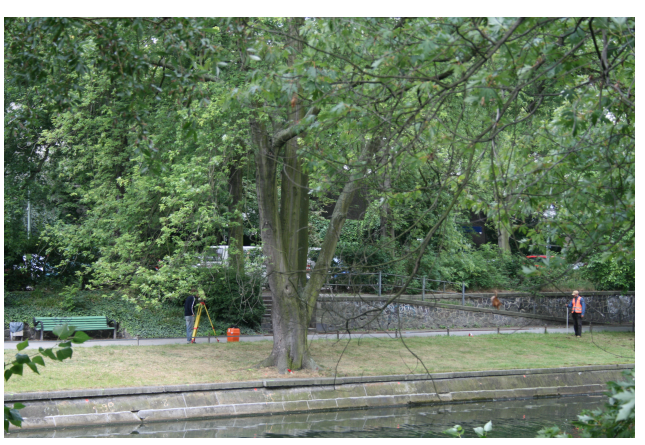

a) Silberahorn

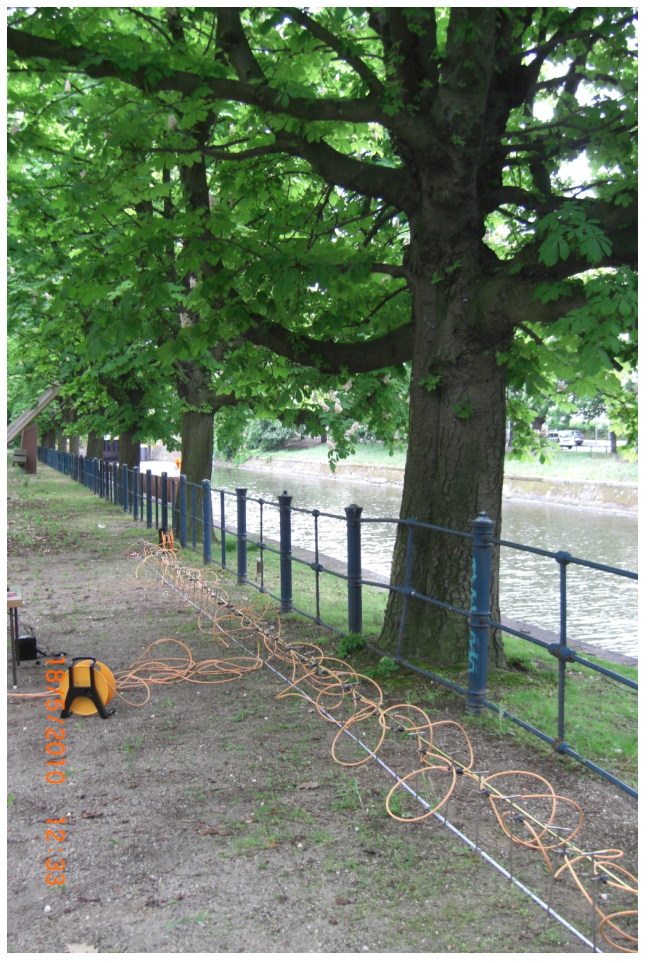

c) Große Kastanie

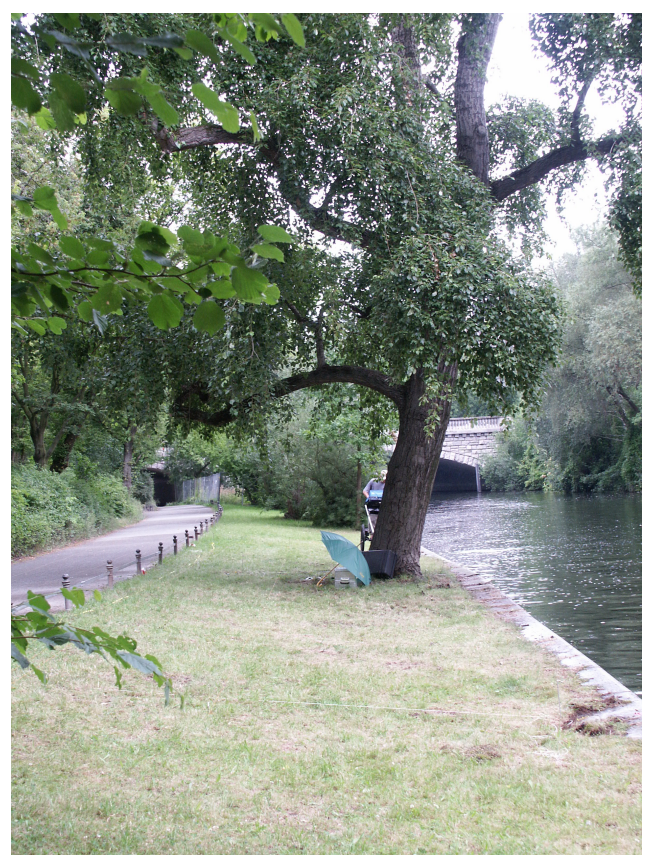

b) Hybridpappel

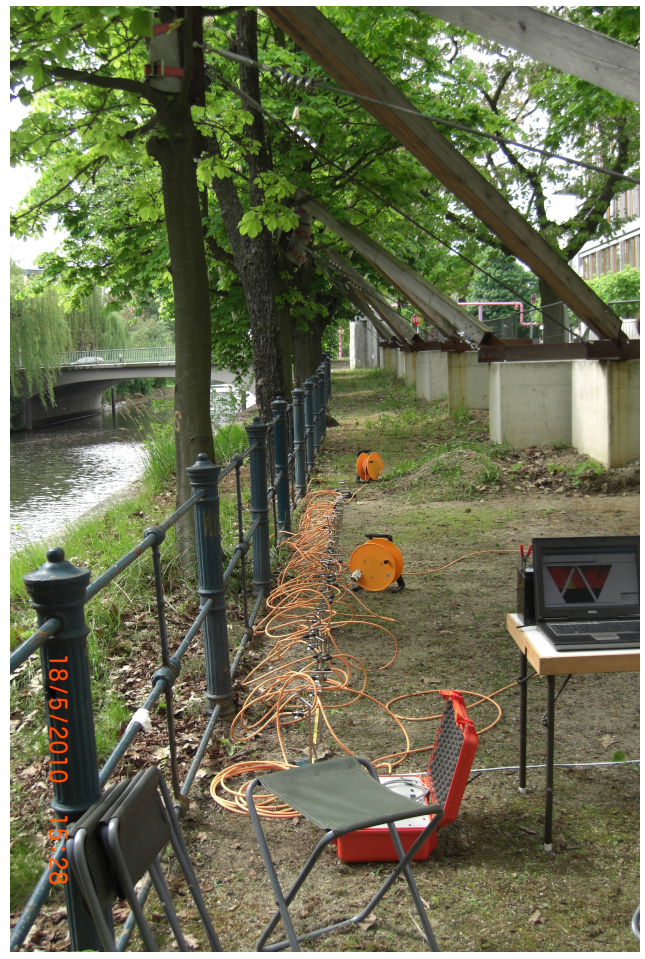

d) Kleine Kastanie

Abbildung A.4: Untersuchte Bäume am Berliner Landwehrkanal. Einsteinufer (oben); Corneliusufer (unten). 


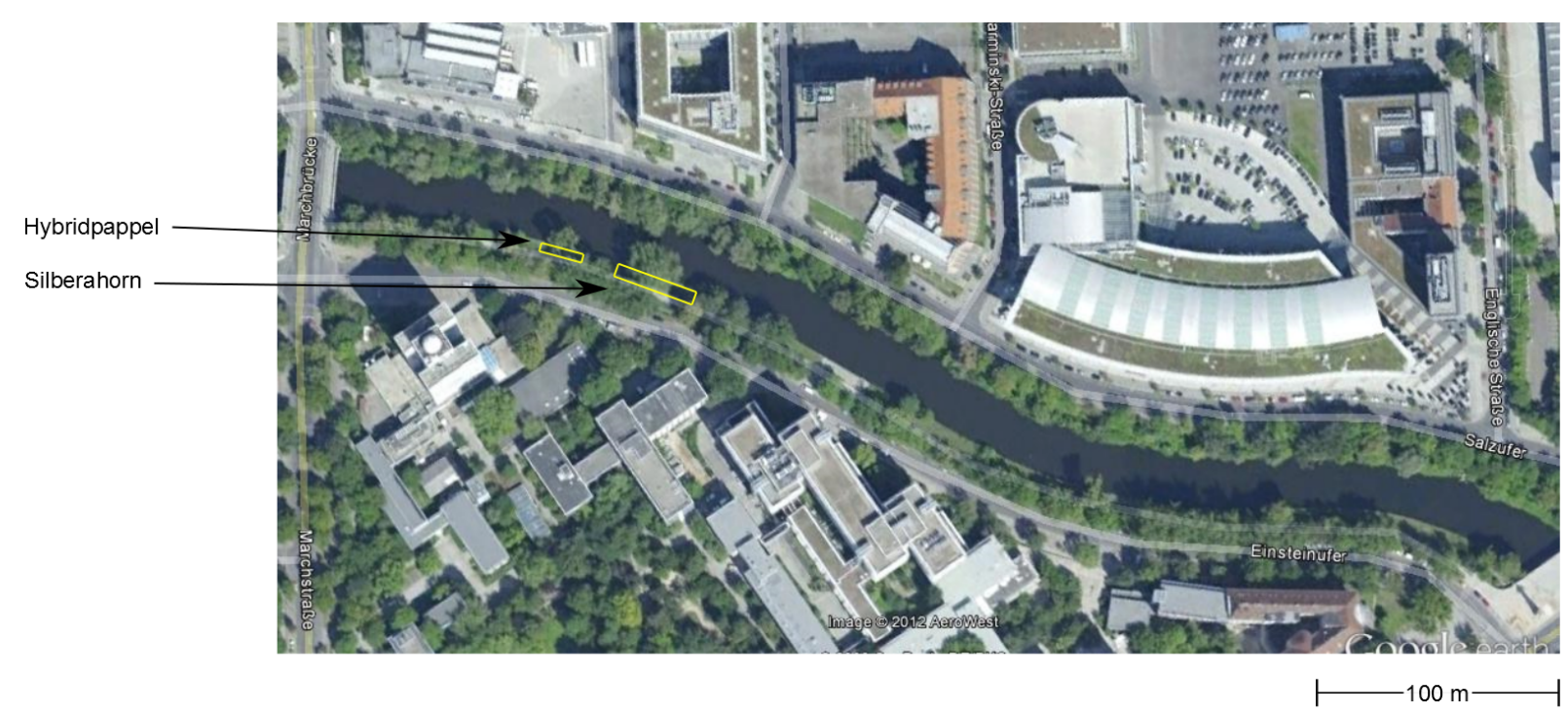

A $(M u+f s, u, h)$ Auffüllung mit geringer bis mittlerer Festigkeit

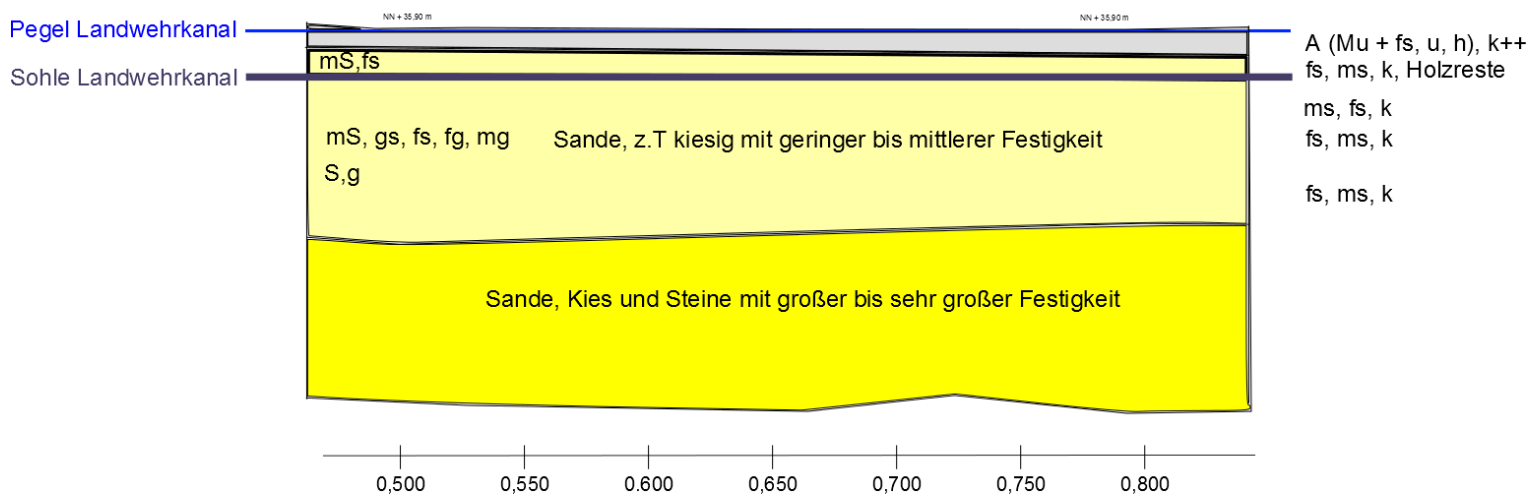

Abbildung A.5: Lage der Messflächen am Standort Landwehrkanal, Einsteinufer. Datengrundlage google earth (oben), geologischer Längsschnitt- verändert nach Liebetruth und Eißfeldt 2008 (unten). 

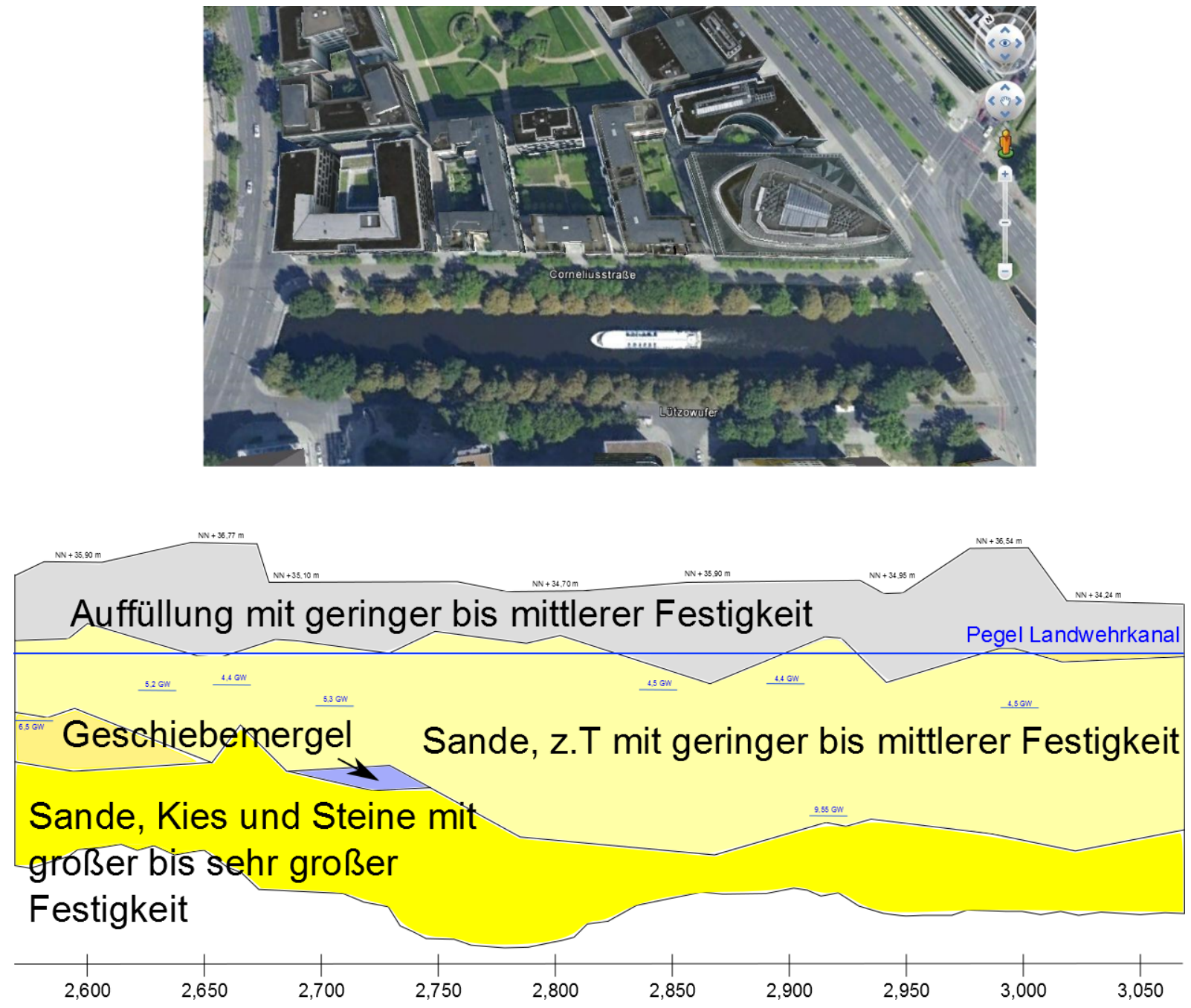

Abbildung A.6: Lage der Messflächen am Standort Landwehrkanal, Corneliusufer. Datengrundlage google earth (oben), geologischer Längsschnitt- verändert nach Liebetruth und Eißfeldt 2008 (unten). 


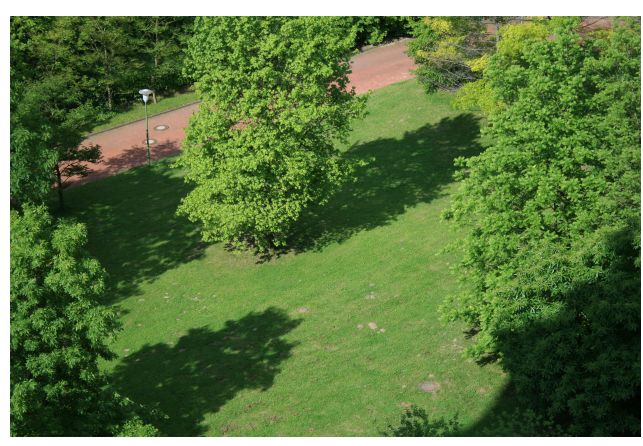

a) Amberbaum Göttingen

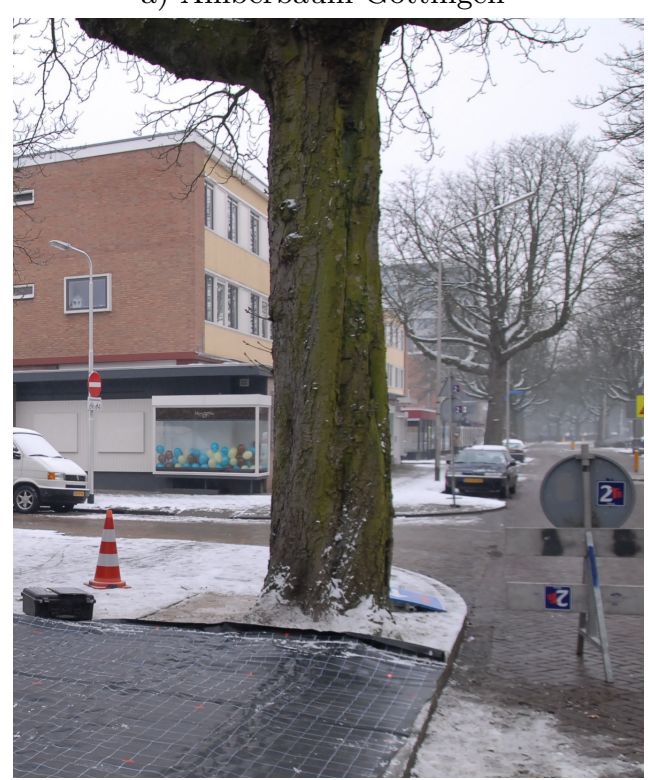

c) Kastanie Nimwegen

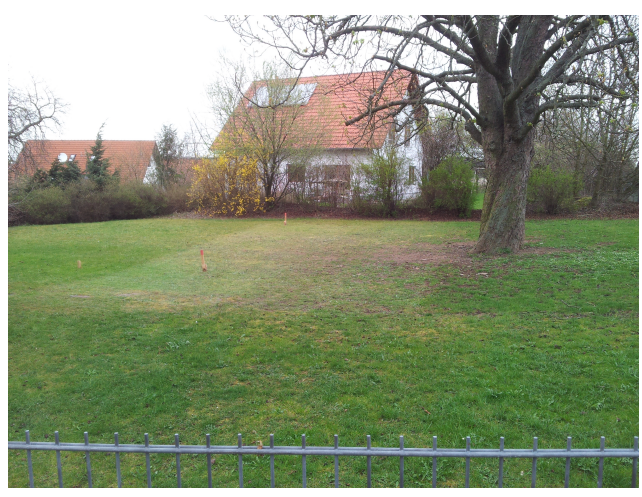

b) Kastanie Fulda

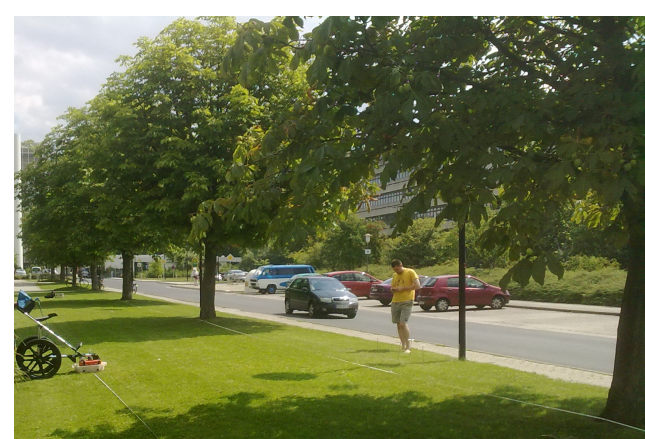

d) Kastanien Physik Göttingen

Abbildung A.7: Untersuchte Bäume auf den weiteren Messflächen. 


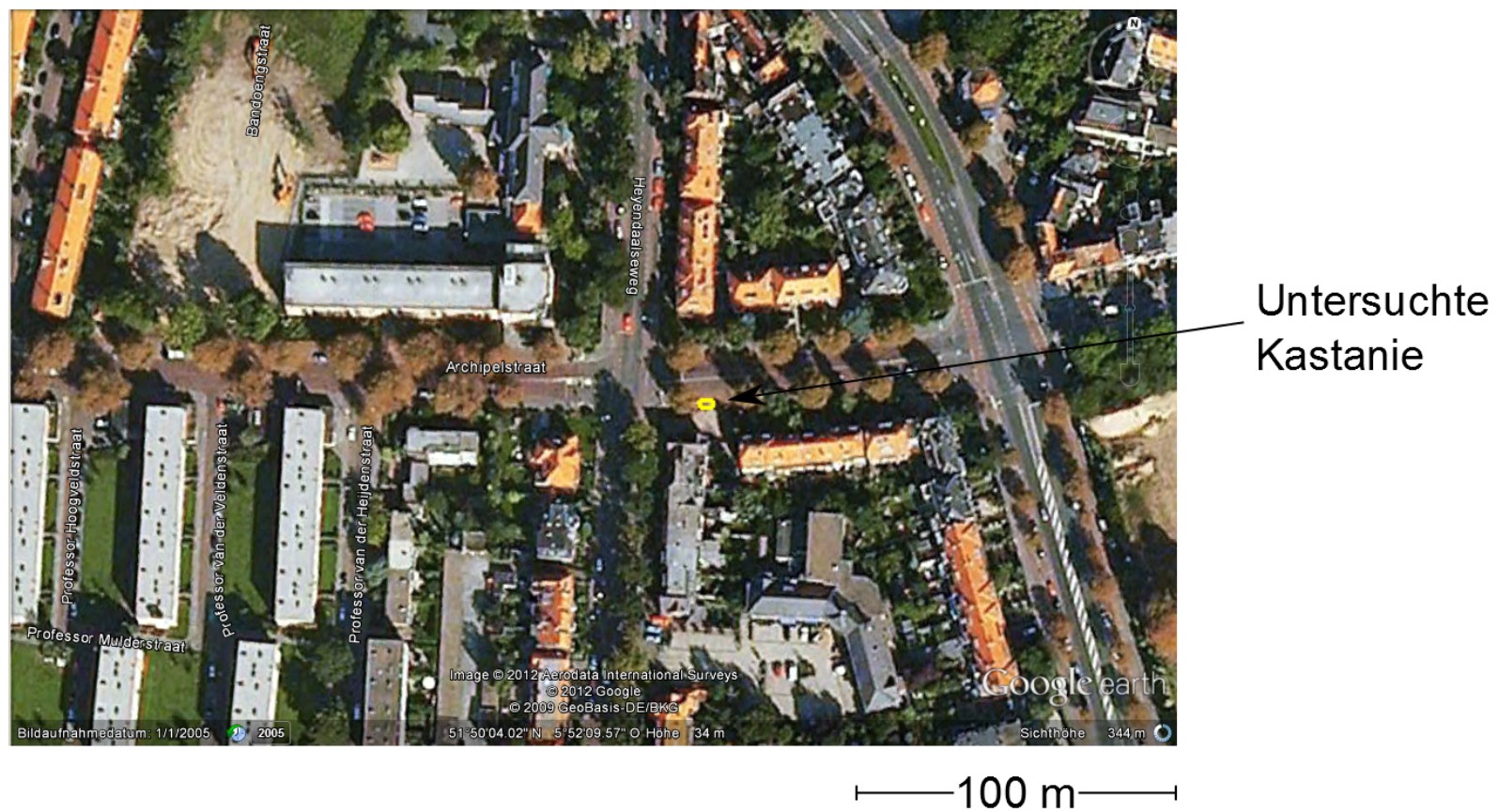

Abbildung A.8: Lage der Messfläche am Standort Kastanie Archipelstraat Nimwegen. Datengrundlage google earth.

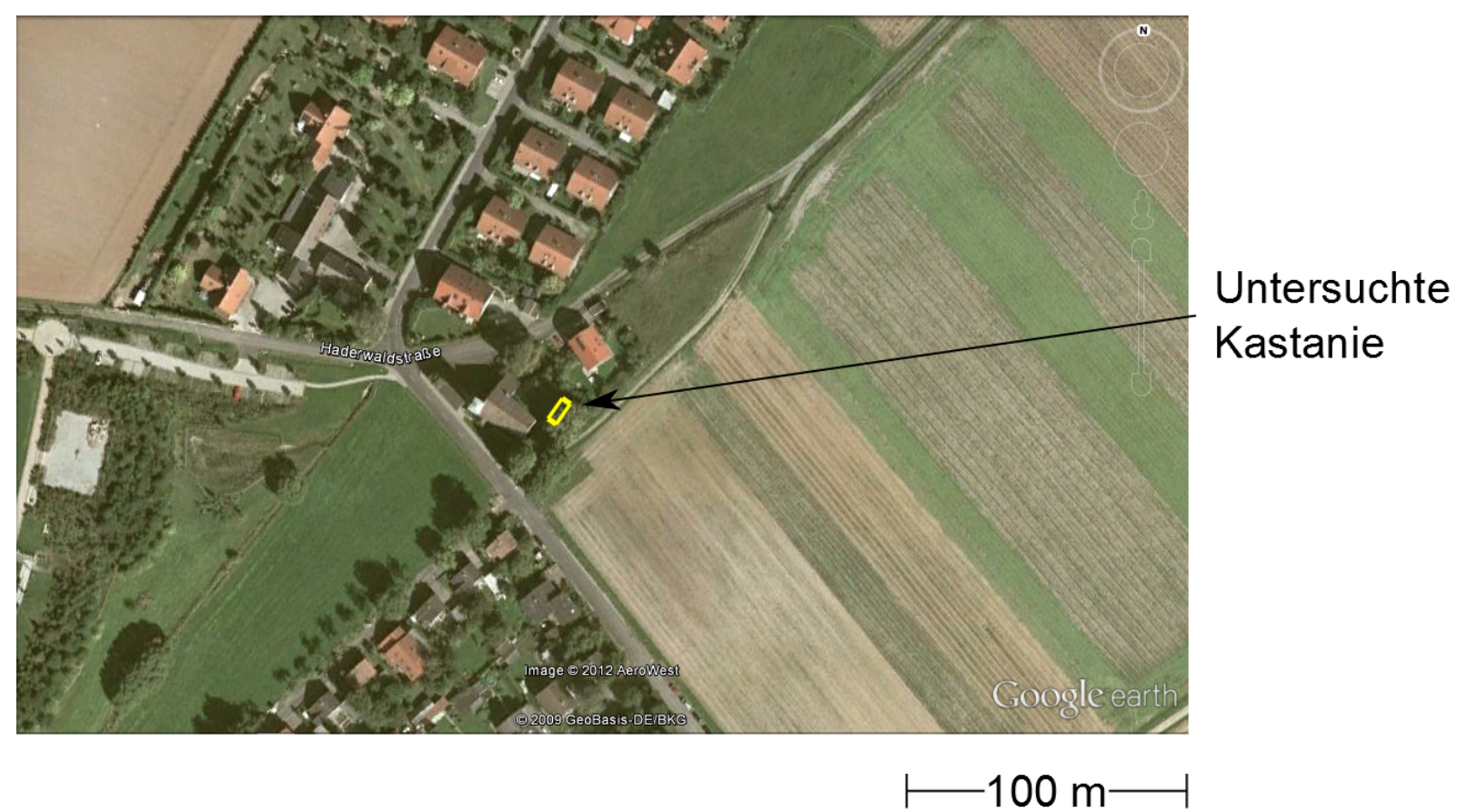

Abbildung A.9: Lage der Messfläche am Standort Kastanie Kreuzkirche Fulda. Datengrundlage google earth. 


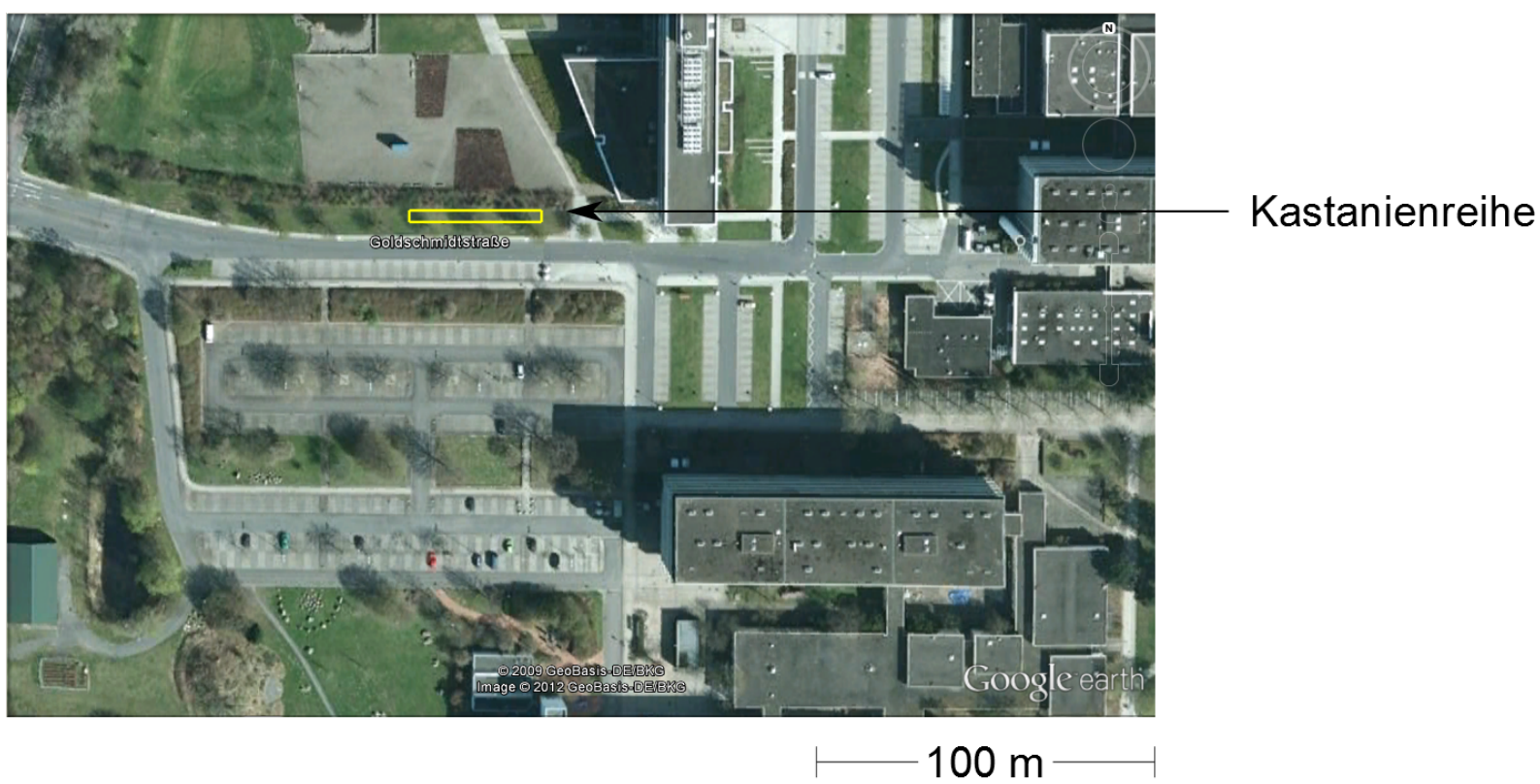

Abbildung A.10: Lage der Messfläche am Standort Kastanienreihe Göttingen. Datengrundlage google earth.

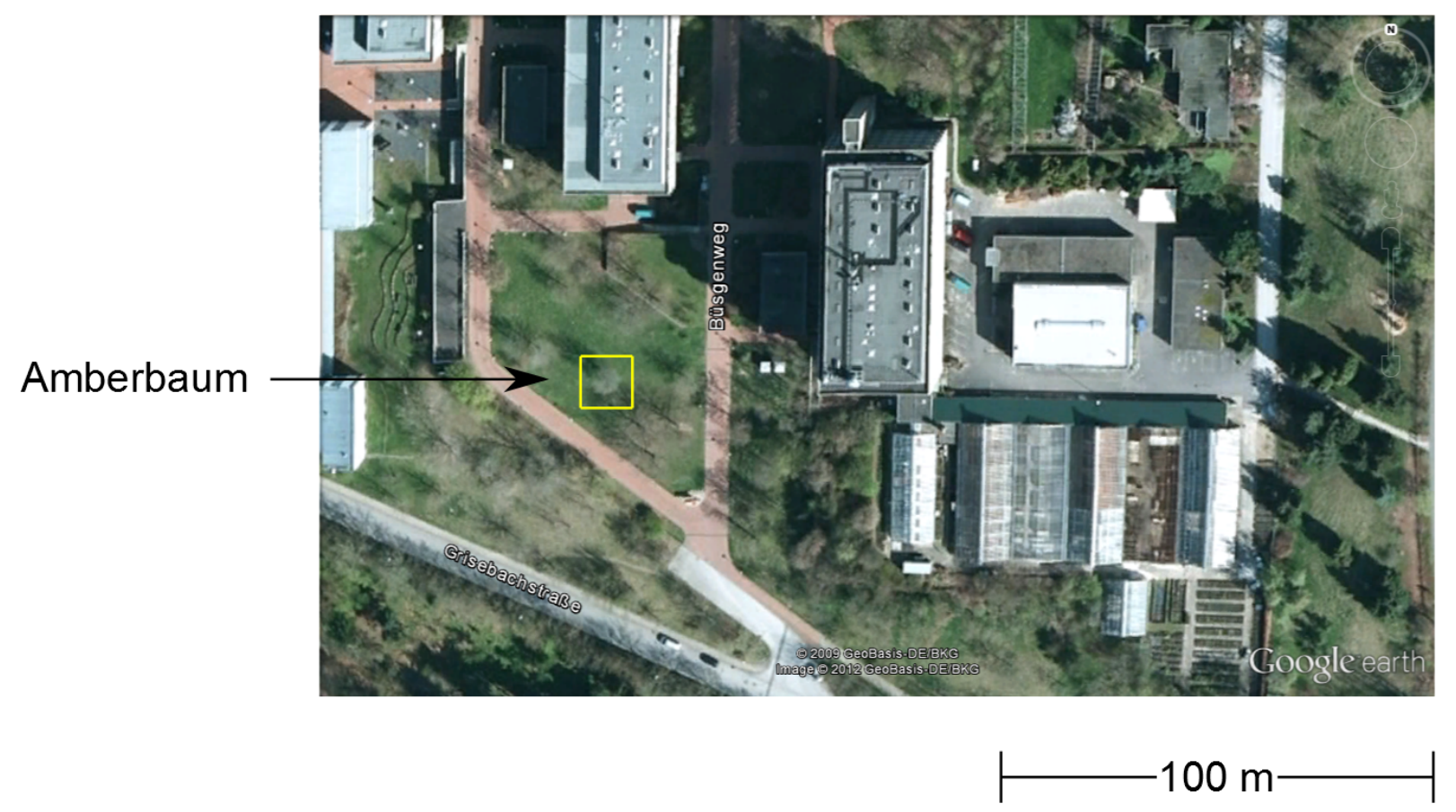

Abbildung A.11: Lage der Messfläche Amberbaum Göttingen. Datengrundlage google earth. 


\section{A.4 Gesamtradargramme und -tomogramme der einzelnen Messflächen und} weitere Beispiele

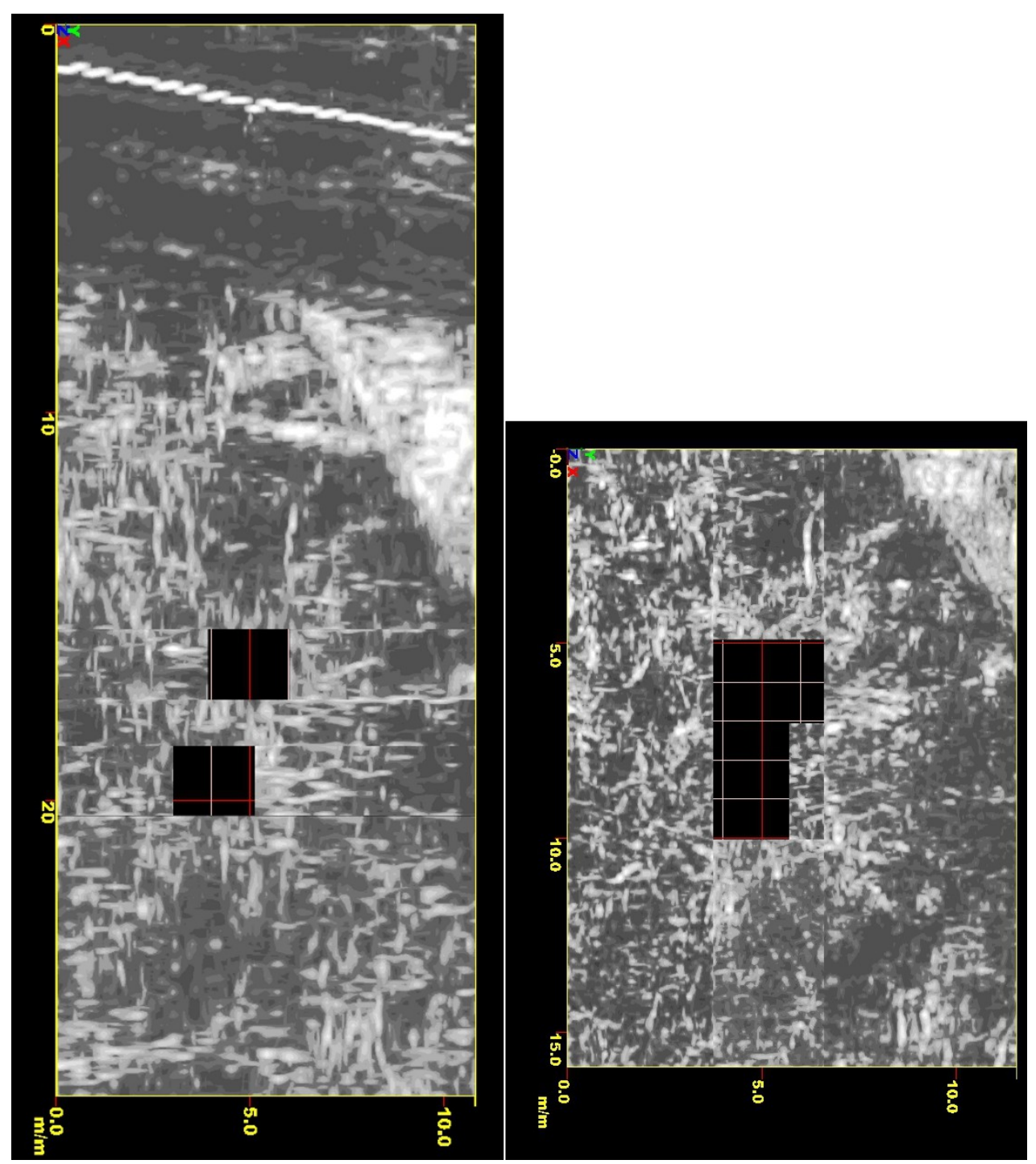

Abbildung A.12: Reproduzierbarkeitsmessung auf der Messfläche Buchengruppe mit einem Rasterabstand von $15 \mathrm{~cm}$ (oben) und $30 \mathrm{~cm}$ (unten). 


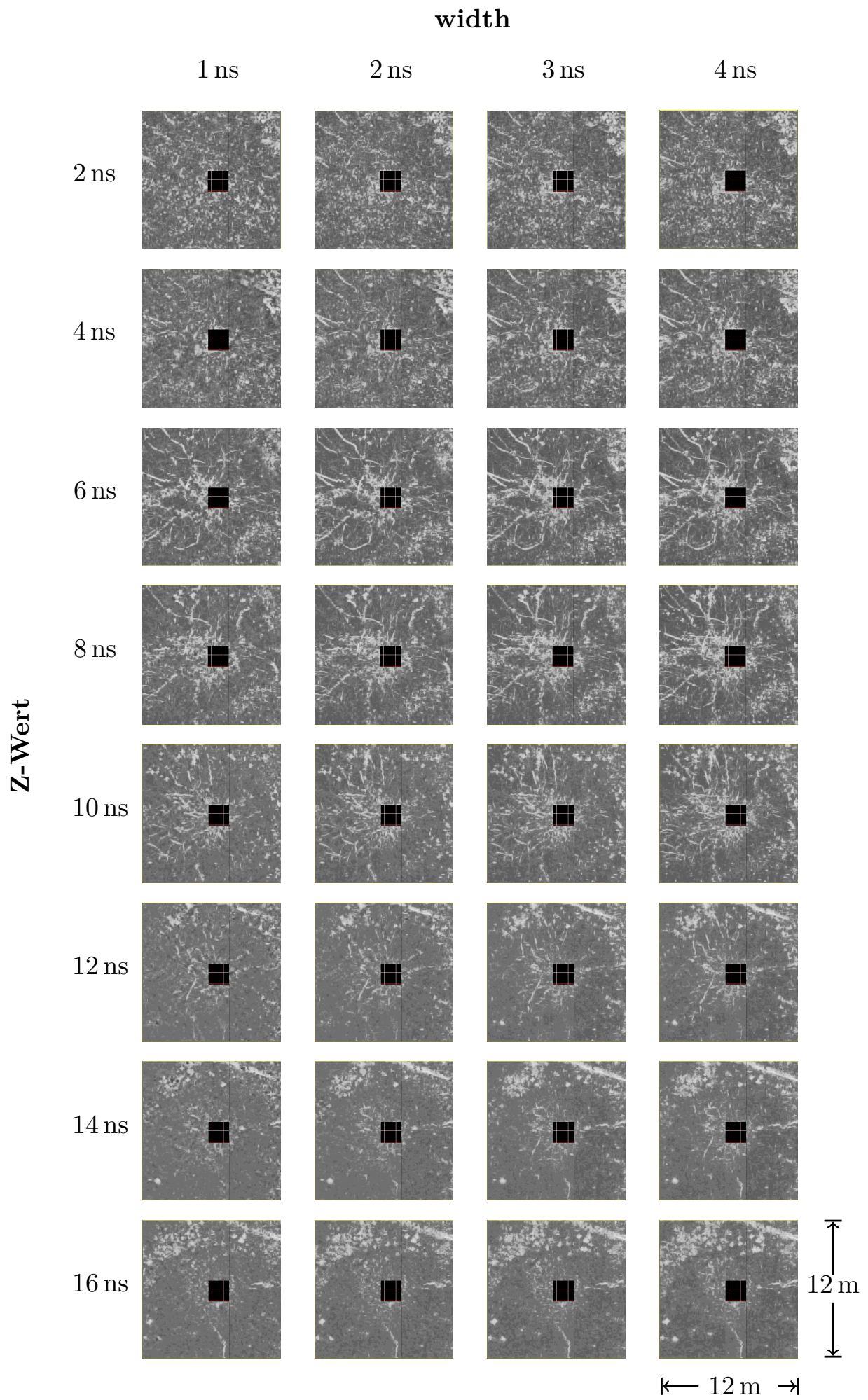

Abbildung A.13: Ergebnis der Georadaruntersuchung auf der Messfläche Schwarzkiefer. 


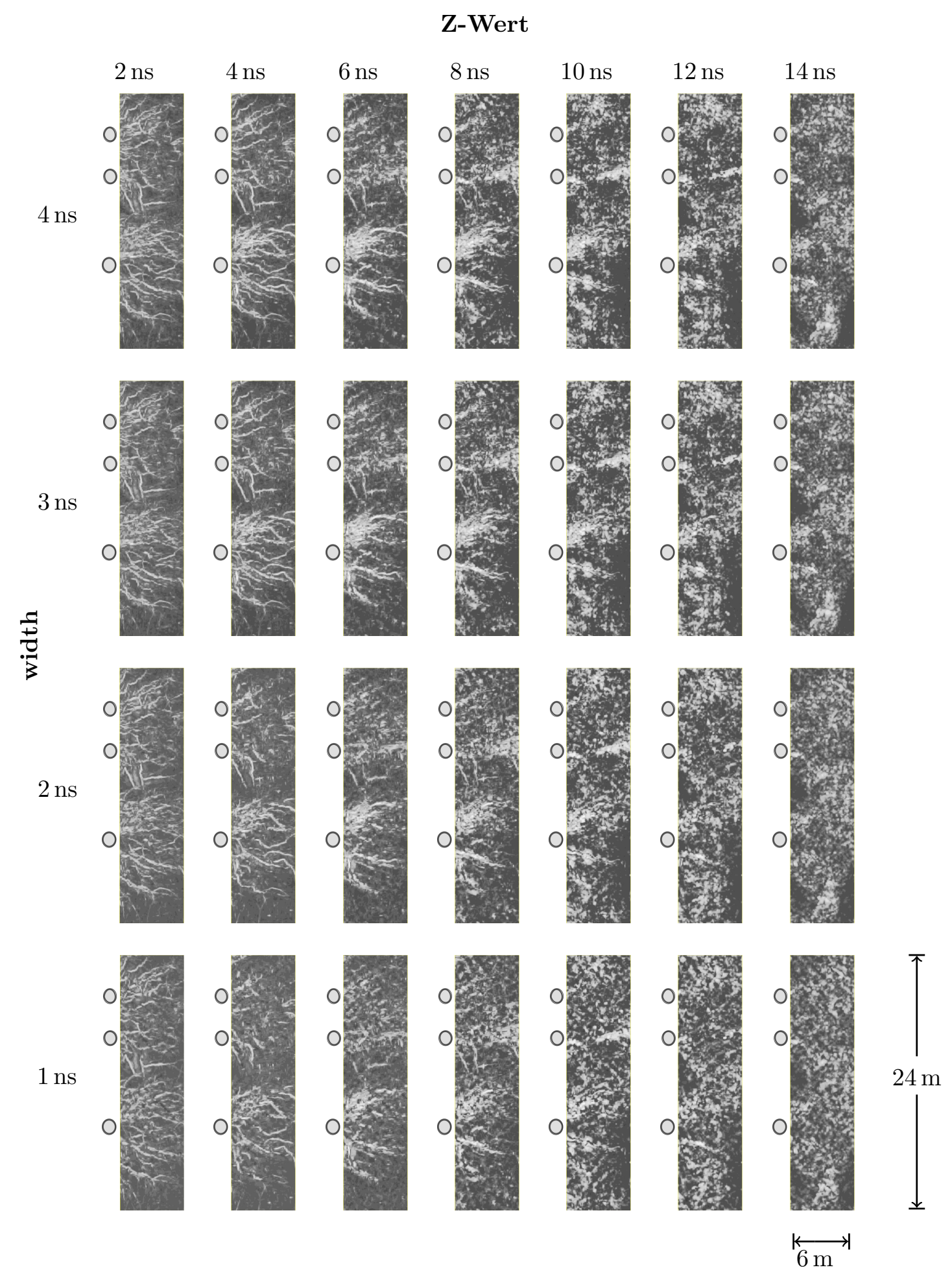

Abbildung A.14: Ergebnis der Georadaruntersuchung auf der Messfläche Fichtengruppe. 


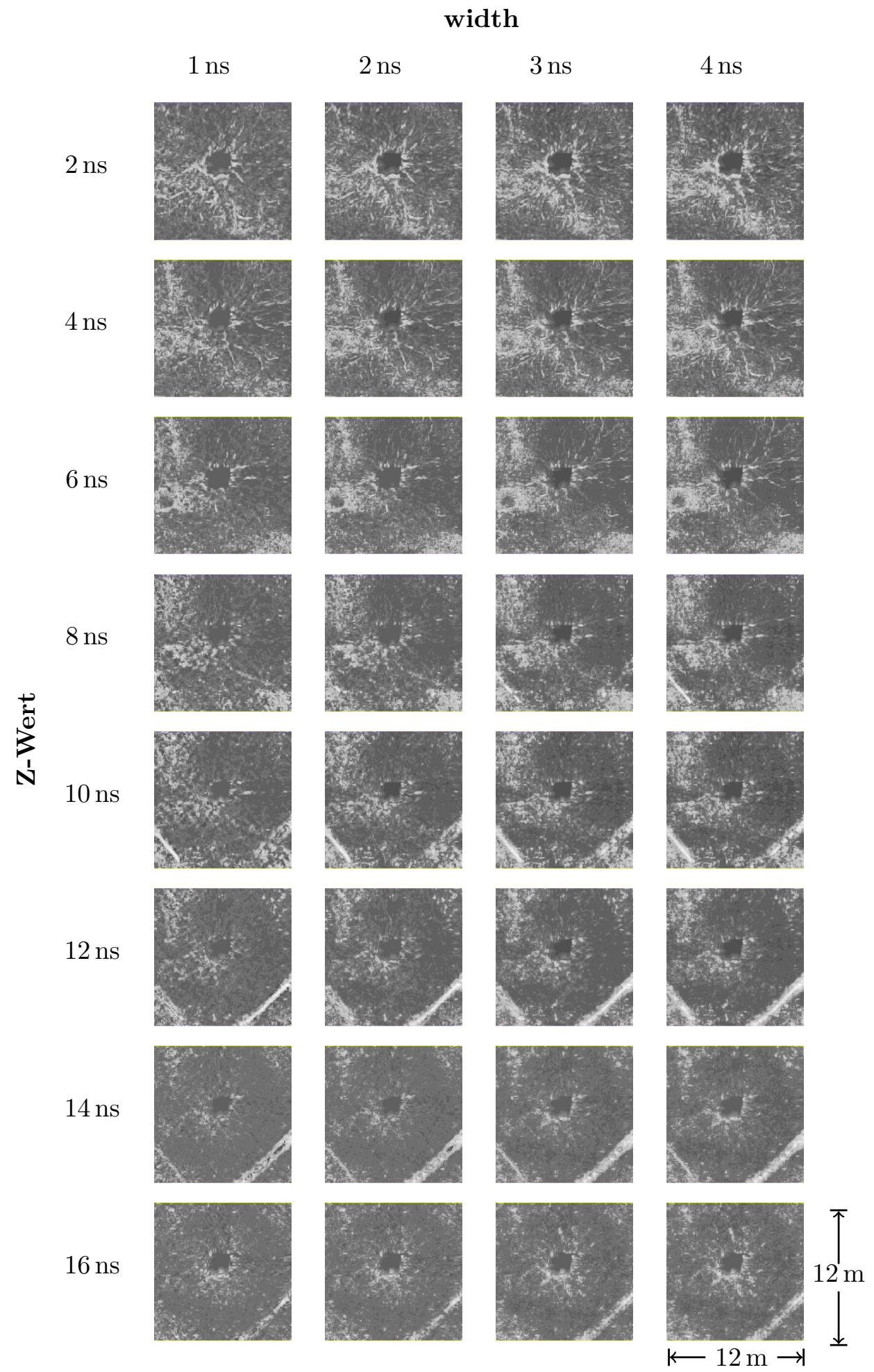

Abbildung A.15: Ergebnis der Georadaruntersuchung auf der Messfläche Fichte. 


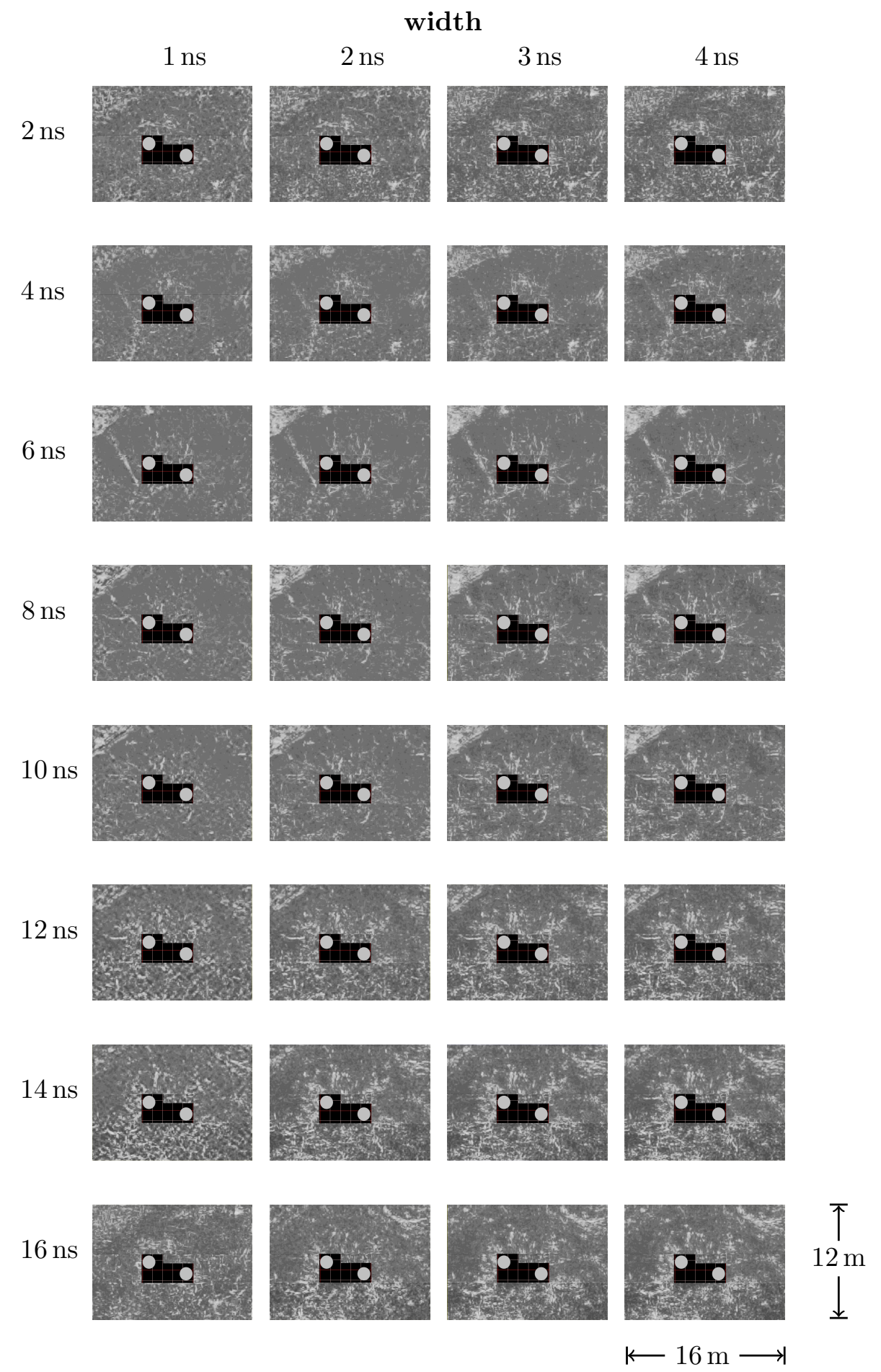

Abbildung A.16: Ergebnis der Georadaruntersuchung auf der Messfläche Buchengruppe. 

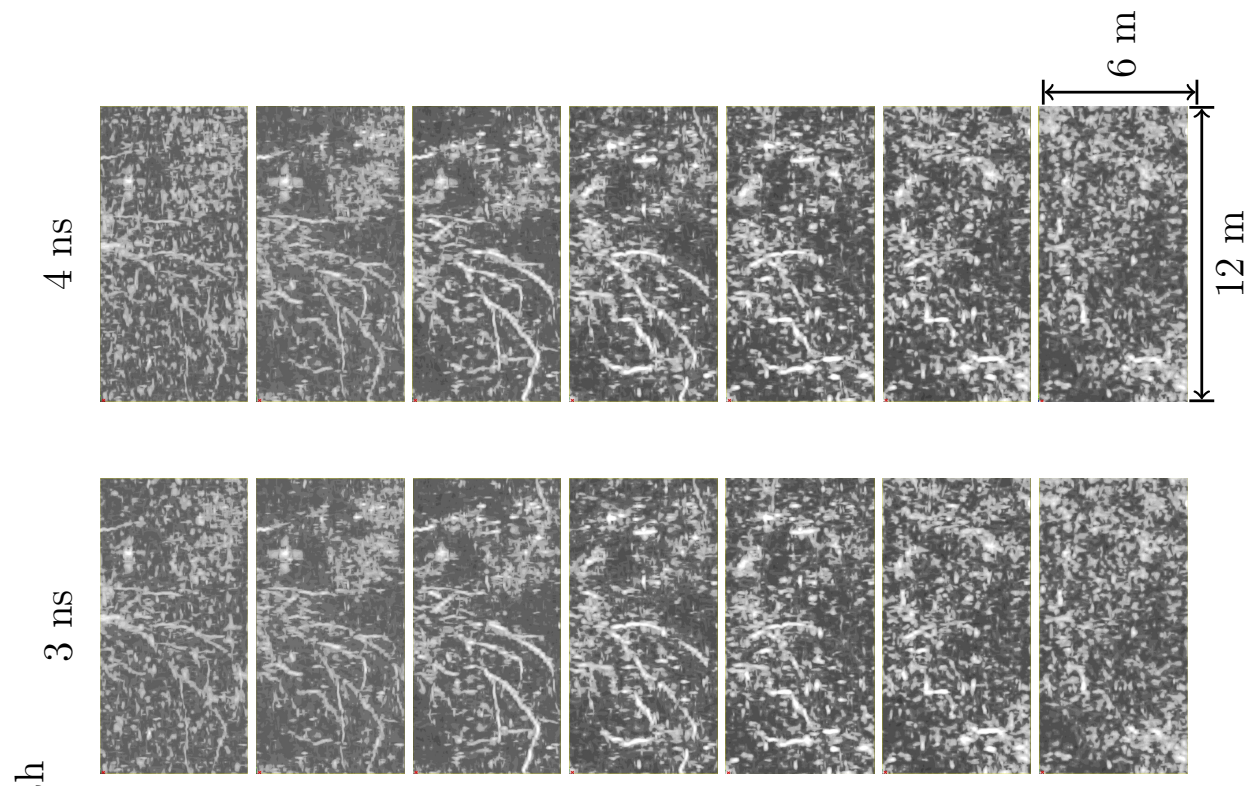

志
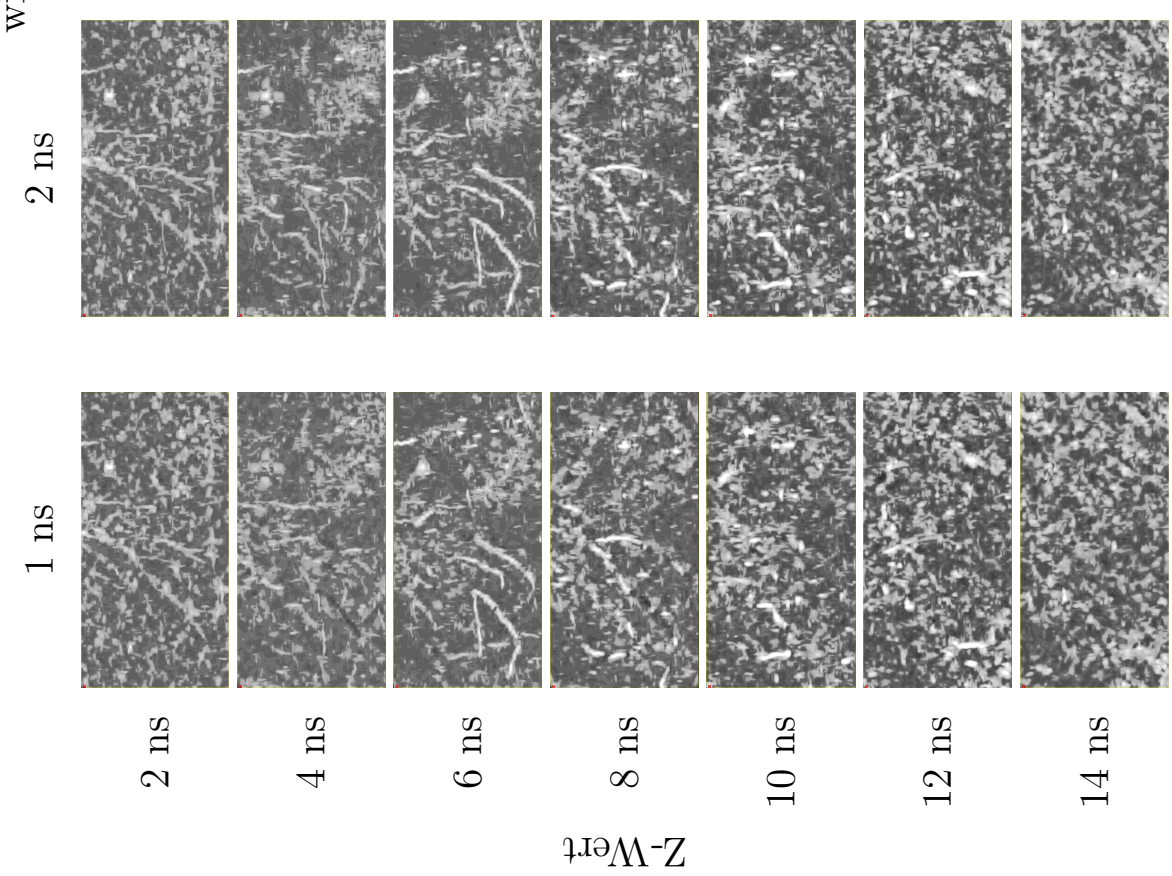

Abbildung A.17: Ergebnis der Georadaruntersuchung auf der Messfläche Platane 


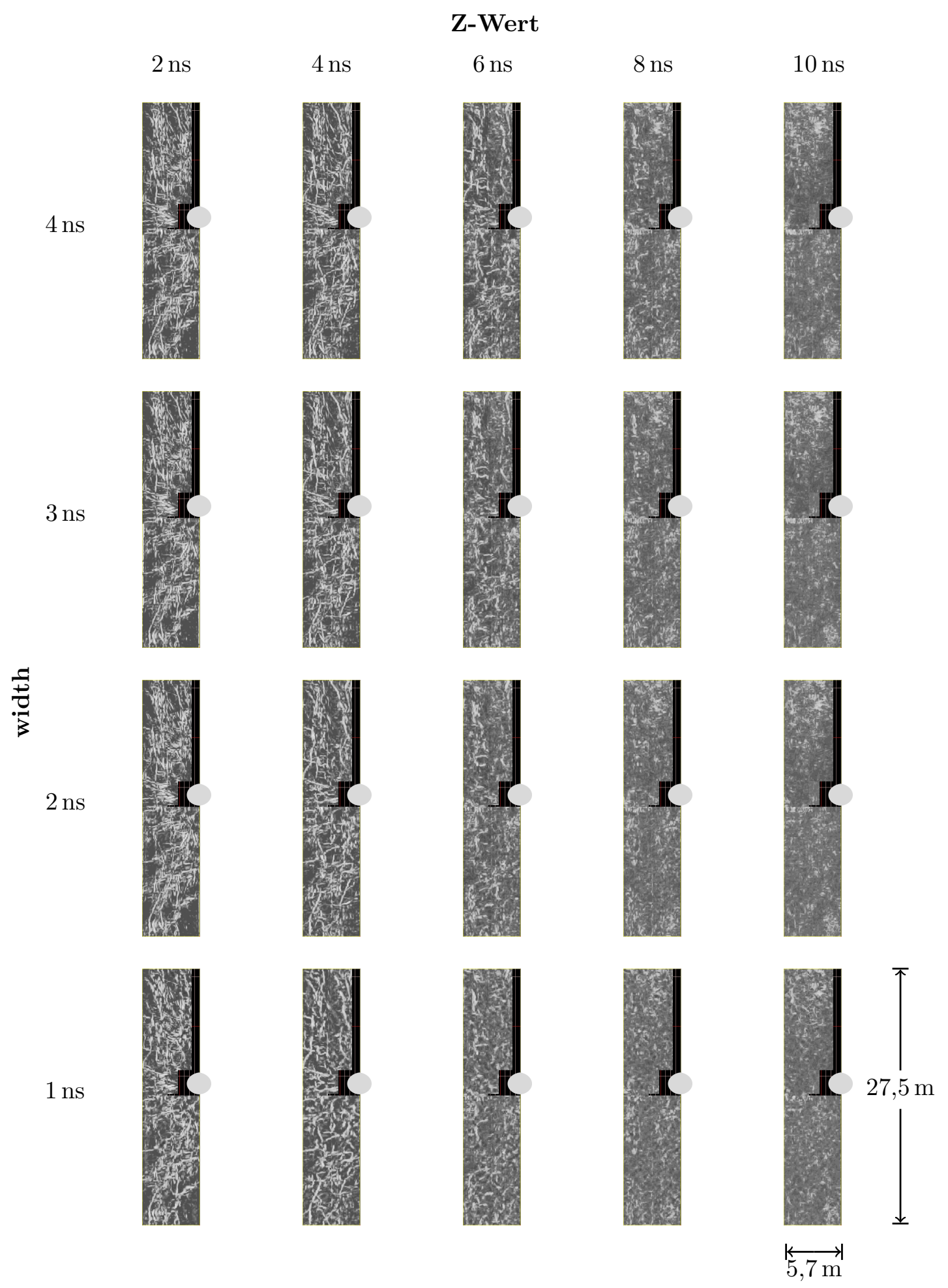

Abbildung A.18: Ergebnis der Georadaruntersuchung auf der Messfläche Silberahorn. 


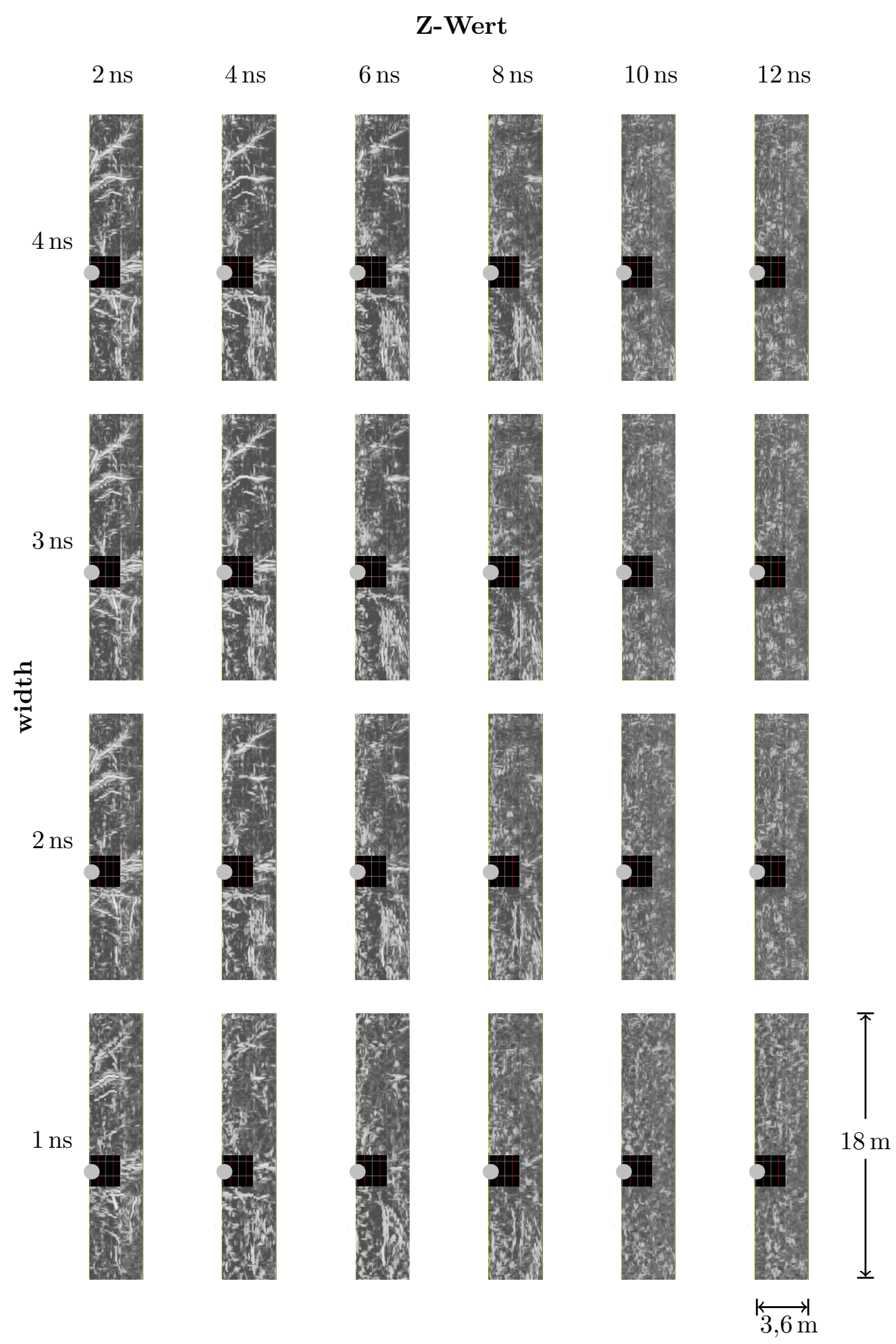

Abbildung A.19: Ergebnis der Georadaruntersuchung auf der Messfläche Hybridpappel. 


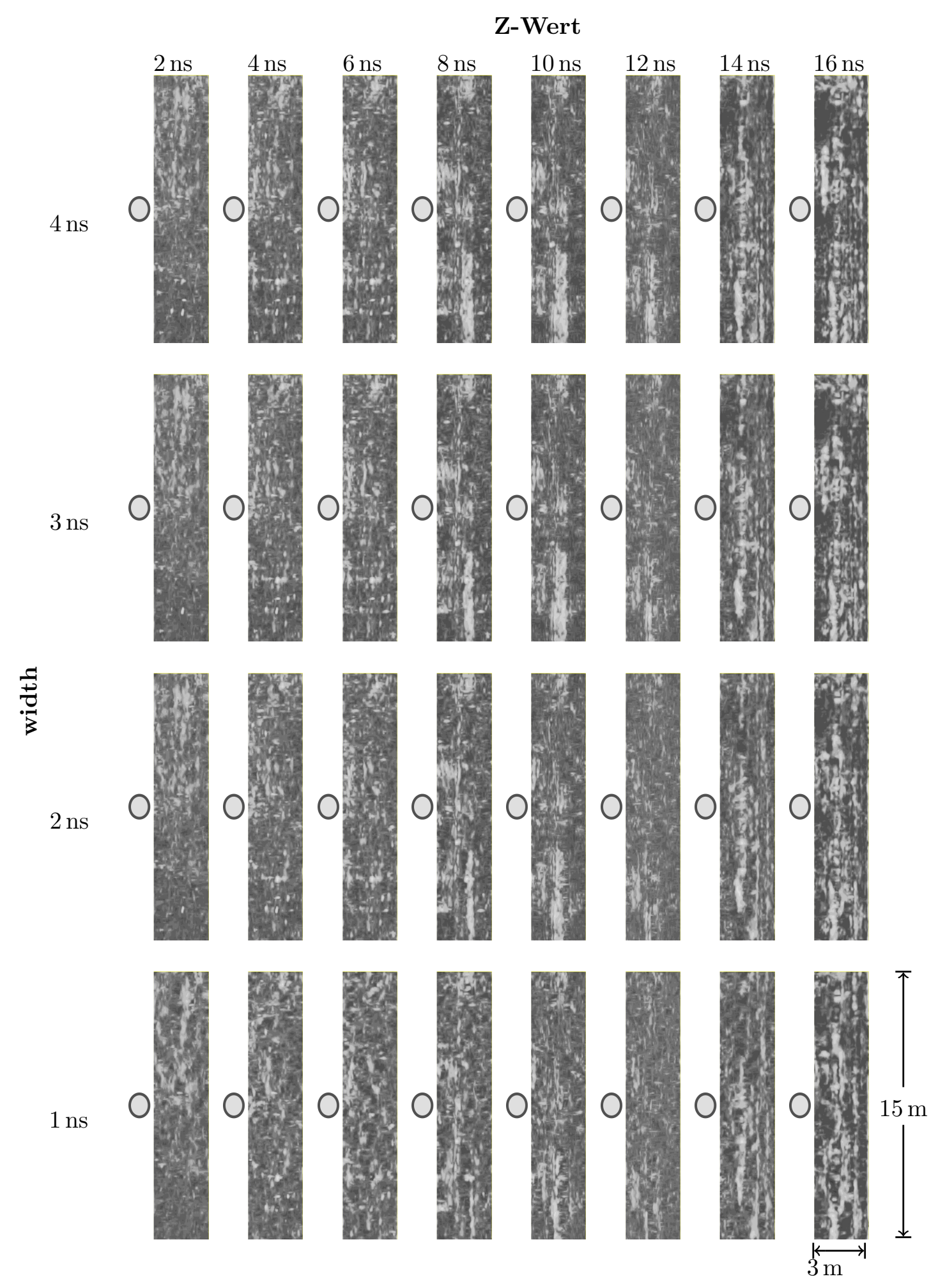

Abbildung A.20: Ergebnis der Georadaruntersuchung auf der Messfläche Große Kastanie. 


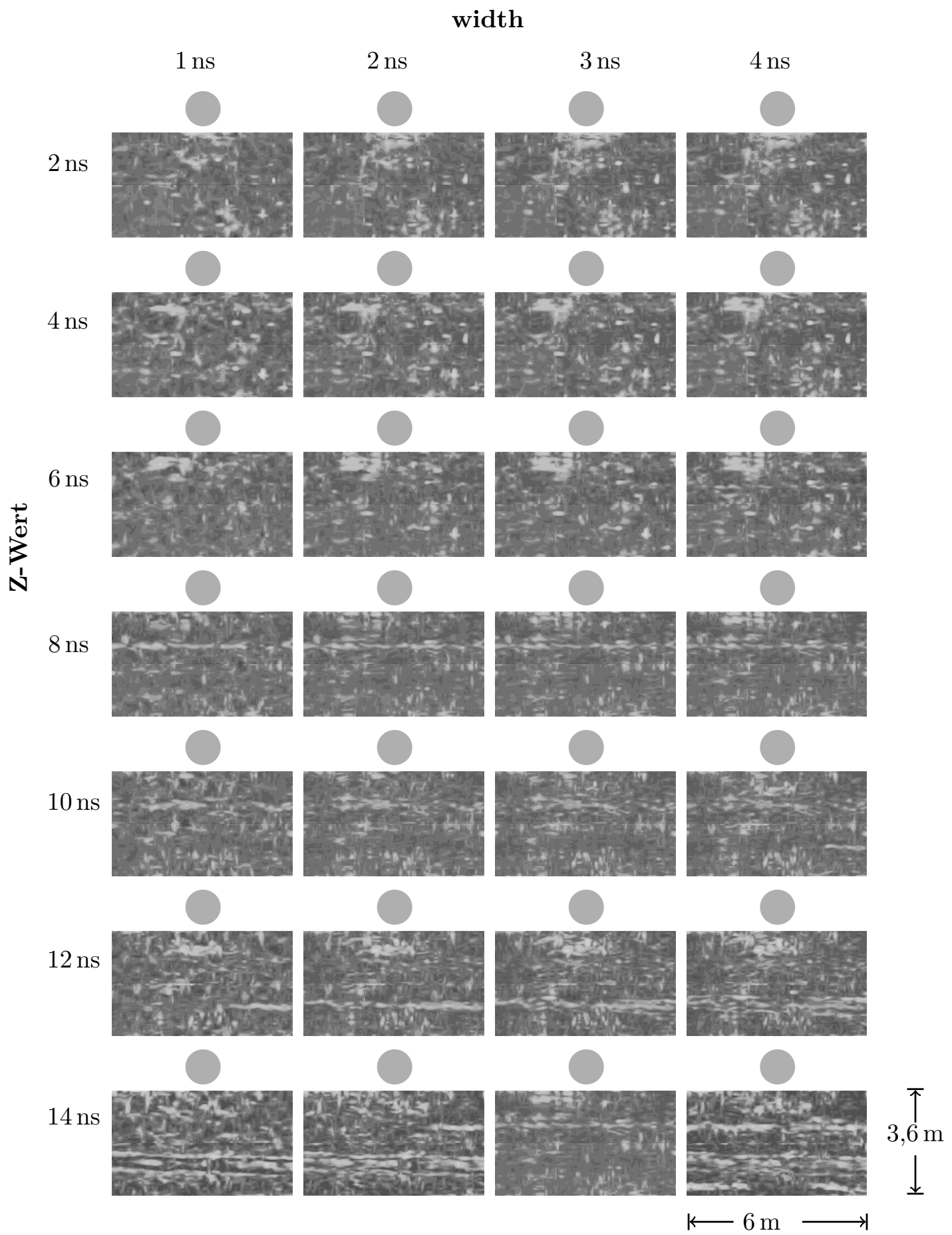

Abbildung A.21: Ergebnis der Georadaruntersuchung auf der Messfläche Kleine Kastanie. 


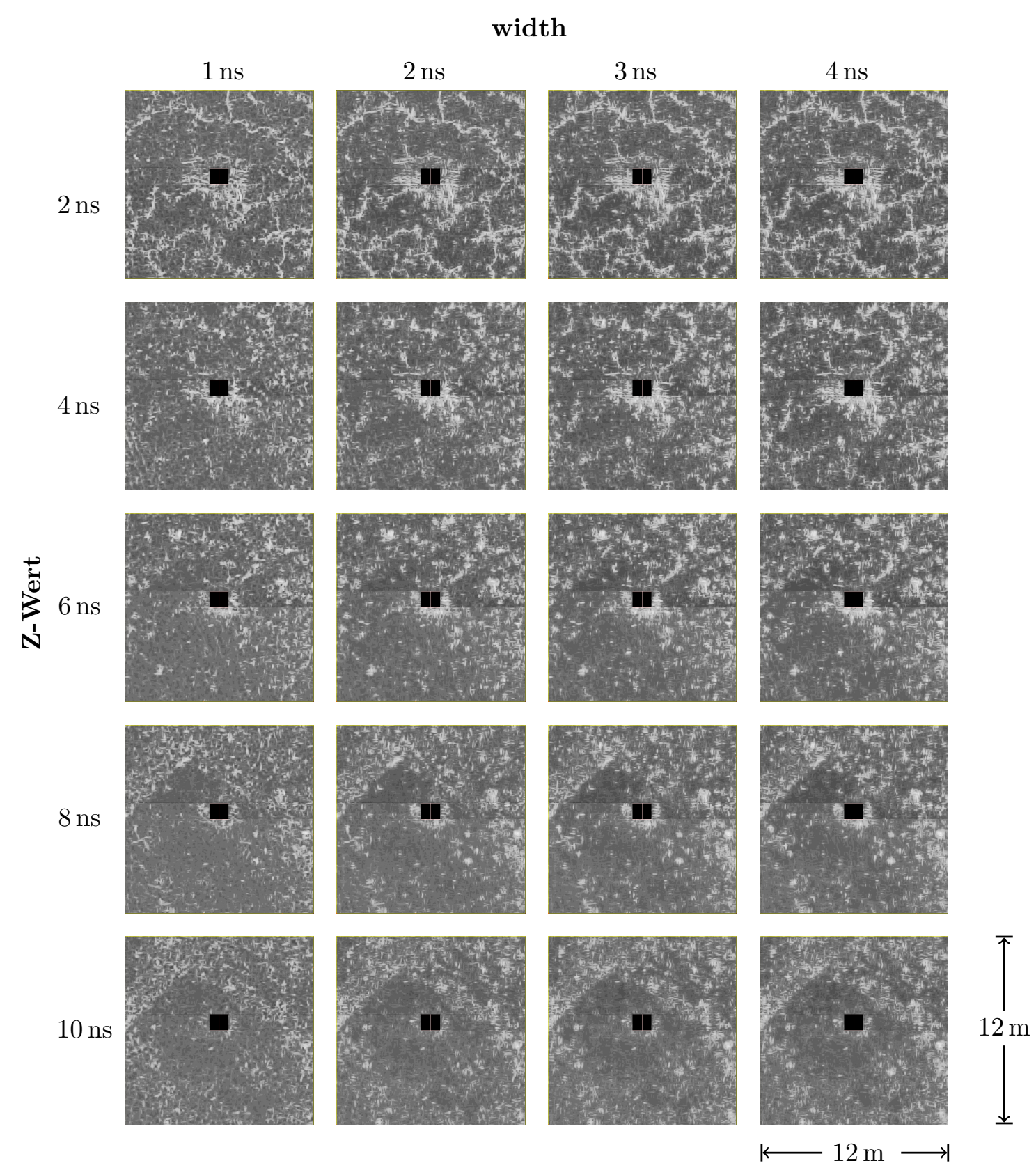

Abbildung A.22: Ergebnis der Georadaruntersuchung auf der Messfläche Amberbaum Göttingen. 

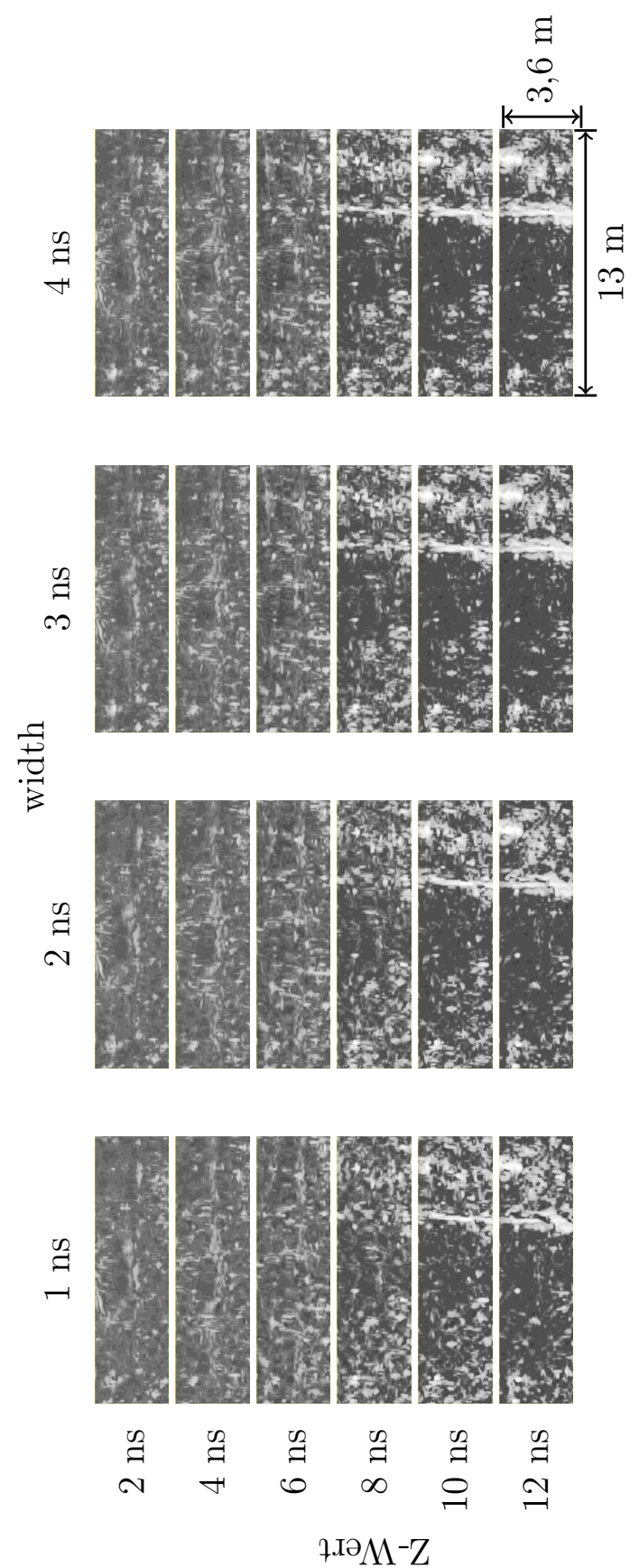

Abbildung A.23: Ergebnis der Georadaruntersuchung auf der Messfläche Kastanien Goldschmidtstraße (Baum 1). 

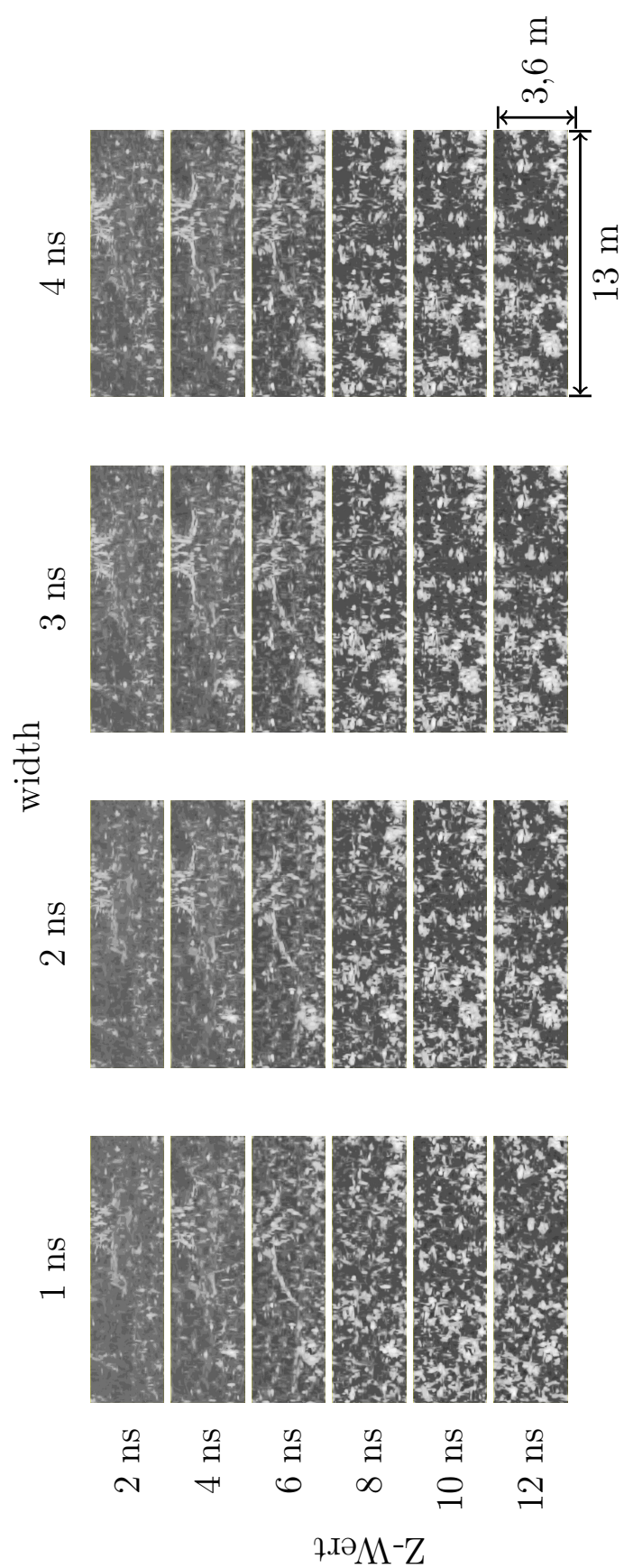

Abbildung A.24: Ergebnis der Georadaruntersuchung auf der Messfläche Kastanien Goldschmidtstraße (Baum 2). 

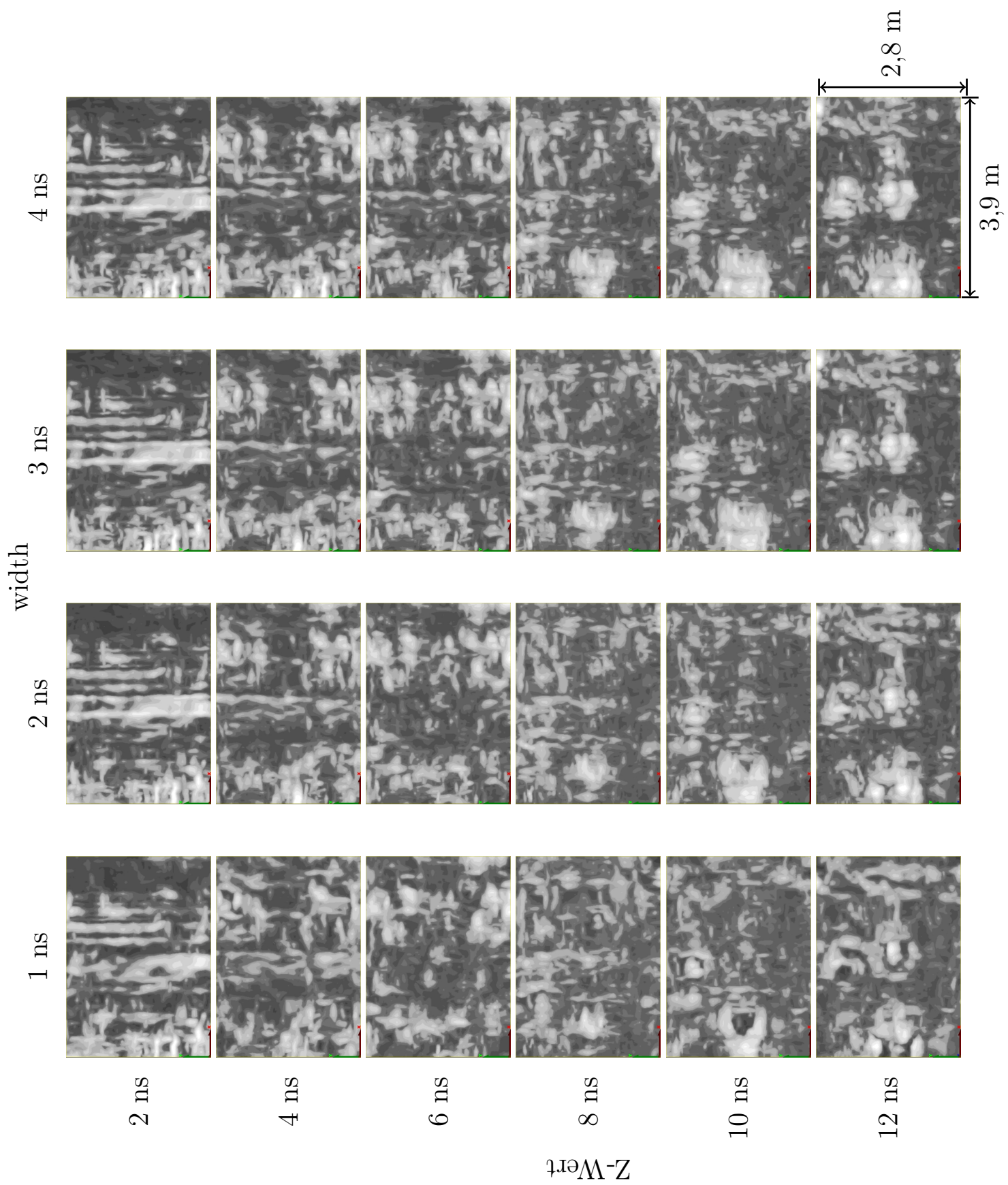

Abbildung A.25: Ergebnis der Georadaruntersuchung auf der Messfläche Kastanie Nimwegen. 

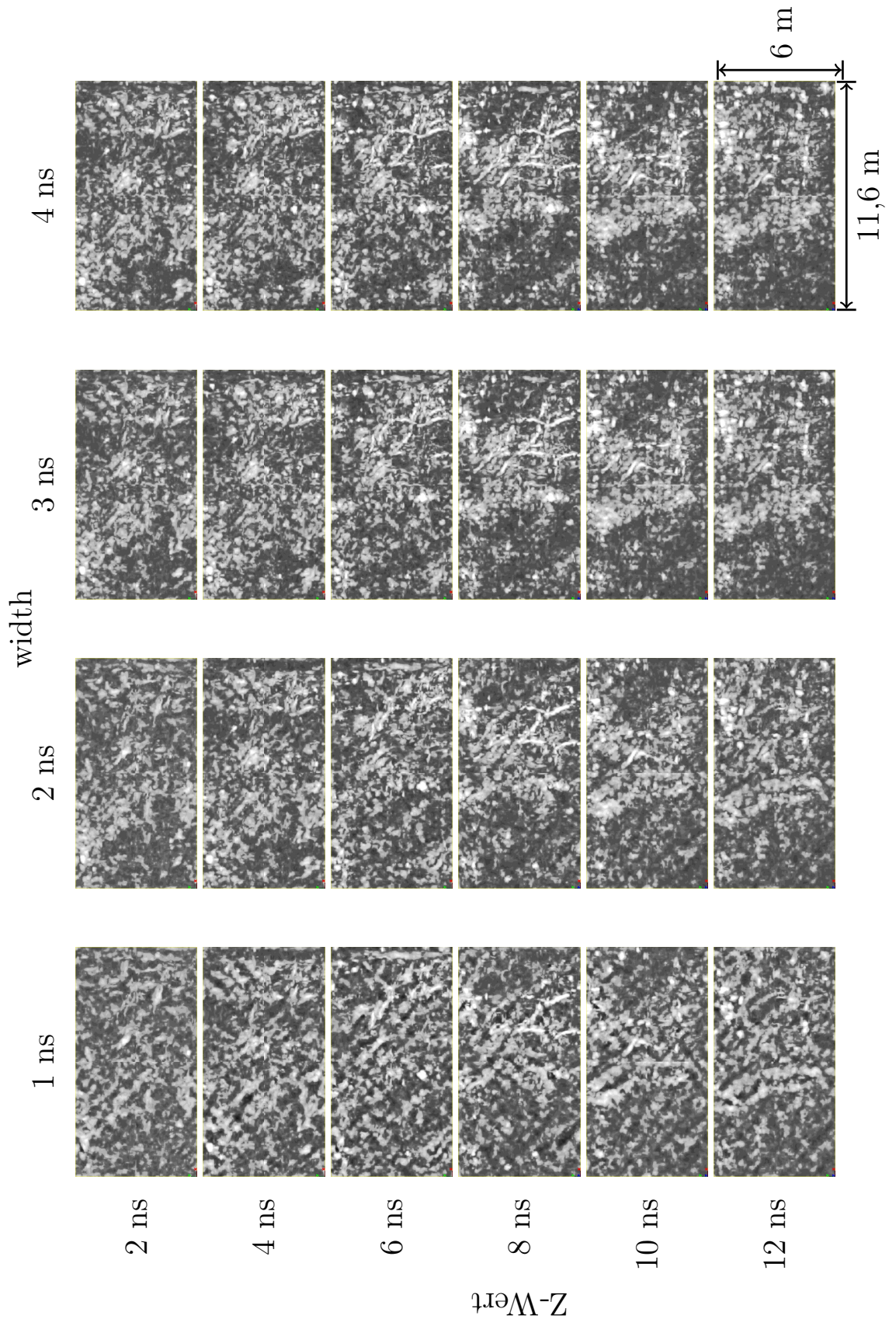

Abbildung A.26: Ergebnis der Georadaruntersuchung auf der Messfläche Kastanie Fulda. 
A.4 Gesamtradargramme und -tomogramme der einzelnen Messflächen
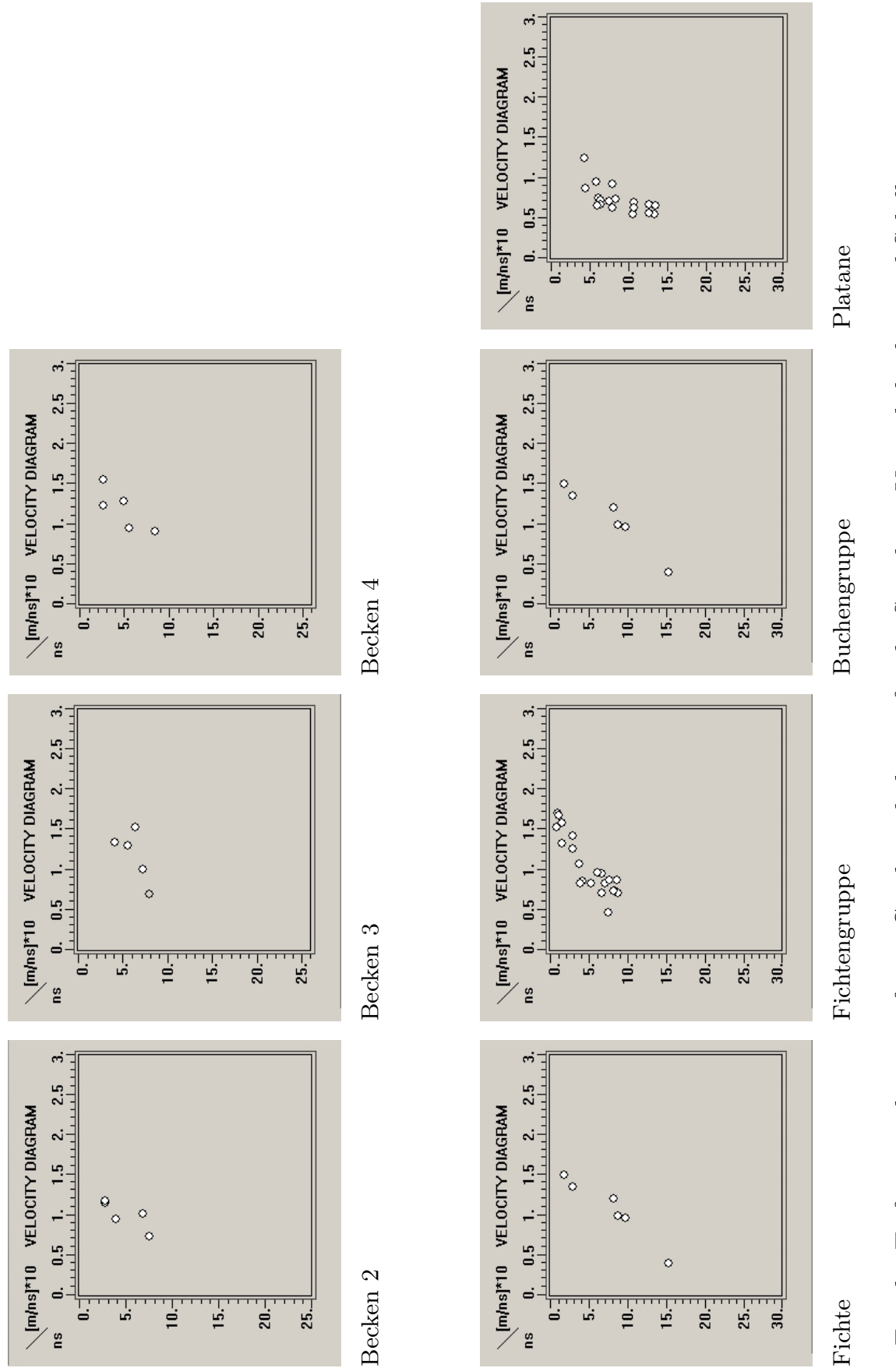

$\vec{n}$

0
0
0
0
0
0
0
0
0
0
0
0
0
0
0
0
0
0
0
0
0
0

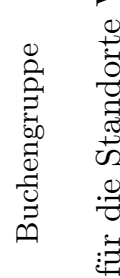

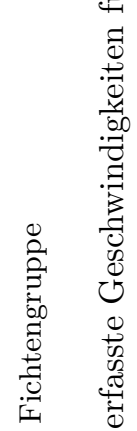
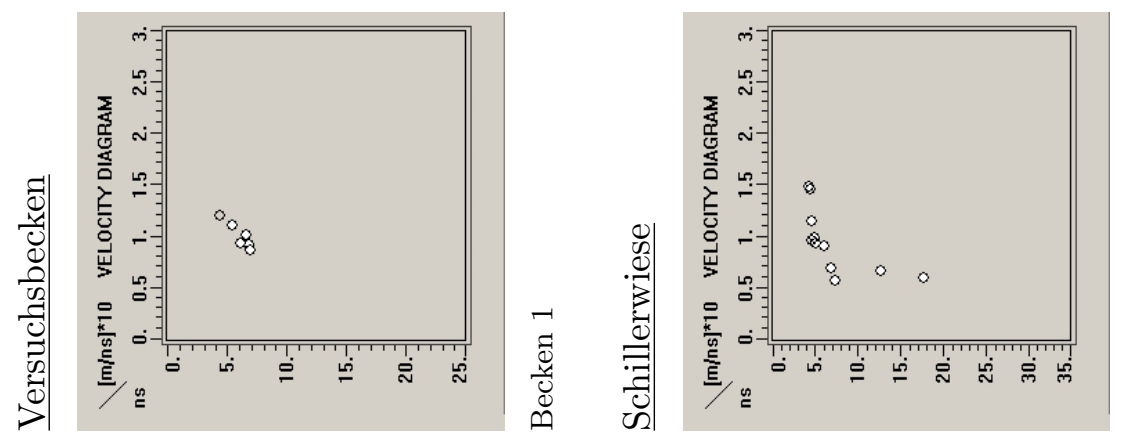

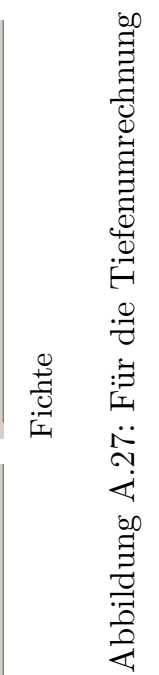


A Anhang
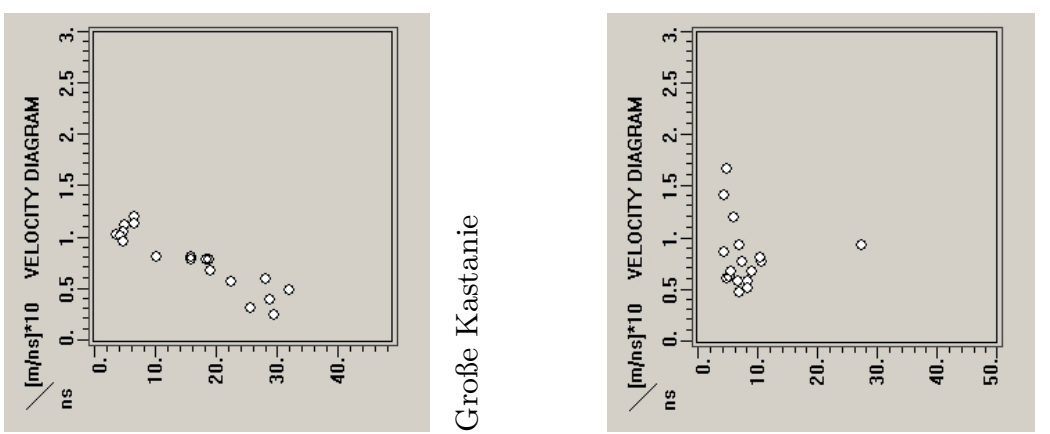

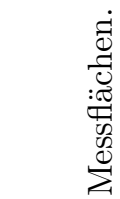
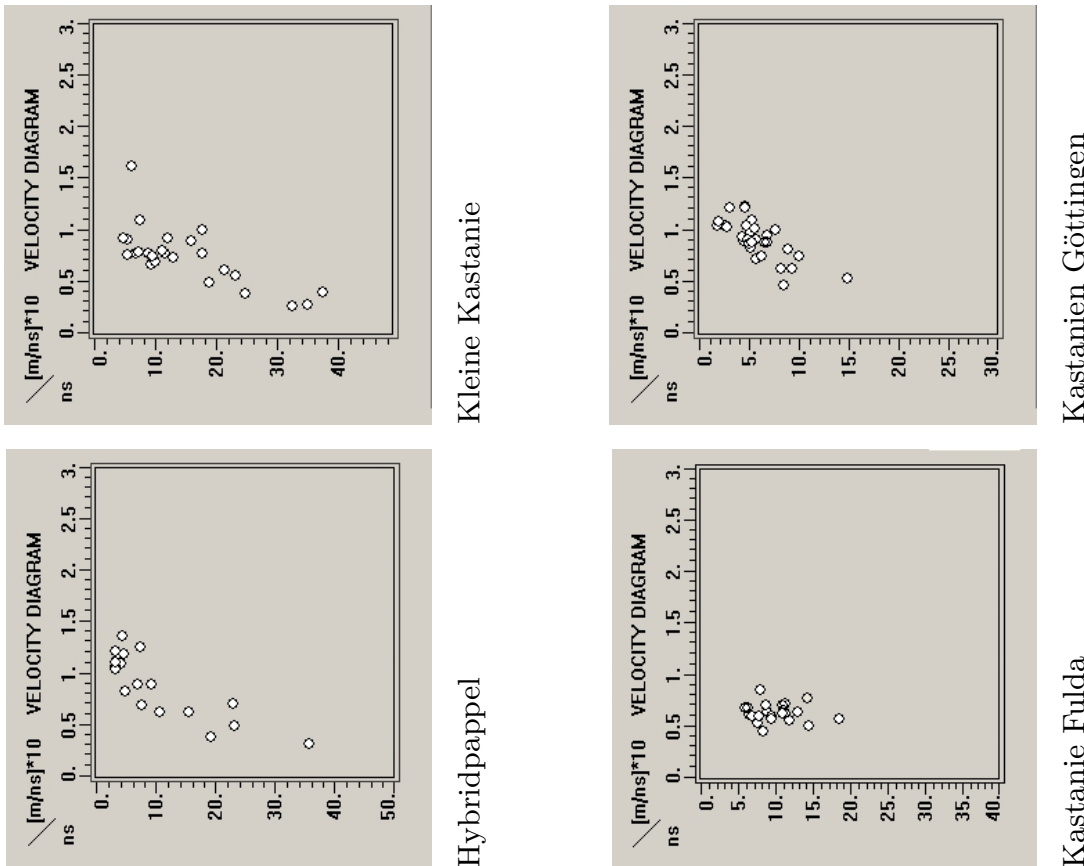

进
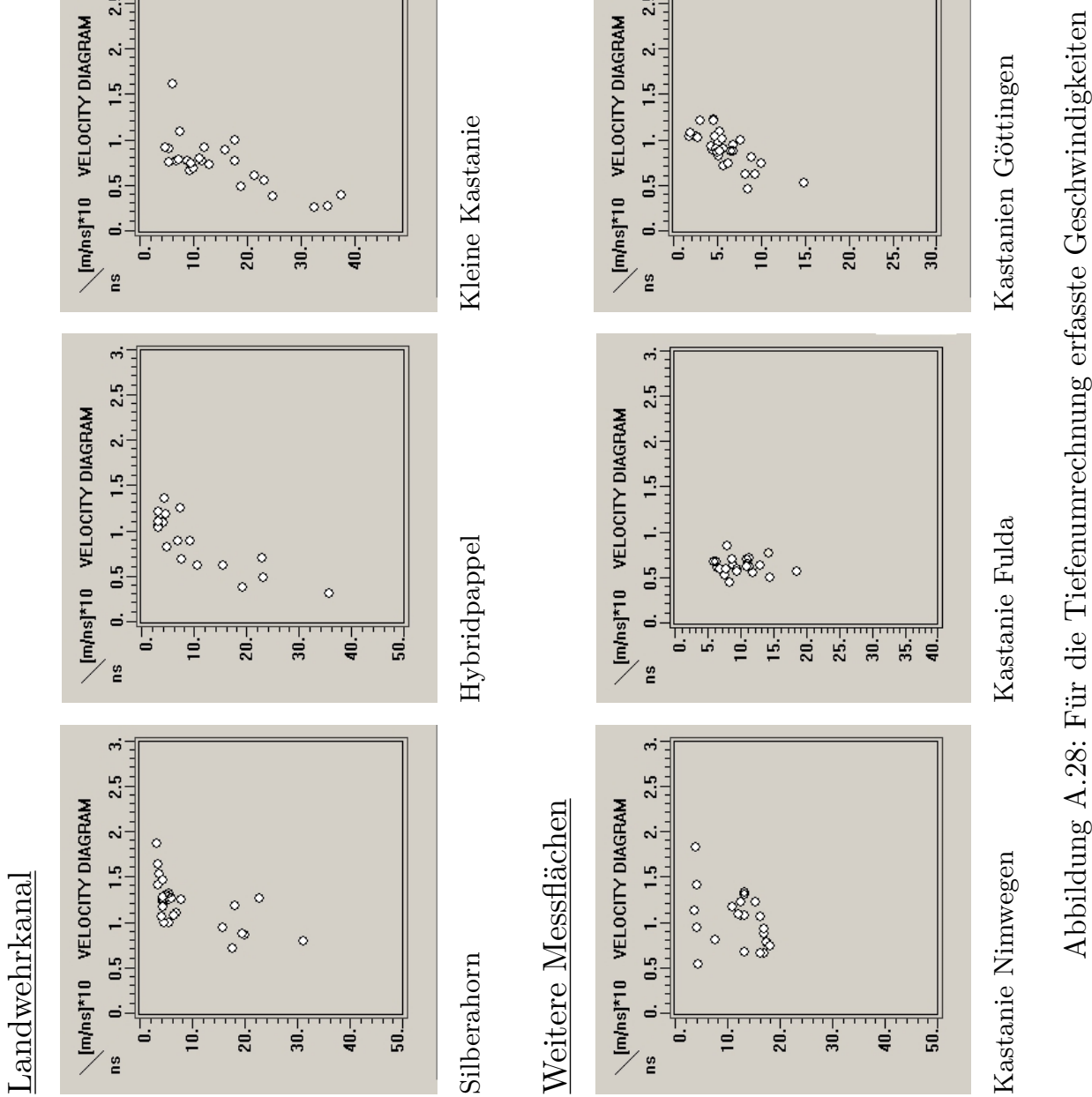

: 


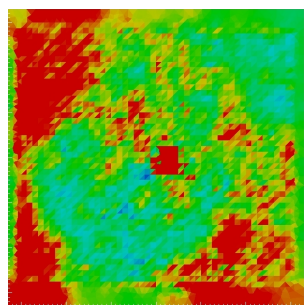

$0 \mathrm{~m}$

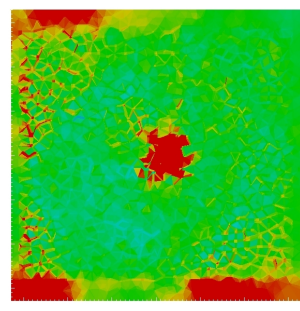

$0.4 \mathrm{~m}$

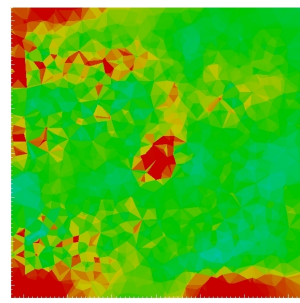

$0.8 \mathrm{~m}$

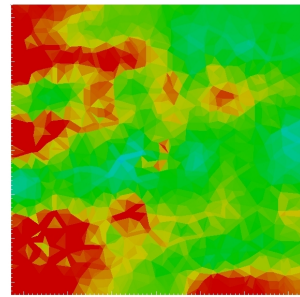

$1.2 \mathrm{~m}$

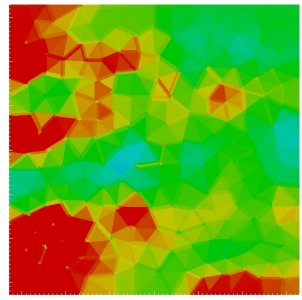

$1.7 \mathrm{~m}$

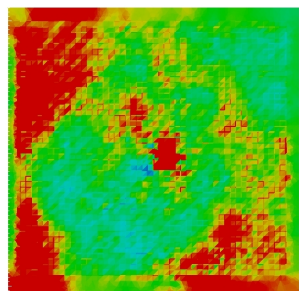

$0.1 \mathrm{~m}$

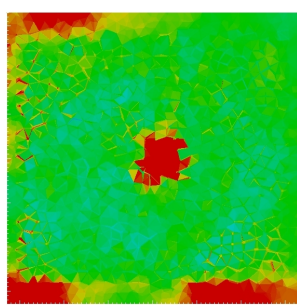

$0.5 \mathrm{~m}$

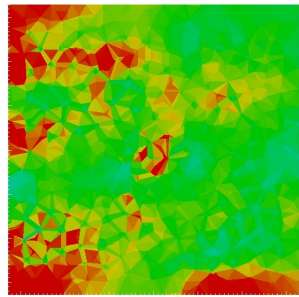

$0.9 \mathrm{~m}$

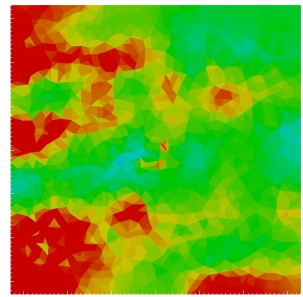

$1.3 \mathrm{~m}$

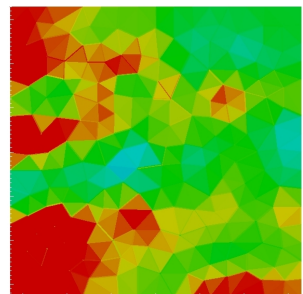

$1.8 \mathrm{~m}$

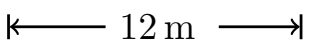

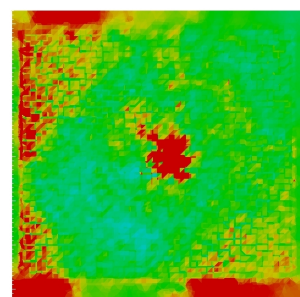

$0.2 \mathrm{~m}$

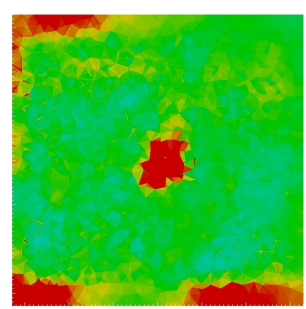

$0.6 \mathrm{~m}$

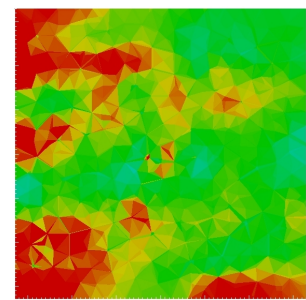

$1.0 \mathrm{~m}$

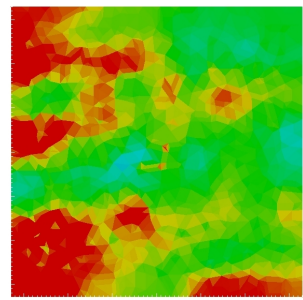

$1.4 \mathrm{~m}$

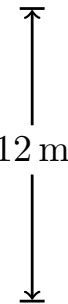

$12 \mathrm{~m}$

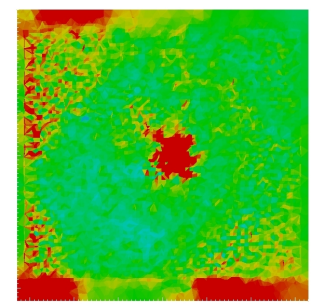

$0.3 \mathrm{~m}$

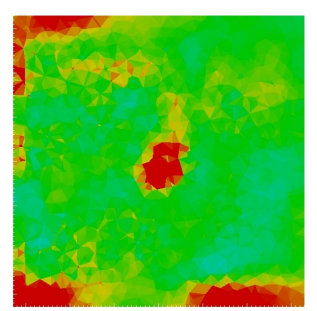

$0.7 \mathrm{~m}$

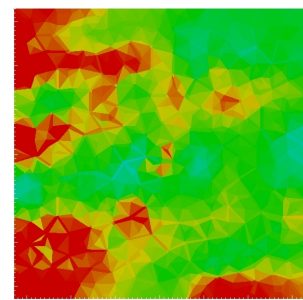

$1.1 \mathrm{~m}$

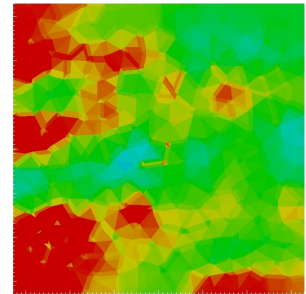

$1.6 \mathrm{~m}$

Abbildung A.29: Ergebnis der Geoelektrikuntersuchung auf der Messfläche Schwarzkiefer. 

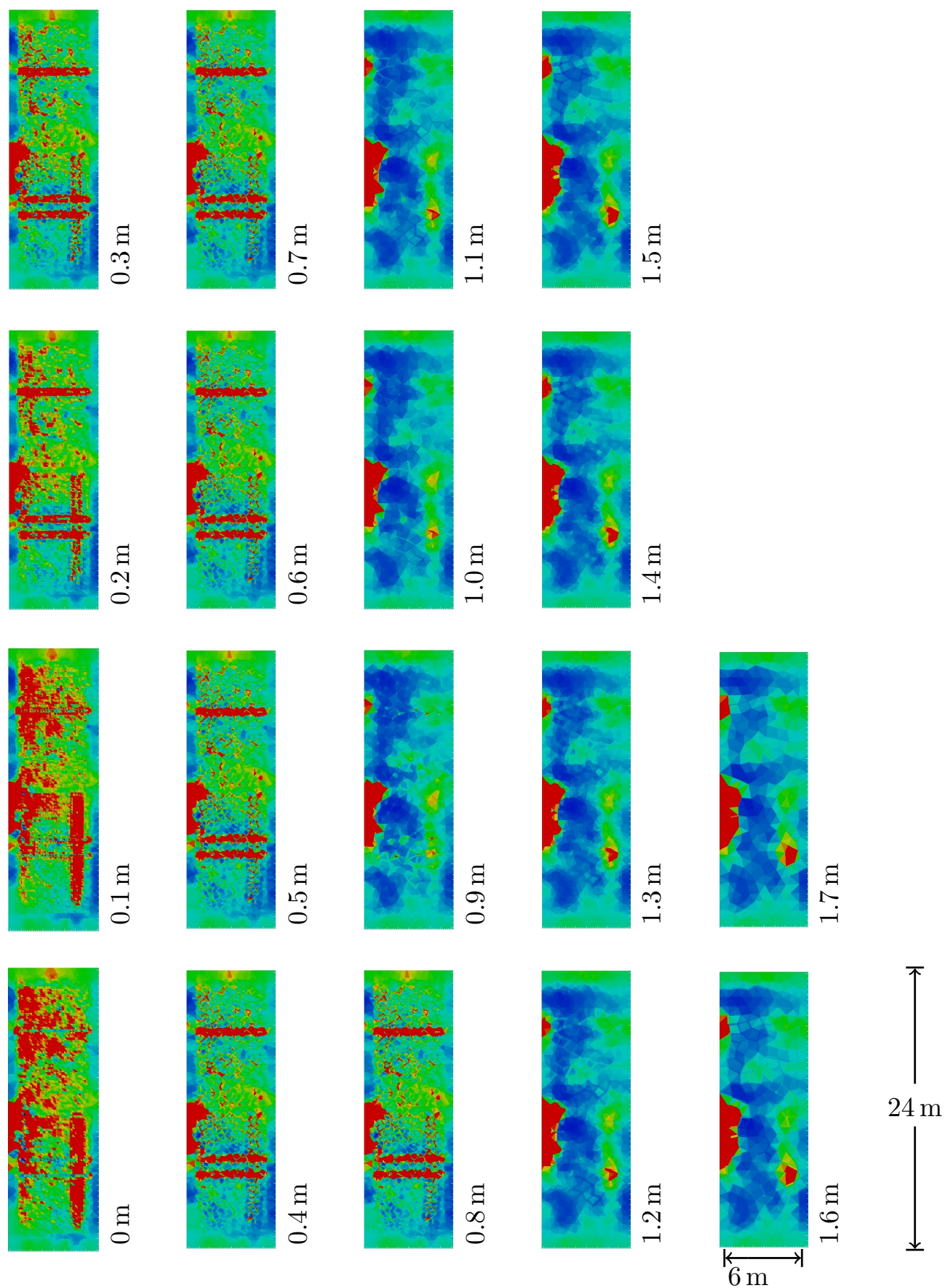

Abbildung A.30: Ergebnis der Geoelektrikuntersuchung auf der Messfläche Fichtengruppe. 

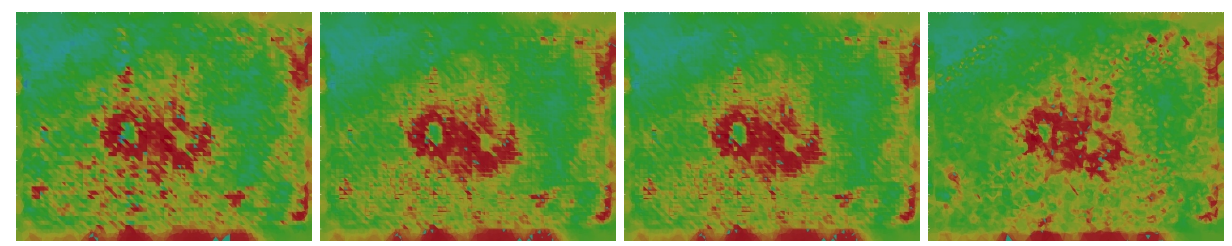

$0 \mathrm{~m}$

$0.1 \mathrm{~m}$

$0.2 \mathrm{~m}$

$0.3 \mathrm{~m}$
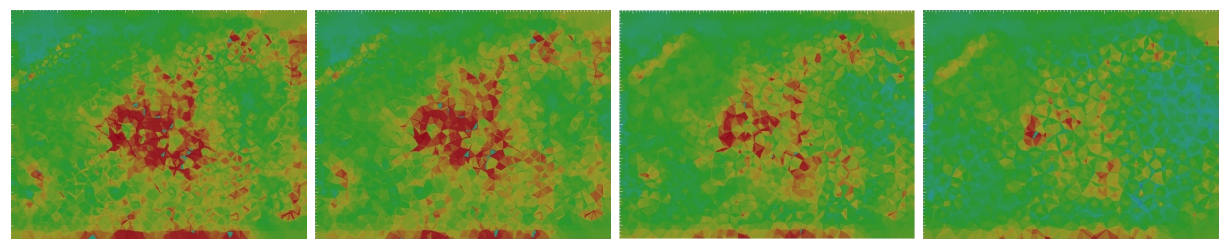

$0.4 \mathrm{~m}$

$0.5 \mathrm{~m}$

$0.6 \mathrm{~m}$

$0.7 \mathrm{~m}$
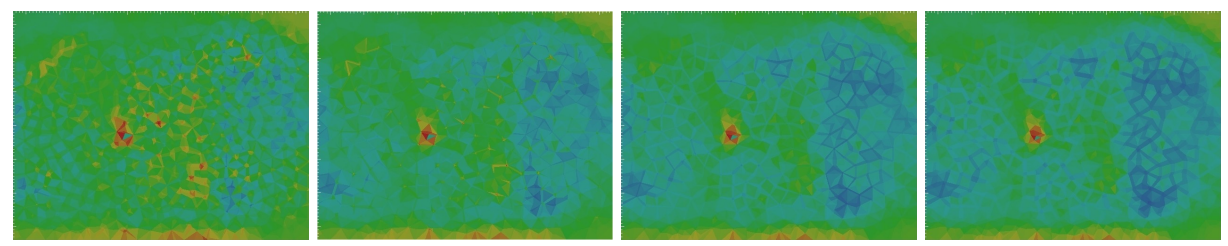

$0.8 \mathrm{~m}$

$0.9 \mathrm{~m}$

$1.0 \mathrm{~m}$

$1.1 \mathrm{~m}$
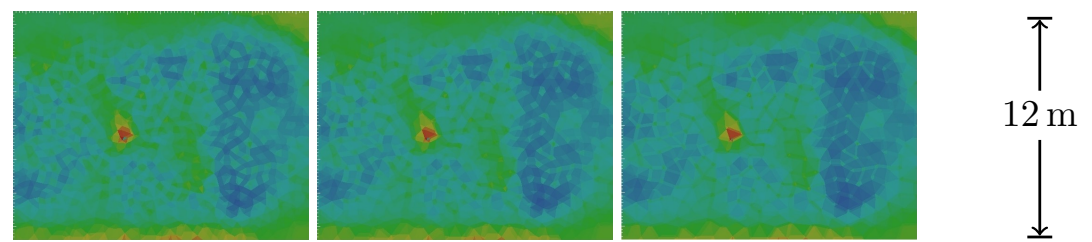

$1.2 \mathrm{~m}$

$1.3 \mathrm{~m}$

$1.4 \mathrm{~m}$

Abbildung A.31: Ergebnis der Geoelektrikuntersuchung auf der Messfläche Buchengruppe. 

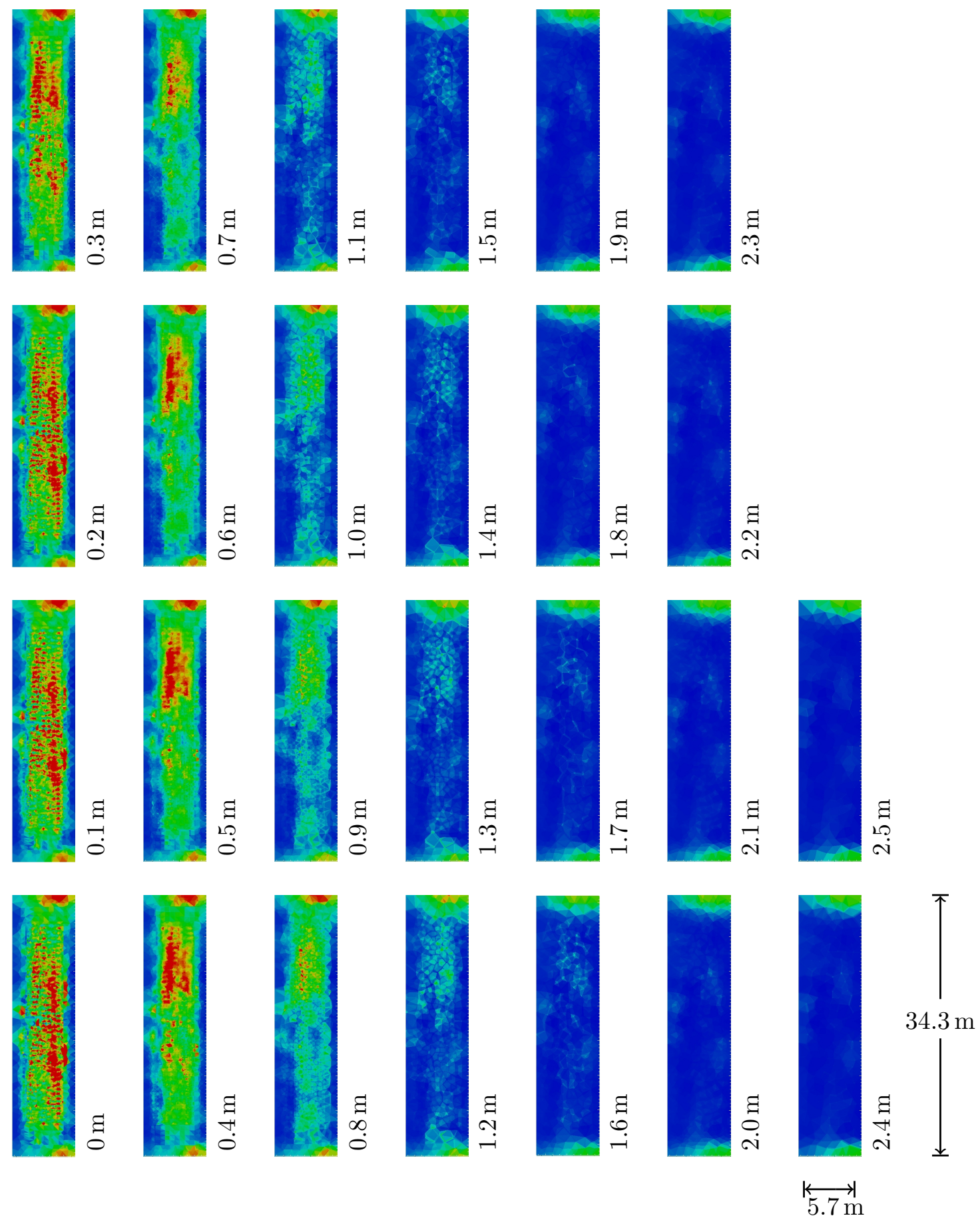

Abbildung A.32: Ergebnis der Geoelektrikuntersuchung auf der Messfläche Silberahorn. 

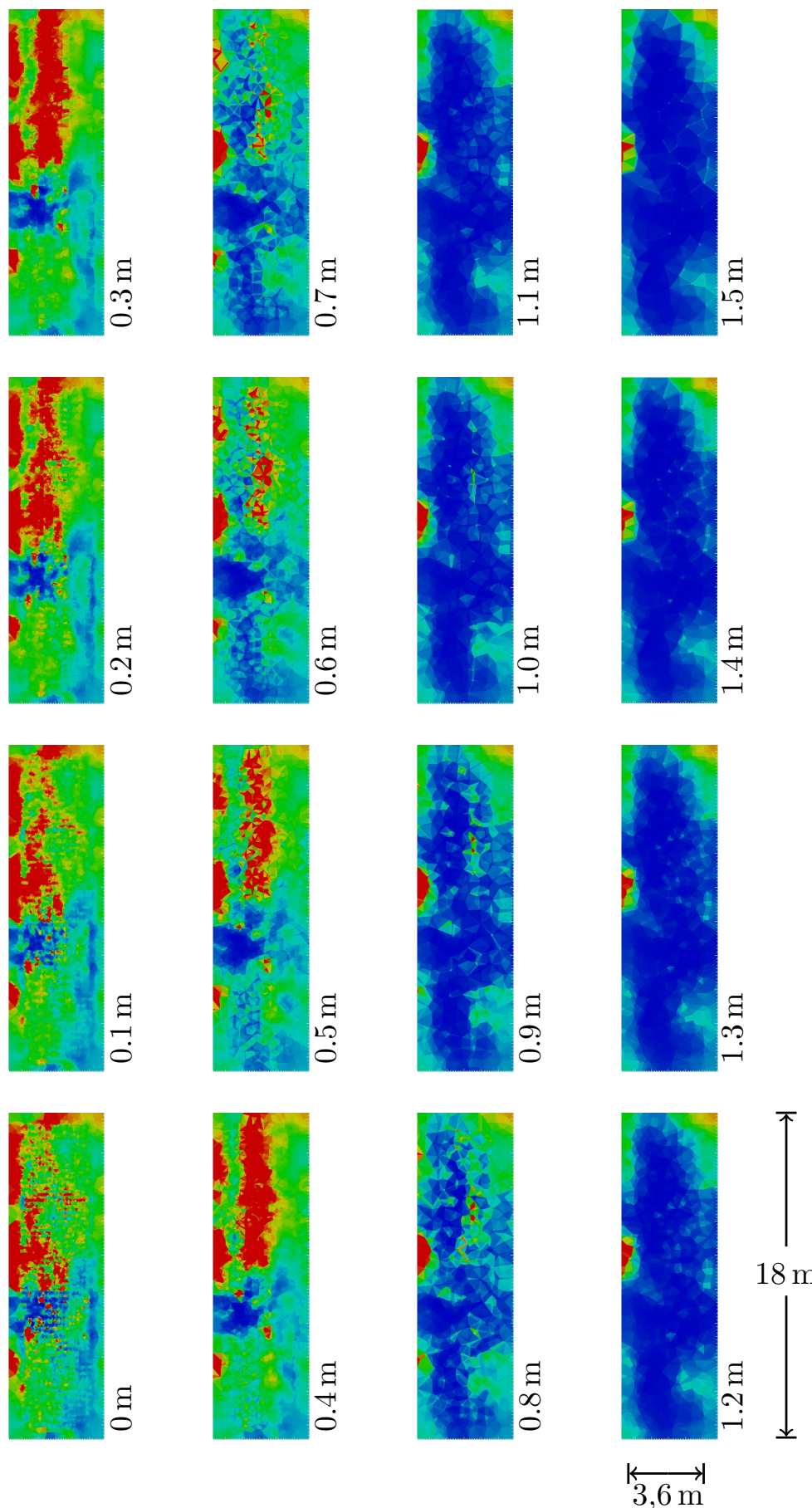

Abbildung A.33: Ergebnis der Geoelektrikuntersuchung auf der Messfläche Hybridpappel. 

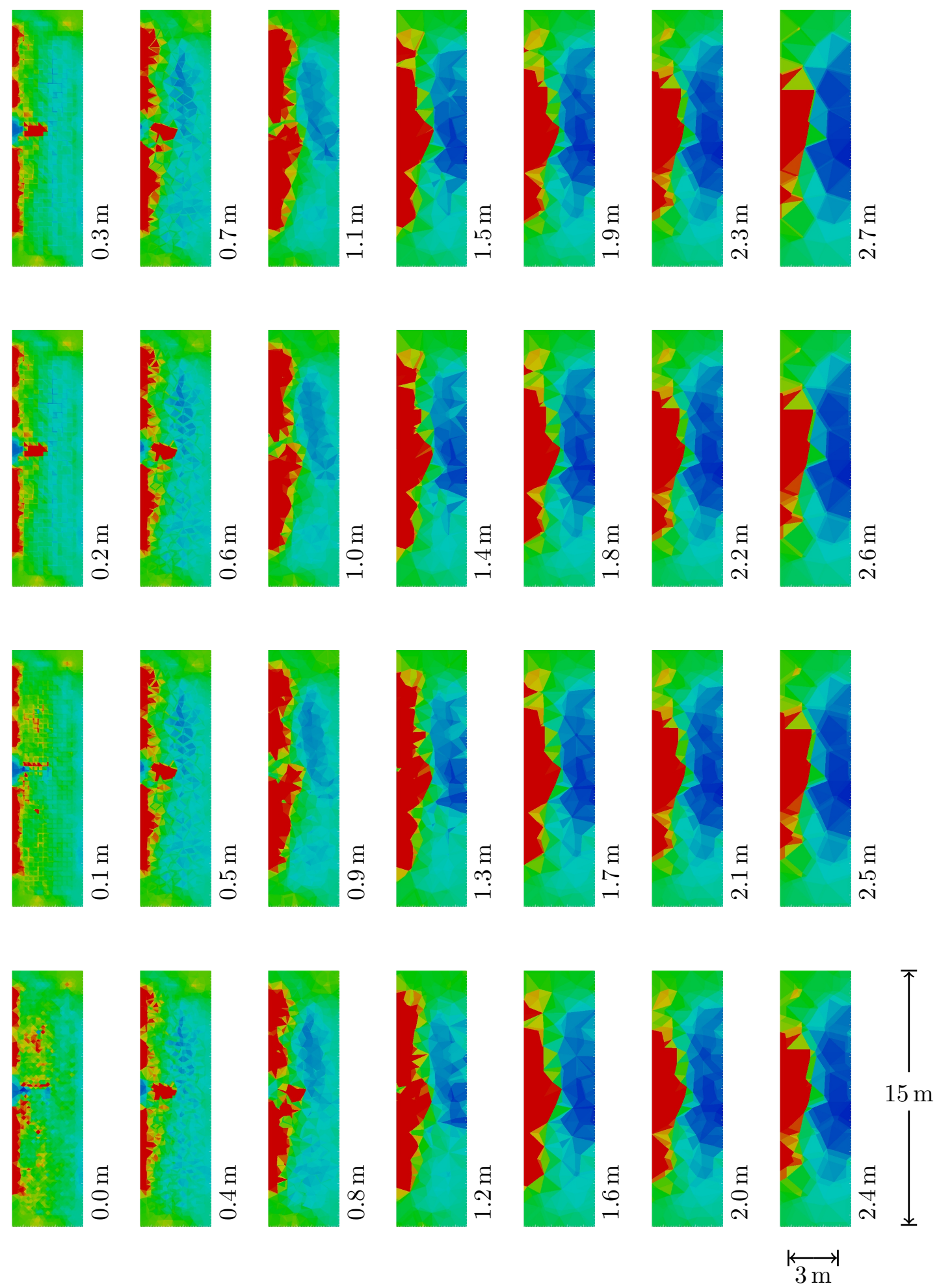

Abbildung A.34: Ergebnis der Geoelektrikuntersuchung auf der Messfläche Große Kastanie. 

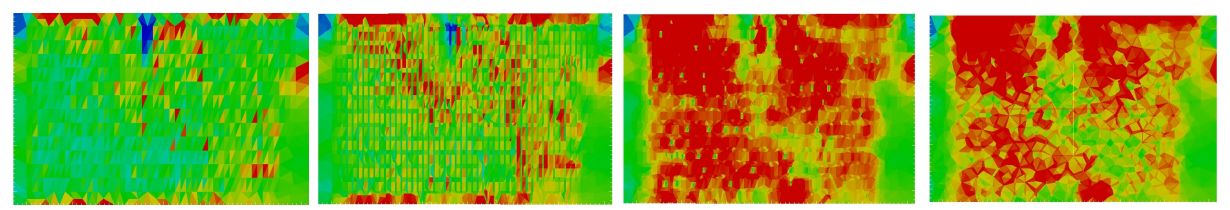

$0 \mathrm{~m}$

$0.1 \mathrm{~m}$

$0.2 \mathrm{~m}$

$0.3 \mathrm{~m}$
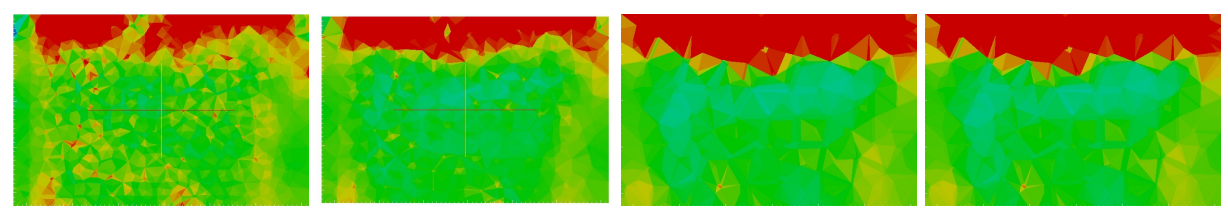

$0.4 \mathrm{~m}$

$0.5 \mathrm{~m}$

$0.6 \mathrm{~m}$

$0.7 \mathrm{~m}$
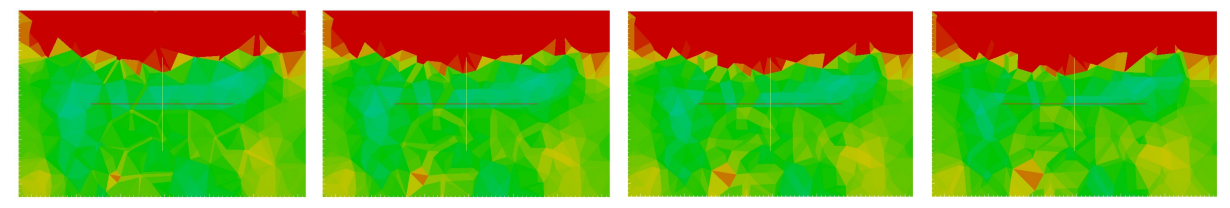

$0.8 \mathrm{~m}$

$0.9 \mathrm{~m}$

$1.0 \mathrm{~m}$

$1.1 \mathrm{~m}$
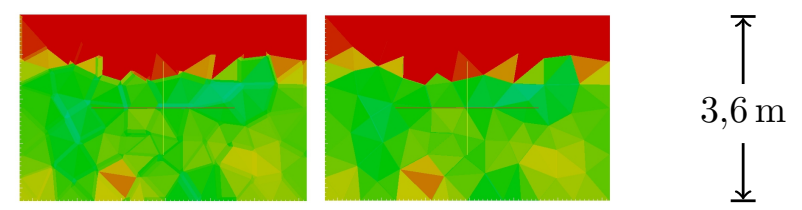

$1.2 \mathrm{~m}$

$1.3 \mathrm{~m}$

$\longleftarrow 6 \mathrm{~m} \longrightarrow$

Abbildung A.35: Ergebnis der Geoelektrikuntersuchung auf der Messfläche Kleine Kastanie. 